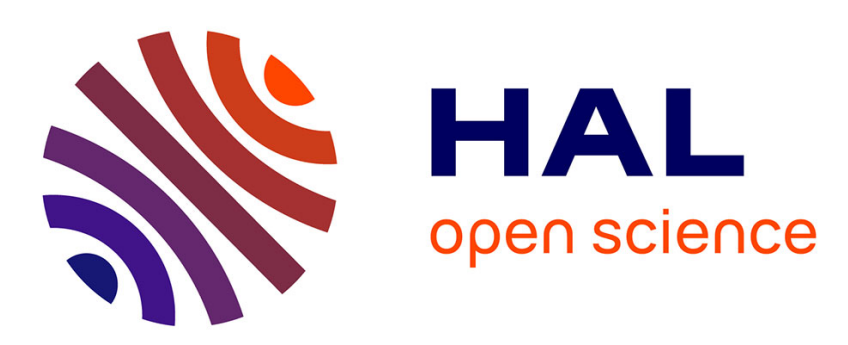

\title{
Indigo, outil multimédia d'éducation thérapeutique destiné à des patients diabétiques de type 1 adultes : évaluation du point de vue des patients utilisateurs Carole Knapp
}

\section{- To cite this version:}

Carole Knapp. Indigo, outil multimédia d'éducation thérapeutique destiné à des patients diabétiques de type 1 adultes : évaluation du point de vue des patients utilisateurs. Médecine humaine et pathologie. 2013. dumas-00921467

\section{HAL Id: dumas-00921467 https://dumas.ccsd.cnrs.fr/dumas-00921467}

Submitted on 20 Dec 2013

HAL is a multi-disciplinary open access archive for the deposit and dissemination of scientific research documents, whether they are published or not. The documents may come from teaching and research institutions in France or abroad, or from public or private research centers.
L'archive ouverte pluridisciplinaire HAL, est destinée au dépôt et à la diffusion de documents scientifiques de niveau recherche, publiés ou non, émanant des établissements d'enseignement et de recherche français ou étrangers, des laboratoires publics ou privés. 


\section{Université
Joseph Fourier MEDECINE}

\section{SICD1 \\ Bibliothèques \\ de I'UJF et de Grenoble INP}

\section{AVERTISSEMENT}

Ce document est le fruit d'un long travail approuvé par le jury de soutenance et mis à disposition de l'ensemble de la communauté universitaire élargie.

Il n'a pas été réévalué depuis la date de soutenance.

Il est soumis à la propriété intellectuelle de l'auteur. Ceci implique une obligation de citation et de référencement lors de l'utilisation de ce document.

D'autre part, toute contrefaçon, plagiat, reproduction illicite encourt une poursuite pénale.

Contact au SICD1 de Grenoble : thesebum@ujf-grenoble.fr

\section{LIENS}

Code de la Propriété Intellectuelle. articles L 122. 4

Code de la Propriété Intellectuelle. articles L 335.2- L 335.10

http://www.cfcopies.com/V2/leg/leg_droi.php

http://www.culture.gouv.fr/culture/infos-pratiques/droits/protection.htm 
UNIVERSITE JOSEPH FOURIER

FACULTE DE MEDECINE DE GRENOBLE

Année : 2013

$\mathrm{N}^{\circ}$

INDIGO, OUTIL MULTIMEDIA D'EDUCATION THERAPEUTIQUE DESTINE

A DES PATIENTS DIABETIQUES DE TYPE 1 ADULTES : EVALUATION DU

POINT DE VUE DES PATIENTS UTILISATEURS

THESE SOUTENUE PUBLIQUEMENT A LA FACULTE DE MEDECINE DE GRENOBLE*

Le 18 décembre 2013

POUR L'OBTENTION DU DOCTORAT EN MEDECINE

DIPLÔME D'ETAT

KNAPP Carole

Né(e) le 08/04/1983 à Nancy

DEVANT LE JURY COMPOSE DE

Président du jury : Monsieur le Professeur Pierre-Yves BENHAMOU

$\underline{\text { Directrice de thèse }}$ : Madame le Docteur Magalie BAUDRANT-BOGA

Membres du jury : Monsieur le Professeur Jean-Luc BOSSON

Monsieur le Professeur Philippe ZAOUI

*La Faculté de Médecine de Grenoble n'entend donner aucune approbation ni improbation aux opinions émises dans les thèses ; ces opinions sont considérées comme propres à leurs auteurs. 
Liste des Professeurs des Universités - Praticiens Hospitaliers 2012-2013

\begin{tabular}{|c|c|}
\hline Nom - Prénom & Discipline \\
\hline ALBALADEJO Pierre & Anesthésiologie réanimation \\
\hline ARVIEUX-BARTHELEMY Catherine & chirurgie générale \\
\hline BACONNIER Pierre & $\begin{array}{c}\text { Biostatiques, informatique médicale et } \\
\text { technologies de communication }\end{array}$ \\
\hline BAGUET Jean-Philippe & Cardiologie \\
\hline BALOSSO Jacques & Radiothérapie \\
\hline BARRET LUC & Médecine légale et droit de la santé \\
\hline BAUDAIN Philippe & Radiologie et imagerie médicale \\
\hline BEANI Jean-Claude & Dermato-vénéréologie \\
\hline BENHAMOU Pierre Yves & Endocrinologie, diabète et maladies métaboliques \\
\hline BERGER François & Biologie cellulaire \\
\hline BLIN Dominique & Chirurgie thoracique et cardio-vasculaire \\
\hline BONAZ Bruno & Gastro-entérologie, hépatologie, addictologie \\
\hline BOSSON Jean-Luc & $\begin{array}{c}\text { Biostatiques, informatique médicale et } \\
\text { technologies de communication }\end{array}$ \\
\hline BOUGEROI Thierry & Psychiatrie d'adultes \\
\hline BOUILLET Laurence & Médecine interne \\
\hline BRAMBILIA CHRISTIAN & Pneumologie \\
\hline BRAMBILLA Elisabeth & Anatomie et cytologie pathologiques \\
\hline BRICAULT Ivan & Radiologie et imagerie médicale \\
\hline
\end{tabular}




\begin{tabular}{|c|c|}
\hline BRICHON Pierre-Yves & Chirurgie thoracique et cardio-vasculaire \\
\hline CAHN Jean-Yves & Hématologie \\
\hline CARPENTIER Françoise & Thérapeutique, médecine d'urgence \\
\hline CARPENTIER Patrick & Chirurgie vasculaire, médecine vasculaire \\
\hline CESBRON Jean-Yves & Immunologie \\
\hline CHABARDES Stephan & Neurochirurgie \\
\hline CHABRE Olivier & Endocrinologie, diabète et maladies métaboliques \\
\hline CHAFFANJON Philippe & Anatomie \\
\hline CHAVANON Olivier & Chirurgie thoracique et cardio-vasculaire \\
\hline CHIQUET Christophe & Ophtalmologie \\
\hline CHIROSSEL Jean-Paul & Anatomie \\
\hline CINQUIN Philippe & $\begin{array}{c}\text { Biostatiques, informatique médicale et } \\
\text { technologies de communication }\end{array}$ \\
\hline COHEN Olivier & $\begin{array}{c}\text { Biostatiques, informatique médicale et } \\
\text { technologies de communication }\end{array}$ \\
\hline COUTURIER Pascal & Gériatrie et biologie du vieillissement \\
\hline CRACOWSKI Jean-Luc & $\begin{array}{c}\text { Pharmacologie fondamentale, pharmacologie } \\
\text { clinique }\end{array}$ \\
\hline DE GAUDEMARIS Régis & Médecine et santé au travail \\
\hline DEBILLON Thierry & Pédiatrie \\
\hline DEMATTEIS Maurice & Addictologie \\
\hline DEMONGEOT Jacques & $\begin{array}{c}\text { Biostatiques, informatique médicale et } \\
\text { technologies de communication }\end{array}$ \\
\hline DESCOTES Jean-Luc & Urologie \\
\hline ESTEVE François & Biophysique et médecine nucléaire \\
\hline FAGRET Daniel & Biophysique et médecine nucléaire \\
\hline FAUCHERON Jean-Luc & chirurgie générale \\
\hline
\end{tabular}




\begin{tabular}{|c|c|}
\hline FERRETTI Gilbert & Radiologie et imagerie médicale \\
\hline FEUERSTEIN Claude & Physiologie \\
\hline FONTAINE Eric & Nutrition \\
\hline FRANCOIS Patrice & $\begin{array}{c}\text { Epidémiologie, économie de la santé et } \\
\text { prévention }\end{array}$ \\
\hline GARBAN Frédéric & Hématologie, transfusion \\
\hline GAUDIN Philippe & Rhumatologie \\
\hline GAVAZZI Gaetan & Gériatrie et biologie du vieillissement \\
\hline GAY Emmanuel & Neurochirurgie \\
\hline GRIFFET Jacques & Chirurgie infantile \\
\hline HALIMI Serge & Nutrition \\
\hline HENNEBICQ Sylviane & Génétique et procréation \\
\hline HOFFMANN Pascale & Gynécologie obstétrique \\
\hline HOMMEL Marc & Neurologie \\
\hline JOUK Pierre-Simon & Génétique \\
\hline JUVIN Robert & Rhumatologie \\
\hline KAHANE Philippe & Physiologie \\
\hline KRACK Paul & Neurologie \\
\hline KRAINIK Alexandre & Radiologie et imagerie médicale \\
\hline LABARERE José & Département de veille sanitaire \\
\hline LANTUEJOUL Sylvie & Anatomie et cytologie pathologiques \\
\hline
\end{tabular}




\begin{tabular}{|c|c|}
\hline LE BAS Jean-François & Biophysique et médecine nucléaire \\
\hline LEBEAU Jacques & Chirurgie maxillo-faciale et stomatologie \\
\hline LECCIA Marie-Thérèse & Dermato-vénéréologie \\
\hline LEROUX Dominique & Génétique \\
\hline LEROY Vincent & Gastro-entérologie, hépatologie, addictologie \\
\hline LETOUBLON Christian & chirurgie générale \\
\hline LEVY Patrick & Physiologie \\
\hline LUNARDI Joël & Biochimie et biologie moléculaire \\
\hline MACHECOURT Jacques & Cardiologie \\
\hline MAGNE Jean-Luc & Chirurgie vasculaire \\
\hline MAITRE Anne & Médecine et santé au travail \\
\hline MAURIN Max & Bactériologie - virologie \\
\hline MERLOZ Philippe & Chirurgie orthopédique et traumatologie \\
\hline MORAND Patrice & Bactériologie - virologie \\
\hline MORO Elena & Neurologie \\
\hline MORO-SIBILOT Denis & Pneumologie \\
\hline MOUSSEAU Mireille & Cancérologie \\
\hline MOUTET François & $\begin{array}{c}\text { Chirurgie plastique, reconstructrice et } \\
\text { esthétique, brûlogie }\end{array}$ \\
\hline PALOMBI Olivier & Anatomie \\
\hline PASSAGIA Jean-Guy & Anatomie \\
\hline
\end{tabular}




\begin{tabular}{|c|c|}
\hline PAYEN DE LA GARANDERIE Jean-François & Anesthésiologie réanimation \\
\hline PELLOUX Hervé & Parasitologie et mycologie \\
\hline PEPIN Jean-Louis & Physiologie \\
\hline PERENNOU Dominique & Médecine physique et de réadaptation \\
\hline PERNOD Gilles & Médecine vasculaire \\
\hline PIOLAT Christian & Chirurgie infantile \\
\hline PISON Christophe & Pneumologie \\
\hline PLANTAZ Dominique & Pédiatrie \\
\hline POLACK Benoît & Hématologie \\
\hline PONS Jean-Claude & Gynécologie obstétrique \\
\hline RAMBEAUD Jacques & Urologie \\
\hline REYT Emile & Oto-rhino-laryngologie \\
\hline RIGHINI Christian & Oto-rhino-laryngologie \\
\hline ROMANET J. Paul & Ophtalmologie \\
\hline SARAGAGLIA Dominique & Chirurgie orthopédique et traumatologie \\
\hline SCHMERBER Sébastien & Oto-rhino-laryngologie \\
\hline SCHWEBEL Carole & Réanimation médicale \\
\hline SERGENT Fabrice & Gynécologie obstétrique \\
\hline SESSA Carmine & Chirurgie vasculaire \\
\hline STAHL Jean-Paul & Maladies infectieuses, maladies tropicales \\
\hline
\end{tabular}




\begin{tabular}{|c|c|}
\hline STANKE Françoise & Pharmacologie fondamentale \\
\hline TIMSIT Jean-François & Réanimation \\
\hline TONETTI Jérôme & Chirurgie orthopédique et traumatologie \\
\hline TOUSSAINT Bertrand & Biochimie et biologie moléculaire \\
\hline VANZETTO Gérald & Cardiologie \\
\hline VUILLEZ Jean-Philippe & Biophysique et médecine nucléaire \\
\hline WEIL Georges & $\begin{array}{c}\text { Epidémiologie, économie de la santé et } \\
\text { prévention }\end{array}$ \\
\hline ZAOUI Philippe & Néphrologie \\
\hline ZARSKI Jean-Pierre & Gastro-entérologie, hépatologie, addictologie \\
\hline
\end{tabular}


Liste des Maîtres de Conférences des Universités - Praticiens Hospitaliers 2012/2013

\begin{tabular}{|c|c|}
\hline Nom - Prénom & Discipline \\
\hline APTEL Florent & Ophtalmologie \\
\hline BOISSET Sandrine & Agents infectieux \\
\hline BONNETERRE Vincent & Médecine et santé au travail \\
\hline BOTTARI Serge & Biologie cellulaire \\
\hline BOUTONNAT Jean & Cytologie et histologie \\
\hline BOUZAT Pierre & Réanimation \\
\hline BRENIER-PINCHART M.Pierre & Parasitologie et mycologie \\
\hline BRIOT Raphaël & Thérapeutique, médecine d'urgence \\
\hline CALLANAN-WILSON Mary & Hématologie, transfusion \\
\hline DERANSART Colin & Physiologie \\
\hline DETANTE Olivier & Neurologie \\
\hline DIETERICH Klaus & Génétique et procréation \\
\hline DUMESTRE-PERARD Chantal & Immunologie \\
\hline EYSSERIC Hélène & Médecine légale et droit de la santé \\
\hline FAURE Julien & Biochimie et biologie moléculaire \\
\hline GILLOIS Pierre & $\begin{array}{c}\text { Biostatiques, informatique médicale et } \\
\text { technologies de communication }\end{array}$ \\
\hline GRAND Sylvie & Radiologie et imagerie médicale \\
\hline GUZUN Rita & $\begin{array}{c}\text { Endocrinologie, diabétologie, nutrition, } \\
\text { éducation thérapeutique }\end{array}$ \\
\hline LAPORTE François & Biochimie et biologie moléculaire \\
\hline LARDY Bernard & Biochimie et biologie moléculaire \\
\hline LARRAT Sylvie & Bactériologie, virologie \\
\hline LAUNOIS-ROLLINAT Sandrine & Physiologie \\
\hline MALLARET Marie-Reine & $\begin{array}{c}\text { Epidémiologie, économie de la santé et } \\
\text { prévention }\end{array}$ \\
\hline MAUBON Danièle & Parasitologie et mycologie \\
\hline
\end{tabular}




\begin{tabular}{|c|c|}
\hline MC LEER (FLORIN) Anne & Cytologie et histologie \\
\hline MOREAU-GAUDRY Alexandre & $\begin{array}{c}\text { Biostatiques, informatique médicale et } \\
\text { technologies de communication }\end{array}$ \\
\hline MOUCHET Patrick & Physiologie \\
\hline PACLET Marie-Hélène & Biochimie et biologie moléculaire \\
\hline PAYSANT François & Médecine légale et droit de la santé \\
\hline PELLETIER Laurent & Biologie cellulaire \\
\hline RAY Pierre & Génétique \\
\hline RIALLE Vincent & $\begin{array}{c}\text { Biostatiques, informatique médicale et } \\
\text { technologies de communication }\end{array}$ \\
\hline ROUX-BUISSON Nathalie & Biochimie, toxicologie et pharmacologie \\
\hline SATRE Véronique & Génétique \\
\hline STASIA Marie-Josée & Biochimie et biologie moléculaire \\
\hline TAMISIER Renaud & Physiologie \\
\hline
\end{tabular}




\section{REMERCIEMENTS}

Monsieur le Professeur Pierre-Yves BENHAMOU, vous me faites l'honneur de présider mon jury de thèse. Je vous remercie de m'avoir proposé ce travail, de l'attention que vous avez bien voulu y porter et de votre grande disponibilité.

Madame le Docteur Magalie BAUDRANT-BOGA, je vous remercie sincèrement d'avoir accepté de diriger ce travail. Merci pour vos relectures et vos conseils avisés, pour le temps que vous m'avez accordé et pour votre gentillesse.

Monsieur le Professeur Jean-Luc BOSSON, je vous remercie d'avoir accepté de juger ce travail.

Monsieur le Professeur Philippe ZAOUI, je vous remercie d'avoir accepté de juger ce travail.

Merci au Dr Isabelle DEBATY pour sa participation.

Merci à Muriel PIBOU pour son aide.

Je souhaite également remercier les patients qui m’ont accordé de leur temps.

Merci à ma famille, mon frère et ma belle-sœur.

Merci à Amaury pour ton aide précieuse, tes attentions et ta patience.

Merci à mes amis pour leur soutien et leurs conseils. Merci à Sophie pour m'avoir mise en relation avec mon Président de jury, pour ton amitié et ton écoute.

Merci aux médecins qui ont contribué à ma formation. 


\section{LISTE DES ABREVIATIONS}

ARS : Agence Régionale de Santé

CHU : Centre Hospitalier Universitaire

ENTRED : Échantillon National Témoin Représentatif des personnes Diabétiques

ETP : Education Thérapeutique du Patient

HAS : Haute Autorité de Santé

INDIGO : Intégrer les Notions essentielles sur le Diabète, l'Insuline et la Glycémie Online

INPES : Institut National de Prévention et d'Education pour la Santé

INSEE : Institut National de la Statistique et des Etudes Economiques

OMS : Organisation Mondiale de la Santé

UTEP : Unité Transversale d'Education du Patient 


\section{TABLE DES MATIERES}

I-Introduction

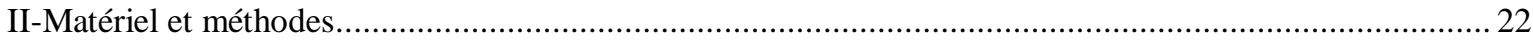

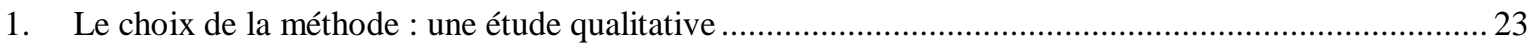

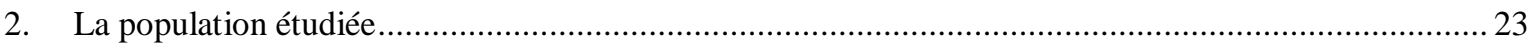

3. Conception du questionnaire de l'entretien dirigé .................................................................. 24

3.1. La grille d'analyse des outils d'intervention en éducation pour la santé de l'INPES....................24

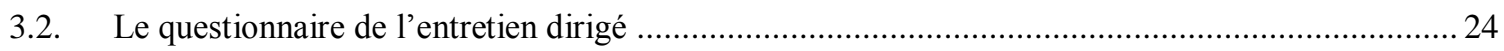

4. Conception du guide d'entretien (entretiens semi-dirigés) ...........................................................25

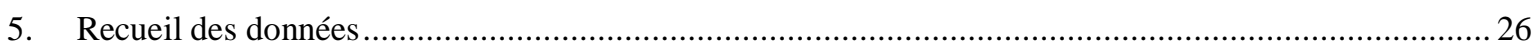

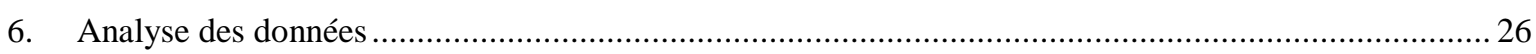

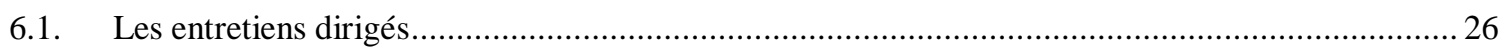

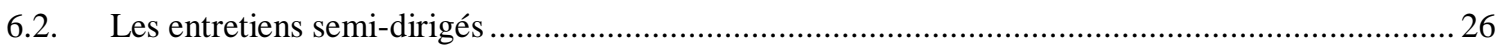

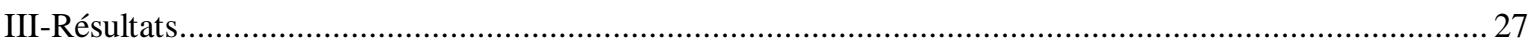

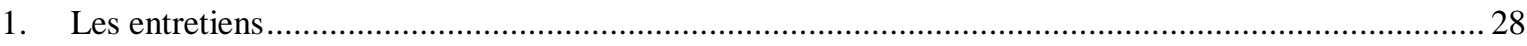

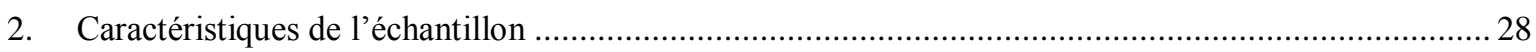

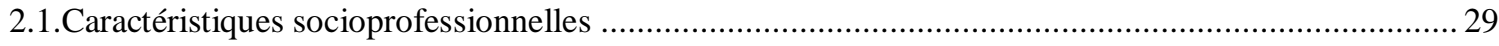

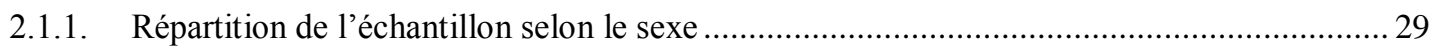

2.1.2. Répartition de l'échantillon selon l'âge ...................................................................29

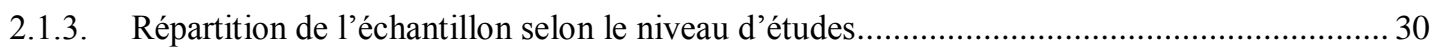

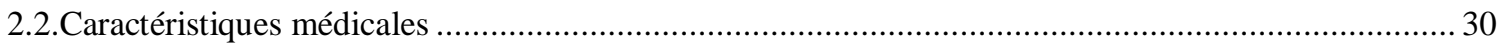

2.2.1. Répartition de l'échantillon selon l'ancienneté du diabète ................................................30

2.2.2. Répartition de l'échantillon selon le mode d'injection d'insuline .................................. 31

2.2.3. Répartition de l'échantillon selon le statut vis-à-vis de l'ETP........................................... 31

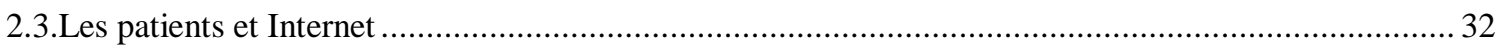

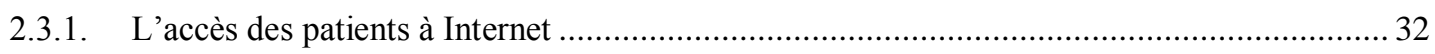

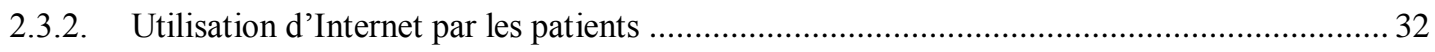

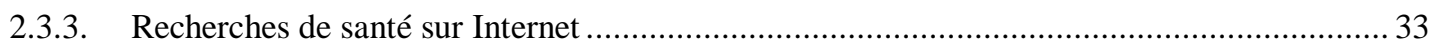

3. Analyse du questionnaire dirigé et des entretiens semi-dirigés ............................................... 34

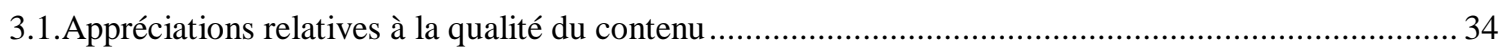

3.2.Appréciations relatives à la qualité pédagogique .............................................................. 40

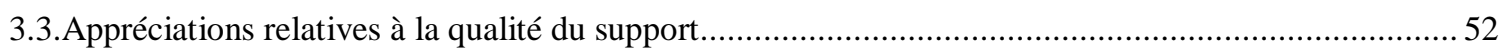

3.4.Appréciations relatives à la qualité de la conception..........................................................6. 61

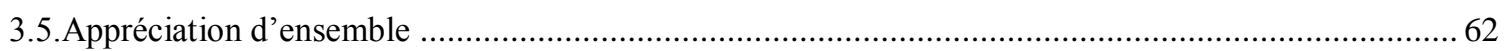

4. Analyse des appréciations chapitre par chapitre à partir des entretiens semi-dirigés .........................65

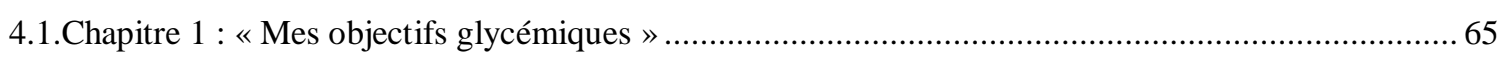

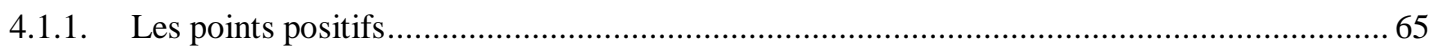

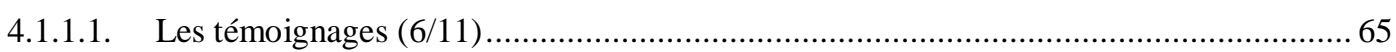

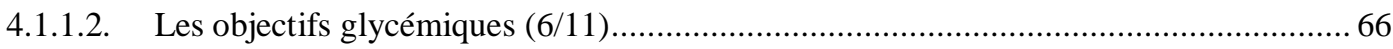

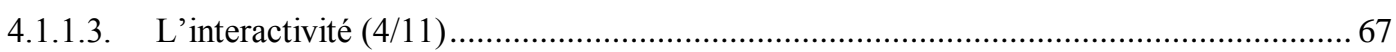


4.1.1.4. Autres réponses

4.1.2. Les points négatifs $(3 / 11)$

4.1.3. Les informations ont-elles répondu aux questions des patients interrogés concernant leurs objectifs glycémiques?

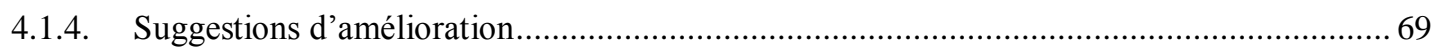

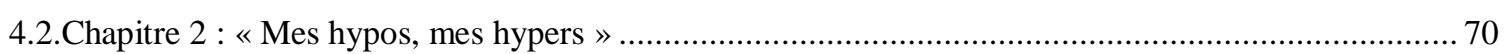

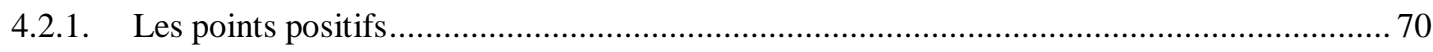

4.2.1.1. Chapitre 2.1 : «Comment savoir où j'en suis au niveau de ma glycémie ? » $(8 / 11) \ldots . . .70$

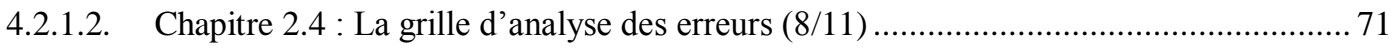

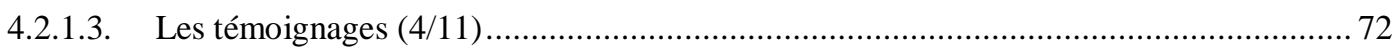

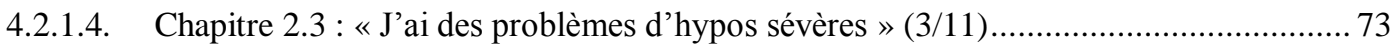

4.2.1.5. La notion de baisse du seuil de ressenti des hypoglycémies $(2 / 11)$......................... 73

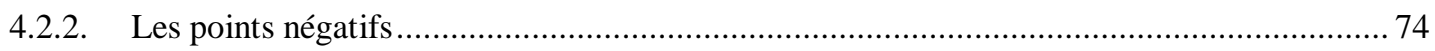

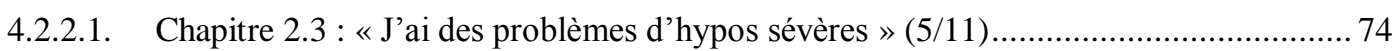

4.2.2.2. Chapitre 2.1 : «Comment savoir où j'en suis au niveau de ma glycémie ?» (2/11) ...... 74

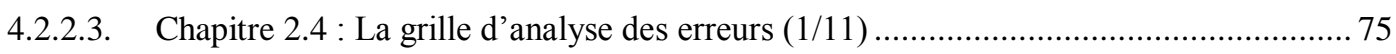

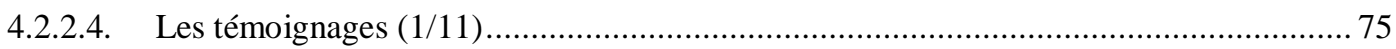

4.2.3. Les informations ont-elles répondu aux questions des patients interrogés concernant leurs

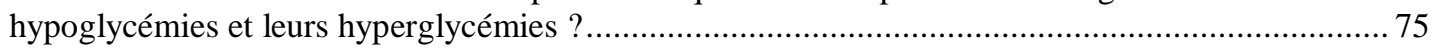

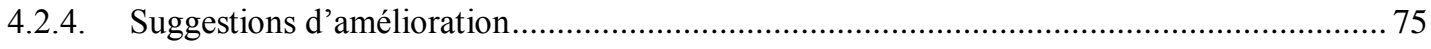

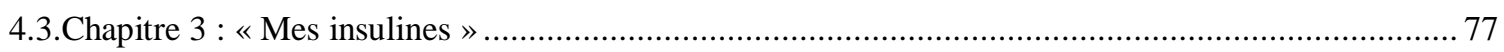

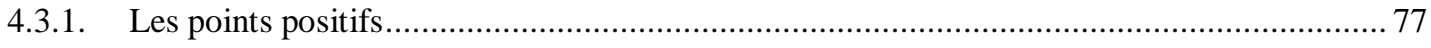

4.3.1.1. Chapitre 3.1 : « Comprendre mon traitement par insuline » (3/9) .............................. 77

4.3.1.2. Chapitre $3.3:$ « Comment définir mes doses d'insuline » (3/9) .............................. 77

4.3.1.3. Chapitre $3.4:$ «e manque d'insuline : hyperglycémie et acétone » $(3 / 9) \ldots \ldots \ldots \ldots \ldots . . . . .78$

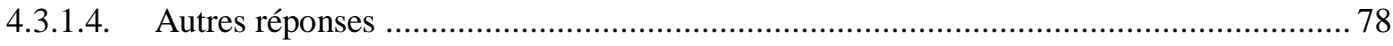

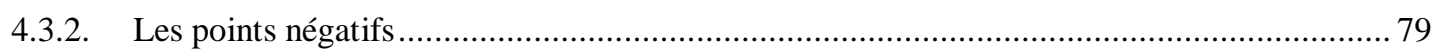

4.3.3. Les informations ont-elles répondu aux questions des patients interrogés concernant leurs

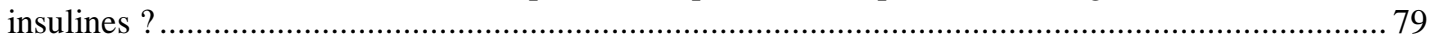

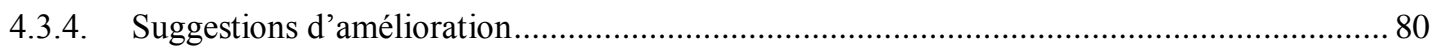

4.4.Quel est le chapitre ou le sous-chapitre qui a le moins intéressé les patients ?.................................81

4.5.Quel est le chapitre ou le sous-chapitre qui a le plus intéressé les patients ?....................................82

4.6.Impact de la participation à une action éducative antérieure et de l'ancienneté du diagnostic sur

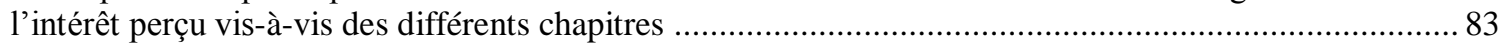

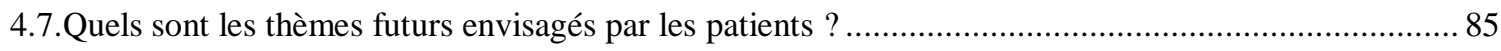

4.8.Quelle est la note de satisfaction globale attribuée par les patients ? ................................................86

5. Modalités d'utilisation du site INDIGO par les patients interrogés .............................................. 87

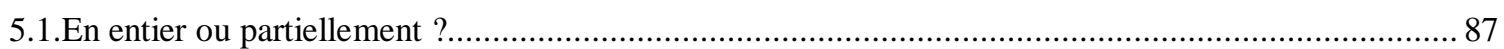

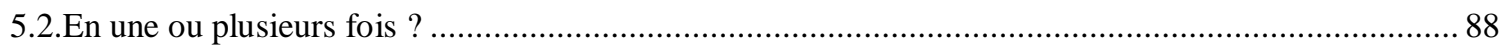

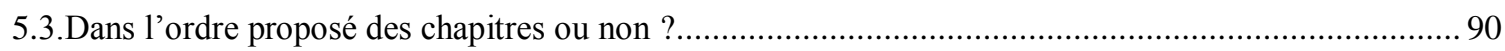

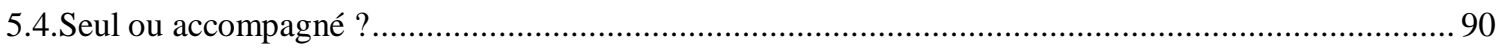

5.5.Les patients pensent-ils retourner sur le site INDIGO ? .......................................................... 91

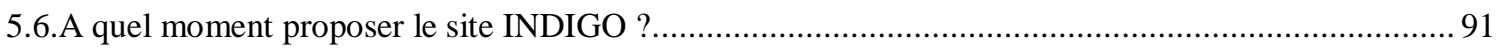


5.6.1. En fonction de l'ancienneté du diabète.................................................................... 91

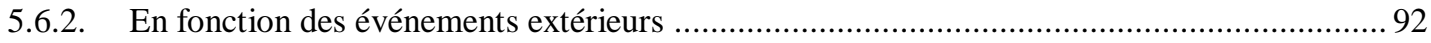

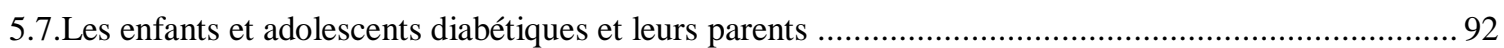

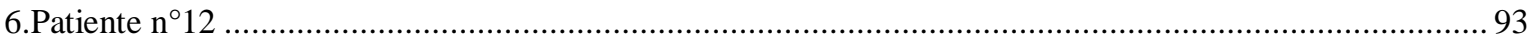

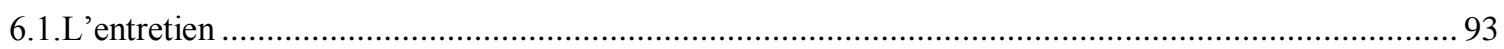

6.2.Caractéristiques socioprofessionnelles, médicales et utilisation d'Internet .................................93

6.3.Analyse du questionnaire dirigé et des entretiens semi-dirigés.....................................................93

6.3.1. Appréciations relatives à la qualité du contenu....................................................... 93

6.3.2. Appréciations relatives à la qualité pédagogique .....................................................93

6.3.3. Appréciations relatives à la qualité du support ......................................................... 94

6.3.4. Appréciations relatives à la qualité de la conception .................................................... 95

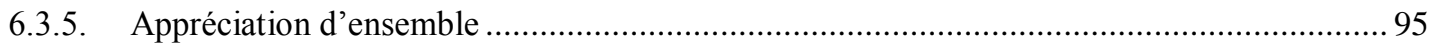

6.4.Analyse des appréciations chapitre par chapitre à partir des entretiens semi-dirigés.........................96

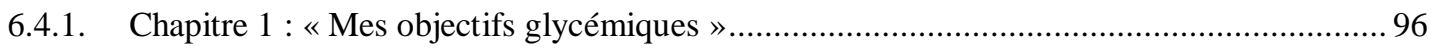

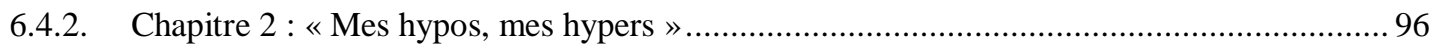

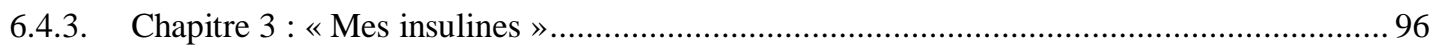

6.4.4. Quel est le chapitre ou le sous-chapitre qui a le moins intéressé la patiente $n^{\circ} 12$ ?............96

6.4.5. Quel est le chapitre ou le sous-chapitre qui a le plus intéressé la patiente $\mathrm{n}^{\circ} 12$ ? ...............97

6.4.6. Quels sont les thèmes futurs envisagés par la patiente $\mathrm{n}^{\circ} 12$ ?......................................... 97

6.4.7. Quelle est la note de satisfaction globale attribuée par la patiente $\mathrm{n}^{\circ} 12$ ?......................... 97

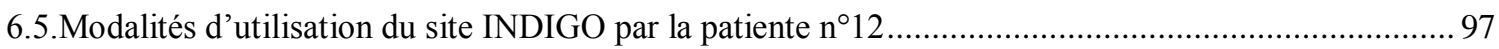

6.6.A quel moment proposer le site INDIGO d'après la patiente $\mathrm{n}^{\circ} 12$ ? ...........................................98

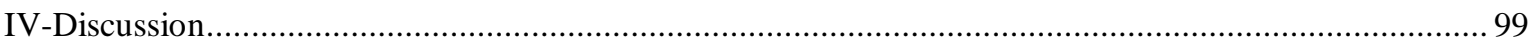

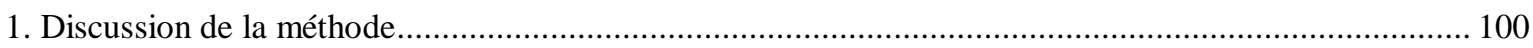

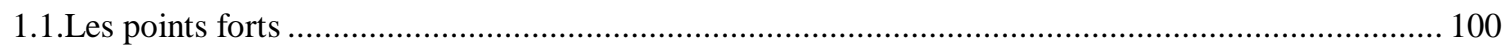

1.1.1. Les points forts de la méthode .......................................................................... 100

1.1.2. Les points forts de l'échantillon ............................................................................... 100

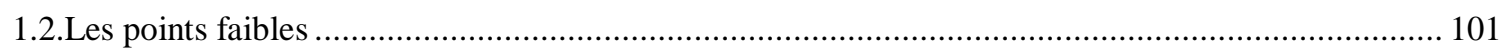

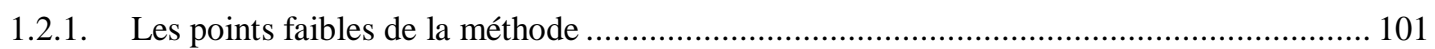

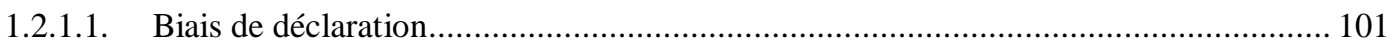

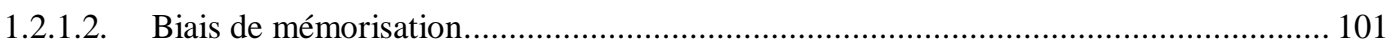

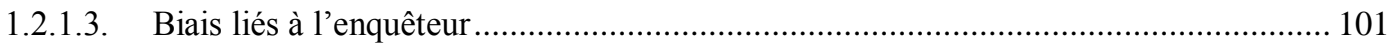

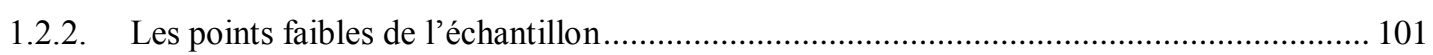

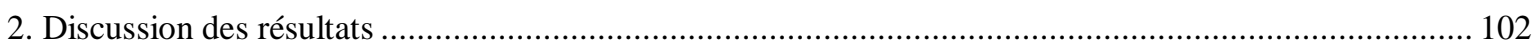

2.1.Qualités et freins perçus - Perspectives d'amélioration ........................................................... 102

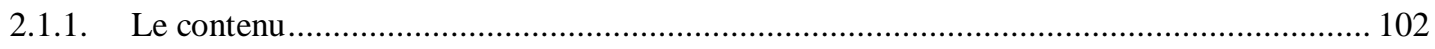

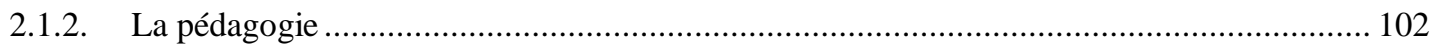

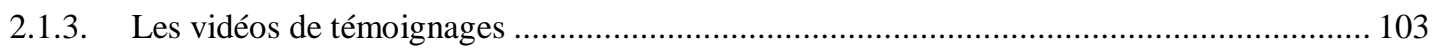

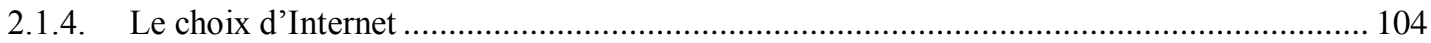

2.1.5. Le support technique ........................................................................................ 105

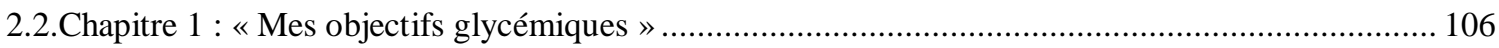

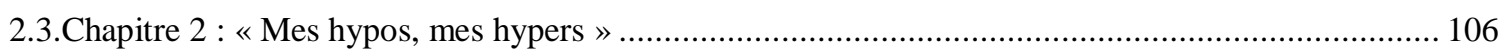


2.4.Chapitre $3:$ :Mes insulines »

2.5.Appréciation générale

2.6.Comment utiliser le site INDIGO ? 108

2.7.A quel moment utiliser le site INDIGO ? 109

2.8.Le site INDIGO et l'entourage des patients diabétiques de type 1 110

2.9.Au total 111

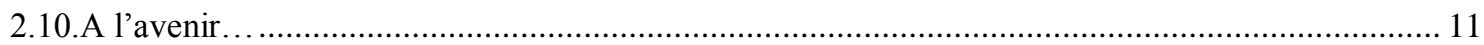

V-Conclusion 113

Bibliographie 117

Annexes 122

Retranscriptions 136 


\section{TABLE DES FIGURES}

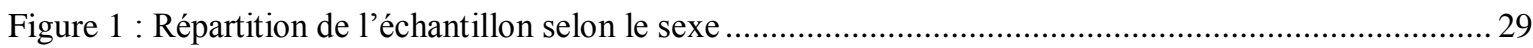

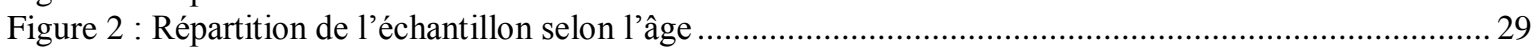

Figure 3 : Répartition de l'échantillon selon le niveau d'études......................................................... 30

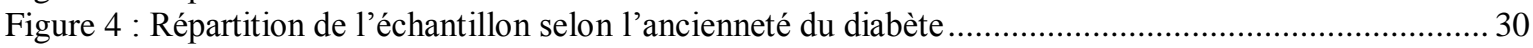

Figure 5 : Répartition de l'échantillon selon le mode d'injection d'insuline ........................................ 31

Figure 6 : Répartition de l'échantillon selon de statut vis-à-vis de l'ETP ............................................. 31

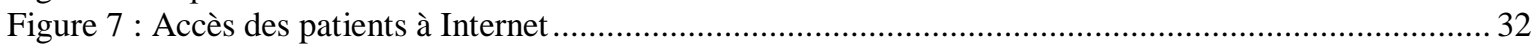

Figure 8 : Utilisation d'Internet et/ou de multimédia par les patients ................................................ 32

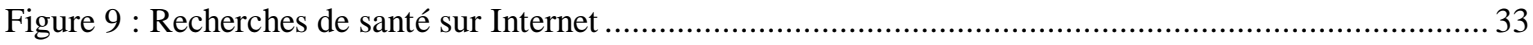

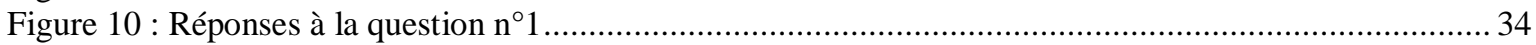

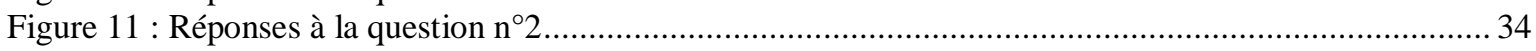

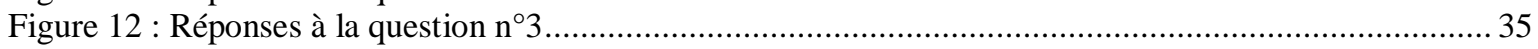

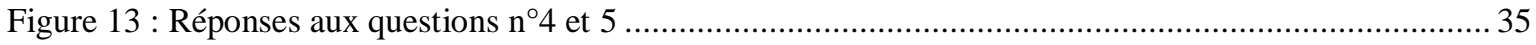

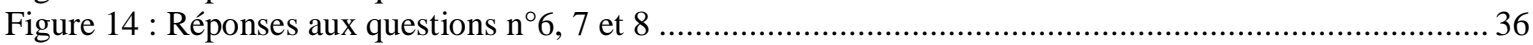

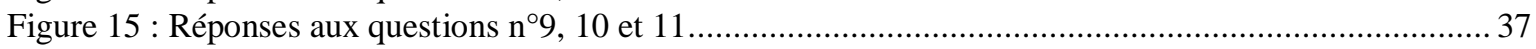

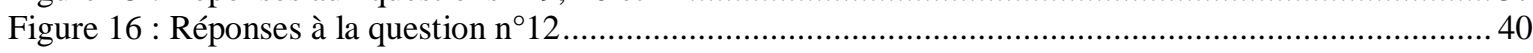

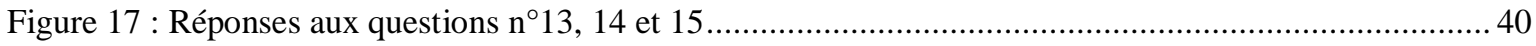

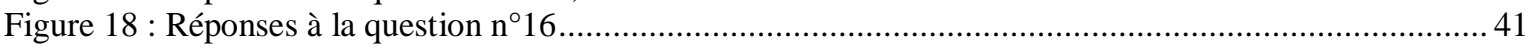

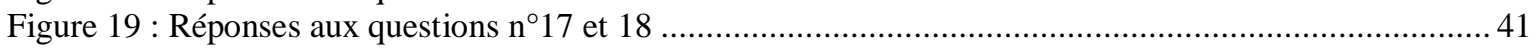

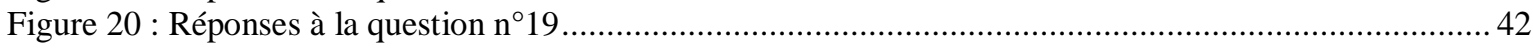

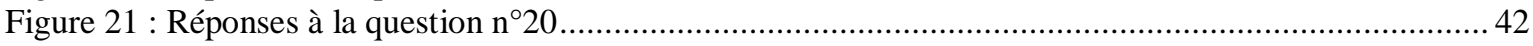

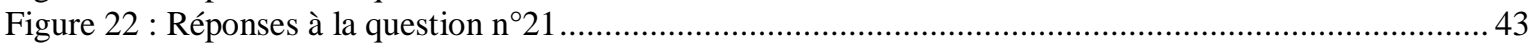

Figure 23 : A quels membres de leur entourage les patients ont-ils fait visionner ou conseilleraient-ils le site

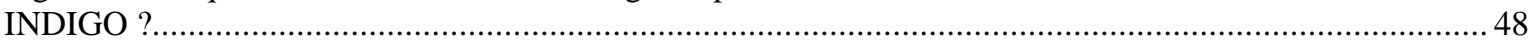

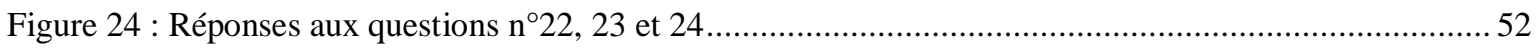

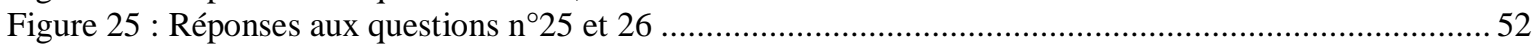

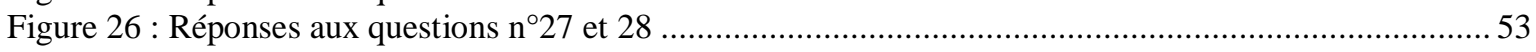

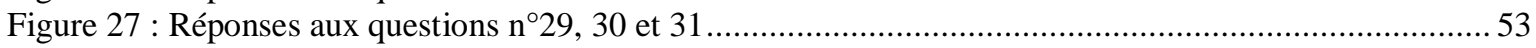

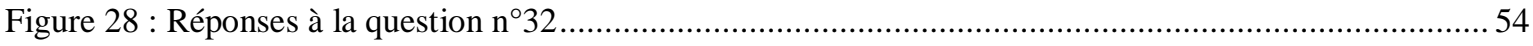

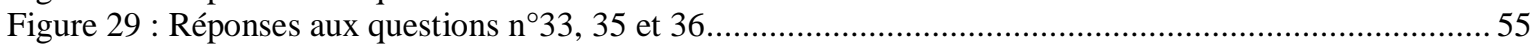

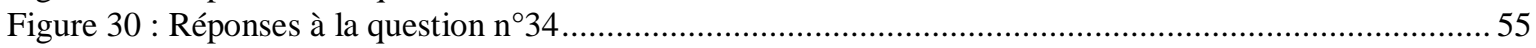

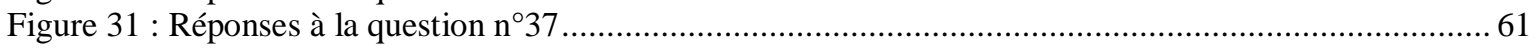

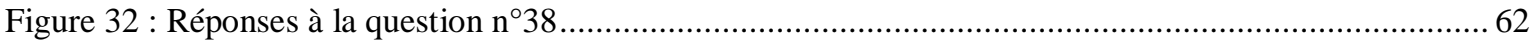

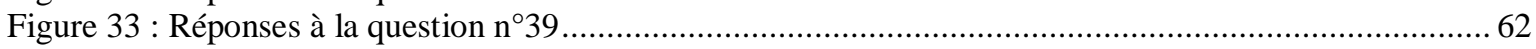

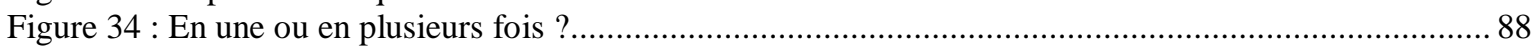

Figure 35 : Dans l'ordre proposé des chapitres ou non ? ................................................................90

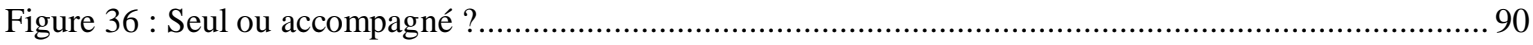

\section{TABLE DES TABLEAUX}

Tableau 1 : Outils utilisant Internet et les nouvelles technologies d'information et de communication destinés à des patients diabétiques de type 1 adultes disponibles en langue française en 2013 ...............................20

Tableau 2 : Caractéristiques socioprofessionnelles et médicales des patients interrogés ........................... 28

Tableau 3 : Impact de la participation à une action éducative antérieure sur l'intérêt perçu vis-à-vis des

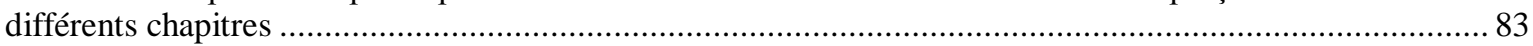

Tableau 4 : Notes de satisfaction globale attribuées par les patients de l'échantillon ...............................8 86

Tableau 5 : Caractéristiques socioprofessionnelles et médicales de la patiente $\mathrm{n}^{\circ} 12$................................93

Tableau 6 : Tableau récapitulatif des propositions faites par les patients (en bleu) et des perspectives envisagées par les soignants (en vert) 
I-Introduction 
En France, la prévalence du diabète traité pharmacologiquement en 2011 était estimée à 4,6\%, soit plus de 3 millions de personnes affectées. Elle est en constante augmentation (plus 5,4\% par an de 2000 à 2011). ${ }^{1}$ Selon l'étude ENTRED 2007-2010 ${ }^{2}$, le diabète de type 1 représentait un peu plus de 5,6\% des cas de diabète traité pharmacologiquement en métropole chez les plus de 18 ans en 2007. Plus de 180000 personnes sont concernées.

Le diabète est une maladie chronique qui altère la qualité et l'espérance de vie des personnes qui en sont atteintes. Elle nécessite une prise en charge adaptée visant à limiter ou éviter l'évolution vers les complications.

La chronicité de cette affection déplace le contrôle de la maladie du soignant vers le patient. L' " évolution favorable ou défavorable de la maladie chronique dépend donc de ce que le patient sait, fait et veut pour lui-même ». ${ }^{3}$

C'est pourquoi l'éducation thérapeutique du patient (ETP) est un élément indispensable dans la prise en charge des patients diabétiques.

Selon l'OMS : « [...] L'éducation thérapeutique du patient devrait permettre aux patients d'acquérir et de conserver les capacités et compétences qui les aident à vivre de manière optimale leur vie avec leur maladie. Il s'agit d'un processus permanent, intégré dans les soins et centré sur le patient. L'éducation implique des activités organisées de sensibilisation, d'information, d'apprentissage de l'autogestion et de soutien psychologique concernant la maladie, le traitement prescrit, les soins, le cadre hospitalier et de soins, les informations organisationnelles et les comportements de santé et de maladie. Elle vise à aider les patients et leur famille à comprendre la maladie et le traitement, coopérer avec les soignants, vivre plus sainement et maintenir ou améliorer leur qualité de vie $[. ..] \gg{ }^{4}$

La finalité de l'ETP est d'aider le patient à devenir «autonome », c'est-à-dire capable de prendre des décisions éclairées pour gérer au mieux sa maladie au quotidien et améliorer sa qualité de vie. ${ }^{5}$

L'ETP se doit d'être personnalisée, c'est-à-dire centrée sur le patient pour répondre avec pertinence et cohérence à ses besoins et demandes. ${ }^{6}$

La première expérience d'ETP a été menée en 1972 à Los Angeles par Leona Miller. Elle a formé 6000 malades diabétiques issus d'un milieu socioculturel défavorisé et a permis de réduire le nombre de journées d'hospitalisation de 5,4 jours par an et par 
patient à 1,7 jour. $^{7}$ Elle a ainsi démontré les effets positifs que peut avoir un programme éducatif sur la santé des patients diabétiques et sur le coût de leur prise en charge.

Depuis, de nombreuses études ont confirmé l'efficacité de l'ETP à différents niveaux : le contrôle glycémique, l'apparition de complications, la qualité de vie et le coût économique pour la société. $8,9,10$

En France, depuis quelques années, une succession de textes officiels marque la volonté politique de faire de l'ETP une priorité en matière de santé publique :

-En avril 2007, le Ministère de la Santé a publié le «Plan pour l'amélioration de la qualité de vie des personnes atteintes de maladies chroniques 2007-2011 ». ${ }^{11}$ Il comprend quinze mesures pour à répondre à quatre objectifs : aider chaque patient à mieux connaître sa maladie pour mieux la gérer, élargir la pratique médicale vers la prévention, faciliter la vie quotidienne des malades et mieux connaître les conséquences de la maladie sur la qualité de vie.

-En juin 2007, des recommandations professionnelles ont été publiées par la Haute Autorité de Santé (HAS). Il s'agit d'un guide méthodologique ${ }^{12}$ dont les objectifs sont de définir l'ETP, ses finalités, son organisation ; de décrire la démarche d'ETP, le contenu de ses étapes; de proposer une structuration d'un programme d'ETP; de proposer une évaluation du processus d'ETP et de proposer une réflexion pour évaluer l'efficacité de l'ETP.

-En 2008, le rapport de Christian Saout, Bernard Charbonnel et Dominique Bertrand «Pour une politique nationale d'éducation thérapeutique du patient ${ }^{13}$ a énoncé vingt quatre recommandations dans le but de développer l'ETP au niveau national.

-Depuis 2009, l'ETP est inscrite dans le Code de la Santé Publique comme un droit pour tout patient atteint d'une maladie chronique. Elle a fait l'objet de l'article 84 de la loi n²009-879 du 21 juillet 2009 portant réforme de l'hôpital et relative aux patients, à la santé et aux territoires (loi HPST) ${ }^{14}$ qui a pour objectif de définir le champ de l'ETP dans un souci de qualité et de proximité pour une meilleure prise en charge des personnes atteintes de maladies chroniques.

-En août 2010, deux décrets ${ }^{15,16}$ et deux arrêtés ${ }^{17,18}$ ont fixé les modalités d'autorisation des programmes d'ETP par les Agences Régionales de Santé (ARS) et les compétences requises pour les dispenser. En mai 2013, un décret ${ }^{19}$ et un arrêté ${ }^{20}$ ont procédé à une 
actualisation des compétences requises pour dispenser l'ETP. Ils définissent en outre les compétences requises pour la coordonner.

-En mars 2012, la HAS a proposé un guide méthodologique destiné à faciliter la réalisation de l'auto-évaluation annuelle d'un programme d'ETP. ${ }^{21}$ Il prépare ainsi à l'évaluation quadriennale de chaque programme d'ETP.

Cependant, malgré la diversité des offres éducatives proposées aux patients ; le plus souvent hospitalières mais aussi ambulatoires par l'intermédiaire des réseaux, maisons de santé, associations de patients etc. ; on constate encore qu'un grand nombre de patients n'a pas accès à un programme d'ETP. Diverses raisons peuvent être évoquées : listes d'attente, inégalités de répartition sur le territoire de l'offre d'ETP pouvant être à l'origine d'un éloignement géographique, contraintes familiales, contraintes professionnelles... ${ }^{22}$

Le développement des nouvelles technologies offre de nouvelles perspectives à l'ETP. $^{23,24}$

Selon l'enquête sur les technologies de l'information et de la communication datant du mois d'avril 2012, 78 \% des personnes âgées de 15 ans ou plus résidant en France métropolitaine déclarent avoir un accès à Internet à leur domicile (contre 54 \% en 2007 et $12 \%$ en 2000). Trois français sur quatre déclarent avoir utilisé Internet au cours des trois derniers mois (contre $56 \%$ en 2007) dont $79,7 \%$ tous les jours ou presque. Le développement accéléré de l'Internet mobile accompagne ces évolutions. En 2012, 40 \% des personnes résidant en France ont déjà surfé sur Internet en dehors de chez elles via un ordinateur portable, un téléphone portable ou un appareil de poche (contre $10 \%$ en 2007)..$^{25}$

Un accès à Internet développé, une couverture réseau étendue ainsi qu'une évolution des perceptions de la population face à ce nouveau média quel que soit l'âge de l'utilisateur ${ }^{25}$ permettent d'envisager l'utilisation de ces nouvelles technologies au sein d'une démarche éducative à destination des patients souffrants de maladie chronique et plus particulièrement des patients diabétiques de type 1 .

Le tableau 1 présente des exemples d'outils utilisant Internet et les nouvelles technologies d'information et de communication disponibles en langue française en 2013 
qui pourraient être utilisés comme support au sein de programmes éducatifs pour les patients diabétiques de type 1 adultes,. (Tableau non exhaustif)

\begin{tabular}{|c|c|c|}
\hline \multirow{6}{*}{ Sites Internet } & DiabSurf & $\begin{array}{l}\text { Site crée par un diabétologue, informations écrites, quiz, } \\
\text { forum : www.diabsurf.com }\end{array}$ \\
\hline & $\begin{array}{l}\text { Site de l'association } \\
\text { française des } \\
\text { diabétiques }\end{array}$ & $\begin{array}{l}\text { Informations écrites, témoignages par écrit, recettes, } \\
\text { quiz... : www.afd.asso.fr }\end{array}$ \\
\hline & Diabète Québec & $\begin{array}{l}\text { Informations écrites, liens vers des vidéos de } \\
\text { professionnels et de patients, témoignages par écrit, } \\
\text { recettes... : www.diabete.qc.ca }\end{array}$ \\
\hline & $\begin{array}{l}\text { Association } \\
\text { Française des } \\
\text { femmes diabétiques }\end{array}$ & $\begin{array}{l}\text { Site consacré à la grossesse chez les femmes diabétiques : } \\
\text { informations, témoignages par écrit, forum... : } \\
\text { http://femmesdiabetiques.com }\end{array}$ \\
\hline & $\begin{array}{l}\text { La pompe à } \\
\text { insuline, parlons } \\
\text { en! }\end{array}$ & $\begin{array}{l}\text { Site de l'Association Française des Diabétiques consacré à } \\
\text { la pompe à insuline : informations écrites, témoignages par } \\
\text { écrit, quiz... : http://pompeainsuline.afd.asso.fr }\end{array}$ \\
\hline & Le pied diabétique & $\begin{array}{l}\text { Site de l'Association Belge du Diabète consacré au pied } \\
\text { diabétique : www.piediab.org }\end{array}$ \\
\hline \multirow{3}{*}{$\begin{array}{c}\text { Jeux sérieux ou } \\
\text { « serious games » } \\
\text { (basés sur la } \\
\text { technologie des jeux } \\
\text { vidéo) }\end{array}$} & $\begin{array}{l}\text { Méli-mélo } \\
\text { glucidique }\end{array}$ & $\begin{array}{l}\text { Jeux consacrés aux connaissances diététiques : } \\
\text { www.glucifer.net }\end{array}$ \\
\hline & L'affaire Birman & $\begin{array}{l}\text { Jeu d'aventure intégrant les principes de l'insulinothérapie } \\
\text { fonctionnelle. L'étude LUDIDIAB }{ }^{26} \text { a montré une } \\
\text { amélioration des connaissances à } 0 \text { et } 6 \text { mois : } \\
\text { www.glucifer.net }\end{array}$ \\
\hline & Time Out & $\begin{array}{l}\text { Jeu d'aventure dont le héros est traité par pompe à insuline } \\
\text { et adapte ses doses selon la technique de l'insulinothérapie } \\
\text { fonctionnelle : } \underline{w w} \text {.glucifer.net }\end{array}$ \\
\hline \multirow[t]{2}{*}{$\begin{array}{l}\text { Applications pour } \\
\text { smartphone }\end{array}$} & $\begin{array}{l}\text { Guide voyage pour } \\
\text { la personne } \\
\text { diabétique }\end{array}$ & $\begin{array}{l}\text { Application d'aide aux préparatifs de voyage apportant de } \\
\text { plus des outils pratiques une fois à destination : liste des } \\
\text { documents nécessaires pour les contrôles de sécurité, noms } \\
\text { des différentes insulines disponibles dans le pays de } \\
\text { destination, stratégies d'actions face à différentes situations } \\
\text { comme lors d'un décalage horaire, d'une prise de repas } \\
\text { retardée... }\end{array}$ \\
\hline & Diabé'TEAM & $\begin{array}{l}\text { Application permettant le calcul du nombre de glucides } \\
\text { contenus dans le repas et de la dose d'insuline à injecter qui } \\
\text { se veut davantage être une aide pratique au quotidien qu'un } \\
\text { outil de suivi thérapeutique. Egalement disponible sur } \\
\text { Internet : www.diabeteam.org }\end{array}$ \\
\hline
\end{tabular}

Tableau 1 : Outils utilisant Internet et les nouvelles technologies d'information et de communication destinés à des patients diabétiques de type 1 adultes disponibles en langue française en 2013

Dans ce contexte, le service de diabétologie du Centre Hospitalier Universitaire de Grenoble a créé un outil d'ETP multimédia d'aide à la prise en charge du diabète à destination des patients diabétiques de type 1, accessible par Internet et sur CD-Rom : l'outil INDIGO (pour Intégrer les Notions essentielles sur le Diabète, l'Insuline et la Glycémie Online). Le site Internet est consultable à l'adresse : http://www.indigodiabete.fr en utilisant les navigateurs Safari ou Chrome.

L'outil INDIGO se distingue des outils présentés dans le tableau 1 par sa forme et par ses objectifs qui sont de permettre au patient d'identifier par lui-même ses réels besoins 
de soins tout en lui apportant des connaissances théoriques, des points de repère et des outils qui lui sont nécessaires au quotidien et en lui proposant une utilisation pratique immédiate des ressources qui lui sont proposées. ${ }^{27}$

Les données médicales qui y sont délivrées ont été validées par un comité scientifique pluridisciplinaire comportant une diététicienne et un médecin formés à l'ETP, un médecin expert en diabète de type 1 et une interne en diabétologie. Deux patients diabétiques de type 1 ont été intégrés à cette équipe dans le souci d'adapter l'outil aux demandes réelles des patients. ${ }^{27}$

Au moment où cette étude a été menée, il était composé de trois chapitres : (cf. Sommaire du site en Annexe 1)

- «Mes objectifs glycémiques »

- «Mes hypos, mes hypers »

- «Mes insulines »

Deux chapitres étaient en cours d'élaboration :

- «Mon alimentation »

- «Mes activités physiques »

La totalité du site se parcourt en 3 heures $1 / 2$ environ mais les différents chapitres et sous-chapitres peuvent être consultés à la carte et en plusieurs fois. Les informations y sont délivrées par une voix off. Elles sont parfois lisibles à l'écran simultanément ou bien illustrées par des schémas, des graphiques, des photographies ou des séquences vidéo. On y trouve des interviews de patients filmés dans leur quotidien qui livrent leurs points de vue et leurs expériences de la maladie. Des animations nécessitant la participation du patient (cases à cocher, quizz etc.) sont également proposées. (cf. Annexe 2)

La démarche d'ETP étant par définition basée sur les besoins et attentes des patients, il semble nécessaire d'étudier ce qu'ils pensent de l'outil INDIGO.

L'objectif principal de cette étude est d'évaluer si, du point de vue des patients utilisateurs, l'outil correspond aux critères de qualité d'un outil d'éducation pour la santé de l'Institut National de Prévention et d'Education pour la Santé (INPES). ${ }^{28}$

Les objectifs secondaires sont d'évaluer les modalités d'utilisation de l'outil INDIGO par les patients, de recueillir leurs appréciations et de préciser les perspectives d'amélioration de l'outil. 
II-Matériel et méthodes 


\section{Le choix de la méthode : une étude qualitative}

Pour répondre à la question «L'outil INDIGO satisfait-il aux critères de qualité d'un outil d'éducation pour la santé édictés par $1^{1} \operatorname{INPES}^{28}$ ? », les données ont été collectées à partir d'entretiens dirigés. L'entretien dirigé consiste à interroger le patient à l'oral au moyen d'un questionnaire structuré. Les questions sont de type dichotomique (oui/non) ou sous forme d'échelles de Likert (échelle mesurant le degré d'accord ou de désaccord vis-à-vis d'une affirmation, par exemple : tout à fait d'accord, plutôt d'accord, plutôt pas d'accord, pas du tout d'accord) ou d'échelles numériques (note entre 1 et 10). Leur ordre est défini à l'avance. Ce type d'entretien permet au patient interrogé de se positionner quant aux critères de qualité proposés mais lui laisse peu de liberté d'expression. ${ }^{29}$

Ensuite, des entretiens semi-dirigés individuels ont été menés afin de compléter les données par une exploration approfondie du point de vue des patients et de leur expérience avec l'outil. Alors que le questionnaire provoque une réponse, l'entretien semi-dirigé permet la construction d'un discours. Cette méthode est pertinente lorsque l'on souhaite collecter des propos exprimant des opinions. ${ }^{30}$

\section{La population étudiée}

Les critères d'inclusion des patients étaient les suivants : être diabétique de type 1 diagnostiqué depuis moins de trois ans, être suivi au CHU de Grenoble, être âgé de plus de 18 ans et avoir été informé de l'existence de l'outil INDIGO" en consultation. Le fait d'avoir reçu une éducation thérapeutique «conventionnelle» n'était pas un critère

d'exclusion. (" seule la version «site Internet» de l'outil INDIGO a été présentée aux patients et utilisée par eux pour cette étude.)

Dans un travail exploratoire comme celui-ci, sans hypothèse à priori, c'est la diversité maximale des profils des patients interrogés à l'intérieur de la population cible qui est recherchée. ${ }^{31}$

Une étude qualitative ne cherche pas à extrapoler les résultats obtenus à l'ensemble de la population, il n'y a donc pas de notion de représentativité. ${ }^{32}$

Le nombre d'entretiens à effectuer est déterminé par l'apparition de redondances dans les propos des personnes interrogées. Ceci correspond au critère de la saturation théorique. ${ }^{33}$ 
Un courrier présentant l'étude a été adressé aux patients inclus par voie postale, ainsi que par courrier électronique pour les patients dont l'adresse électronique était connue (cf. Annexe 3). Ensuite, ils ont été contactés par téléphone pour convenir d'un rendez-vous qui pouvait se dérouler à leur convenance à leur domicile, au CHU, par téléphone ou par Skype.

\section{Conception du questionnaire de l'entretien dirigé}

Le questionnaire a été conçu en se basant sur la grille d'analyse des outils d'intervention en éducation pour la santé proposée par l'INPES. ${ }^{28}$

\subsection{La grille d'analyse des outils d'intervention en éducation pour la santé de l'INPES (cf. Annexe 4)}

Un référentiel de critères de qualité a été élaboré à partir d'une analyse de la littérature par un groupe d'experts en évaluation et en éducation pour la santé. Il définit les 31 critères essentiels de qualité des outils d'intervention en éducation pour la santé, auxquels s'ajoutent 30 critères importants et 4 critères mineurs. Ces 65 critères ont été répartis en cinq catégories : qualité du contenu, qualité pédagogique, qualité du support, qualité de la conception et appréciation d'ensemble. Ils ont ensuite été transformés en questions qui permettent de vérifier l'adéquation de l'outil examiné avec chacun des critères de qualité. C'est ainsi qu'a été conçue par l'INPES la grille d'analyse des outils d'intervention en éducation pour la santé.

\subsection{Le questionnaire de l'entretien dirigé}

Le questionnaire de l'entretien dirigé (cf. Annexe 5) a été élaboré et validé par un groupe d'experts multi-professionnel et pluridisciplinaire constitué d'un diabétologue, d'un médecin appartenant à l'Unité Transversale d'Education du Patient (UTEP), d'un pharmacien hospitalier membre de l'UTEP et d'un médecin généraliste.

Les 65 critères de qualité des outils d'intervention en éducation pour la santé ont été revus par chaque membre du groupe d'experts indépendamment. Leur objectif était de se positionner quant à la pertinence de chacun des critères par rapport à l'évaluation d'un outil d'ETP.

Ensuite une séance de «brainstorming » a été réalisée afin de prendre une décision négociée concernant les critères pour lesquelles une discordance existait entre les experts. 
Les critères considérés comme non applicables au site INDIGO (par exemple «Qualité des matériaux : solidité et robustesse »), qui n'étaient pas évaluables par le patient lui-même (par exemple «Les informations présentes dans cet outil sont-elles d'actualité ? ») ou qui présentaient une redondance par rapport à un autre critère (par exemple «Pertinence du choix du support au regard du sujet traité ? » et «Pertinence du choix du support au regard des destinataires ? ») n'ont pas été retenus.

Certains des critères retenus ont été reformulés pour être adaptés à l'étude en cours (par exemple la question «Quelle est, selon vous, la pertinence du contenu par rapport au thème principal de l'outil ? » est devenue «Quelle est, selon vous, la pertinence de la présence du chapitre Mes objectifs glycémiques ?, du chapitre Mes hypos, mes hypers ? et du chapitre Mes insulines? ») ou pour faciliter la compréhension par les patients (par exemple la question «Avez-vous eu le sentiment que l'outil comportait un contenu inacceptable au regard de l'éthique ? » est devenue «Avez-vous eu le sentiment que le site INDIGO comportait un contenu inacceptable au regard de vos valeurs ? »). Au total, le questionnaire ainsi élaboré comptait 39 questions.

Il a ensuite été testé auprès de sept personnes de profils différents, afin d'évaluer la clarté et l'intelligibilité des questions posées. Il s'agissait de quatre professionnels de santé et de trois personnes n'appartenant pas au milieu médical. Aucun ne faisait partie du projet en cours. Ce pré-test a permis d'apporter quelques modifications et d'évaluer la faisabilité de la passation du questionnaire, notamment le temps nécessaire pour être complété.

\section{Conception du guide d'entretien (entretiens semi-dirigés)}

Le guide d'entretien (cf. Annexe 6) précise les thèmes à aborder au cours de l'entretien semi-dirigé.

L'entretien semi-dirigé débute par une question ouverte. Ensuite les questions correspondant aux différentes thématiques prédéfinies ne sont pas posées de manière systématique mais abordées à des moments opportuns de l'entretien qui peuvent être amorcés par le patient lui-même ou suite à une reformulation de l'enquêtrice selon la technique de l'écoute active ou écoute bienveillante. ${ }^{34}$

Il a été élaboré et validé par le même groupe d'experts et selon la même méthodologie que pour le questionnaire dirigé : une phase indépendante puis une séance de «brainstorming ». 


\section{Recueil des données}

L'ensemble des entretiens a été enregistré à l'aide d'un dictaphone numérique après avoir obtenu l'accord oral des patients interrogés. Chaque entretien semi-dirigé a été retranscrit intégralement. Le matériel non verbal (les hésitations, les rires, les pauses) a été noté également.

\section{Analyse des données}

\subsection{Les entretiens dirigés}

Les données issues de l'entretien dirigé ont été collectées au sein d'un tableau Excel de manière anonyme. Une analyse descriptive a été réalisée.

\subsection{Les entretiens semi-dirigés}

Une analyse thématique a été effectuée. Les différentes étapes de l'analyse thématique sont la retranscription intégrale des entretiens ; l'étude des retranscriptions individuellement afin de faire émerger les unités de sens ; la confrontation des unités de sens permettant de distinguer les thèmes généraux et enfin la classification de ces thèmes en thèmes principaux et thèmes secondaires. ${ }^{35}$ D'après Blanchet et Gotman, ce genre d'analyse « défait la singularité du discours et découpe transversalement, ce qui, d'un entretien à l'autre, se réfère au même thème. Elle ignore ainsi la cohérence singulière de l'entretien, et cherche une cohérence thématique inter-entretiens. La manipulation thématique consiste ainsi à jeter l'ensemble des éléments signifiants dans une sorte de sac à thèmes qui détruit définitivement l'architecture cognitive et affective des personnes singulières $\gg .{ }^{30}$

Pour augmenter la robustesse de l'analyse, les entretiens ont été relus et analysés indépendamment par une autre personne appartenant au projet, formée à l'analyse thématique. Ensuite, l'ensemble des verbatims dont le classement était discordant a fait l'objet d'une négociation et d'un choix collégial lors d'une réunion commune. 


\section{III-Résultats}




\section{Les entretiens}

Treize patients correspondaient aux critères d'inclusion et ont été contactés. Deux patients ont refusé de participer à l'étude.

A la convenance des patients, sept entretiens se sont déroulés dans le service de diabétologie du CHU de Grenoble, aucun au domicile des patients et quatre ont été menés par téléphone.

La durée des entretiens variait de 27 minutes à 47 minutes avec une moyenne de 38 minutes par entretien.

\section{Caractéristiques de l'échantillon}

Les caractéristiques socioprofessionnelles et médicales des patients ayant participé à l'étude sont résumées dans le tableau 2.

\begin{tabular}{|c|c|c|c|c|c|c|c|}
\hline & Sexe & Age & $\begin{array}{l}\text { Niveau } \\
\text { d'études }\end{array}$ & Profession & $\begin{array}{l}\text { Ancienneté } \\
\text { du diabète }\end{array}$ & Stylo/Pompe & $\begin{array}{c}\text { Participation } \\
\text { ETP } \\
\text { antérieure }\end{array}$ \\
\hline $\begin{array}{c}\text { Patient } \\
n^{\circ} 1 \\
\end{array}$ & Femme & $\begin{array}{c}21 \\
\text { ans }\end{array}$ & $\mathrm{Bac}$ & Etudiante & 8 mois & Stylo & Non \\
\hline $\begin{array}{c}\text { Patient } \\
n^{\circ} 2\end{array}$ & Homme & $\begin{array}{c}22 \\
\text { ans }\end{array}$ & $\begin{array}{c}\text { Bac }+3 \text { et } \\
\text { plus }\end{array}$ & $\begin{array}{l}\text { Etudiant } \\
\text { ingénieur }\end{array}$ & 9 mois & Stylo & $\begin{array}{c}3 \text { jours en } \\
\text { groupe en } \\
2013\end{array}$ \\
\hline $\begin{array}{c}\text { Patient } \\
n^{\circ} 3 \\
\end{array}$ & Homme & $\begin{array}{c}33 \\
\text { ans }\end{array}$ & $\begin{array}{c}\text { Bac }+3 \text { et } \\
\text { plus }\end{array}$ & $\begin{array}{l}\text { Ingénieur - } \\
\text { Chercheur }\end{array}$ & 3 mois & Stylo & Non \\
\hline $\begin{array}{c}\text { Patient } \\
n^{\circ} 4\end{array}$ & Homme & $\begin{array}{l}40 \\
\text { ans }\end{array}$ & $\begin{array}{c}\text { Bac }+3 \text { et } \\
\text { plus }\end{array}$ & $\begin{array}{l}\text { Ingénieur en } \\
\text { électronique }\end{array}$ & 3 mois & Stylo & Non \\
\hline $\begin{array}{l}\text { Patient } \\
n^{\circ} 5\end{array}$ & Femme & $\begin{array}{c}19 \\
\text { ans }\end{array}$ & $\mathrm{Bac}+2$ & $\begin{array}{c}\text { Etudiante en } \\
\text { espagnol }\end{array}$ & 21 mois & Stylo & $\begin{array}{c}3 \text { jours en } \\
\text { groupe en } \\
11 / 2012\end{array}$ \\
\hline $\begin{array}{c}\text { Patient } \\
n^{\circ} 6 \\
\end{array}$ & Femme & $\begin{array}{l}44 \\
\text { ans }\end{array}$ & $\mathrm{Bac}$ & $\begin{array}{l}\text { Aide médico- } \\
\text { psychologique }\end{array}$ & 2 mois & Stylo & Non \\
\hline $\begin{array}{c}\text { Patient } \\
n^{\circ} 7\end{array}$ & Femme & $\begin{array}{c}35 \\
\text { ans }\end{array}$ & $\begin{array}{c}\text { Bac }+3 \text { et } \\
\text { plus }\end{array}$ & $\begin{array}{c}\text { Chargée de } \\
\text { communication }\end{array}$ & 11 mois & Stylo & $\begin{array}{c}3 \text { jours en } \\
\text { groupe en } \\
06 / 2013\end{array}$ \\
\hline $\begin{array}{c}\text { Patient } \\
\mathbf{n}^{\circ} 8 \\
\end{array}$ & Homme & $\begin{array}{l}27 \\
\text { ans }\end{array}$ & $\begin{array}{c}\text { Bac }+3 \text { et } \\
\text { plus }\end{array}$ & $\begin{array}{l}\text { Etudiant en } \\
\text { économie }\end{array}$ & 5 mois & Stylo & Non \\
\hline $\begin{array}{c}\text { Patient } \\
n^{\circ} 9 \\
\end{array}$ & Femme & $\begin{array}{l}23 \\
\text { ans }\end{array}$ & $\mathrm{Bac}$ & Intérimaire & 7 mois & Pompe & Non \\
\hline $\begin{array}{l}\text { Patient } \\
n^{\circ} 10\end{array}$ & Femme & $\begin{array}{l}40 \\
\text { ans }\end{array}$ & $\mathrm{Bac}$ & $\begin{array}{l}\text { Clerc aux } \\
\text { formalités }\end{array}$ & 9 mois & Stylo & $\begin{array}{c}3 \text { jours en } \\
\text { groupe en } 2013\end{array}$ \\
\hline $\begin{array}{l}\text { Patient } \\
n^{\circ} 11\end{array}$ & Femme & $\begin{array}{c}21 \\
\text { ans }\end{array}$ & $\begin{array}{c}\text { Bac }+3 \text { et } \\
\text { plus }\end{array}$ & Etudiante & 35 mois & Stylo & $\begin{array}{l}1 \text { jour en } \\
\text { groupe en } 2011\end{array}$ \\
\hline
\end{tabular}

Tableau 2 : Caractéristiques socioprofessionnelles et médicales des patients interrogés 


\subsection{Caractéristiques socioprofessionnelles}

\subsubsection{Répartition de l'échantillon selon le sexe}

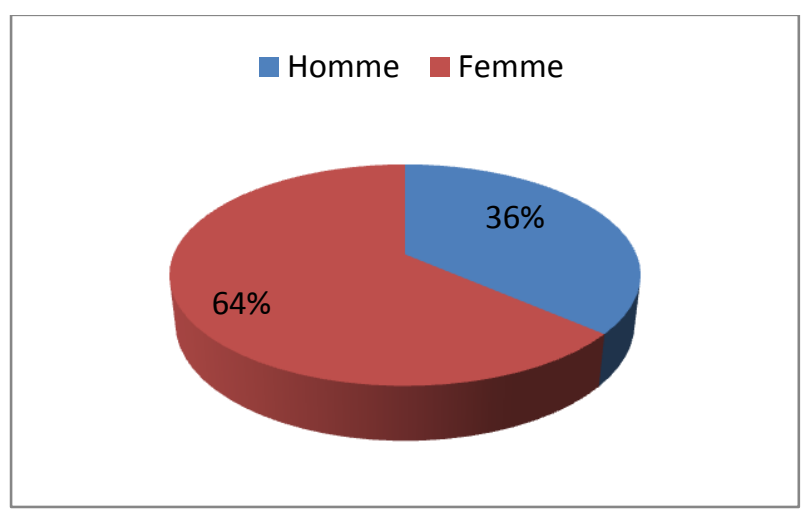

Figure 1 : Répartition de l'échantillon selon le sexe

L'échantillon était constitué de 7 femmes et de 4 hommes.

\subsubsection{Répartition de l'échantillon selon l'âge}

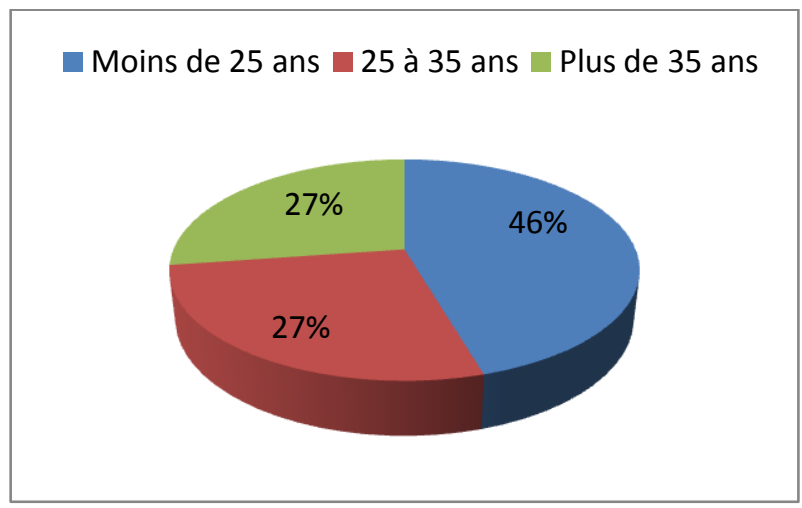

Figure 2 : Répartition de l'échantillon selon l’âge

L'âge moyen des patients était de 29,5 ans avec des âges variant de 19 ans à 44 ans.

5 patients étaient âgés de moins de 25 ans, 3 patients étaient âgés de 25 à 35 ans et 3 patients étaient âgés de plus de 35 ans. 


\subsubsection{Répartition de l'échantillon selon le niveau d'études}

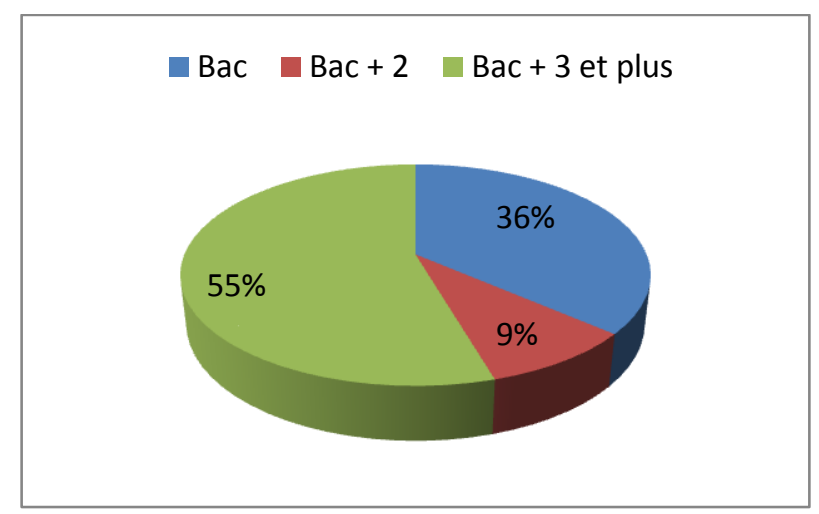

Figure 3 : Répartition de l'échantillon selon le niveau d'études

4 patients avaient un niveau bac, 1 patient avait un niveau bac +2 et 6 patients avaient un niveau bac +3 et plus.

Leurs professions respectives sont présentées dans le tableau 2.

\subsection{Caractéristiques médicales}

\subsubsection{Répartition de l'échantillon selon l'ancienneté du diabète}

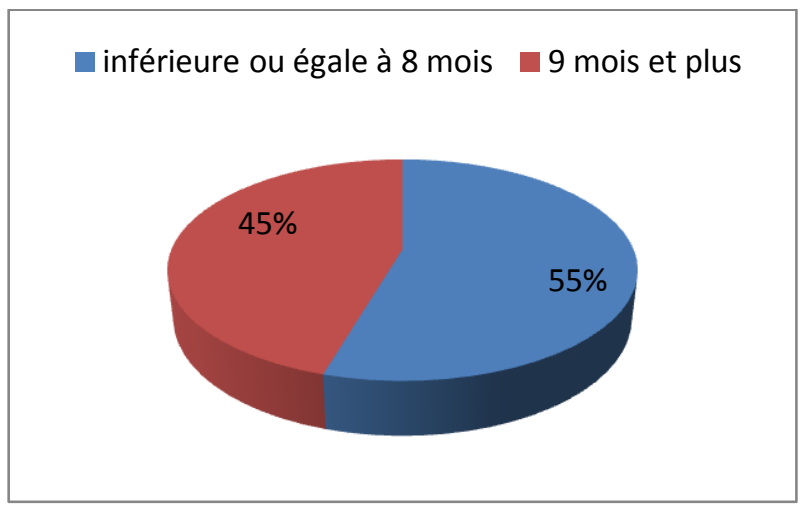

Figure 4 : Répartition de l'échantillon selon l'ancienneté du diabète

L'ancienneté de leur diabète était en moyenne de 10 mois. Elle variait de 2 mois à 35 mois. 6 patients étaient diagnostiqués depuis 8 mois ou moins et 5 patients depuis 9 mois et plus. 


\subsubsection{Répartition de l'échantillon selon le mode d'injection}

\section{d'insuline}

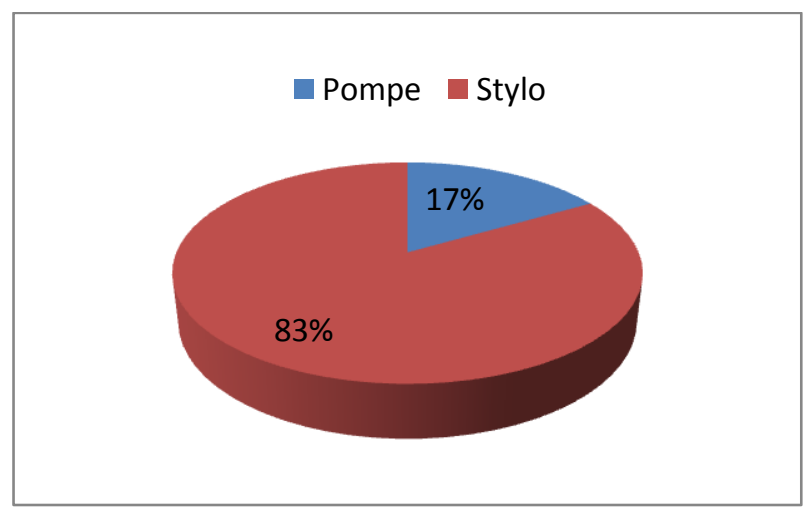

Figure 5 : Répartition de l'échantillon selon le mode d'injection d'insuline

1 patiente était sous pompe à insuline tandis que 10 patients faisaient leurs injections au stylo.

\subsubsection{Répartition de l'échantillon selon le statut vis-à-vis de l'ETP}

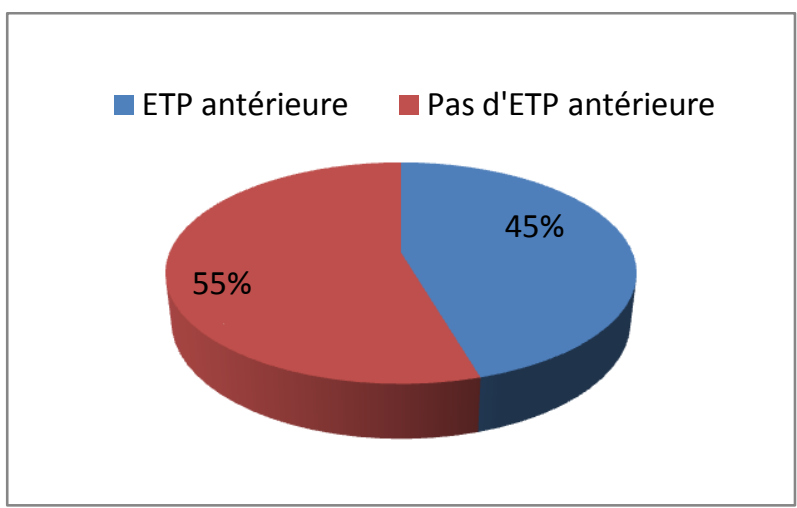

Figure 6 : Répartition de l'échantillon selon de statut vis-à-vis de l'ETP

Cinq patients avaient bénéficié d'une ETP antérieure entre 2011 et juin 2013. Six patients n'en avaient pas bénéficié.

Il est à noter que les connaissances acquises lors de l'hospitalisation initiale, d'hospitalisations ultérieures, en consultation ou au cours de recherches personnelles n'ont pas été prises en compte. Seuls les stages d'ETP effectués en structure hospitalière, durant un ou plusieurs jours, seul ou en groupe, ont été recensés. Ces données sont détaillées dans le tableau 2. 


\subsection{Les patients et Internet}

\subsubsection{L'accès des patients à Internet}

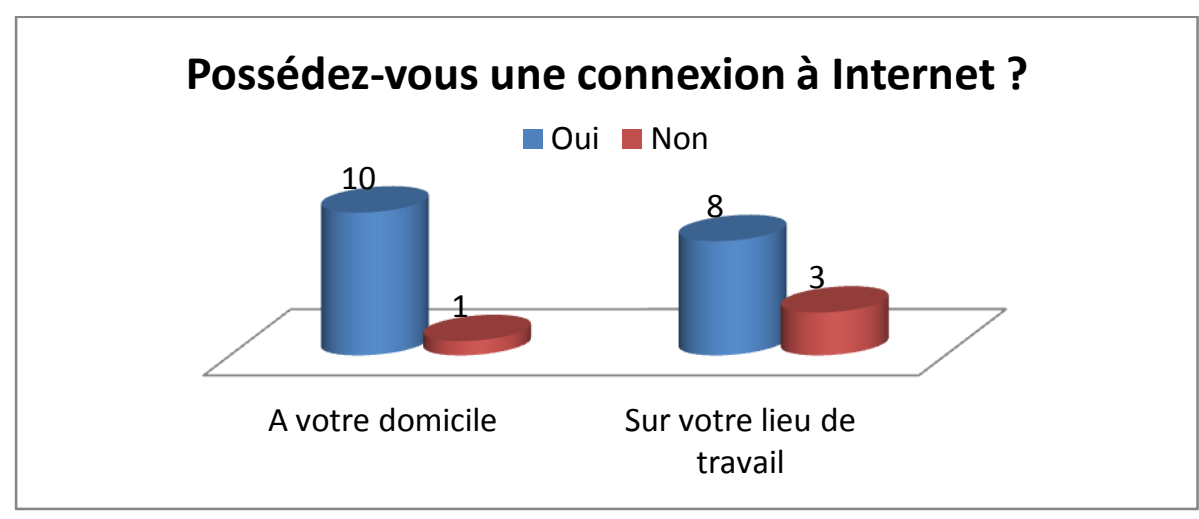

Figure 7 : Accès des patients à Internet

90,9\% des patients possédaient une connexion à Internet à leur domicile et 72,7\% des patients possédaient une connexion à Internet sur leur lieu de travail.

Le patient qui n'avait pas accès à Internet à son domicile possédait un accès sur son lieu de travail.

\subsubsection{Utilisation d'Internet par les patients}

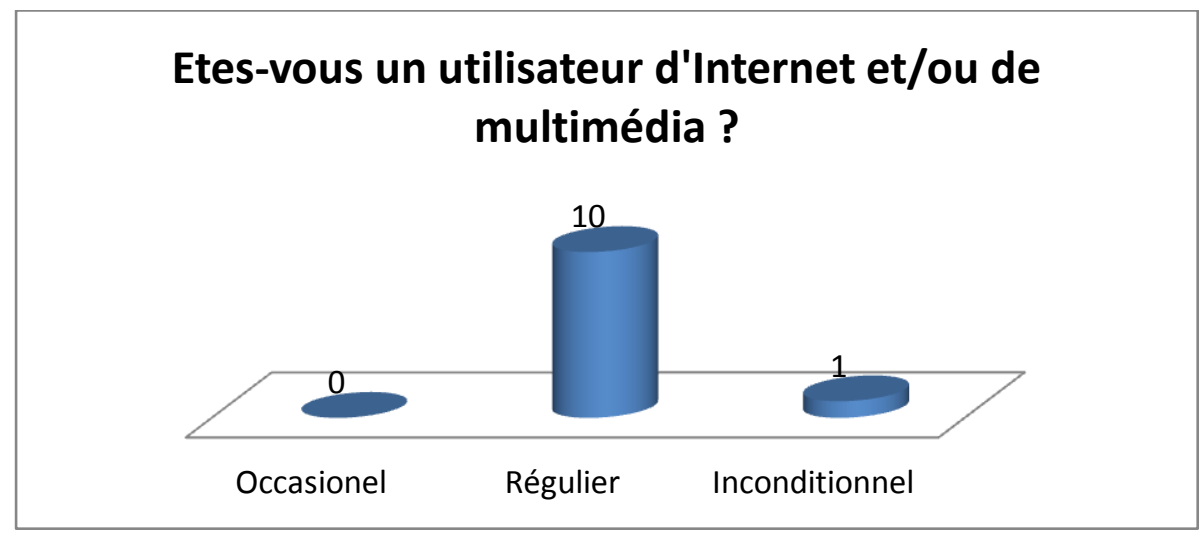

Figure 8 : Utilisation d'Internet et/ou de multimédia par les patients

90,9\% des patients se définissaient comme des utilisateurs réguliers d'Internet et/ou de multimédia et 1 patient $(9,1 \%)$ comme un utilisateur inconditionnel. 


\subsubsection{Recherches de santé sur Internet}

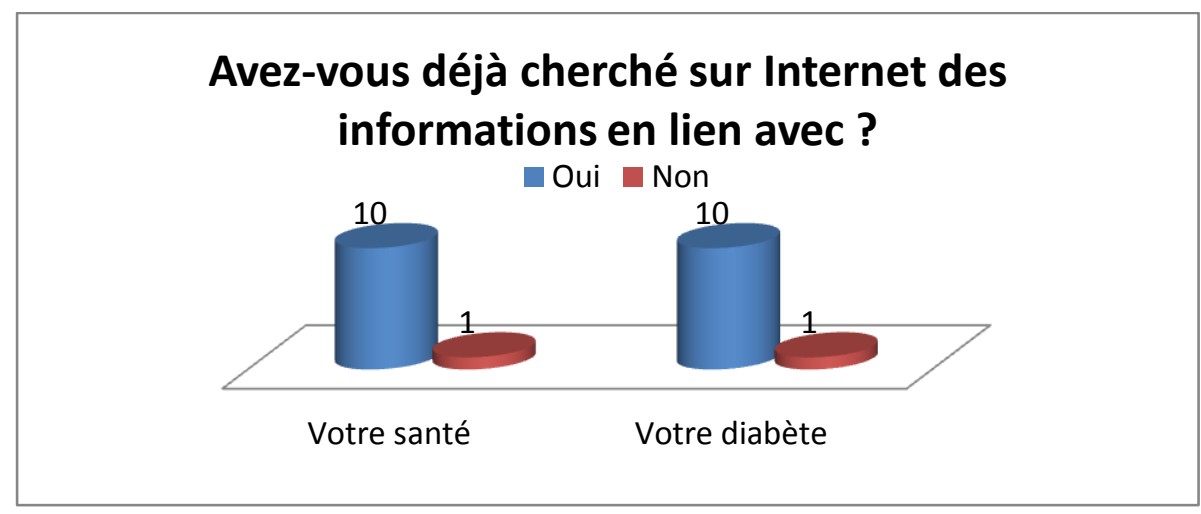

Figure 9 : Recherches de santé sur Internet

90,9\% des patients avaient déjà effectué des recherches en lien avec leur santé sur Internet et notamment sur le diabète de type 1 .

4 patients ont déclaré avoir fait des recherches sur les sites officiels des agences de santé et des agences du médicament, 4 sur des forums, 2 sur Doctissimo et 1 sur des sites d'associations de patients.

Seul 1 patient a déclaré n'avoir jamais cherché d'informations en lien avec sa santé et son diabète sur Internet. 


\section{Analyse du questionnaire dirigé et des entretiens semi-dirigés}

\subsection{Appréciations relatives à la qualité du contenu}

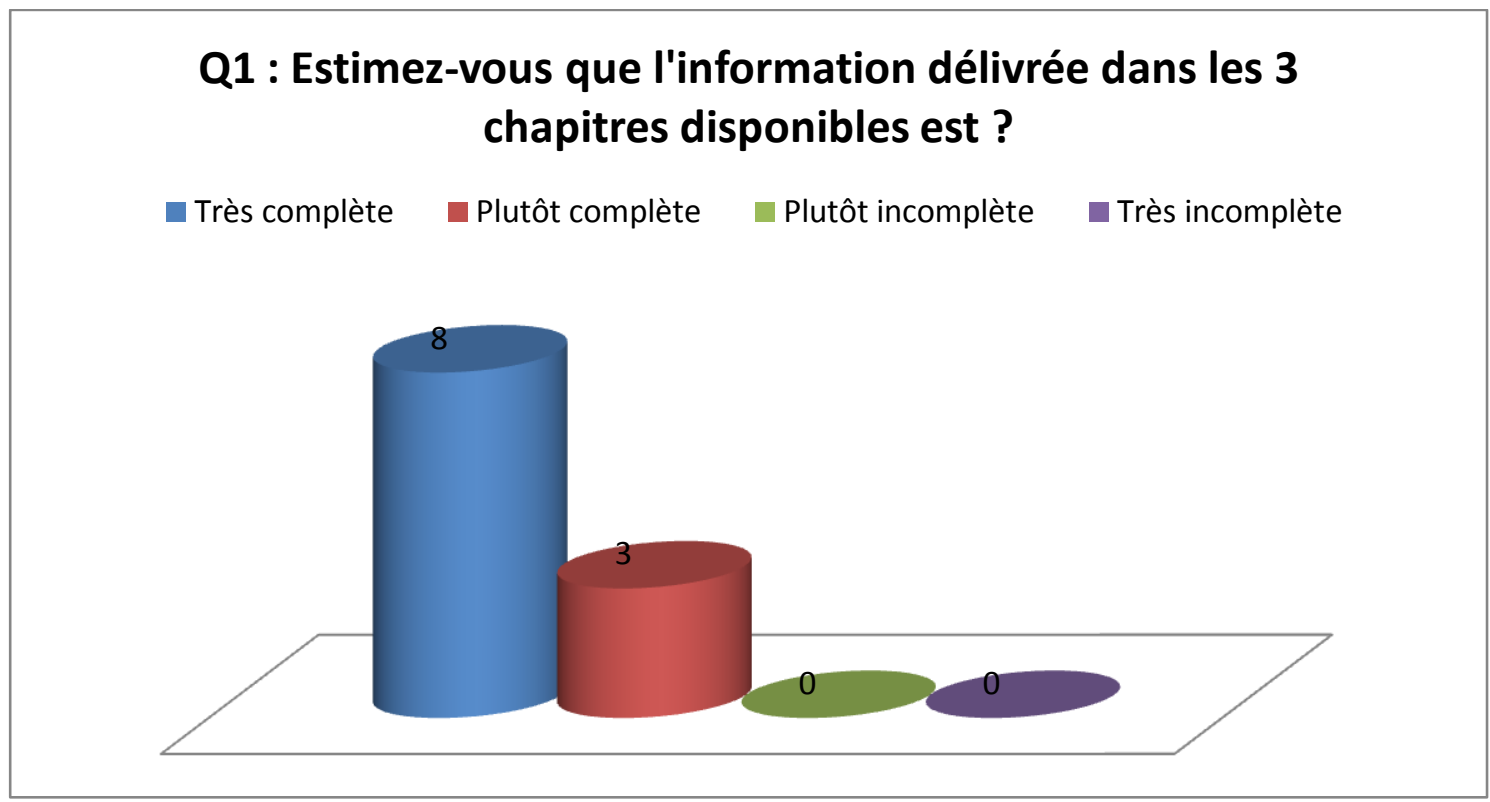

Figure 10 : Réponses à la question $\mathbf{n}^{\circ} 1$

L'ensemble des patients interrogés a considéré l'information délivrée dans le site INDIGO comme complète. $8(72,7 \%)$ ont pensé qu'elle était très complète et $3(27,3 \%)$ l'ont estimée plutôt complète.

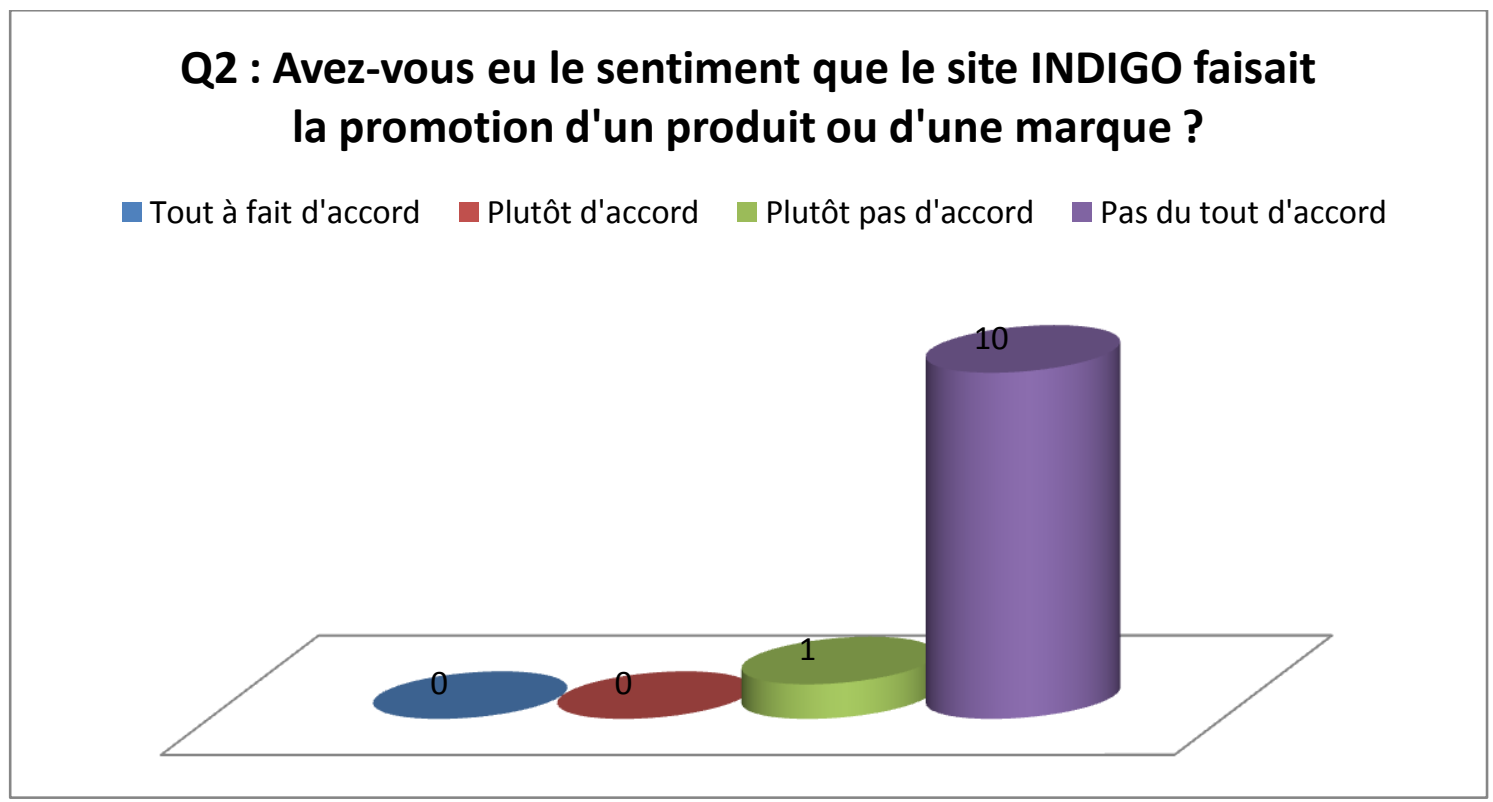

Figure 11 : Réponses à la question $\mathbf{n}^{\circ} 2$

L'ensemble des patients interrogés n'a pas eu le sentiment que le site INDIGO faisait la promotion d'un produit ou d'une marque. 1 patient $(9,1 \%)$ a déclaré n'être plutôt pas d'accord et 10 patients $(90,9 \%)$ ont déclaré n'être pas du tout d'accord. 


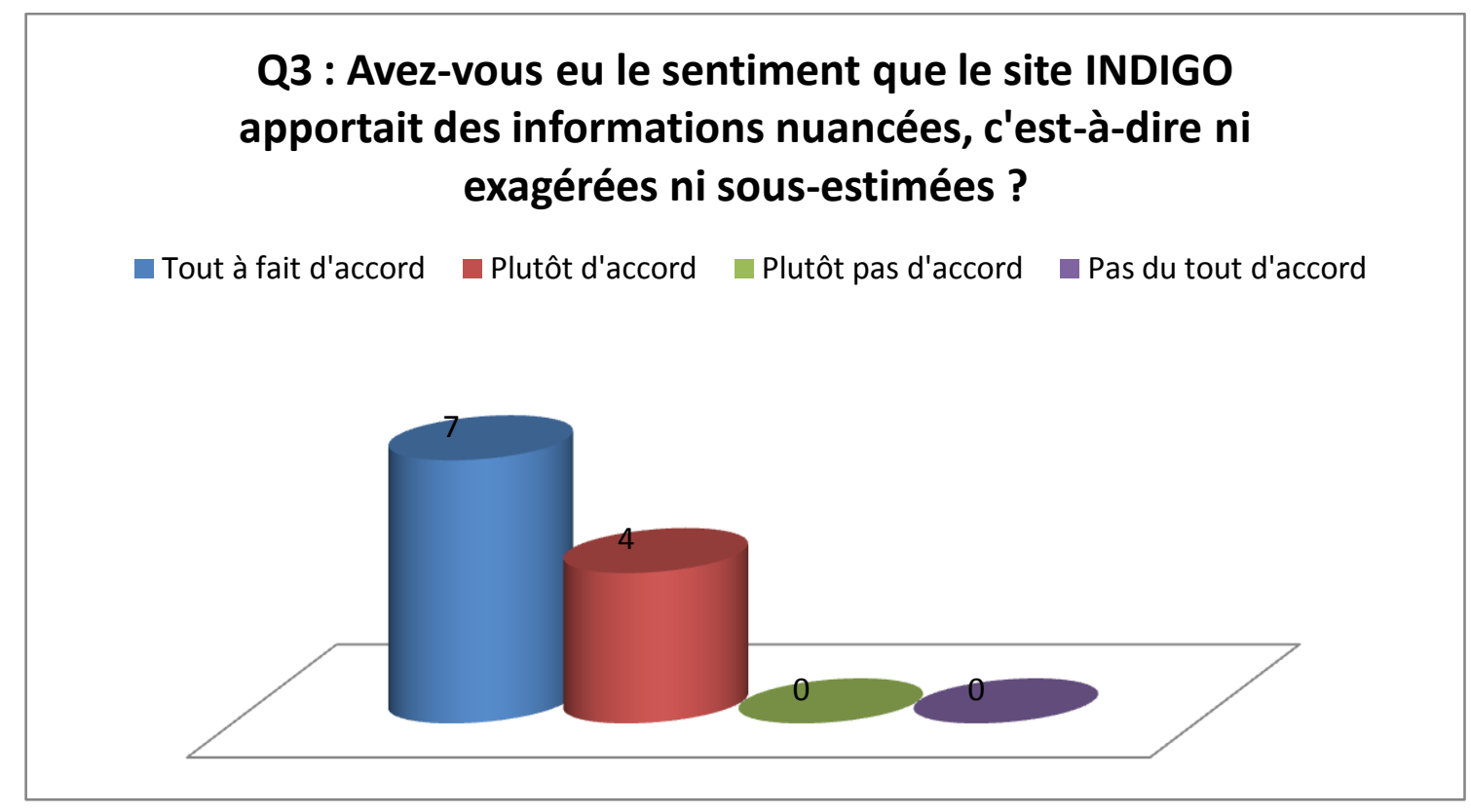

Figure 12 : Réponses à la question $\mathbf{n}^{\circ} 3$

Les patients interrogés ont estimé que le site INDIGO délivrait des informations nuancées. 7 patients $(63,6 \%)$ se sont dits tout à fait d'accord et 4 patients $(36,4 \%)$ se sont déclarés plutôt d'accord.

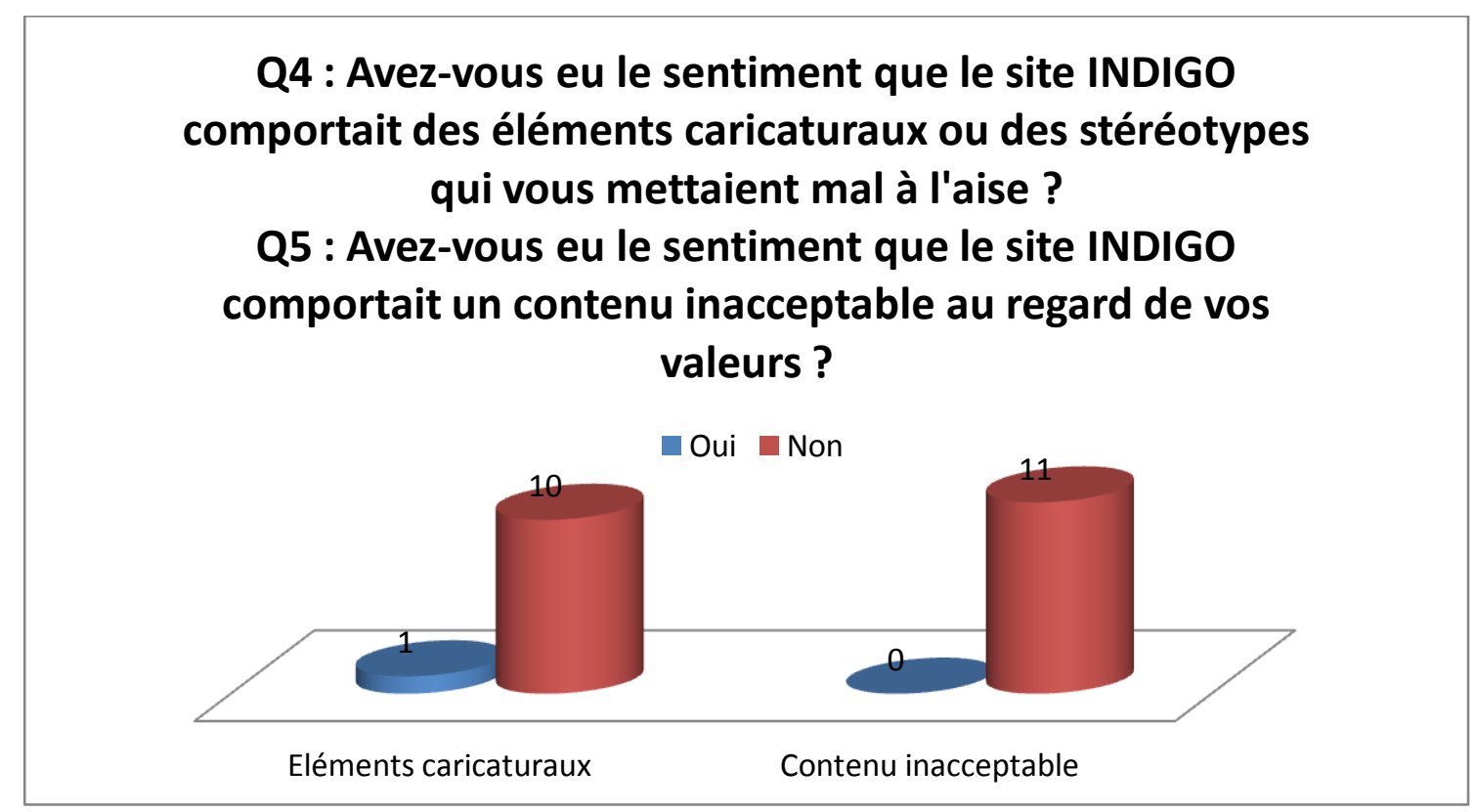

Figure 13 : Réponses aux questions $n^{\circ} 4$ et 5

1 patient $(9,1 \%)$ a déclaré avoir eu le sentiment que le site INDIGO comportait des éléments caricaturaux ou des stéréotypes qui l'avaient mis mal à l'aise. Nous n'avons pas de précision concernant sa déclaration. 
Aucun des patients interrogés n'a déclaré avoir eu le sentiment que le site INDIGO comportait un contenu inacceptable au regard de ses valeurs.

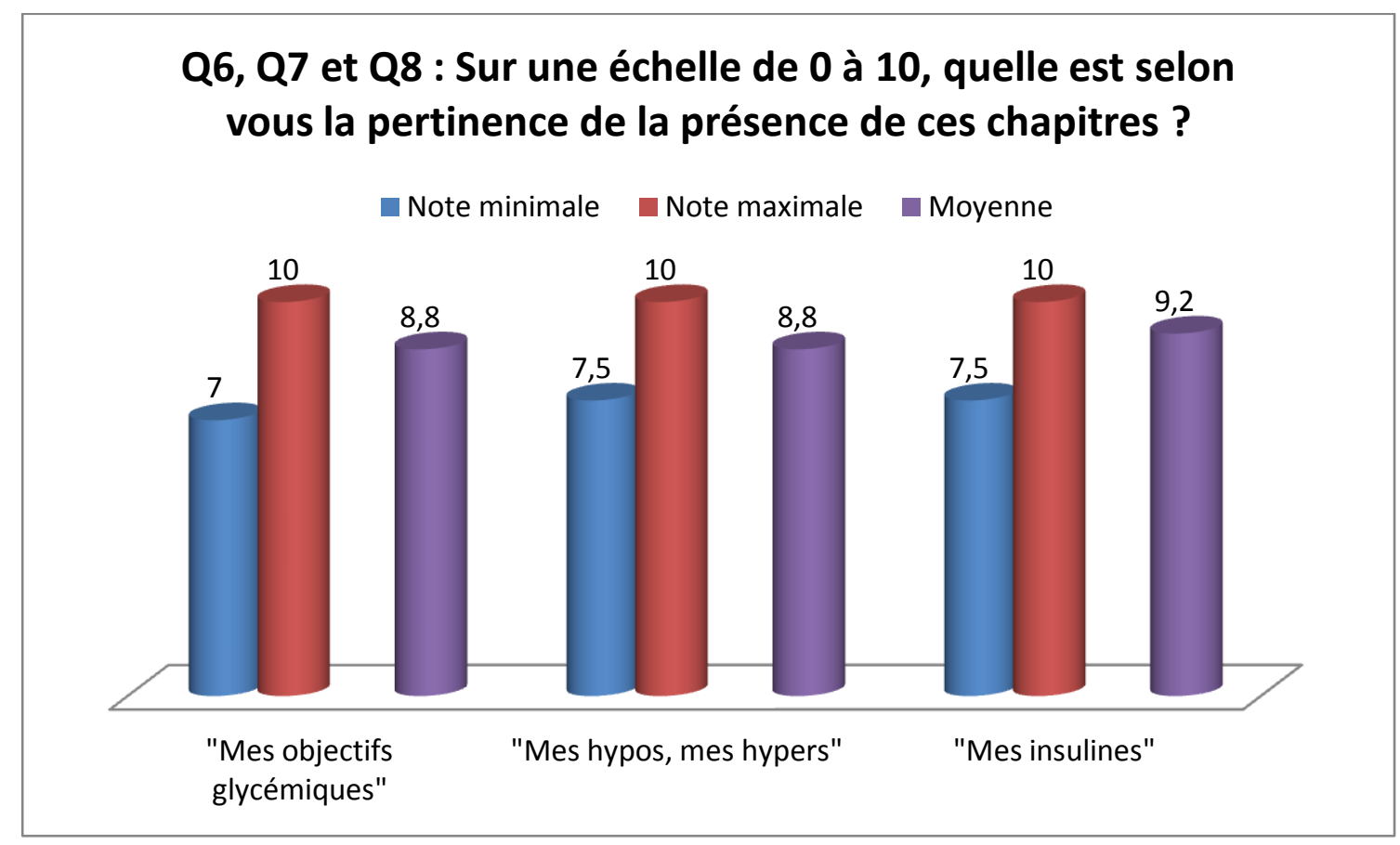

Figure 14 : Réponses aux questions $n^{\circ} 6,7$ et 8

Les patients interrogés ont estimé la présence du chapitre «Mes objectifs glycémiques » pertinente avec une note moyenne de 8,8/10. Les notes variaient de 7 à 10/10 (cf. Annexe 7).

Ils ont également trouvé pertinente la présence du chapitre «Mes hypos, mes hypers » avec une note moyenne de 8,8/10. Les notes variaient de 7,5 à 10/10 (cf. Annexe 7).

Il en était de même concernant la présence du chapitre «Mes insulines » avec une note moyenne de 9,2/10. Les notes variaient de 7,5 à 10/10 (cf. Annexe 7). 


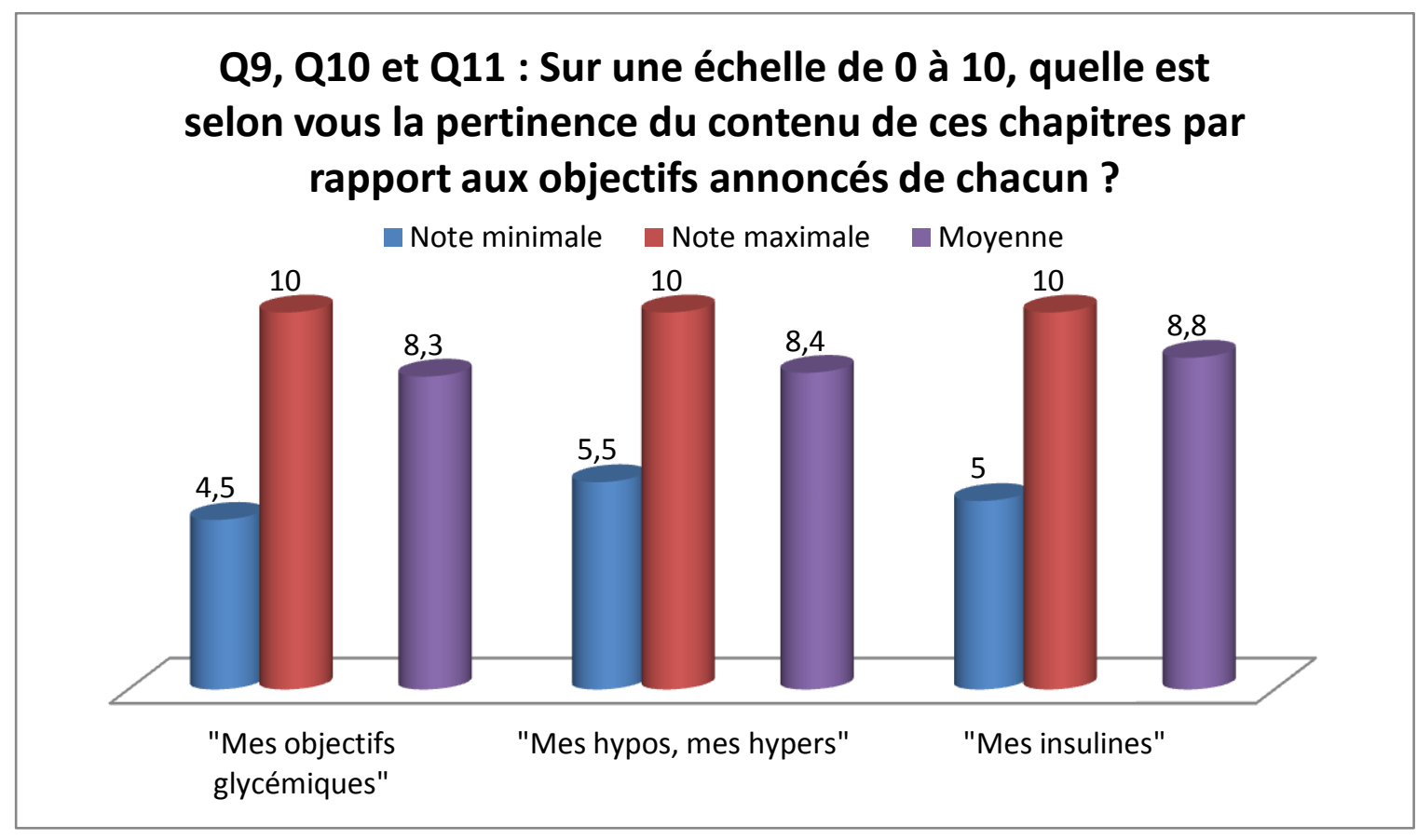

Figure 15 : Réponses aux questions $n^{\circ} 9,10$ et 11

Les patients interrogés ont noté la pertinence du contenu du chapitre «Mes objectifs glycémiques » par rapport aux objectifs annoncés (définir une plage de glycémie «acceptable », analyser où vous en êtes par rapport à cette plage et déterminer vos objectifs de réduction de fréquence de vos hypoglycémies et de vos hyperglycémies) à 8,3/10 en moyenne. Les notes variaient de 4,5 à 10/10. La note de 4,5/10 a été attribuée par la patiente $n^{\circ} 6$. Le patient $n^{\circ} 3$ a attribué la note de 5/10. Les autres notes étaient supérieures à 7/10 (cf. Annexe 7).

La pertinence du contenu du chapitre « Mes hypos, mes hypers » par rapport aux objectifs annoncés (gérer les fluctuations de votre glycémie afin que celles-ci ne vous empêchent de vivre pleinement) a été notée à $\mathbf{8 , 4 / 1 0}$ en moyenne. Les notes variaient de 5,5 à 10/10. La note de 5,5/10 a été attribuée par la patiente $n^{\circ} 6$. Les autres notes étaient supérieures à 7/10 (cf. Annexe 7).

La pertinence du contenu du chapitre «Mes insulines » par rapport aux objectifs annoncés (comprendre ce qu'est l'insuline, son fonctionnement afin de mieux équilibrer vos glycémies au quotidien) a été notée à 8,8/10 en moyenne. Les notes variaient de 5 à 10/10. La note de $5 / 10$ a été attribuée par la patiente $n^{\circ} 6$. Les autres notes étaient supérieures à 8/10 (cf. Annexe 7).

Deux patients n'ont pas répondu à cette question puisqu'ils n'avaient pas vu ce chapitre. 
N.B. : Les objectifs des différents chapitres étaient rappelés dans l'énoncé de chaque question (cf. questionnaire de l'entretien dirigé en Annexe 5)

$\mathrm{Au}$ total, les patients interrogés ont considéré que le contenu du site INDIGO était en adéquation avec les critères de qualité d'un outil d'intervention en éducation pour la santé de l'INPES.$~{ }^{28}$ Ils ont estimé que le site était complet, sans caractère promotionnel et qu'il délivrait des informations nuancées. Les patients dans une large majorité $(90,9 \%)$ ne se sont pas sentis mis mal à l'aise par des éléments caricaturaux ou des stéréotypes. Ils n'ont pas signalé de contenu inacceptable au regard de leurs valeurs. Ils ont jugé les thèmes abordés par les chapitres pertinents. Ils ont tous attribué une note supérieure ou égale à 5/10 à l'adéquation du contenu des chapitres «Mes hypos, mes hypers » et « Mes insulines » à leurs objectifs annoncés et 90,9\% des patients ont attribué une note supérieure ou égale à 5/10 à l'adéquation du contenu du chapitre « Mes objectifs glycémiques » à ses objectifs annoncés.

L'analyse des entretiens semi-dirigés nous en a appris davantage sur les appréciations des patients interrogés concernant le contenu du site INDIGO.

Les citations des patients sont présentées entre guillemets et en italique. Le chiffre entre parenthèses correspond au numéro attribué à chaque patient.

Ils ont trouvé le site INDIGO clair et complet : «c'était bien, complet» $(1)$; «c'est complet comme ça» (4); "j'ai beaucoup apprécié déjà parce que c'est clair, c'est complet » $(5)$; «c'est très bien parce que ça explique tout le diabète en fait » (11).

Ils ont estimé que les situations essentielles étaient abordées : "c'est très bien, ça retrace bien l'ensemble du diabète et ça explique vraiment bien ce qu'il en est » (11); "je pense qu'il y a un peu toutes les situations, il y a l'essentiel qui est dit, c'est très bien » (7).

Les informations ont été jugées utiles : "il m'a donné des infos que je n'avais pas, c'était bien utile, bien intéressant d'avoir cette information de plus» $(4)$; " c'est toujours utile d'en découvrir un peu plus» (1).

Les patients ont signalé l'intérêt de trouver une information fiable et de qualité sur Internet, en laquelle ils pouvaient avoir confiance : "avec Internet c'est un peu dur de 
savoir ce qui est juste, ce qui est faux, là au moins on sait que ça a été recommandé par mon diabétologue » (2) ; "je l'ai trouvé intéressant et des sites comme ça il ne doit pas y en avoir beaucoup sur Internet, quand on tape diabète on tombe sur des forums mais il n'y pas beaucoup de sites comme ça interactifs et qui se penchent sur le diabète de type 1 en particulier $\gg(9)$.

Ils ont souligné le fait que cette information n'ait pas été source d'inquiétude ni d'angoisse pour eux : «le site dans tous les cas ne m'a pas donné d'inquiétude ça c'est sûr » (4) ; «ce n'est pas un site qui est anxiogène, jamais, ça ne fait pas peur du tout » (7). Une patiente l'a comparé à ce qu'elle pouvait trouver d'autre sur Internet : «les autres sites j'ai peu fait j'avoue parce que je me méfie, surtout quand il s'agit de la maladie, ça fait vite peur parce qu'il y a toutes sortes de situations, on a tellement tout et rien sur Internet, c'est tellement compliqué, c'est tellement justement anxiogène avec les forums... J'ai vite abandonné, je n'aime pas trop ça les forums, je trouve que ce n'est pas forcément adapté »(7).

Certains patients se sont sentis rassurés par les informations contenues dans le site INDIGO : «le site m'a rassurée, ça sérénise ; c'était un petit soulagement » (1); " c'est très important de nous rassurer parce que pour avoir discuté avec d'autres diabétiques de type 1 on est quand même très stressés par les séquelles éventuelles » (7) ; «le chapitre 1 m'a un peu rassurée » (9) ; "la séquence sur le Glucagon ça m'a rassurée quand même, c'est plus parlant de le voir» (10) ; "le chapitre 1 ça rassure un petit peu de voir ça» (11).

Une patiente a souligné le fait que l'information n'était pas caricaturale : «il y a une question sur si ça nous met mal à l'aise, et bien pas du tout, j'ai trouvé que ce n'est pas dans le cliché » (7). Elle a également noté le caractère nuancé de l'information : «en même temps les choses sont montrées telles qu'elles sont » (7).

Un patient a évoqué le fait que le site INDIGO n'était pas exhaustif mais a précisé pourquoi il ne pouvait pas l'être : «bien sûr on ne peut pas donner tous les cas pour tout le monde, c'est difficile d'ajouter des choses. Le protocole de soins il faut le regarder au cas par cas, je ne pense pas que l'on puisse mettre plus d'infos, aller plus dans le détail, à la fin ça devient limite dangereux. Je ne pense pas que le but c'est de remplacer le diabétologue »(4). 
3.2.Appréciations relatives à la qualité pédagogique

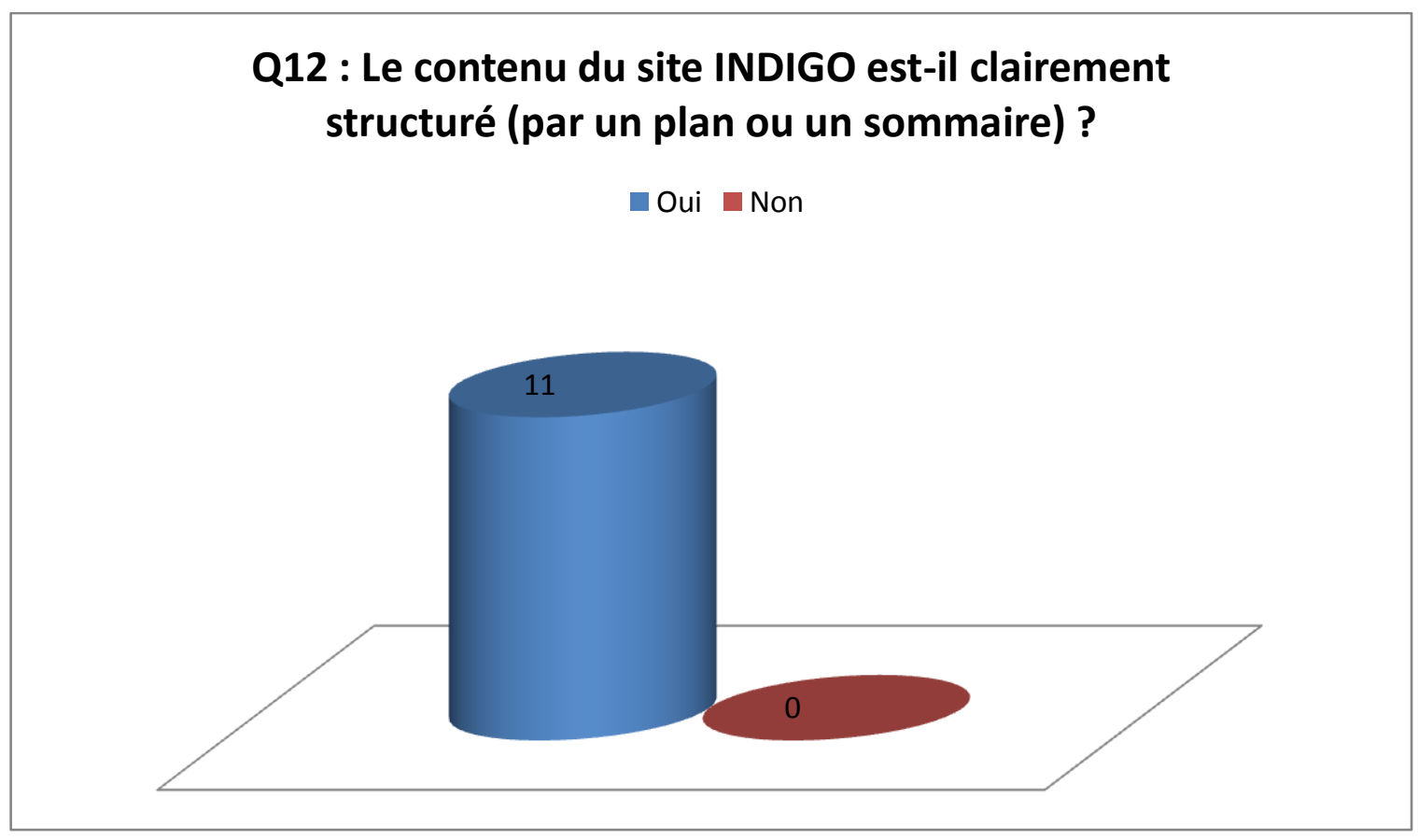

Figure 16 : Réponses à la question $n^{\circ} 12$

La totalité des patients interrogés a constaté que le site INDIGO était clairement structuré par un plan ou un sommaire.

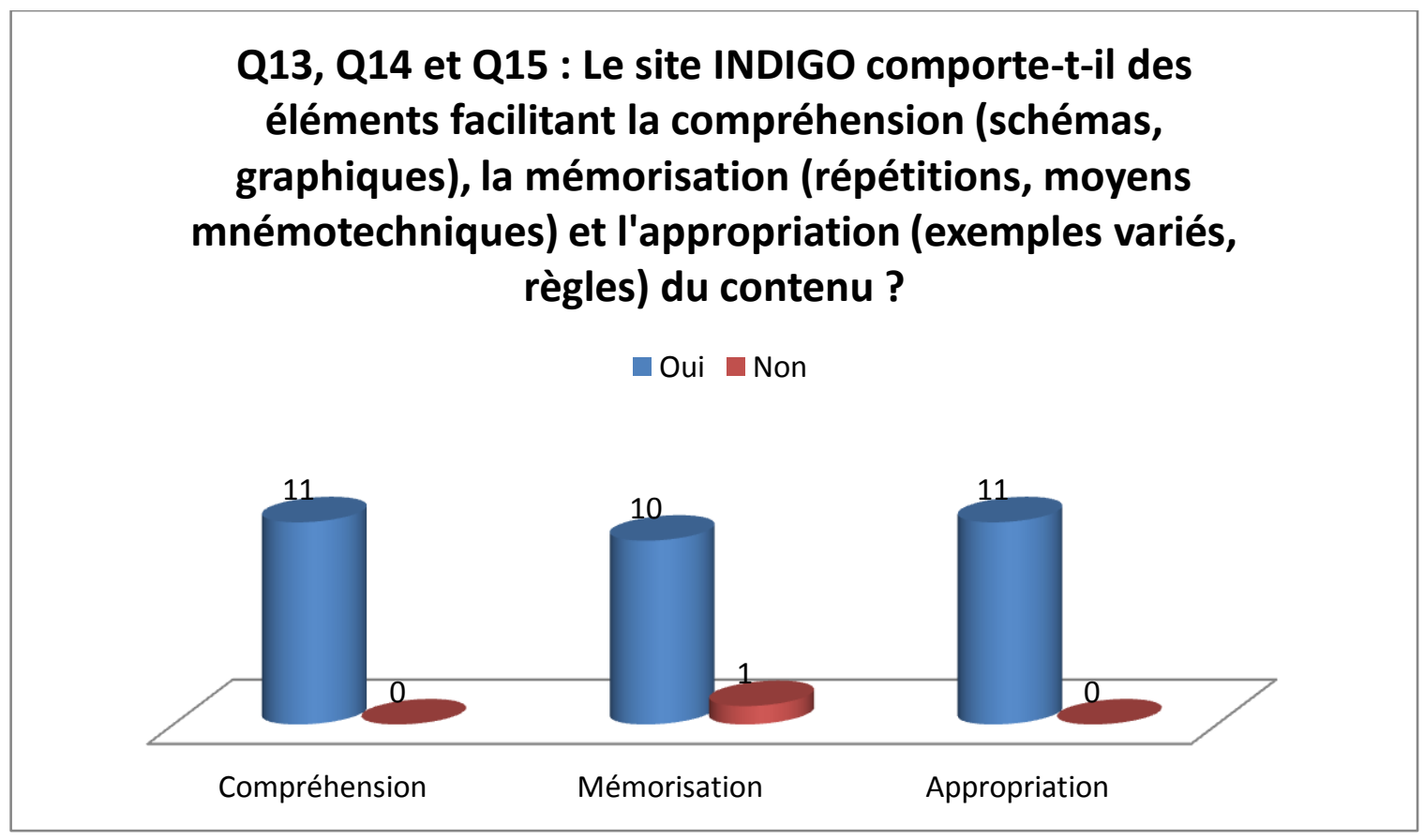

Figure 17 : Réponses aux questions $n^{\circ} 13,14$ et 15

L'ensemble des patients a estimé que le site INDIGO comportait des éléments facilitant la compréhension, tels que des graphiques et des schémas, ainsi que des éléments facilitant l'appropriation du contenu, tels que des exemples variés et des règles. 
10 patients $(90,9 \%)$ ont considéré qu'il comportait également des éléments facilitant la mémorisation, tels que des répétitions et des moyens mnémotechniques.

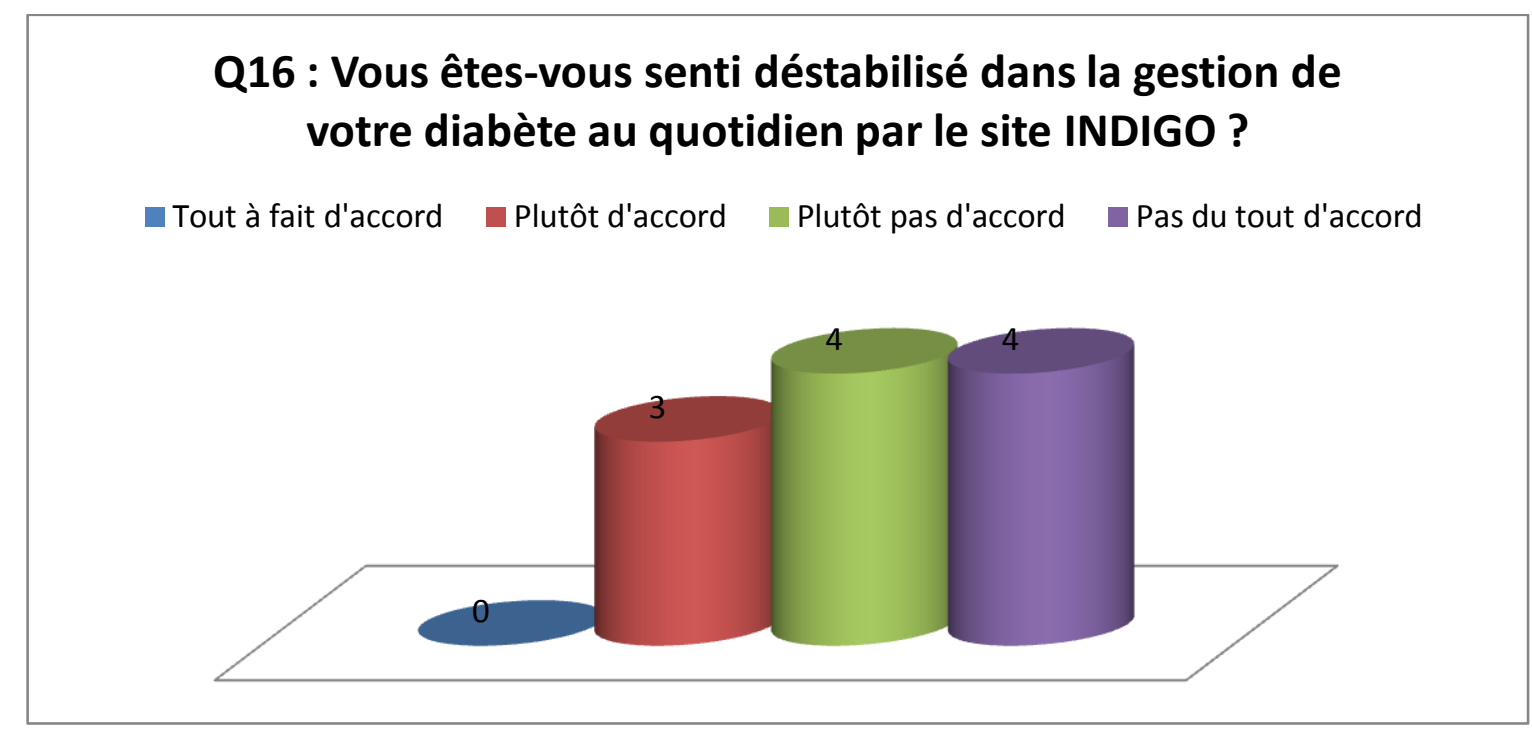

Figure 18 : Réponses à la question $n^{\circ} 16$

3 patients $(27,2 \%)$ ont déclaré s'être sentis plutôt déstabilisés par le site INDIGO dans la gestion de leur diabète au quotidien. 4 patients $(36,4 \%)$ ont répondu qu'ils n'étaient plutôt pas d'accord et 4 patients $(36,4 \%)$ qu'ils n'étaient pas du tout d'accord.

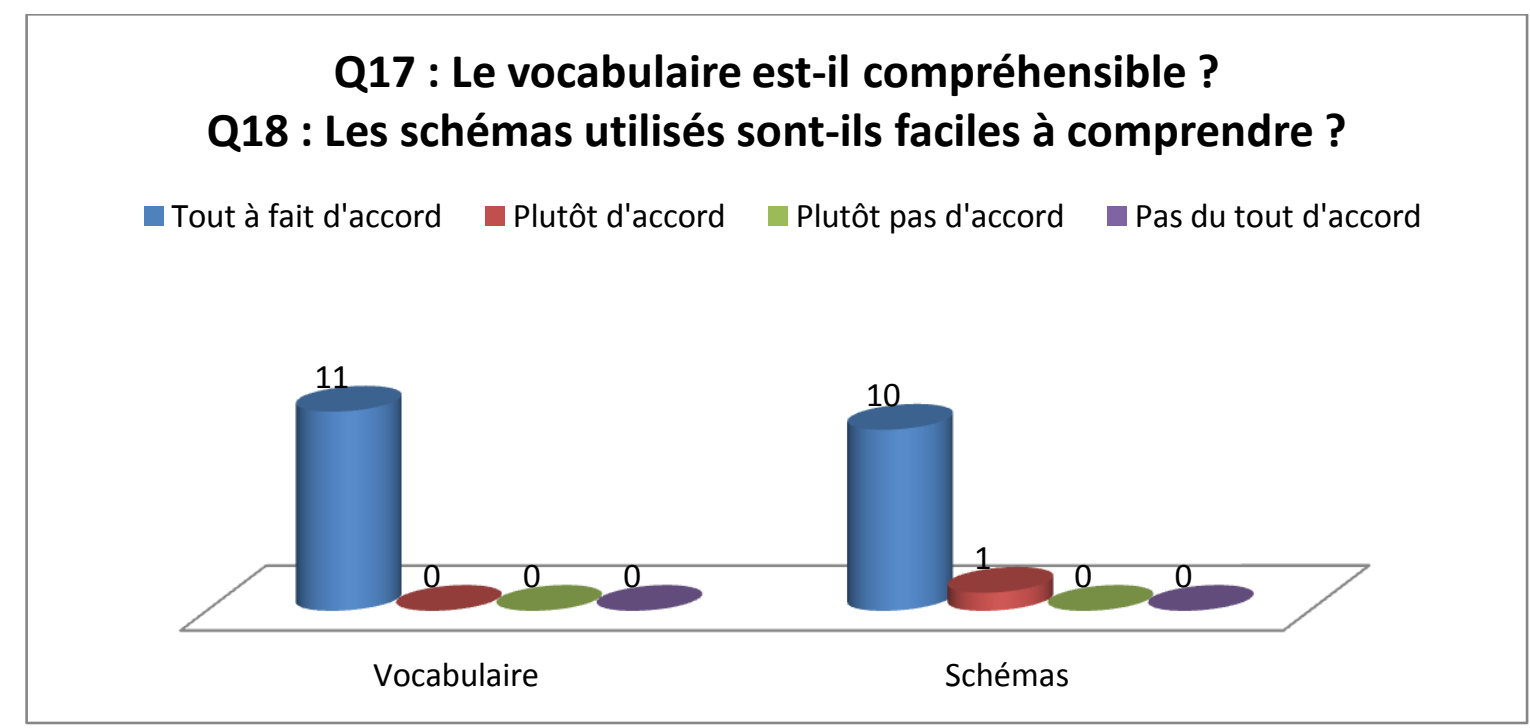

Figure 19 : Réponses aux questions $n^{\circ} 17$ et 18

L'ensemble des patients a estimé que le vocabulaire employé dans le site INDIGO était compréhensible. 10 patients $(90,9 \%)$ étaient tout à fait d'accord pour dire que les schémas utilisés étaient faciles à comprendre et 1 patient $(9,1 \%)$ était plutôt d'accord. 


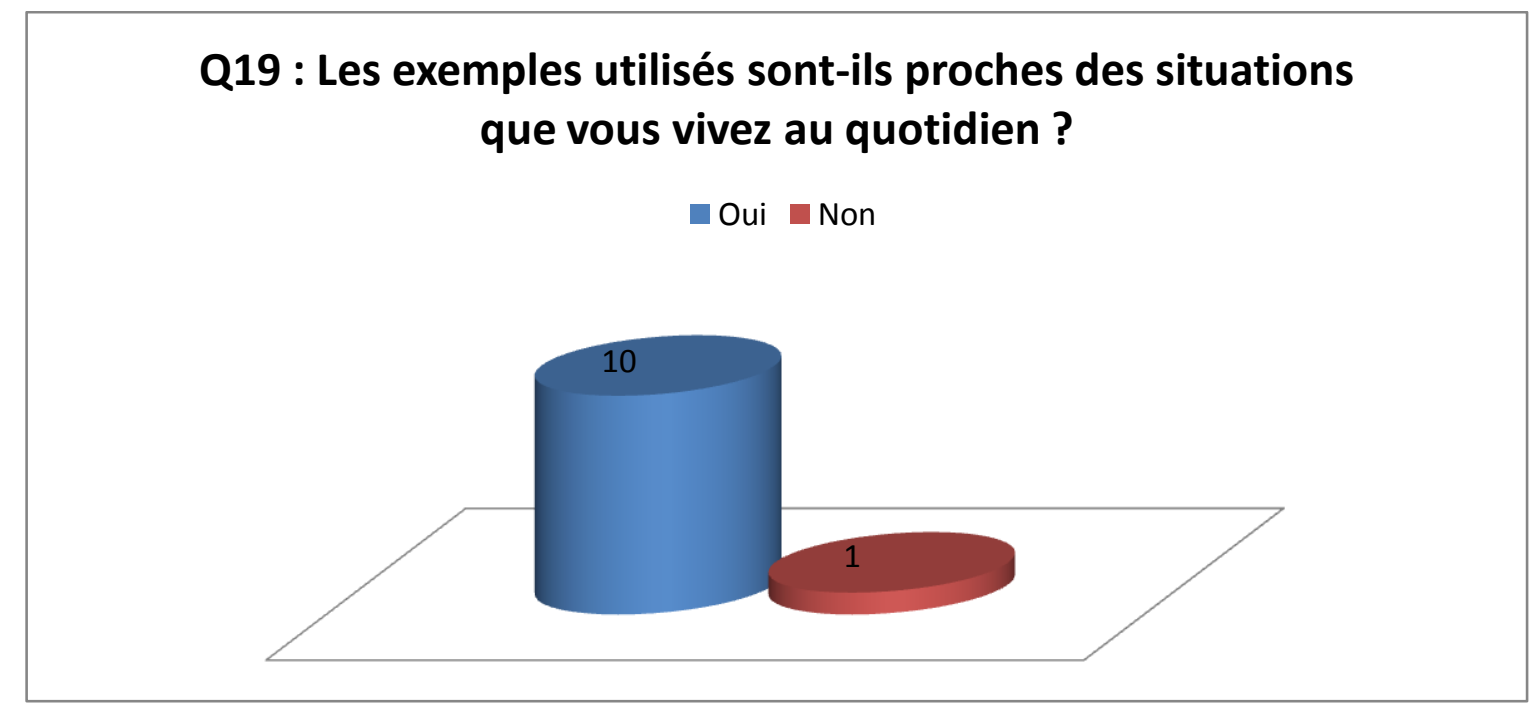

Figure 20 : Réponses à la question $n^{\circ} 19$

10 patients $(90,9 \%)$ ont affirmé que les exemples utilisés étaient proches des situations qu'ils vivaient au quotidien.

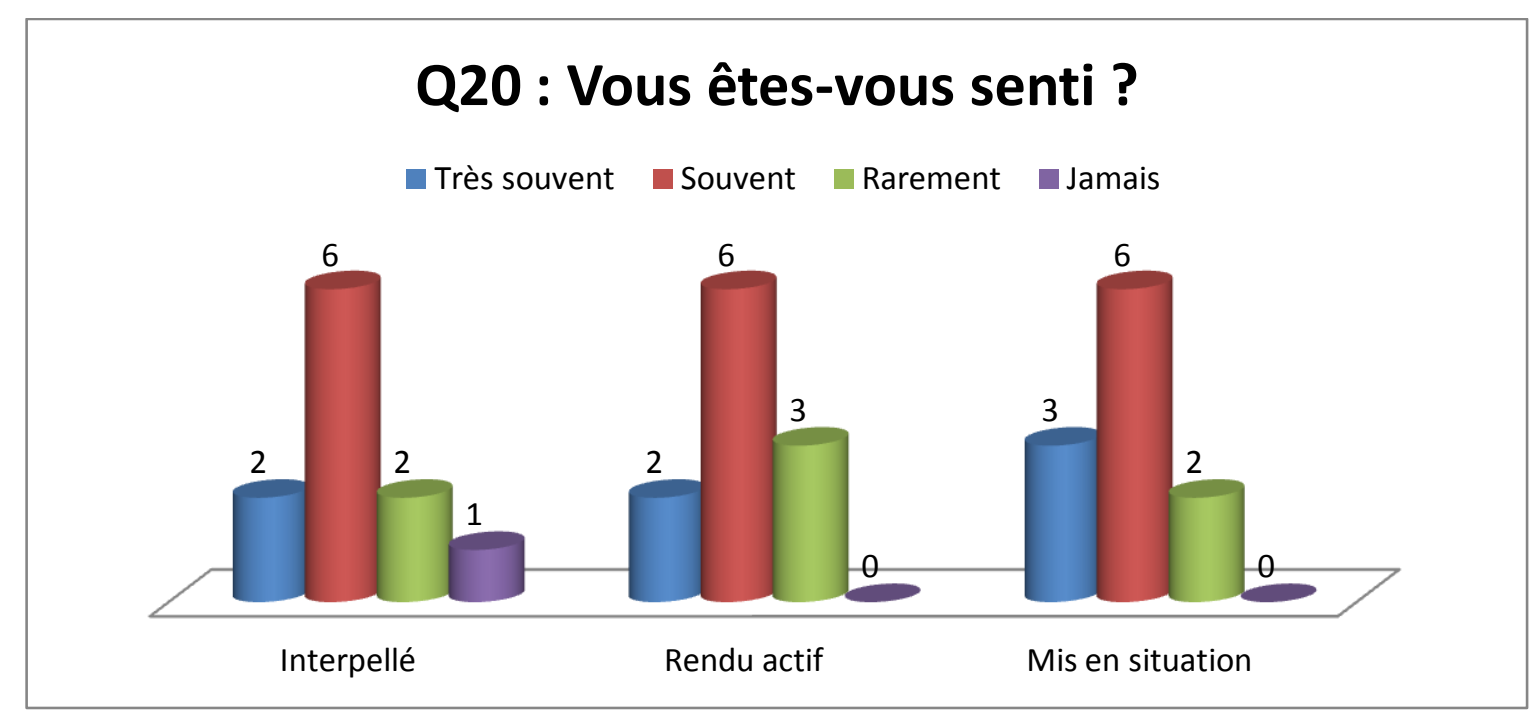

Figure 21 : Réponses à la question $\mathbf{n}^{\circ} 20$

2 patients $(18,2 \%)$ se sont sentis très souvent interpellés, $6(54,5 \%)$ souvent, $2(18,2 \%)$ rarement et $1(9,1 \%)$ jamais.

2 patients $(18,2 \%)$ se sont sentis très souvent rendus actifs, $6(54,5 \%)$ souvent et $3(27,3 \%)$ rarement.

3 patients $(27,3 \%)$ se sont très souvent sentis mis en situation, $6(54,5 \%)$ souvent et 2 $(18,2 \%)$ rarement. 


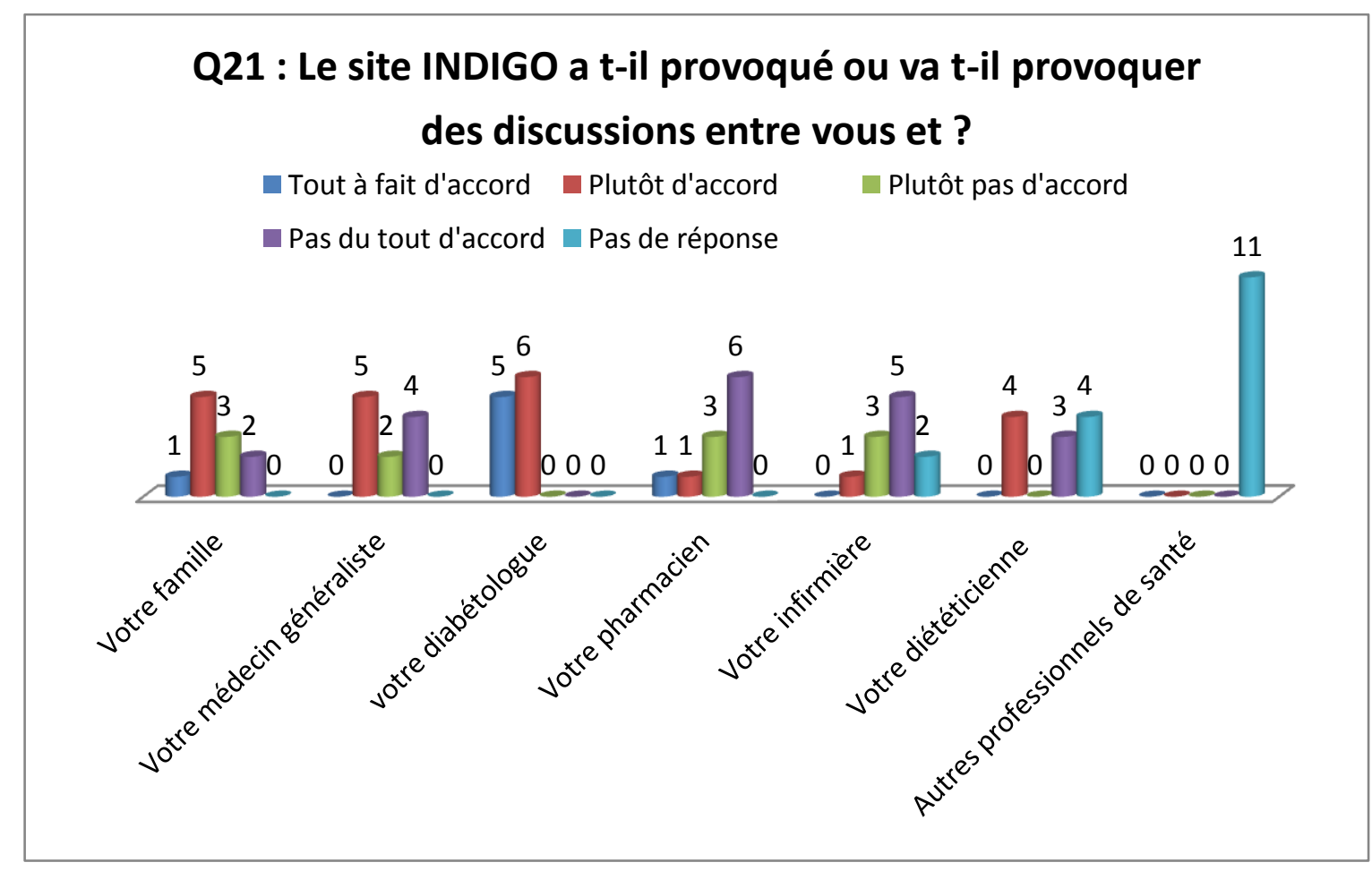

Figure 22 : Réponses à la question $\mathbf{n}^{\circ} 21$

6 patients $(54,5 \%)$ ont envisagé de parler du site INDIGO avec un membre de leur entourage.

5 patients $(45,4 \%)$ ont déclaré qu'ils souhaitaient en discuter avec leur médecin généraliste et tous ont envisagé d'en discuter avec leur diabétologue.

2 patients $(18,2 \%)$ ont envisagé d'en discuter avec leur pharmacien, 1 patient $(9,1 \%)$ avec son infirmière et 4 patients $(36,4 \%)$ avec leur diététicienne.

Au total, les patients interrogés ont considéré que le site INDIGO possédait une qualité pédagogique en adéquation avec les critères de qualité d'un outil d'intervention en éducation pour la santé de l'INPES. ${ }^{28}$ Ils ont estimé que le site était clairement structuré, qu'il comportait des éléments facilitant la compréhension et l'appropriation du contenu par l'utilisateur. 90,9\% ont jugé qu'il possédait des éléments facilitant la mémorisation. Pour eux, le vocabulaire employé était compréhensible et ceci même pour le patient $n^{\circ} 4$ qui est d'origine italienne et qui parle le français depuis 3 ans seulement. Tous ont estimé que les schémas utilisés étaient faciles à comprendre. 90,9\% ont jugé que les exemples proposés étaient proches des situations qu'ils vivaient au quotidien.

La majorité des patients $(72,7 \%)$ ne s'est pas sentie déstabilisée dans la gestion de son diabète au quotidien par le site INDIGO. $72,7 \%$ des patients interrogés se sont sentis 
interpellés très souvent ou souvent. $72,7 \%$ se sont sentis rendus actifs très souvent ou souvent et $81,8 \%$ se sont sentis mis en situation très souvent ou souvent.

Enfin les patients interrogés ont déclaré que le site INDIGO allait être un sujet de discussion avec leur entourage pour 54,5\% d'entre eux, avec leur diabétologue pour $100 \%$ d'entre eux et avec leur médecin traitant pour $45,4 \%$ d'entre eux.

L'analyse des entretiens semi-dirigés nous a donné plus de détails sur les appréciations des patients interrogés concernant la qualité pédagogique du site INDIGO.

Les patients interrogés ont apprécié la structure du site : «le site est bien structuré, c'est vraiment très clair » (7) ; «au niveau du menu je trouve que c'est bien agencé, cette forme un peu circulaire, c'est clair »(9).

D'abord parce qu'elle leur a permis de s'orienter facilement : «avec le sommaire, enfin le plan on sait où on va » (2) ; "c'est facile d'avoir accès aux différents chapitres » (3).

Ensuite parce qu'elle leur a permis de choisir une séquence spécifique : «vu que c'est bien structuré on peut aller directement quelque part, on n'est pas obligé de se coltiner tous les chapitres pour aller à celui qui nous intéresse » (2) ; «on peut directement aller chercher une réponse à une question que l'on se pose au bon endroit » (7) ou de revenir facilement à un endroit particulier : "si on a un petit doute à un endroit on peut facilement y accéder à nouveau» (2); «c'est un outil sur lequel on revient d'où l'importance d'être très clair dans les chapitres »(7).

En ce qui concerne l'organisation des chapitres, certains l'ont trouvée logique et progressive: «les chapitres je ne vois pas dans quel autre ordre ils auraient pu apparaitre, je trouve que c'est logique» (9); "j'ai trouvé que le chapitre 1 était une bonne introduction à la maladie dans le sens qu'est-ce qu'on doit faire, comment se soigner. Les deux autres chapitres vont beaucoup plus dans le détail. Je l'ai trouvé bien et je pense qu'il faut avoir un chapitre comme ça pour ouvrir toutes les autres discussions. C'est un bon démarrage pour rentrer dans le monde du diabète » (4) ; "au niveau de ce qu'il dit, le chapitre 1, je trouve qu'il est bien à la première place. C'est-à-dire qu'il est introductif, assez global. Il est un peu moins compliqué que les autres. Enfin je veux dire c'est progressif je trouve » (5).

Cependant deux patients ont proposé d'inverser l'ordre du chapitre 2 « Mes hypos, mes hypers » et du chapitre 3 «Mes insulines » : «peut-être que le chapitre 2 et le chapitre 3 
on pourrait les inverser. Après avoir donné les objectifs, dire comment on peut arriver à cet objectif donc à travers l'insuline. Et comme $3^{\text {ème }}$ chapitre dire ce qui va arriver: qu'est-ce que l'hypo et l'hyper. Parfois je pensais ça. Par contre c'est aussi vrai que c'est difficile de dire mes objectifs de glycémie c'est un certain chiffre et de ne pas donner les informations pour dire ce qui arrive si on n'est pas là, donc les hypos et les hypers donc... (4); en fait comme les hypos et les hypers c'est un peu le résultat de ce que l'on fait avec l'insuline, je me disais que mettre « Mes insulines » avant « Mes objectifs glycémiques »... Mais bon après ça risque de compliquer un peu. Là comme ça, c'est vrai que c'était clair. Mais c'est vrai que je vois d'abord l'insuline et ensuite le résultat qui vient après » (5).

Ils ont estimé que les explications étaient claires : "c'est très bien expliqué, ça va à l'essentiel » $(1)$; "je trouve que c'est très intéressant, très utile de donner des explications faciles à comprendre »(4), "c'est très intéressant quand on a des interrogations, ça permet de répondre à des questions de façon très claire, très précise " (7) ; " c'est très pédagogique, il y a même un côté scolaire » (7).

Certains ont souligné le fait que le rythme était adapté : «le fond, le contenu était bien, c'était bien expliqué, clairement, étape par étape, ça n'allait pas trop vite sur le dosage d'informations » (2); «c'est un site qui est très facile d'accès, très pédagogique, par les témoignages, par le rythme, par les petits schémas colorés etc. » (7).

Parmi les outils pédagogiques utilisés, les schémas ont été les plus commentés.

Ils ont été appréciés par les patients interrogés : «j'ai bien aimé les explications avec les schémas, les courbes" (7); "j'ai aimé les récapitulatifs, les schémas à la fin d'une explication » (9) ; «les schémas j'ai trouvé que c'était très bien » (11).

Ils ont été considérés comme facilitant la compréhension : "c'est simple à comprendre, il y a des schémas, des illustrations. Les schémas j'ai trouvé ça clair » (5) ; "je suis très visuelle, du coup je trouve que les schémas c'était très bien » $(11)$; "les graphiques que l'on voit se faire ça aide à la compréhension, le fait que ce soit étape par étape » (3).

Un schéma a été fréquemment cité par les patients. Il s'agit du schéma utilisé dans le chapitre 2.4 : «Je veux aller plus loin dans l'analyse de l'estimation de ma glycémie » et qui correspond à la grille d'analyse des erreurs (cf. Annexe 8).

Certains patients ont déclaré n'avoir eu aucune difficulté à le comprendre : «je suis en école d'ingénieur donc la forme c'était bien compréhensible, mais pour ma copine aussi » 
(2). Pour d'autres cela a nécessité un peu plus de temps et de réflexion : " il m'a fallu un petit moment pour capter toutes les nuances » (1) ; "c'est vrai que ça fait un peu peur toutes ces couleurs et tout, au début on ne comprend pas tout à fait mais après oui ; j'ai eu un peu de mal à comprendre... mais bon j'ai fini par comprendre » (5). Enfin une patiente n'a pas adhéré à la forme de ce schéma : «je pense que c'est vraiment une question de personnalité, ça ne me parle pas. Il y a des gens pour qui le graphique va leur parler, moi je sais que même amélioré, je pense que je ne l'utiliserai pas »(10).

Les patients interrogés ont également relevé les témoignages comme outil pédagogique. Ils ont estimé qu'ils facilitaient l'appropriation du contenu : «c'est plus parlant d'avoir affaire directement à des diabétiques, on est tout de suite dans le concret donc ça parle tout de suite» (3) et la mémorisation : «ils m'ont aidé à vraiment retenir les informations » (4).

Ils ont été souvent appréciés par les patients : «j'ai bien aimé les témoignages des gens, c'était sympa» (2); "ce que j'ai apprécié le plus ce sont les témoignages, oui ça franchement j'ai apprécié» (3); «ce que j'ai beaucoup apprécié aussi c'est les témoignages des personnes » (5).

D'abord parce qu'il s'agissait de la parole d'autres personnes diabétiques de type 1 : «c'est agréable d'avoir l'avis de personnes diabétiques » (2) ; «on regarde pour avoir un autre point de vue »(8).

Cette confrontation leur a permis de sortir d'un certain « isolement » face à la maladie : "ça m'a permis de voir que je n'étais pas tout seul dans ce cas-là » (3) ; "je n'ai jamais rencontré d'autres diabétiques de type 1, jamais pu parler de ce qu'elles vivaient, comment elles le ressentaient $»(11)$.

Certains ont apprécié la diversité des témoignages leur permettant de retrouver des situations vécues : «ce que eux racontent par rapport à ce qu'ils peuvent ressentir ou peuvent vivre, j'ai trouvé que c'était juste » (3) ; «je me sentais beaucoup plus concernée en fait quand c'étaient les jeunes. Par exemple celui qui se dit «Est-ce que je vais sortir de l'amphi ou pas? », celui qui se dit «Le carnet je n'ai pas envie de le remplir ». C'est des trucs qui me passent par la tête » (5); "il y a différents cas de figure au niveau des témoignages, on peut s'y retrouver car il y a différentes tranches d'âges, différentes personnes ; c'était très intéressant parce que ce sont des témoignages de diabétiques donc c'est vrai qu'on peut se retrouver dans certaines situations. Au fil des témoins il y a un événement ou une réflexion qu'on s'est faite» (7); "je ne me suis pas identifié à un 
témoin en particulier mais il y a des situations que l'on vit dans les différents témoignages » (8); "on peut s'identifier, parce que c'est à peu près les mêmes soucis, les mêmes préoccupations »(11).

Une patiente a souligné la nécessité de varier les témoignages : «heureusement qu'il y a plusieurs témoignages parce que chaque situation est tellement différente que ça peut faire culpabiliser si on ne se retrouve pas dans les témoignages »(7).

Bien qu'il ait apprécié les témoignages, le patient $n^{\circ} 3$ a nuancé ses propos. Il a répondu «non » à la question $\mathrm{n}^{\circ} 19$ : « Les exemples utilisés sont-ils proches des situations que vous vivez au quotidien ? »Il a précisé : «je ne dirais pas que c'était proche de ma situation actuelle. Déjà il y avait pas mal de gens qui pratiquent une activité sportive ce qui n'est pas mon cas. Les gens parlaient de leurs hypoglycémies, moi je n'en fais pas »(3).

D'autres patients ont apporté quelques nuances à leur discours. Une patiente a évoqué le fait que les témoignages pouvaient parfois être troublants : «il y a un témoin qui disait qu'elle ne voulait jamais être au-dessus de 1. Si je l'avais entendue il y a quelques mois ça m'aurait fait peur. Moi je les connais mes objectifs glycémiques, je n'avais pas forcément envie d'entendre peut-être des témoignages de gens qui font mieux que moi » (7).

Un patient quant à lui a déclaré avoir parfois trouvé les témoignages un peu longs : «les vidéos parfois c'était un tout petit peu long, une fois que l'on s'aperçoit que c'est le même ressenti, le même cadre, on s'attarde plus sur les schémas précis pour approfondir. Les témoignages on les prend plus comme soutien psychologique, les textes c'est plus précis » (8).

Une autre patiente a avoué avoir eu des difficultés à s'identifier : "moi en vidéo ça me parle moins parce que même si c'est la même situation je ne me reconnais pas vraiment dans la personne »(9).

A la question $n^{\circ} 16:$ " Vous êtes-vous senti déstabilisé dans la gestion de votre diabète au quotidien ? », trois des patients interrogés ont répondu être plutôt d'accord.

Le patient $\mathrm{n}^{\circ} 2$ a expliqué : «je ne sais pas trop pourquoi j'ai coché ça, mais c'est un peu entre les deux. Peut-être avec les seuils de tolérance qu'ils fixent en fait dans les glycémies. Quand par exemple après le repas j'ai une glycémie à 0,8 ou 0,9. Sur le site ils conseillent vraiment à partir de 1,2... enfin je sais plus les valeurs, mais du coup ça m'avait un peu déstabilisé. »

La patiente $\mathrm{n}^{\circ} 6$ a déclaré : «il y a toujours un petit peu des doutes. Par exemple la personne, le bûcheron, c'est une personne qui descendait très bas dans sa glycémie et ça 
ça m'a beaucoup interpellée parce que je me suis demandé si ce qu'il faisait c'était bien ou c'était pas bien... Bon après j'ai eu des réponses. »

La patiente $\mathrm{n}^{\circ} 9$ a dit : «moi ce qui me fait vraiment peur dans le diabète ce sont les complications. Du coup c'est les hypers qui me font vraiment peur. Et le $1^{\text {er }}$ chapitre m'a... enfin ça m'a un peu permis de relativiser par rapport à ça. Que bon voilà, il y a des moments où on parle de résultats de plus de 2 grammes et ça a l'air presque humain. J'ai été déstabilisée mais de façon positive, ça m'a permis de prendre du recul, de voir ça autrement au quotidien, de relativiser certaines choses et d'être plus attentive sur d'autres. »

Les patients interrogés ont déclaré que le site INDIGO pouvait susciter des interactions avec leur entourage.

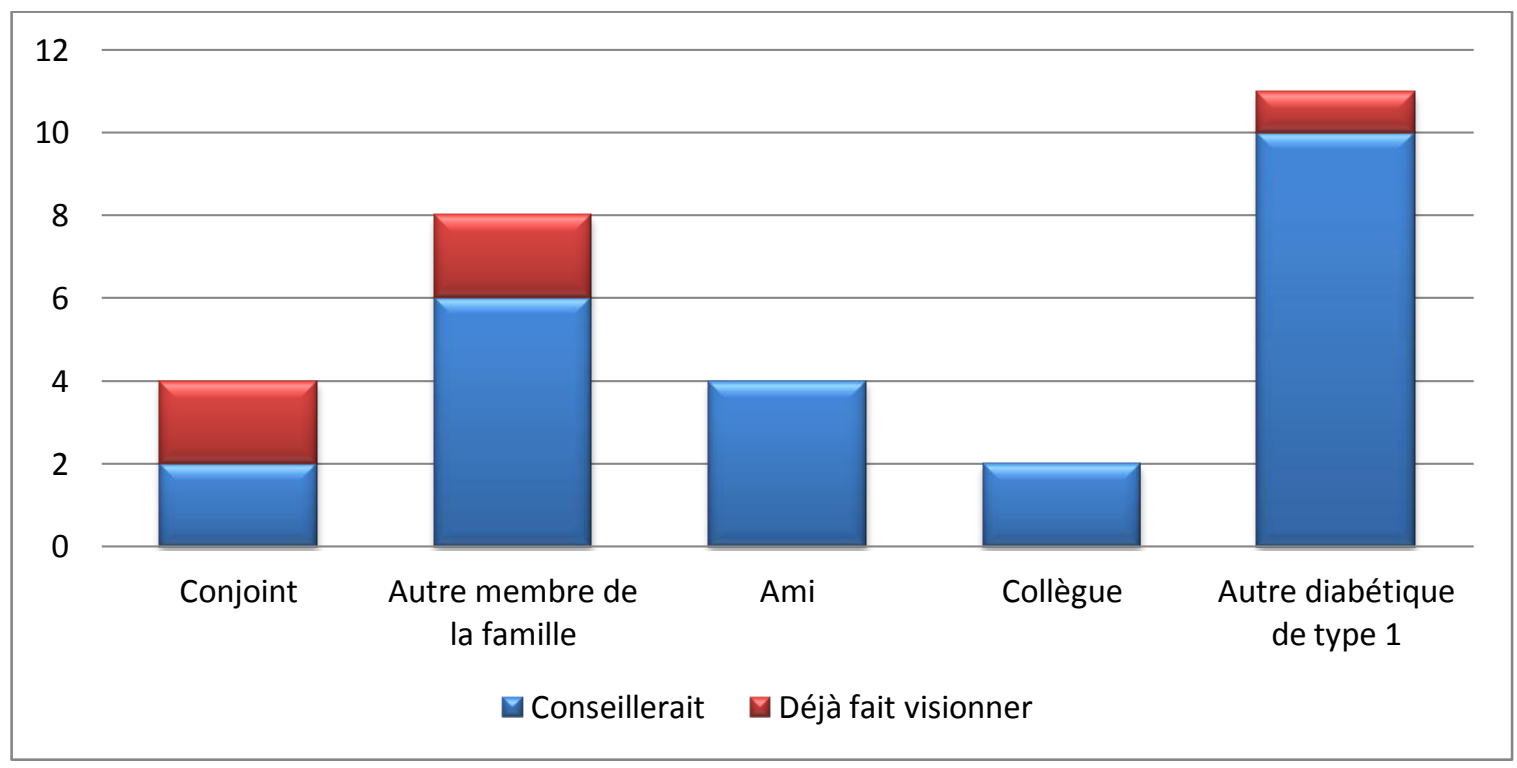

Figure 23 : A quels membres de leur entourage les patients ont-ils fait visionner ou conseilleraient-ils le site INDIGO?

2 patients ont déclaré qu'ils conseilleraient le site INDIGO à leur conjoint, 6 à un autre membre de leur famille, 4 à des amis, 2 à des collègues et 10 à un autre patient diabétique de type 1.

Ils ont expliqué que le site INDIGO pourrait être utile pour leur faire comprendre ce qu'est le diabète : "pour les compagnes, les gens qui accompagnent, c'est quand même pas mal, ça permet de mieux comprendre la maladie» (2) ; "pour qu'il comprenne réellement ce qu'est mon diabète parce qu'il y a des choses qu'il ne saisit pas encore totalement » (6); «c'est tellement pédagogique je trouve que ça permet aux personnes de 
mieux comprendre les hypos, les hypers, l'insuline ; ça peut être un outil pour la personne proche qui se pose des questions de fond sur le diabète » (7).

Ils ont pensé que le site INDIGO pourrait également les rassurer : «je le montrerai à ma mère par exemple qui panique un peu, enfin, qui n'est pas à l'aise avec le sujet, ça peut la rassurer » (1); "pour rassurer ma famille, confirmer que l'on peut vivre avec le diabète sans trop de soucis, faire du sport, manger ce que l'on veut ; qu'il n'y a pas de limitation à la vie de tous les jours » (4) ; "ma mère qui est très stressée avec le diabète je pense que ça la déstresserait; les témoignages notamment où on se rend compte que les gens vont bien, s'en sortent, vivent bien »(7).

Un patient a noté le fait que le site INDIGO pourrait présenter à l'entourage un discours superposable au sien : «il pourrait voir que c'est pareil pour les autres; il pourrait se rendre compte qu'il n'y a pas de modification de discours, qu'on n'aggrave pas, qu'on ne relativise pas » (8).

Une patiente a souligné la possibilité d'informer son conjoint sans devoir le faire ellemême : "j'aurais aimé que mon copain le regarde avec moi mais c'est vrai que je ne lui parle jamais de... Ça reste quelque chose que je garde pour moi. Mais c'est vrai qu'il n'a aucune notion. Je pense que le site j'essayerai de lui montrer quand même parce que c'est des choses que je n'ai pas envie de lui expliquer. En fait je n'ai pas envie d'en parler vraiment. Mais il y a certaines choses qu'il faut qu'il sache»(9).

Enfin une patiente a estimé que le site INDIGO pourrait être utile pour l'entourage d'une personne diabétique mais n'a pas envisagé de le montrer au sien : «pour l'entourage d'une personne diabétique je pense que c'est très bien. Ça serait bien pour leur faire comprendre. Mais moi personnellement je ne voudrais pas; je ne le regarderais pas avec quelqu'un d'autre, je ne sais pas si c'est parce que je n'accepte pas que je suis diabétique. Et puis je ne supporte pas que l'on s'occupe de moi donc je pense que si je le montrais ça les alarmerait plus qu'autre chose. Le risque de coma c'est pas des trucs que je leur ai dit, les dangers, les conséquences si on ne fait pas comme il faut... »(11).

Deux patients avaient déjà fait visionner le site INDIGO à leur conjoint.

Le patient $\mathrm{n}^{\circ} 2$ a relaté : «c'était agréable pour elle vu qu'elle est moins au courant que moi de ma maladie, elle a appris beaucoup. »

La patiente $\mathrm{n}^{\circ} 7$ a rapporté : «Mon compagnon a trouvé ça intéressant, après par rapport à moi il ne va pas regarder tous les chapitres mais ça permet de répondre à des questions. » 
La patiente $n^{\circ} 10$ l'avait montré à sa mère : «elle l'a tout de suite regardé sans que je lui demande quoi que ce soit; pour tout ce qui est Glucagon c'était super parce que ça a vachement rassuré ma mère, le fait de le voir c'est beaucoup plus rassurant qu'à l'oral. Elle a trouvé que c'était parlant, elle a bien aimé le site, elle a gardé les codes » et à sa sœur: "la séquence du Glucagon elle m'a dit que le fait de le voir c'est un peu dédramatisant parce qu'on se dit que ce n'est pas bien compliqué. »

Un patient l'avaient fait visionner à ses parents qui vivent en Italie : «j'ai essayé, ils m'ont demandé de traduire une partie, mais après bon, il y a quatre heures de chapitres... Ils ne sont pas chez moi donc... »(4).

Enfin une patiente avait montré le site INDIGO à son frère, lui-même diabétique de type 1 : «il a bien aimé parce que comme moi il y a des choses qu'il ne comprend pas tout à fait donc ça permet de faire un point. Il me disait : c'est clair, c'est coloré, on peut participer, c'est animé et c'est un bon choix de support » (5).

Les patients ont donc rapporté que les membres de leur entourage qui avaient visionné le site INDIGO l'avaient apprécié et qu'il semblait en effet être un bon support pour leur expliquer le diabète et pour les rassurer.

Les patients interrogés ont déclaré que le site INDIGO pouvait également susciter des interactions avec leur diabétologue.

Certains patients ont évoqué le besoin de revoir certaines notions avec lui : «peut-être revoir avec lui les intervalles de glycémie qu'il faut avoir car je fais pas mal de sport aussi. Ça sera à discuter avec lui, ça sera une bonne occasion » (2) ; " j'ai tout imprimé comme ça au moins j'étais sûre, je lui ai fait part de cette petite observation et il m'a bien expliqué que c'était bon » (3) ; "dans le chapitre 1 il y a des valeurs explicites qu'il faut par contre vérifier et un peu affiner au cas par cas avec son diabéto » (4); "j'ai eu besoin de lui demander si par rapport à comment je l'avais perçu sur le site j'allais faire le bon geste, du coup elle m'a bien confirmé, elle m'a bien rassurée » (6) ; "je pense qu'il faut en discuter parce que du coup il faut redéfinir vraiment les objectifs » (9) ; «mes objectifs glycémiques c'était quelque chose que je m'étais dit que je reverrai justement avec ma diabéto »(10).

Certains patients ont évoqué le fait que le site INDIGO pouvait les aider à définir des questions, à préparer une consultation : «il m'a aussi stimulé pour trouver des autres questions que je vais poser à un diabétologue la prochaine fois que je le vois; les 
questions qui étaient là quelque part dans ma tête ça a aidé un peu à les formaliser » (4) ; «donne des infos qui peut-être justement dégrossiraient l'information avant de poser des questions à notre diabéto en consultation »(8).

Un patient a souligné la possibilité d'utiliser le site INDIGO pour répondre à certaines questions, au moins de manière partielle, afin de pouvoir attendre la prochaine consultation : "ça peut être bien de le voir si l'on a une question sur le diabète, ça peut suffire et faire patienter jusqu'à la visite suivante »(8).

Une patiente a évoqué le manque de la relation médecin-patient mais a nuancé ses propos : «il manque toujours la relation entre le diabétologue et le patient mais avec le recul je me dis que c'est bien que moi je vive mon diabète, que je me pose 10000 questions qui me permettent de prendre des décisions. Parce que moi mon trait de caractère c'est que des fois je ne me fais pas confiance, j'ai des doutes sur ce que je pense. Mais c'est bien de pouvoir en parler à un moment donné »(6). 


\subsection{Appréciations relatives à la qualité du support}

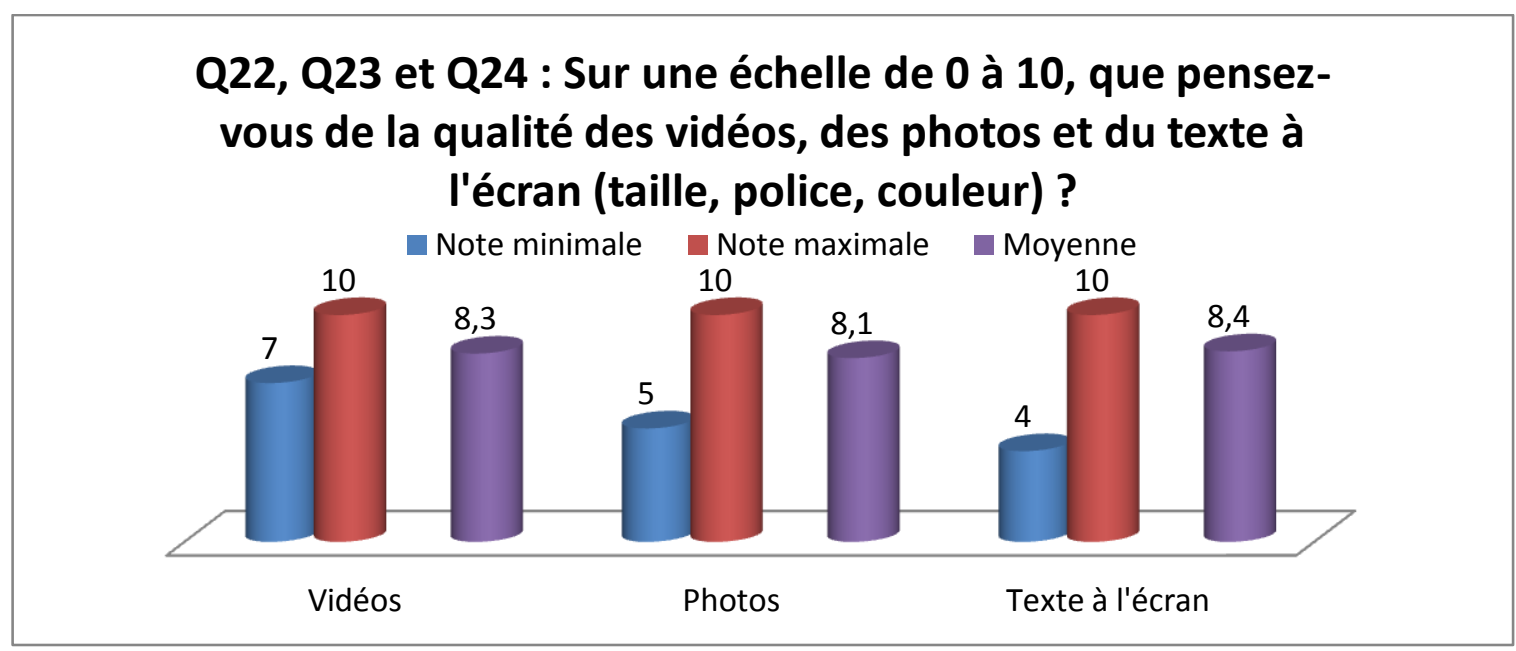

Figure 24 : Réponses aux questions $n^{\circ} 22,23$ et 24

Les patients interrogés ont attribué la note moyenne de 8,3/10 à la qualité des vidéos. Les notes variaient de 7 à 10/10 (cf. Annexe 7).

Ils ont attribué la note moyenne de 8,1/10 à la qualité des photos. Les notes variaient de 5 à 10/10. La note de 5/10 a été attribuée par la patiente $\mathrm{n}^{\circ} 11$. Les autres notes étaient supérieures à 7/10 (cf. Annexe 7).

Ils ont attribué la note moyenne de $\mathbf{8 , 4 / 1 0}$ à la qualité du texte à l'écran. Les notes variaient de 4 à 10/10. La note de $4 / 10$ a été attribuée par le patient $n^{\circ} 2$, la patiente $\mathrm{n}^{\circ} 7$ a attribué la note de 5/10. Les autres notes étaient supérieures à 7/10 (cf. Annexe 7).

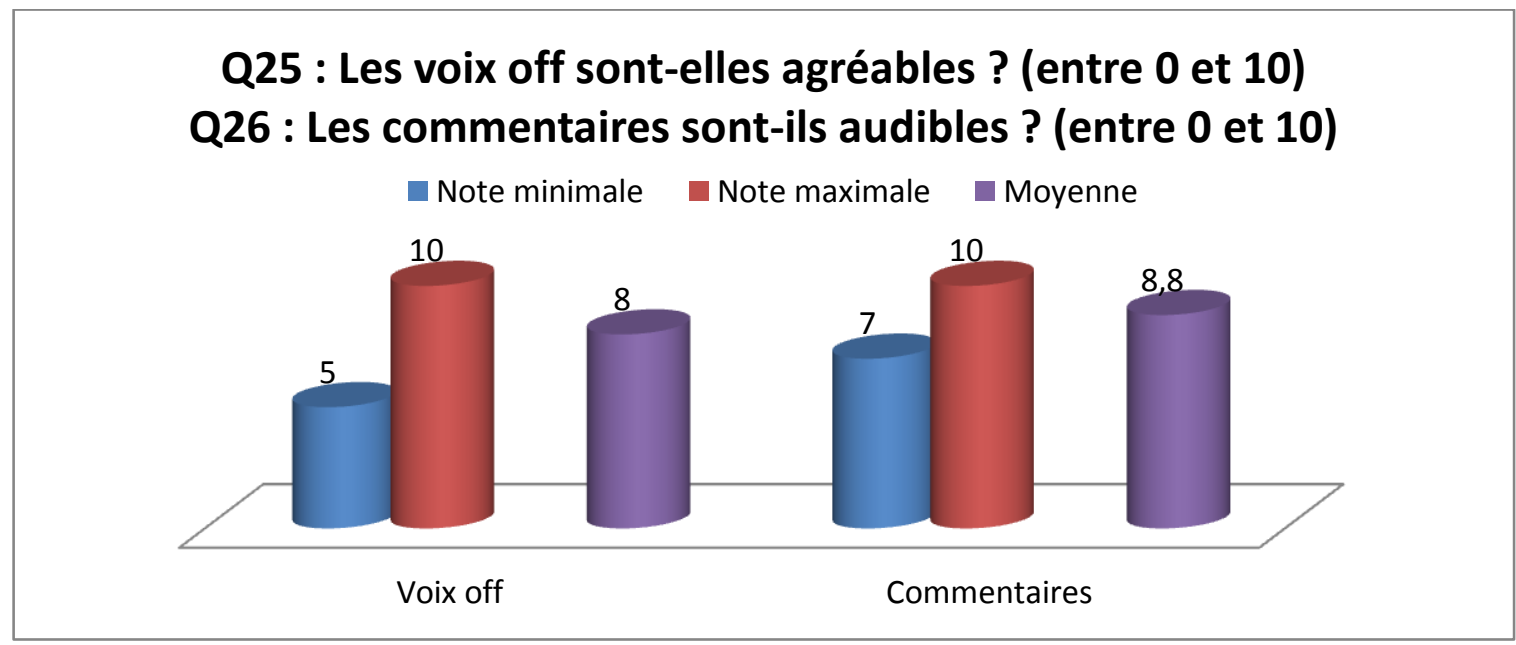

Figure 25 : Réponses aux questions $n^{\circ} 25$ et 26

A la question «Les voix off sont-elles agréables ? » la note moyenne attribuée par les patients interrogés a été de 8/10. Les notes variaient de 5 à 10/10. Les patients ${ }^{\circ} 10$ et 11 ont attribué la note de 5/10. Les autres notes étaient supérieures à 7/10 (cf. Annexe 7). 
A la question « Les commentaires sont-ils audibles ? » la note moyenne attribuée par les patients interrogés a été de 8,8/10. Les notes variaient de 7 à 10/10 (cf. Annexe 7).

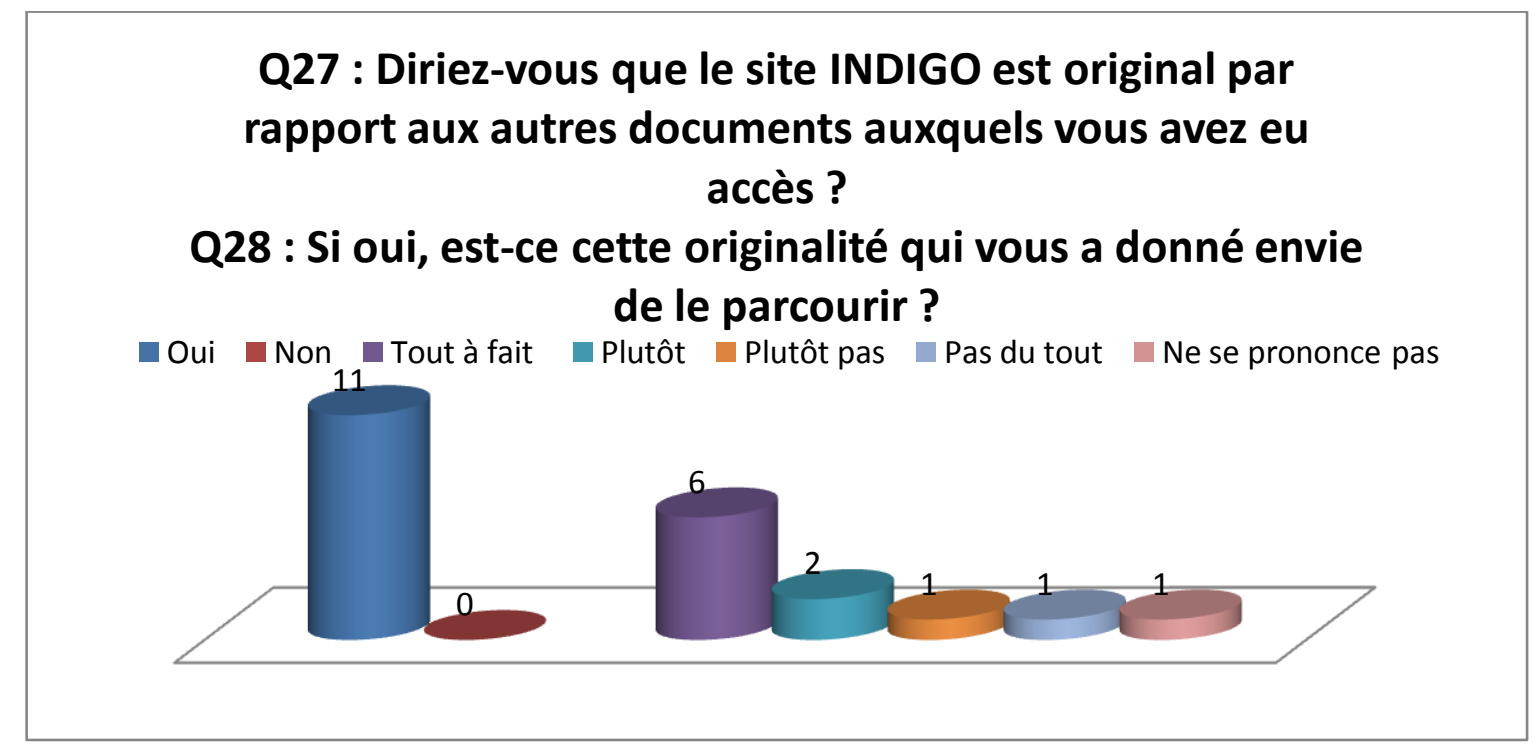

Figure 26 : Réponses aux questions $n^{\circ} 27$ et 28

L'ensemble des patients interrogés a déclaré que le site INDIGO était original par rapport aux autres documents d'information sur le diabète auxquels ils avaient eu accès.

Pour 8 d'entre eux $(72,7 \%)$ c'était cette originalité qui leur avait donné envie de le parcourir.

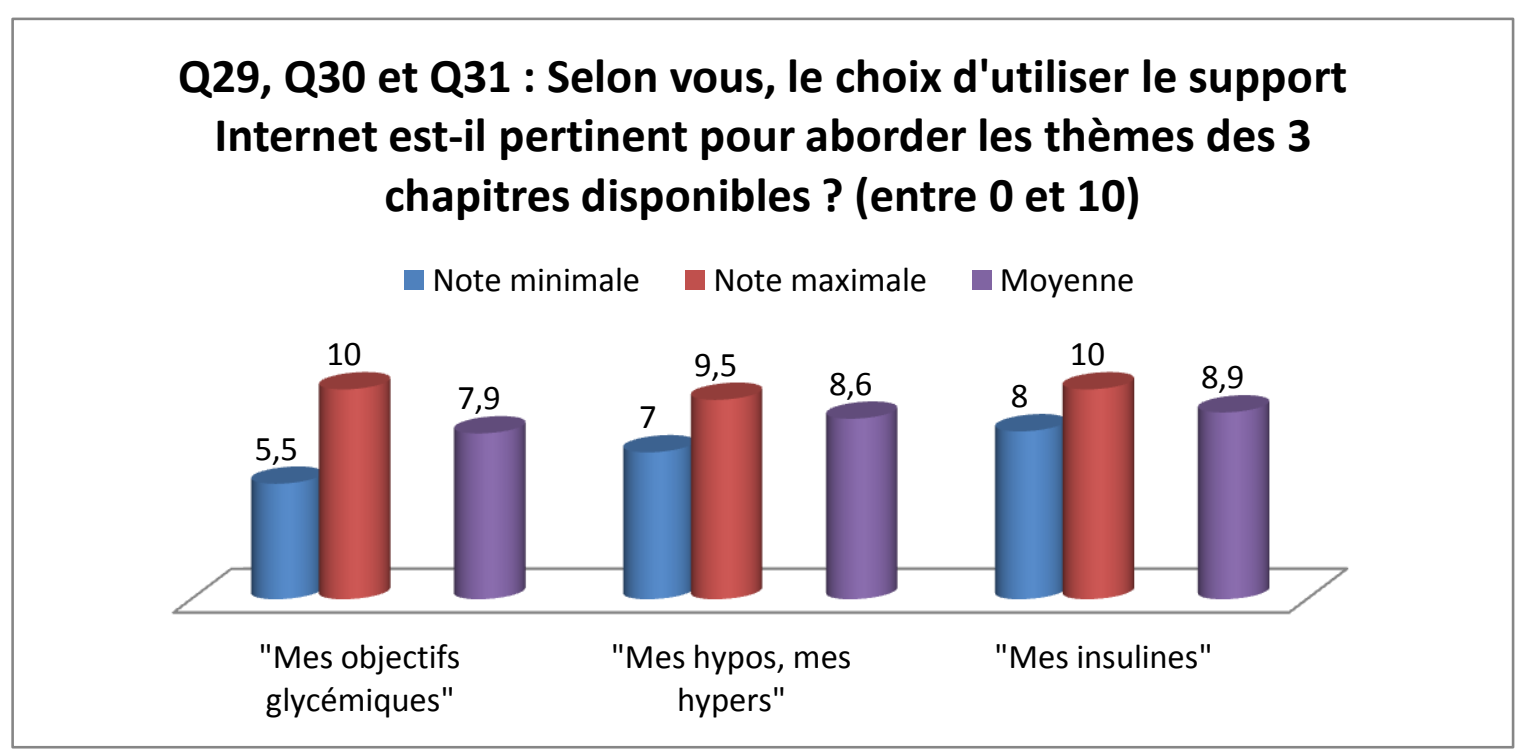

Figure 27 : Réponses aux questions $n^{\circ} 29,30$ et 31

Les patients interrogés ont attribué la note moyenne de 7,9/10 à la pertinence du choix d'utiliser le support Internet pour aborder le thème du chapitre «Mes objectifs glycémiques ». Les notes variaient de 5,5 à 10/10. La note de 5,5 a été attribuée par les patients 5 et 7 . Les autres notes étaient supérieures à 7 (cf. Annexe 7). 
Les patients interrogés ont attribué la note moyenne de 8,6/10 à la pertinence du choix d'utiliser le support Internet pour aborder le thème du chapitre «Mes hypos, mes hypers ». Les notes variaient de 7 à 9,5/10 (cf. Annexe 7).

Les patients interrogés ont attribué la note moyenne de 8,9/10 à la pertinence du choix d'utiliser le support Internet pour aborder le thème du chapitre «Mes insulines ». Les notes variaient de 8 à 10/10 (cf. Annexe 7).

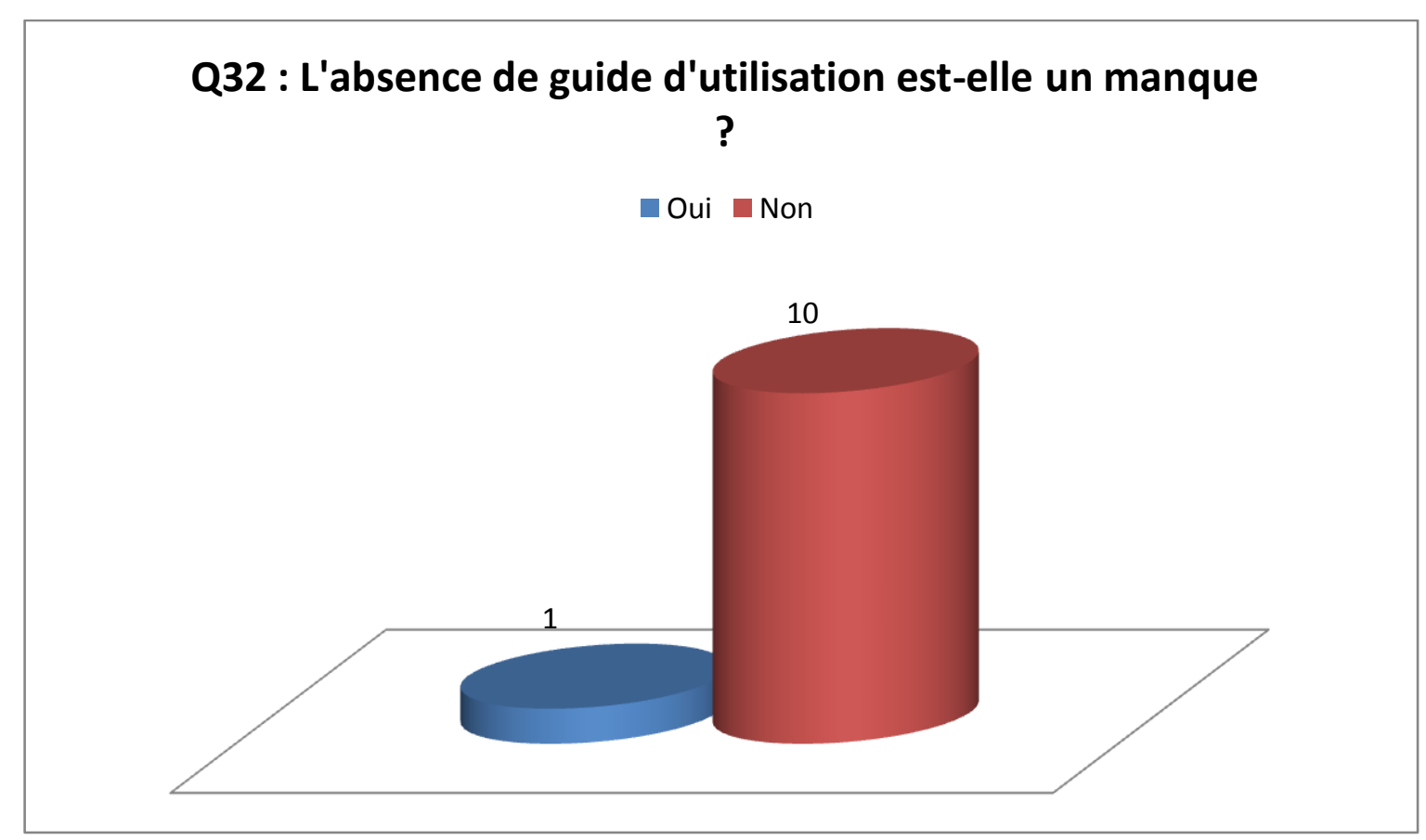

Figure 28 : Réponses à la question $\mathbf{n}^{\circ} 32$

10 patients $(90,9 \%)$ ont estimé que l'absence de guide d'utilisation n'était pas un manque. 


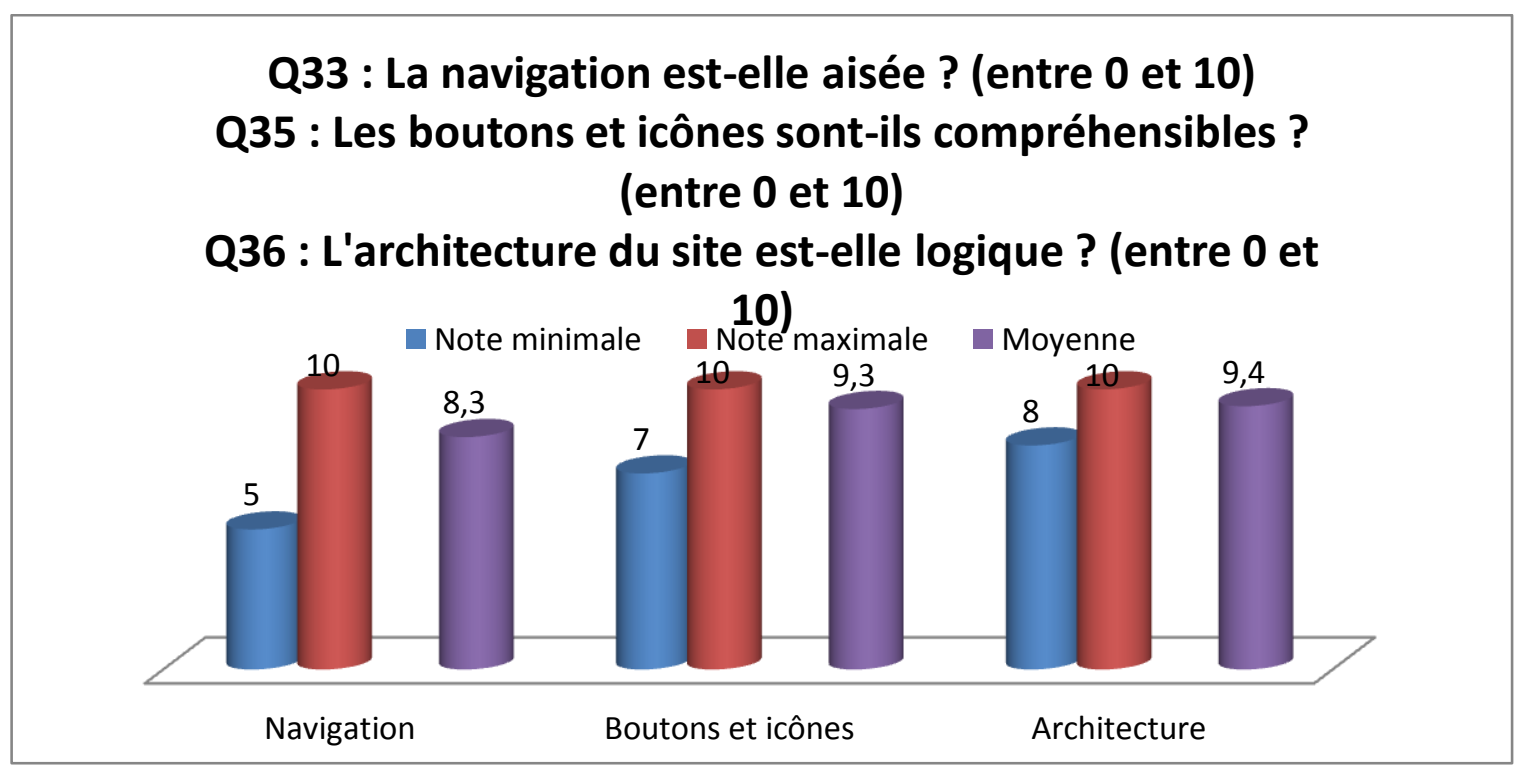

Figure 29 : Réponses aux questions $n^{\circ} 33,35$ et 36

A la question « La navigation est-elle aisée ? » la note moyenne attribuée par les patients interrogés a été de 8,3/10. Les notes variaient de 5 à 10/10. Le patient $\mathrm{n}^{\circ} 2$ a attribué la note de 5/10. Les autres notes étaient supérieures à 6/10 (cf. Annexe 7).

A la question «Les boutons et icônes sont-ils compréhensibles ? " la note moyenne attribuée par les patients interrogés a été de 8,3/10. Les notes variaient de 7 à 10/10 (cf. Annexe 7).

A la question «L'architecture du site est-elle logique? » la note moyenne attribuée par les patients interrogés a été de 9,4/10. Les notes variaient de 8 à 10/10 (cf. Annexe 7).

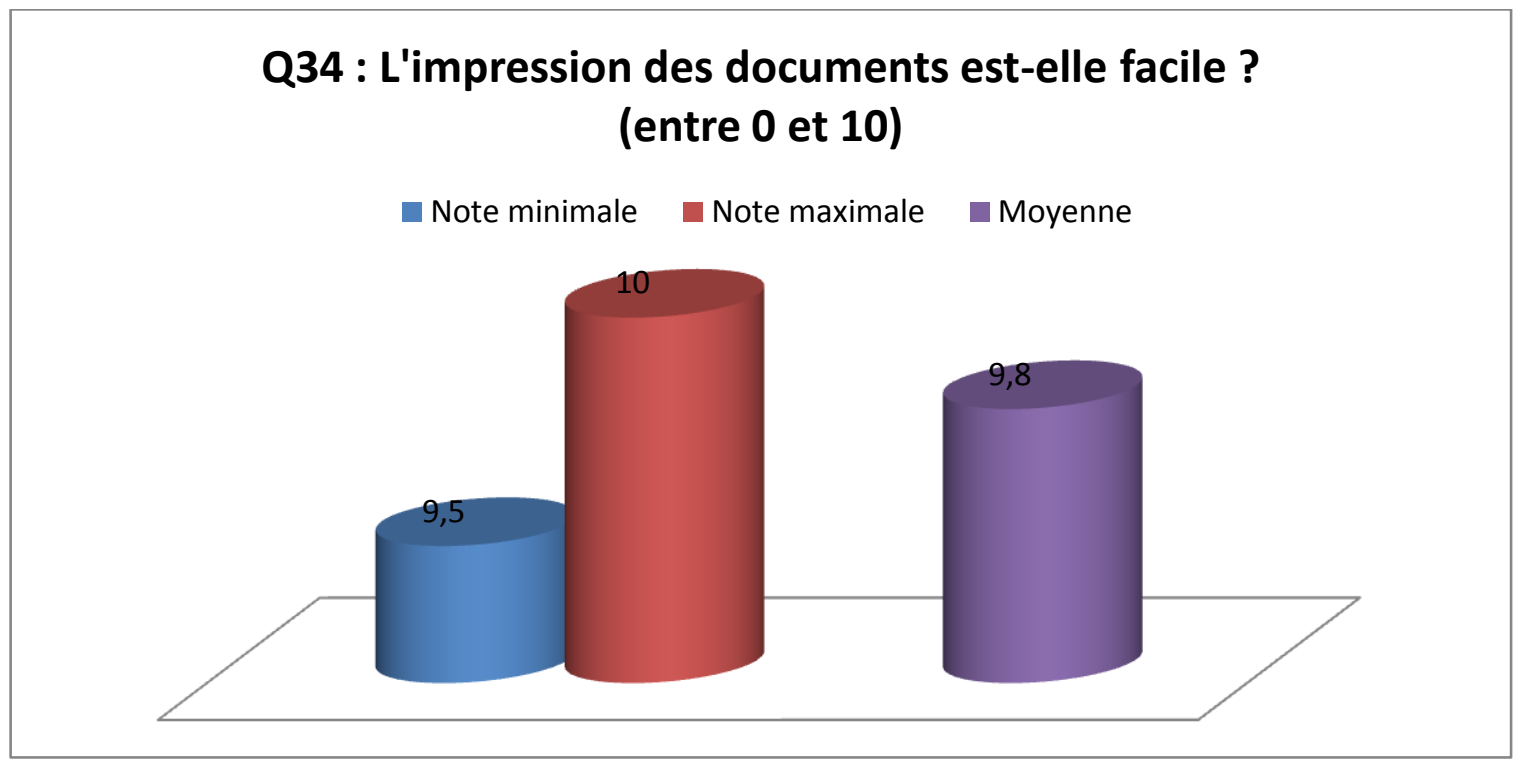

Figure 30 : Réponses à la question n ${ }^{\circ} 34$ 
A la question « L'impression des documents est-elle facile ? » la note moyenne attribuée par les patients interrogés a été de $\mathbf{9 , 8 / 1 0}$. Les notes variaient de 9,5 à 10/10. Il est à noter que 6 des 11 patients interrogés n'ont pas répondu à cette question puisqu'ils n'avaient pas imprimé de document (cf. Annexe 7).

$\mathrm{Au}$ total, les patients interrogés ont estimé que la qualité du support du site INDIGO était en adéquation avec les critères de qualité d'un outil d'intervention en éducation pour la santé de l'INPES. ${ }^{28}$ Ils ont tous attribué une note supérieure ou égale à la moyenne concernant la qualité des vidéos, des photos et des voix off. 90,9\% des patients ont attribué une note supérieure ou égale à la moyenne à la qualité du texte à l'écran. Les commentaires ont été jugés audibles. Le site INDIGO a été perçu comme original par rapport aux autres documents d'information sur le diabète auxquels ils avaient déjà eu accès. Pour la plupart d'entre eux c'était cette originalité qui leur avait donné envie de le parcourir. Le choix d'utiliser le support Internet a été jugé pertinent pour aborder les thèmes des trois chapitres disponibles. L'absence de guide d'utilisation n'était pas un manque pour 90,9\% des patients. Les boutons et icônes ont été estimés compréhensibles, l'architecture du site logique, l'impression des documents facile et la navigation aisée.

L'analyse des entretiens semi-dirigés nous a donné davantage de précisions sur les appréciations des patients interrogés concernant la qualité du support du site INDIGO.

Concernant la qualité des vidéos un patient a indiqué : «les images à l'écran c'était clair, ça prenait une bonne place à l'écran par rapport à ce qu'on voyait. Et puis comme c'était monté, enfin c'était agréable à écouter » (2).

Deux patients ont mentionné une certaine lenteur : «parfois c'est un peu lent dans la vidéo, on a envie de passer vite » (6) ; "les vidéos sont peut-être un peu longues mais le fait qu'on puisse zapper à ce moment-là il n'y a pas de soucis » (8).

Une des patientes a évoqué une absence de modernité dans la façon de filmer : «je trouve que ça manque un peu de vie au niveau des témoignages, c'est assez statique. Il manque une façon de filmer plus moderne et attractive. Elle a nuancé ses propos : en même temps c'est un outil thérapeutique pas un spot publicitaire » (7).

Plusieurs patients ont regretté la qualité du jeu : «ce qui m'a fait un peu rire c'était la scène quand la personne s'endort devant la télé. Après c'est normal ce ne sont pas des 
acteurs mais c'est un peu sur joué... donc c'est ça qui est marrant» (2) ; «il y a un témoignage que j'ai trouvé un peu sur joué, c'était le cas du jeune qui balance son carnet et qui crie "Oui, j'en ai marre de ce diabète ». Ça m'a fait rire » (3) ; "ça ne fait pas vraiment vrai, ça fait acteur. C'est peut-être parce que je connaissais les gens dans la vidéo. Le jeune homme était dans mon lycée et Manon, elle fait la femme enceinte, en fait c'est une amie à moi. Mais les vidéos sont claires, je ne pense qu'il faille le changer » (9) ; "les vidéos ça me faisait rigoler parce que moi je trouvais que c'était très mal joué. Je trouve que ça ne fait pas très sérieux mais bon ça explique bien, d'un côté c'est ce qu'il faut $\gg(11)$.

Un patient a apprécié la qualité du texte à l'écran : «les couleurs c'était assez attrayant» (3). Le patient $\mathrm{n}^{\circ} 2$ qui a attribué la note de $4 / 10$ a précisé : «les couleurs sont peut-être un peu flashy, le jaune des flèches « suivant » en contraste avec le bleu clair derrière ça fait un peu trop flashy. Le jaune comme ça, très bien, mais peut-être juste la couleur, diminuer l'accentuation» (2). La patiente $\mathrm{n}^{\circ 7}$ qui a attribué un 5/10 a fait des commentaires positifs : «c'est clair, coloré, le texte est de bonne taille, vraiment très lisible. Au niveau des couleurs c'est bien » (7).

A la question $\mathrm{n}^{\circ} 25:$ «es voix off sont-elles agréables ? » deux patientes ont attribué la note de 5/10. L'une n'a pas fait de commentaire. L'autre a déclaré : «un truc que j'ai moins aimé c'était la voix de la personne qui parlait, j'ai demandé à mon fils, il a 12 ans, il m'a dit: elle est pourrie la voix. Elle n'est pas désagréable non plus, je ne sais pas comment l'expliquer »(10).

Une patiente aurait souhaité que le site soit entièrement sous-titré : «toutes les informations ne sont pas écrites. Sur certains appareils je n'ai pas forcément le son qui peut sortir. Je penserais peut-être à ça qui peut poser problème. J'ai un ordinateur chez moi qui est un vieil ordinateur qui n'a pas d'enceintes par exemple. Si je l'avais regardé dessus je serais passée à côté de beaucoup de choses » (1).

Six patients ont apprécié le choix du support Internet.

D'abord pour sa facilité et sa rapidité d'accès : «c'est facile d'accès » (1); "on peut accéder à cette information de manière facile avec Internet car maintenant tout le monde 
est prêt, peut y arriver » (4); " c'est vite accessible, facilement, de chez nous. Surtout que c'est des moments où on a envie tout de suite d'avoir des réponses » (7).

Ensuite parce que cela permet de revenir sur le site INDIGO : «j'apprécie de l'avoir sous la main, de pouvoir y retourner n'importe quand et de pouvoir retrouver les informations chez moi » (6) ; "je vais l'avoir en favori sur mon ordi et je pense que je vais le regarder régulièrement, vraiment au moment de questions particulières » $(7)$; « pour retourner voir des choses, je pense que plutôt que d'avoir un papier ou un carnet que l'on va peut-être perdre, c'est peut-être bien de l'avoir sous forme de site justement. Et puis le nom on s'en souvient bien, les codes d'accès aussi » (8).

Une patiente a souligné le fait que cela lui conférait un caractère original et attrayant : «c'est sympa par rapport à tout ce qui est support papier qui fait tout de suite plus formel, je trouve que ça donne plus envie ; j'ai tendance à ne pas vouloir y penser, me pencher dessus alors que c'est important. Ça m'a permis de me plonger dedans sans avoir l'impression de le faire » (9).

Quatre patients ont également souligné l'interactivité du site INDIGO : « il y a différentes activités, c'est interactif, on s'implique un peu dans le processus. Ce n'est pas juste un dossier qu'on lirait, il y a moyen d'interagir avec » (5); "on peut rentrer ses objectifs glycémiques, c'est interactif » (7) ; "il est très bien pensé, c'est bien fait, c'est interactif. Ça fait un peu site pour enfants, de jeux ou... c'est ludique. J'ai aussi aimé le fait qu'il y ait autant de participation et qu'à la fin on puisse avoir un récapitulatif de ses objectifs personnels de glycémie » (9) ; «le fait de poser des questions comme ça je trouve que c'est interactif $\gg(10)$.

Toutefois, trois patients ont rapporté avoir eu certaines difficultés. Avec les codes d'accès d'abord : "j'ai voulu le regarder mais on demandait le code et je ne m'en souvenais pas » (9); avec le navigateur ensuite : «il n'est pas accessible sur Google, c'est sur Chrome, donc il a fallu que je le télécharge, ça s'est bien fait mais du coup ça m'a surprise » (1); "je n'avais pas utilisé le bon navigateur. Ça ne m'a pas gênée plus que ça que ce ne soit pas disponible sur tous les navigateurs, mais si vous ne m'aviez pas appelée je ne sais pas si je serais allée plus loin »(10).

A la question $\mathrm{n}^{\circ} 32$ : «L'absence de guide d'utilisation est-elle un manque ? », un patient a répondu « oui ». Il a précisé : «il faudrait peut-être au lancement du site, au menu, dire que le site INDIGO fonctionne avec de l'audio. C'est peut-être un petit truc à mettre sur le côté parce que j'ai l'habitude de ne pas mettre de son sur mon ordi et quand je suis arrivé 
sur le site il y avait la voix off mais je ne comprenais pas ce qu'il se passait. Après je me suis rendu compte qu'il fallait activer l'audio » (2).

Deux patients ont apprécié la facilité de navigation : «il est très facile d'utilisation, même pour des personnes pour qui ce n'est pas forcément naturel de naviguer sur un site comme ça. Et au niveau des façons de cliquer c'est très bien fait, très bien pensé » (7) ; "quand on rentre dans un chapitre il n'y a pas grand chose à faire, c'est guidé. Je trouve que ça fait un peu jeu vidéo. Il y a des moments où c'est quand même contrôlé, il y a des moments où il faut participer » (9).

Un patient a aimé le fait de pouvoir consulter les chapitres dans le désordre : «c'est bien que l'on puisse faire dans le désordre, laisser de côté, revenir »(3).

A la question $\mathrm{n}^{\circ} 33$ «La navigation est-elle aisée ? » le patient $\mathrm{n}^{\circ} 2$ a attribué la note de 5/10. Il a regretté le trop grand découpage des sous-chapitres : «ce qui est dérangeant c'est que la voix off dit une phrase et qu'il faut cliquer sur « suivant », « suivant ». Parfois elle parlait 5 à 10 secondes et on proposait de cliquer. Ça casse le rythme. Il faudrait peutêtre intégrer un ou deux passages à celui d'avant ou d'après. Il a nuancé ses propos : mais ne pas tout faire d'un bloc parce que si on veut directement aller à tel endroit c'est pas mal que ce soit étape par étape. » Il a également regretté l'absence d'enchainement spontané entre les chapitres : «quand le chapitre 2 était fini il fallait retourner dans le menu pour cliquer sur le chapitre 3, on ne proposait pas d'y aller directement. J'aurais préféré que la flèche dise : "maintenant voulez-vous passer au chapitre 3 ? » 》 De même : «le chapitre 3 est en deux parties et quand on a fini la première partie on ne propose pas de passer à la deuxième, on repropose de revenir au menu pour cliquer sur la deuxième partie. » Enfin il a signalé des difficultés d'affichage : "si on met en mode plein écran et que l'on fait flèche «suivant» de souvenir ça enlève le mode plein écran. Après j'avais pris l'habitude, je ne mettais plus en mode plein écran. C'était très lisible sans. »

Un autre patient a quant à lui estimé que certaines fonctions étaient inutiles : «la possibilité de mettre en plein écran on ne s'en sert pas trop, on peut laisser comme c'est, ça laisse accès au menu. Il faudrait faire un peu plus sobre, enlever ce qu'on peut enlever : tous les petits onglets sous la vidéo à part avancer et reculer ce n'est pas toujours utile. 
Même le bouton "volume », on n'en a pas tellement besoin parce qu'à la limite le volume on peut le régler de l'ordinateur »(8).

Cinq patients ont imprimé certains documents. Une des patientes a regretté le fait que les documents à imprimer ne soient pas répertoriés dans le menu : "il faut que l'on refasse tout le chapitre pour imprimer les documents. Dommage qu'il n'y ait pas dans le sommaire un onglet pour les documents à imprimer»(10). Elle a regretté également le format d'impression : «ce qui est dommage avec le carnet INDIGO c'est qu'il n'est pas au format A4, il est petit quand on l'imprime. J'ai dî faire un bric-à-brac pour l'agrandir » (10).

Quatre patients ont rencontré des problèmes techniques en visionnant le site INDIGO : " on a des items à remplir et je crois qu'à un moment, mais peut-être que c'est moi ou mon ordi je ne sais pas, mais il y a eu une sorte de bug. Je voulais cocher les cases et ça ne se cochait pas» (3); "ça plante de temps en temps, hier je n'ai pas pu y accéder par exemple. La page se chargeait tout le temps et pourtant j'utilise Safari. La première fois ça avait marché super. La deuxième pas top, hier pas top et aujourd'hui ça va» (8); «quelque fois pour revenir au menu il y avait de l'attente mais ça vient peut-être de ma connexion » (9) ; «au début on n'arrivait pas à le visionner en fait parce que justement on avait les voix qui buggaient, ça a bien duré un quart d'heure et puis après d'un coup elle a pu visionner la totalité ; il y a un temps d'attente à chaque fois, il y a le loading qui se met en route, des fois entre chaque plan c'était un petit peu long, mais c'est peut-être lié à mon ordinateur $\gg(10)$. 


\subsection{Appréciations relatives à la qualité de la conception}

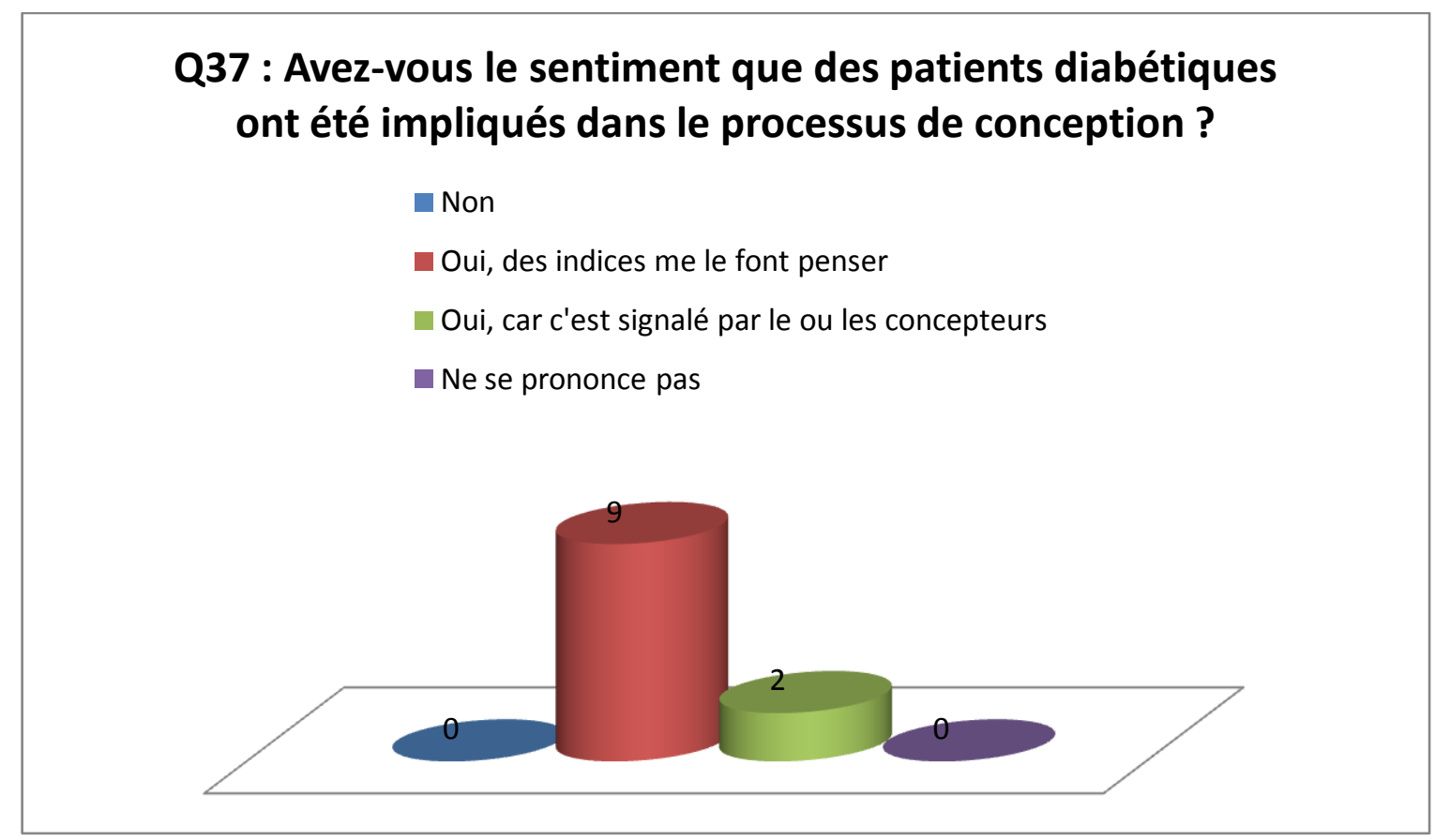

Figure 31 : Réponses à la question $\mathbf{n}^{\circ} 37$

Les patients interrogés ont déclaré avoir eu le sentiment que des patients diabétiques avaient été impliqués dans le processus de conception du site INDIGO.

L'analyse des entretiens semi-dirigés ne nous a pas apporté d'informations supplémentaires sur les appréciations des patients interrogés concernant la qualité de conception du site INDIGO. 


\subsection{Appréciation d'ensemble}

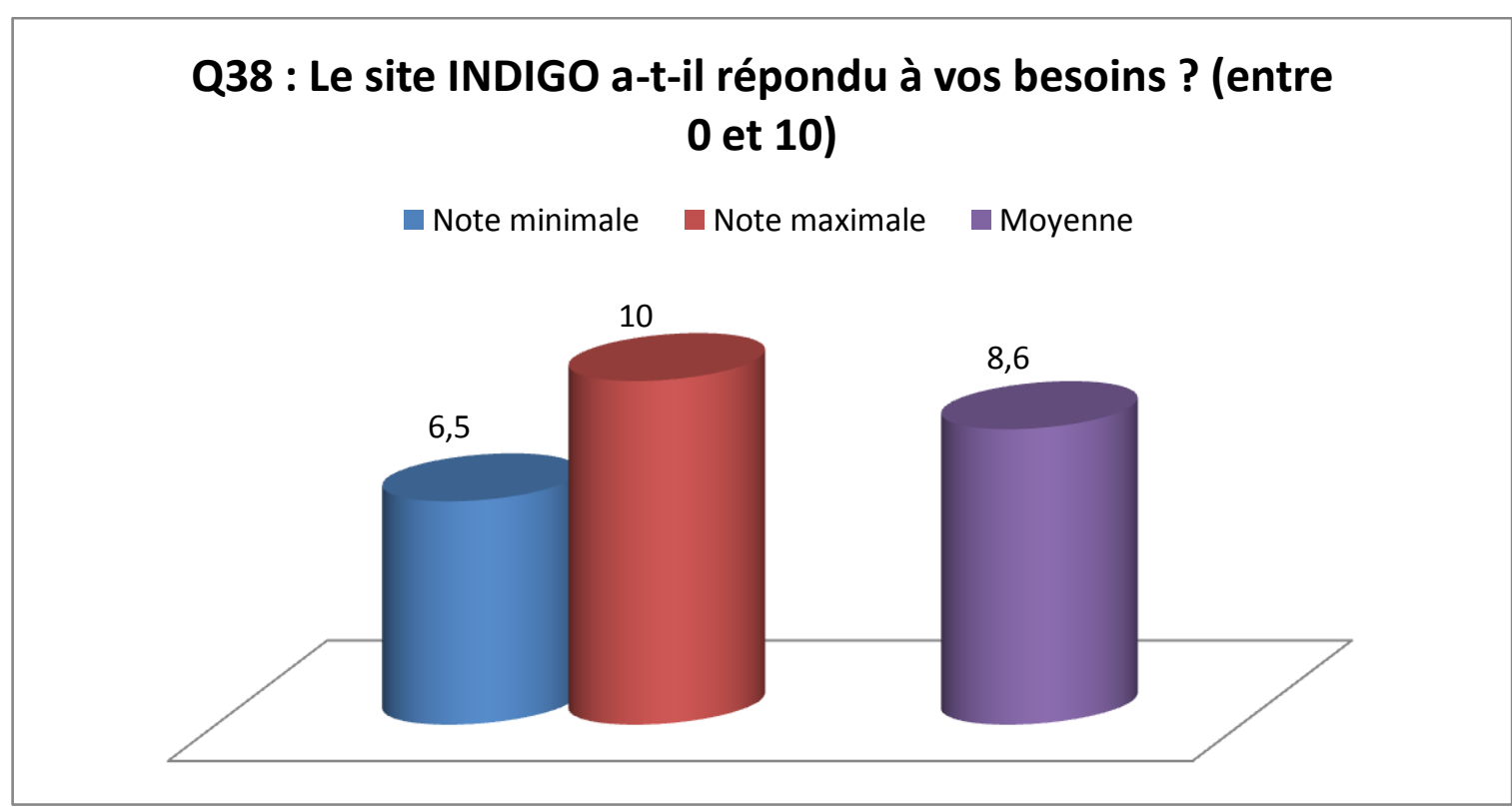

Figure 32 : Réponses à la question $\mathbf{n}^{\circ} 38$

Les patients interrogés ont considéré que le site INDIGO avait répondu à leurs besoins avec une note moyenne de 8,6/10. Les notes variaient de 6,5/10 à 10/10 (cf. Annexe 7).

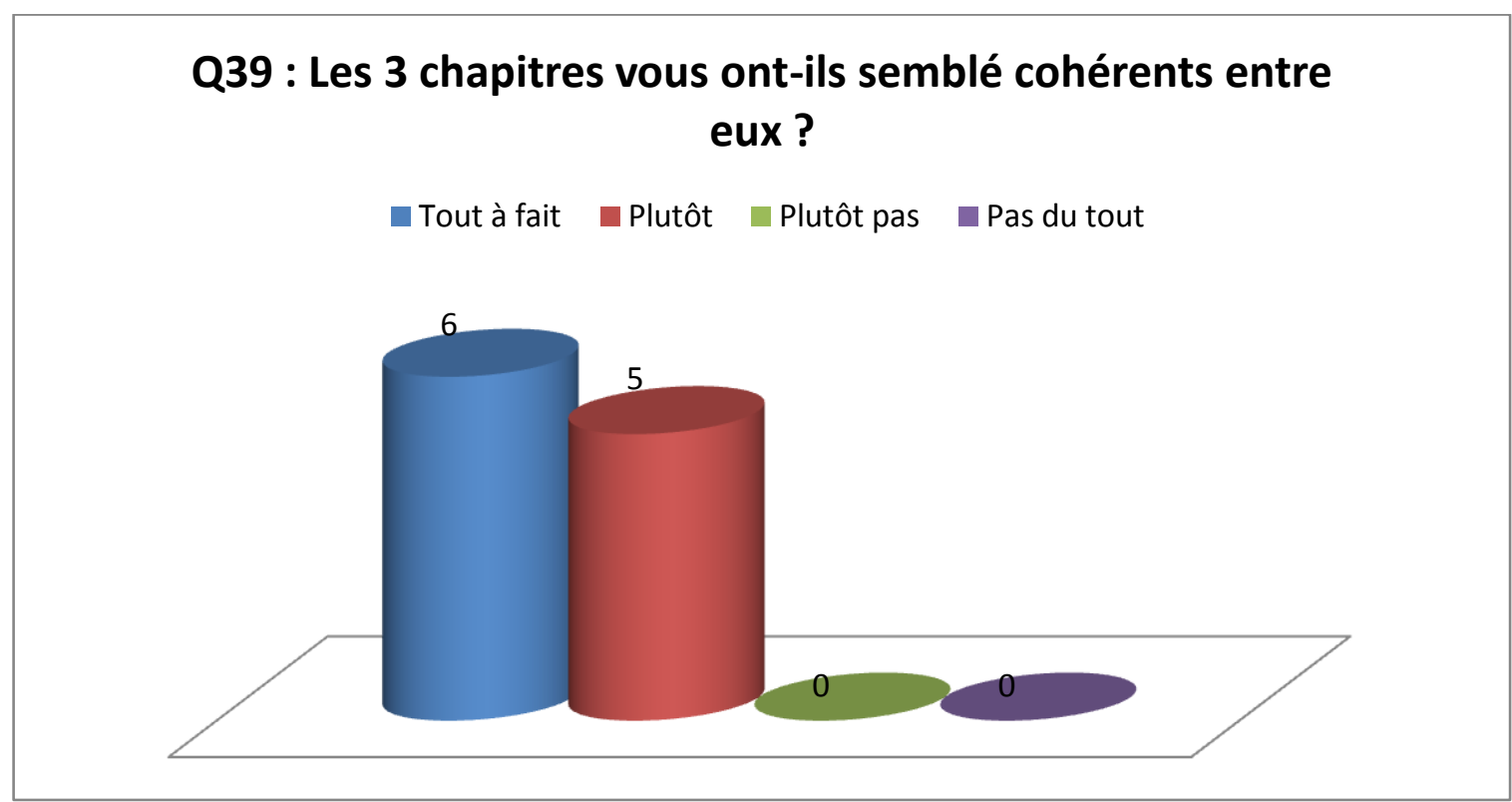

Figure 33 : Réponses à la question $\mathbf{n}^{\circ} 39$

6 patients $(54,5 \%)$ ont estimé que les trois chapitres disponibles étaient tout à fait cohérents entre eux et 5 patients $(45,5 \%)$ ont estimé qu'ils étaient plutôt cohérents entre eux. 
Au total, les patients interrogés ont estimé que le site INDIGO avait répondu à leurs besoins. Ils ont considéré que les chapitres étaient cohérents entre eux.

L'analyse des entretiens semi-dirigés nous a apporté des détails concernant l'appréciation d'ensemble du site INDIGO par les patients interrogés.

Les patients ont déclaré avoir aimé parcourir le site INDIGO : "j'ai été agréablement surprise » (1), «je sais que j’y ai passé du temps mais je ne me suis pas ennuyée devant» (3) ; «j'ai beaucoup apprécié » (5) ; «je l'ai trouvé bien » (6) ; "il m'a vraiment plu» (9) ; « on ne s'ennuie pas quand on regarde le site » (10).

Les patients ont considéré que les chapitres étaient cohérents entre eux. Malgré tout trois patients ont noté des répétitions entre le chapitre 1 «Mes objectifs glycémiques » et le chapitre 2 «Mes hypos, mes hypers »: «quand on lance le chapitre 2 on nous propose de choisir les sous-chapitres et il y en avait un il me semblait que ça faisait un peu redondant avec le chapitre précédent » (2) ; «les chapitres 1 et 2 j'ai l'impression qu'il y a certaines informations qui se recoupent, si on enchaîne les trois ça peut faire un peu répétition » (8); "le deuxième chapitre répétait certaines choses du premier, ça m'a moins apporté de choses, j'ai passé un peu directement le début du deuxième chapitre parce que ça reprenait vraiment «Mes objectifs glycémiques » (9).

Voici ce que les patients n'ayant pas bénéficié d'ETP antérieure ont dit avoir retiré du site INDIGO : «tous les petits réglages, les petits détails qui peuvent servir au quotidien ça pourra être pratique » (1); "le site a déjà répondu à beaucoup de questions, c'est très utile pour moi qui vient de découvrir le diabète, je suis en train d'apprendre et ça m'a beaucoup aidé ; il m'a apporté des confirmations de tout ce que j'ai appris à l'hôpital » (4) ; « ce que j'ai aimé c'est tout cet apport d'informations qui me permettent de faire les bons gestes parce que c'est ça le plus important pour moi : est-ce que je savais bien maitriser mon dosage d'insuline, utiliser le stylo ? ; c'est un outil tout à fait adapté à moi, j'en ai encore besoin pour m'aider même si je fais les choses correctement d'après la diabétologue; au début je l'ai géré avec un minimum d'infos, aujourd'hui j'en ai un peu plus et je peux passer à une autre étape pour me saisir de mon diabète » (6); "il n'y a rien où je me sois dit «ça on ne me l'a pas dit », c'était plus un rappel en fait; mais ça ne fait pas de mal de revoir les infos une seconde fois; je m'en étais souvenu mais je 
comprends que ça puisse être utile si quelqu'un n'intègre pas, parce que c'est vrai que quand on est hospitalisé au début il y a beaucoup d'infos » (8) ; "ça m'a appris pas mal de choses vu que mon diabète est récent, il y a des aspects de la maladie sur lesquels je ne m'étais pas encore penchée; c'est bénéfique pour mon quotidien; ça m'a permis de relativiser $\gg(9)$.

Voici ce que les patients ayant bénéficié d'une ETP antérieure ont dit avoir retiré du site INDIGO : "j'en connaissais quand même une bonne partie vu que j'ai fait le stage mais des rappels ça ne fait pas de mal; ça ne fait pas de mal de voir le site quand on a déjà eu une éducation thérapeutique, c'est peut-être un peu répétitif mais ça permet de prendre du recul, du temps pour soi, pour s'écouter; on apprend quand même pas mal de choses, la grille d'analyse des erreurs je ne connaissais pas ; ça permet de rappeler les bases d'être diabétique, toutes les problématiques autour » (2) ; "j'avais globalement déjà tout vu et j'avais bien compris, entre temps j'avais un peu oublié en ne retenant que l'essentiel ; ça m'a permis de faire un petit point et de refixer les choses que j'avais déjà globalement comprises avant et aussi de voir que je ne ressentais pas aussi bien mes hypos que je le pensais » (5); «en toute objectivité je pense qu'on m'avait quasiment tout dit, je n'avais pas forcément tout retenu ça c'est sûr ; le chapitre "hyperglycémie et acétone » comme ça ne m'est pas encore arrivé, ça m'a permis de me remémorer ce qu'on m'avait dit à l'hôpital sur ce qu'il fallait faire, ce qu'il se passait quand on manquait d'insuline ; ça permet de se rassurer et d'être sûre qu'on n'est pas dans le faux par rapport à ce qu'on croit savoir; je pense que ça manquait» (7) ; "j'ai trouvé que c'était bien parce que c'était un rappel de ce que j'avais vu en insulinothérapie ; ça m'a rappelé deux ou trois choses qui me semblaient importantes et dont je ne me rappelais plus; ça permet de mieux comprendre et ça répond à beaucoup de questions et plus on connait, moins on est stressé »(10). 


\section{Analyse des appréciations chapitre par chapitre à partir des entretiens semi-dirigés}

L'analyse qui suit est une analyse des réponses obtenues de manière spontanée ou en réponse aux questions ouvertes «Qu'est-ce qui vous a plu dans ce chapitre ? », «Qu'est-ce qui vous a moins plu dans ce chapitre ? », «Les informations contenues dans ce chapitre ont-elles répondu aux questions que vous vous posiez?», «Avez-vous des suggestions d'amélioration concernant ce chapitre ? » qui ont été posées à chaque patient pour chacun des trois chapitres. (cf. Guide d'entretien en Annexe 6)

\subsection{Chapitre 1 : «Mes objectifs glycémiques »}

\subsubsection{Les points positifs}

\subsubsection{Les témoignages $(6 / 11)$}

Six patients ont déclaré que les témoignages leur avaient particulièrement plu dans le premier chapitre.

Ils ont apprécié la diversité des points de vue : «confronter les deux points de vue entre un jeune en quelque sorte et une personne un peu plus adulte, ça j'ai bien apprécié » (2); «ce que j'ai trouvé bien c'est que tout le monde n'a pas la même manière de percevoir sa façon de vivre et sa... enfin sa moyenne, là où il veut se situer » (10); la possibilité d'identification : «j'ai aimé trouver toutes ces infos qui correspondent un peu à ce que je vis, ça correspond à comment je vis mon diabète » (6) ; "j'ai trouvé que c'était pas mal aussi parce que bon c'est vrai qu'on s'identifie aussi aux gens de la vie de tous les jours » (10) ; et la confrontation aux autres : « déjà ça m'a permis de voir que je n'étais pas tout seul dans ce cas-là. Donc déjà ça c'est un premier point. Et puis ça m'a permis aussi de voir un peu comment les gens vivaient avec et donc moi essayer de comparer mon cas par rapport à ce que eux présentaient » (3).

Un des patients a aimé leur agencement : «moi, j’ai bien aimé le $1^{\text {er }}$ chapitre avec l'enchainement des témoignages qui sont bien structurés, qui passent étape par étape» (2). 


\subsubsection{Les objectifs glycémiques $(6 / 11)$}

Deux patients ont été satisfaits de trouver des objectifs glycémiques détaillés en fonction des différentes situations de la vie quotidienne : «c'est dans celui-là où j'ai appris que quand on prend la voiture il vaut mieux être au-dessus de 1.20, quand on fait du sport il vaut mieux partir un peu plus élevé ou que 2 heures après le repas il faut être à peu près à 1.80 et à 3 heures à 1.60. C'est vrai que c'est des repères, ça a recadré un petit peu les choses » (3); " il y a aussi quelques détails en plus sur des cas, comme quand on fait des activités sportives ou quand on mange plus, moins. Ça m'a apporté des informations un peu plus détaillées peut-être que ce que j'avais retenu de toutes les discussions qu'on avait eu. C'est très utile» (4).

Deux patients ont particulièrement apprécié le schéma : «le compteur de la glycémie » (cf. Annexe 2) : "j'ai aimé le récapitulatif à la fin des explications, le schéma avec le petit disque pour bien visualiser en fait, se rendre compte des glycémies. Là tout de suite je pense à l'explication : entre telle et telle glycémie évitez de conduire... » (9).

Trois patientes ont été satisfaites de constater qu'elles pouvaient envisager des objectifs glycémiques moins stricts : "je n'avais pas spécialement intégré ou compris le fait que chacun devait choisir dans quelle tranche il voulait se mettre. J'avais bien compris les tranches où il ne fallait pas être, celles où c'était le plus normal d'être et je pensais qu'il fallait à tout prix que je sois dans cette tranche-là. Mais à la suite de ça, je me suis rendu compte que ce n'était pas ce qui me convenait et que sans risque pour ma santé, je pouvais décaler cette tranche. Ça a été un petit soulagement. Ça met une certaine pression de se dire qu'il faut être entre 0,70 et 1,20 avant de manger, c'est compliqué à gérer en fait. Savoir que l'on peut se permettre des écarts, enfin pas des écarts mais une tranche plus large, ça sérénise un peu» (1); "je ne pensais pas que ça pouvait être autant individualisé les résultats glycémiques. Pour moi il y avait une tranche à respecter; c'était pour être bien entre 0,90 et 1,20; et en sortant de ça, ça n'allait plus. Mais c'est vrai que j'essayais parfois de trop respecter ça et je me trouvais beaucoup trop en hypo dès que je sortais. Là ça devenait ma hantise ces derniers temps de devoir me resucrer ou de m'asseoir en pleine rue. Ça m'a permis de relativiser : oui je suis peut-être à 1,50 avant d'aller courir mais il vaut mieux. Là ça fait une semaine que je n'ai pas fait d'hypoglycémie. Maintenant ça m'arrive d'être plus vers 1,60 ou 1,70 mais je ne me fais pas d'injection, je sais qu'à ce moment-là je vais sortir et que ça va baisser tout seul et que 
je ne suis pas obligée de m’injecter à chaque fois» (9); «je me suis dit: oui, effectivement, on n'est pas obligés d'être à 0,80 ou à 1,20 pour être toujours super bien » (10).

\subsubsection{L’interactivité (4/11)}

Deux patientes ont aimé pouvoir inscrire leurs propres données : «j’ai bien aimé que l'on puisse participer en disant nos glycémies... enfin ce que nous on souhaite voir sur l'écran, que l'on note tout ça, que l'on voit où on en est. Ça permet de faire un point » (5) ; "j'ai aimé le fait qu'il y ait autant de participation et que l'on puisse à la fin avoir un récapitulatif de ses objectifs personnels de glycémie »(9).

Deux patients ont apprécié en particulier la séquence « Regardez vos 50 dernières mesures » : "ce qui était intéressant, c'est qu'il fallait dire combien de fois on avait été en hypoglycémie sur les cinquante derniers résultats et aussi combien de fois on avait été en hyperglycémie. Donc ça c'était pas mal, ça permet de faire un petit point» (2); "les cinquante mesures ça peut-être pas mal, avoir un compte-rendu par ça, pourquoi pas » (8).

\subsubsection{Autres réponses}

Une des patientes a apprécié le point de vue médical : "j'ai aimé avoir le conseil de plusieurs médecins dont le Pr BENHAMOU» (5).

Une autre a été satisfaite de trouver des rappels sur les horaires pour pratiquer sa glycémie postprandiale et sur la variabilité naturelle de la glycémie : "la glycémie capillaire j'avais tendance peut-être à la faire trop tôt après le repas donc ça m'a rappelé que c'est 2 à 3 heures. Souvent j'avais des résultats un peu trop élevés. Et puis aussi de savoir qu'un non diabétique a des fluctuations aussi au niveau de sa glycémie. Parce que je me disais monter aussi haut... enfin ça me faisait peur. De me dire le temps que l'insuline agisse je suis exposée au risque. Les fluctuations sont valables pour les non diabétiques en fait, le temps que le pancréas fasse son travail »(9). Elle a également apprécié les informations sur l'hémoglobine glyquée : "ça m'a un peu permis de comprendre comment ça fonctionnait, comment on établissait les résultats sur trois mois » (9). 


\subsubsection{Les points négatifs $(3 / 11)$}

La patiente $\mathrm{n}^{\circ} 7$ a été troublée par l'un des témoignages : "il y a un témoin qui disait qu'elle ne voulait jamais être au-dessus de 1. Ça si je l'avais entendu il y a quelques mois ça m'aurait vraiment fait peur. Le fait que l'on montre plusieurs personnes qui ont des objectifs différents c'est très important, ça rassure. Mais en même temps, comme on veut essayer généralement de faire le mieux possible on va plutôt retenir la personne qui est la plus exigeante dans ses objectifs glycémiques. Enfin c'est ce que j'ai retenu, et du coup moi je me suis dit "Je ne suis peut-être pas assez rigoureuse dans mes objectifs glycémiques. »Parce que pour moi l'idéal ce n'est pas d'être forcément entre 1,20 et 1,40 tout le temps. J'avoue que je l'ai regardé assez rapidement pour éviter de me retrouver face à une situation où je vais me dire "Bon ben ça va pas très bien en fait, je ne suis pas assez rigoureuse. " Même si mes hémoglobines glyquées sont correctes, il n'empêche que j'ai un peu le sentiment que je peux mieux faire en fait. Ce chapitre je ne l'ai pas regardé en entier, il m'a mise mal à l'aise. Mais peut-être encore une fois parce que je suis dans un diabète qui est encore assez récent je ne sais pas. »

Le patient $\mathrm{n}^{\circ} 8$ a trouvé ce chapitre peu utile pour lui : "depuis ma sortie j'ai intégré les taux. Et puis je vous dis honnêtement dans le service j'étais super bien suivi donc au niveau des taux, quoi faire... Les vidéos, je les regardais, mais parfois c'était un tout petit peu long, du coup je zappais. Et c'est vrai que je m'arrêtais peut-être plus sur ce qui était écrit, les schémas etc. Le $1^{\text {er }}$ chapitre il est assez... en fait il ne sert pas trop quand on a été bien briefé à l'hôpital. »

Idem pour la patiente $\mathrm{n}^{\circ} 11$ : «moi en fait on $m^{\prime}$ a déjà tout bien expliqué avant donc c'est peut-être ça qui fait que j'y ai prêté moins attention, je ne sais pas. Voilà mais pour quelqu'un qui ne connait pas, c'est très bien expliqué. »

De plus elle a noté certaines répétitions dans le chapitre : «après c'était un petit peu long, enfin j'ai trouvé que ça se répétait un peu parce qu'il y a un moment le Pr BENHAMOU explique qu'il faut être entre tant et tant ou tant et tant et en fait juste après on répète, il y a à nouveau un schéma où on redit la même chose et j'ai trouvé que ce n'était pas utile. Soit on met l'un, soit on met l'autre, mais mettre deux séquences où on dit la même chose j'ai trouvé que c'était un peu répétitif.» 


\subsubsection{Les informations ont-elles répondu aux questions des patients interrogés concernant leurs objectifs glycémiques ?}

Sept patients ont répondu «oui » (les patients $n^{\circ} 1,2,3,4,5,9$ et 10). La patiente ${ }^{\circ} 7$, qui a été troublée par ce chapitre et ne l'a pas regardé en entier, a répondu «pas vraiment». Deux patients comme nous l'avons vu connaissaient déjà leurs objectifs glycémiques (les patients $n^{\circ} 8$ et 11 ). Par oubli, la question n’a pas été posée à la patiente $n^{\circ} 6$.

\subsubsection{Suggestions d'amélioration}

Le patient $\mathrm{n}^{\circ} 2$ aurait souhaité avoir des informations sur les témoins : «il manquait des infos sur les témoins, savoir si ce sont de vrais diabétiques, depuis combien de temps; si c'est récent ou plutôt ancien; pour voir si c'est un nouveau ou si il a de l'expérience, à quel stade il en est, ça aurait été pas mal »(2).

La patiente $\mathrm{n}^{\circ} 11$ a évoqué le fait de ne garder que l'interview du Pr BENHAMOU et de retirer le schéma «le compteur de la glycémie » en raison de la redondance de leurs contenus. Deux patients avaient quant à eux souligné leur intérêt pour ce schéma. 


\subsection{Chapitre 2 : «Mes hypos, mes hypers »}

\subsubsection{Les points positifs}

\subsubsection{Chapitre 2.1 : «Comment savoir où j'en suis au niveau de ma glycémie ?» $(8 / 11)$}

Les patients ont apprécié la partie «Quels sont mes symptômes d'hypo et d'hyper ? ». Ils ont aimé pouvoir répertorier leurs symptômes d'hypoglycémie et d'hyperglycémie dans les listes proposées : «il y a toute la liste des symptômes connus par exemple. Oui ça aide bien sûr, ça colle bien avec les symptômes que j'ai, pas tous mais la plupart oui. Au moins pour l'hypoglycémie, pour l'hyperglycémie honnêtement je n'en ai pas ou très rarement $\gg(4)$.

Cela leur a permis de préciser leurs ressentis d'hypoglycémie et d'hyperglycémie : «ça m'a permis de bien visualiser tout ce qui était sensations pour les hypos et les hypers et de voir que même si cela se ressemble, pour moi, dans mon ressenti, il y a une grande différence. Bien mettre un mot sur la sensation en fait. Ça va me permettre de plus faire attention. D'avoir mis un mot, de l'avoir noté : telle sensation il faut faire attention à ça, à ça, à ça... de l'avoir listé en fait, c'est plus clair dans ma tête » (1); "j'ai trouvé que c'était pas mal aussi parce que justement ça permet d'analyser un peu comment on se sent. On ne sait pas toujours comment on se sent... C'est vrai qu'il y a aussi des moments où les hypers et les hypos ça peut se ressembler. C'est pour ça qu'après affiner sa recherche j'ai trouvé que c'était pas mal »(10).

Ils ont souligné l'importance de pouvoir y revenir et de pouvoir l'imprimer : «c'est écrit quelque part, donc je peux y retourner si j'ai un doute. C'est plus visuel. Et pareil pour les hypers du coup » (1); "le fait que l'on revienne dessus parce qu'ils nous rappellent ce qu'on a mis en fait, ce qu'on a coché justement et pouvoir l'imprimer je pense que c'est un point positif pour pouvoir en discuter avec sa diabéto »(10).

Ils ont ensuite aimé « le carnet INDIGO » (cf. Annexe 9), outil qui leur permet de noter leurs symptômes, les «indices extérieurs» (alimentation, activité physique...), leur glycémie estimée et la glycémie mesurée. Cette démarche a été estimée formatrice : «le carnet INDIGO ça peut être pas mal pour une personne qui a un diabète qui n'est pas très bien équilibré, pour faire un point, bien comprendre, expliquer les glycémies élevées ou basses. Parfois c'est un peu dur de l'expliquer donc avoir cet outil-là dans ce genre de situation ça peut être bénéfique je pense » (2) ; "le carnet INDIGO je ne m'en suis par 
servie techniquement, je ne l'ai pas pris pour support. Mais l'idée d'estimer ses glycémies je commence à le faire, c'est-à-dire avant de me piquer de me dire : "Là tu penserais que tu es à combien? " et de vérifier. J'ai souvenir d'une fois où je me croyais vraiment en hypo et j'étais plutôt haute. L'inverse aussi est arrivé. Ça m'a permis de voir en fait que je ne sentais pas forcément aussi bien que je le pensais » (5) ; "pour moi le carnet INDIGO je trouve que c'était pas mal parce que de bien noter finalement les choses qu'on ressent je trouve que ça nous aide à mieux comprendre les choses aussi. On ne nous en avait pas parlé je trouve ça super parce que c'est vrai que le fait de vraiment essayer d'analyser ce qu'on ressent, je trouve qu'on est plus à l'écoute et du coup c'est plus fiable en fait. On a plus l'impression de savoir ce qu'on ressent. Et du coup, de mieux analyser, ça me permet de me sentir tout de suite plus en alerte. Moi ça m'aidera je pense » (10).

\subsubsection{Chapitre 2.4 : La grille d'analyse des erreurs (8/11)}

Cet outil (cf. Annexe 8) permet aux patients d'évaluer la marge d'erreur entre la glycémie qu'ils estiment en se basant sur leurs ressentis et la glycémie qu'ils mesurent et de savoir dans quel sens les erreurs sont faites. Le but est d'être de plus en plus précis dans ses estimations en comprenant quelles erreurs sont faites.

Les patients l'ont jugée utile et enrichissante pour faire le point sur le ressenti de leurs glycémies : "j’ai trouvé ça intéressant parce que je ne l'avais jamais vue. Je vais l'imprimer. Après pas le faire tous les jours mais le faire pendant deux semaines pour voir à peu près, parce que ça permet de faire un point, de prendre un peu de recul. Du coup c'est pas mal pour se consacrer un peu de temps à soi-même parce qu'avant de faire la glycémie on n'estime pas vraiment à combien on va être. Donc là ça permet au moins de se poser, peut-être se concentrer trente secondes pour dire : "Tiens, comment je me sens? Est-ce que je me sens plutôt bien ou plutôt mal ?» (2) ; "la grille d'analyse des erreurs j'ai bien aimé. C'est très bien parce que ça permet de faire la corrélation entre vraiment ce qui est mesuré donc ce qui est objectif et son ressenti. Et puis je suis scientifique, donc forcément j'ai été un peu plus sur ce qui est plus cartésien on va dire, plus chiffrable. Donc ça m'a plu. Je ne l'ai pas encore fait, mais il va falloir que je m'y mette. Je pense que ça va m'aider. Voir à peu près si je suis à $5 \%$, à $20 \%$ ou carrément hors zone, si vraiment je suis à côté de la plaque »(3).

Une patiente a considéré la grille comme un outil complémentaire en cas de déséquilibre : «je pense que si à un moment donné mon diabète se déstabilise un peu je vais vraiment me servir de ces outils-là pour remettre un peu les choses d'équerre. Par 
exemple, là je dois faire une hémoglobine glyquée. Si je ne suis plus dans les clous je pense que je vais approfondir ça»(7).

Elle a réconforté une patiente quant à ses ressentis : "la grille d'analyse des erreurs c'est très pratique, je l'ai imprimée. J'ai commencé, alors je ne le fais pas à chaque fois, j'attends d'avoir un petit bout de liste et de le marquer. Au début ça m'inquiétait de voir que le chiffre que j'allais me dire dans ma tête ne correspondait pas à celui que je voyais dans mon lecteur et au final en fait j'étais plutôt dans la bonne partie de la grille, j'étais plus dans le vert que dans les autres couleurs. Donc du coup ça rassure aussi » (1).

Enfin deux patientes l'ont trouvée intéressante mais en ont souligné le caractère contraignant : "j'aimerais bien utiliser la grille d'analyse des erreurs mais comme c'est assez contraignant je pense que je vais le faire comme ils disaient, c'est-à-dire une petite semaine ou un moment bien, mais je ne pourrai pas le faire tout le temps. Mais ça m’intéresse » (5) ; «je pense que ça serait bien, honnêtement. Mais comme il le dit dans la vidéo, ça prend beaucoup de temps et c'est super contraignant d'écrire à chaque fois. Je ne le ferai pas, honnêtement. Mais je pense que c'est très bien par contre »(11).

\subsubsection{Les témoignages $(4 / 11)$}

Un patient a estimé qu'ils favorisaient l'apprentissage : "les témoignages, ça m'a aidé vraiment à retenir les informations. Le fait que ce soit dit par quelqu'un : "Moi, je fais ça et ça et ça » et après il y a le commentaire derrière qui dit «Oui c'est bon de faire ça » ou «Non ce n'est pas correct de faire ça»»(4).

Une fois encore, la variété des témoignages a été mise en avant : «dans la pratique vu qu'ils ne font pas tous la même chose c'est vrai qu'on se reconnait un peu. C'est la diversité des exemples m'a plu» (5); "ce qui m'a plu ce sont les témoignages moi, c'est surtout ça. C'est important d'avoir un panel pour moi, parce qu'au début je pensais qu'on était tous pareils à gérer notre diabète alors que depuis le début on me dit «ça appartient à chacun ». Et là les témoignages ont permis de balayer cette idée que j'avais, bien que j'avais eu cette info par l'équipe médicale, mais elle était ancrée quand même »(6). 


\subsubsection{Chapitre 2.3: « J'ai des problèmes d'hypos sévères » (3/11)}

Les patients ont apprécié de voir comment utiliser le Glucagon dans la réalité de l'urgence : "la partie sur l'hypo sévère était bien aussi. J'en avais parlé avec le Pr BENHAMOU et c'est vrai que c'était pas mal de voir comment le Glucagon ça se fait en vrai » (8) ; «on ne m'avait pas vraiment expliqué comment utiliser ce produit, dans quel cas vraiment, parce que ça m'arrive de faire des hypos et d'être vraiment très basse. Pour moi c'était vraiment quelque chose d'abstrait, que l'on n'avait pas forcément à avoir chez soi » (9) ; "le fait d'expliquer comme ça à l'oral, bon, on se dit on comprend. Mais le fait de le voir c'est vrai que c'est beaucoup plus rassurant pour les gens. Savoir comment on utilisait vraiment le produit, comment on l'injectait etc. C'est plus parlant de le voir en fait $\gg(10)$.

Un patient a été satisfait de trouver un complément d'information sur le délai d'action du Glucagon : "les infos on me les avait déjà données, par contre il y a quelque chose que je ne savais pas, c'était la durée d'action. C'est vrai que c'est peut-être pas mal d'informer l'entourage. Parce que, bon j'ai informé mon entourage en disant : "En cas de pépin il faut faire ci, il faut faire ça " mais c'est vrai que d'informer sur le fait que ça puisse durer dix minutes un quart d'heure avant que l'on reprenne conscience ça peut être pas mal parce que bon, ça évite à la personne d'être en stress pendant dix minutes pour rien $\gg(8)$.

Ils ont estimé que cette séquence devait être vue par leur entourage : «c'est peut-être la séquence la plus parlante pour l'entourage» (8); "j'aurais aimé que mon copain le regarde avec moi » (9) ; " tout ce qui est Glucagon tout ça, ça j'ai trouvé que c'était super parce que ça a vachement rassuré ma mère ; et ma sœur m'a dit que le fait de le voir, c'est un peu dédramatisant parce qu'on se dit que ce n'est pas bien compliqué. J'ai trouvé que c'était bien et pour mon entourage et pour moi » (10).

\subsubsection{La notion de baisse du seuil de ressenti des hypoglycémies}

\section{$(2 / 11)$}

Deux patients ont trouvé utile d'apprendre cette notion : «le fait qu'à un certain point, si on ne se resucre pas quand on descend à un certain niveau, si on se resucre trop tard, notre corps va s'adapter et donc on ne va pas les ressentir» (4); "la partie qui était la plus intéressante je trouve c'était celle où c était précisé que le cerveau se déshabituait du signal des hypos si on ne les traitait pas en-dessous de 0,7 par exemple et puis après on pouvait aller à jusqu'à 0,6 puis 0,5 puis 0,4. Ça c'était intéressant je trouve. C'est pas mal, 
ça motive à dire en-dessous de 0,7 tout de suite je fais quelque chose. Et le fait que ça soit réversible c'était pas mal aussi d'informer là-dessus. Même si on ressent très bas, on peut faire remonter le moment déclencheur pour que le cerveau sache. Ça c'était intéressant» (8).

\subsubsection{Les points négatifs}

\subsubsection{Chapitre $2.3:$ « J'ai des problèmes d'hypos sévères » $(5 / 11)$}

Deux patients ne se sont pas sentis concernés : "la partie de l'hypoglycémie sévère c'est bien mais ça ne s'applique pas encore à moi et j'espère que cela ne s'appliquera jamais. Pour ma situation à moi, c'est peut-être un peu trop long » (4) ; "je n'ai pas fait « J'ai des problèmes d'hypos sévères ». J'ai vu le titre et j'ai sélectionné parce que je ne suis pas concerné » (3).

Trois patients ont regretté la mise en scène de la vidéo : "ce qui m'a fait un peu rire c'était la scène quand la personne s'endort devant la télé, ça m'a fait un peu sourire, c'est un peu sur joué. Après c'est normal, ce ne sont pas des acteurs, c'est ça qui est marrant. Peut-être que c'était un peu trop accentué sur le fait qu'il soit endormi devant la télé et puis qu'après elle le secoue. Ça prenait peut-être un peu de temps, je veux dire par rapport au but que ça visait au final. Après le temps d'expliquer ce qu'il faut faire et tout ça prenait le bon temps » (2) ; «je me suis demandée si c'est vraiment nécessaire de faire de grandes grandes mises en scène » (6) ; "les vidéos c'est vraiment... enfin moi ça me faisait rigoler parce que je trouvais que c'était très mal joué, mais est-ce que c'est ça qu'il faut pour que les gens comprennent bien comment ça se passe? Je ne sais pas mais il y a des vidéos je trouvais ça vraiment... je ne sais pas. Par exemple pour le Glucagon... Ça m'a fait plus sourire qu'autre chose, mais au final on regarde quand même. Après peutêtre que j'étais moins sérieuse » (11).

\subsubsection{Chapitre 2.1 : «Comment savoir où j’en suis au niveau de ma glycémie ?» $(2 / 11)$}

Une patiente n'a pas trouvé instructive la partie «Quels sont mes symptômes d'hypo et d'hyper »: «il y a des questions auxquelles je n'ai pas répondu, notamment pour les hypers et les hypos, quand ils demandent les sensations tout ça. C'étaient des choses, par exemple comment on se sent quand on est en hypo, en hyper, enfin on le vit donc du coup on sait ce qu'on ressent voilà. Pour moi ça ne m'apportait rien » (11). 
Deux patients ont regretté la qualité de jeu d'acteur de la vidéo «Quel temps accordé pour remplir le carnet INDIGO dans ma vie quotidienne ? » : il y avait une scène qui était un peu sur jouée. C'était le cas du jeune qui balance son carnet et qui crie «Oui,j'en ai marre de ce diabète ». Ça m'a fait rire » (3) ; "la vidéo du jeune homme elle m'a fait un petit peu sourire, mais c'est quand même le reflet de la réalité » (11).

\subsubsection{Chapitre 2.4 : La grille d'analyse des erreurs (1/11)}

Une patiente a déclaré que ce graphique ne lui convenait pas : «la grille d'analyse des erreurs je l'ai trouvée bien mais moins parlante pour moi, le graphique comme ça... J'ai compris le fonctionnement général mais je ne suis pas sûre que j'arriverai à l'utiliser toute seule. Non je pense que c'est vraiment une question de personnalité, moi je sais que le graphique, même amélioré, je pense que je ne l'utiliserai pas »(10).

\subsubsection{Les témoignages $(1 / 11)$}

Une patiente a regretté la place trop importante des témoignages dans ce chapitre au détriment de l'interactivité : «les témoignages étaient plus longs. Il y avait beaucoup plus de vidéos donc il y avait moins de participation que dans le $1^{\text {er }}$ chapitre. Je l'ai trouvé moins léger que le $1^{\text {er }}$ chapitre, c'est ce qui m'a poussée à avancer plus vite dans le chapitre » (9).

\subsubsection{Les informations ont-elles répondu aux questions des patients} interrogés concernant leurs hypoglycémies et leurs hyperglycémies ?

Sept patients ont répondu «oui » (les patients $n^{\circ} 1,3,4,6,7,8$ et 10). La patiente $n^{\circ} 5$ a répondu "peut-être sur la façon de traiter les hypers par contre pour les hypos ça va j'étais au point je pense». Les patients $n^{\circ} 2$ et 9 ont répondu «oui, surtout pour les hypos ». La patiente $\mathrm{n}^{\circ} 11$ a répondu « je le savais déjà, ça ne m’a pas apporté grand-chose».

\subsubsection{Suggestions d'amélioration}

Le patient $\mathrm{n}^{\circ} 2$ a suggéré de modifier la vidéo « J'ai des problèmes d'hypos sévères » : "c'était peut-être disproportionné le temps de la découverte qu'il est en hypoglycémie, c'était trop accentué sur le début de cette scène avant que ça commence réellement et qu'on voit comment faire le glucagon. »

Il aurait également aimé avoir plus d'informations sur les hyperglycémies : «le chapitre 2, je n'ai pas le souvenir qu'il traite beaucoup des hypers. Peut-être que j'aurais préféré, 
en amélioration, qu'il parle aussi un peu plus des hyperglycémies. Moi, je sais quoi faire mais après pour une personne qui n'est pas au courant. De mémoire comme ça, ils insistaient beaucoup sur les hypoglycémies avec l'injection du Glucagon mais de mémoire... enfin les hypers ça m'a un peu moins marqué. Après je ne sais pas si vraiment ça y était ou pas. »

La patiente $n^{\circ} 9$ a suggéré l'ajout de quelques schémas récapitulatifs comme elle avait trouvé dans le chapitre 1.

La patiente $\mathrm{n}^{\circ} 10$ aurait souhaité pouvoir imprimer le carnet INDIGO au format A4. 


\subsection{Chapitre 3 : «Mes insulines »}

Seuls neuf patients avaient visionné ce chapitre. (cf. 4. Modalités d'utilisation)

\subsubsection{Les points positifs}

\subsubsection{Chapitre 3.1: «Comprendre mon traitement par insuline »}

Trois patients ont aimé en apprendre plus sur les insulines, leur rôle, leur action, leur cinétique etc. : « refaire un point, comprendre comment marche ce que l'on s'injecte tous les jours. De savoir quand l'insuline est active, quand elle l'est moins ça permet aussi de comprendre ses glycémies, de savoir quand les regarder, ce qu'elles signifient et à quel moment» (5) ; "l'insuline c'est vrai que c'est pas mal de le revoir, de refaire le point sur la durée d'action etc. et ce qui m'a bien intéressé c'était le schéma du corps humain avec sur quels organes agissait l'insuline, dans quel ordre, dans quelle situation etc. Ça c'était bien parce qu'on me l'avait fait à l'hôpital mais vu que c'est assez complexe c'était pas mal de le revoir» (8); "ce que j'ai bien aimé c'est quand ils ont expliqué le fait que l'insuline elle est très active je crois de 30 à 70 minutes. Elle a un pic d'action qui est plus important et puis après ça redescend et aussi l'information selon laquelle la température du corps, la chaleur, ça peut faire varier aussi la glycémie. Parce que moi par exemple quand je me douche, si je mets de l'eau chaude, d'un seul coup je peux vite me retrouver en hypo alors que je viens de manger il y a moins d'une heure. Je me disais "Mais pourquoi? », après je me disais : "Est-ce que je ne me suis pas fait la bonne dose d'insuline? »alors que je pèse tous mes aliments donc je fais super attention. Je pense que plus on connait, moins on est stressé. Si on sait qu'on a mangé peut-être plus léger, peutêtre qu'on préfère se doucher le lendemain. Ça me permet de mieux comprendre et je pense que le fait de mettre des mots sur ses sensations c'est hyper important je pense, on doit dédramatiser aussi parce qu'on comprend mieux »(10).

\subsubsection{Chapitre 3.3 : «Comment définir mes doses d'insuline » (3/9)}

Trois patients ont aimé le calcul des doses de correction : " tout ce qui était calcul théorique pour les doses de rattrapage jusqu'ici je le faisais un petit peu en me disant «Je mets 1, 2, 4... » Mais ça aussi à partir du moment où j'aurai bien intégré le calcul, ça pourra être très pratique » (1) ; "il m'a aussi donné des informations que je n'avais pas, comme corriger les valeurs de glycémie, s'il faut et quand il faut le faire. C'était bien utile, 
bien intéressant d'avoir cette information de plus » (4); «il y a des choses que moi j'ai apprises au fil des mois alors que là toutes les infos sont condensées. Notamment pour traiter les hypers, je trouve que c'est très bien fait avec calculer sa dose d'insuline pour faire descendre en fonction de... enfin la dose de rattrapage, ça je sais que j'ai mis un petit temps à être à l'aise avec cette question-là » (7).

\subsubsection{Chapitre $3.4:$ «Le manque d'insuline : hyperglycémie et acétone $\gg(3 / 9)$}

Trois patients ont été satisfaits de trouver des informations pour les aider à gérer cette situation : "on explique bien que ce n'est pas bon, qu'il faut l'éviter et comment l'éviter. On explique bien toutes les procédures »(1).

Même si pour deux d'entre eux, il s'agissait de rappels : "ça m'a permis, comme ça ne m'est pas encore arrivé, de me remémorer ce que l'on m'avait dit à l'hôpital sur ce qu'il fallait faire quand on manquait d'insuline » (7) ; "j'ai trouvé que c'était bien parce que c'était un rappel de ce que j'avais vu en insulinothérapie : comment réagir quand on a de l'acétone parce que c'est vrai que bon ça m'est arrivée d'être en hyper et l'acétone je l'ai pas fait »(10).

La patiente $\mathrm{n}^{\circ} 10$ a par ailleurs aimé le fait que les consignes à respecter en cas d'acétonurie soient imprimables.

\subsubsection{4. $\quad$ Autres réponses}

La patiente $\mathrm{n}^{\circ} 1$ a aimé avoir des informations sur le stylo et sur la pompe : «j'ai aimé tous les parallèles qui étaient faits entre les différents traitements, entre les stylos, les pompes, savoir que l'on peut passer de l'un à l'autre sans trop de difficultés, temporairement ou pas temporairement. »

Le patient $\mathrm{n}^{\circ} 2$ a aimé le chapitre $3.2:$ «Mes injections d'insuline » pour les informations sur les sites d'injection : «c'était intéressant de voir les différentes possibilités de sites d'injection. Ça c'est pas mal à rappeler. » 


\subsubsection{Les points négatifs}

La patiente $n^{\circ} 7$ a trouvé ce chapitre plus ennuyeux et notamment les sous-chapitres 3.1 : «Comprendre mon traitement par insuline » et 3.2 : «Mes injections d'insuline».

Elle les a trouvés moins utiles pour elle qui avait bénéficié d'un stage d'ETP trois mois auparavant : "il est un peu plus ennuyeux entre guillemets que le chapitre 2. Mais plus parce que c'est plus scolaire on va dire. Les explications qui sont données ce n'est pas que je les ai trouvées longues, c'est que moi étant donné que c'est clair dans ma tête... Du coup c'est vrai que ce n'est pas quelque chose qui m'a interpellée et je ne trouvais pas ça passionnant. » Elle a nuancé ses propos : «mais ça m'a fait vraiment peur au début. Et je pense que ça aurait été intéressant d'avoir ça sous la main. En sortie de première hospitalisation. Vraiment, je pense que ce serait un super support parce qu'en fait quand on découvre le diabète de type 1 tout nous paraît complètement invraisemblable. Enfin rien n'est naturel. »

La patiente $\mathrm{n}^{\circ} 10$ a signalé avoir moins apprécié l'un des témoignages de ce chapitre : «dans ce chapitre-là je crois qu'il n'y avait pas les mêmes personnages. Il y a une jeune je crois et une autre dame. Je l'ai trouvée moins bien celle-ci, je ne sais pas pourquoi. Alors pourquoi? Non je ne sais pas, une question de feeling. Mais c'est vrai que du coup ça m'empêchait un peu d'accrocher. »

\subsubsection{Les informations ont-elles répondu aux questions des patients interrogés concernant leurs insulines ?}

Quatre patients ont répondu «oui» (les patients $\left.\mathrm{n}^{\circ} 2,5,7,8\right)$. La patiente $\mathrm{n}^{\circ} 1$ n'a pas trouvé de réponse à la question «Quels sont les risques de l'hyperglycémie avec acétonurie ?». Le patient $\mathrm{n}^{\circ} 4$ n'a pas trouvé de réponse à la question « Que faire si j'ai des hyperglycémies le soir uniquement ? ». La patiente $n^{\circ} 6$ n'a pas trouvé de réponse aux questions «Comment conserver son insuline hors de chez soi ? ». La patiente $\mathrm{n}^{\circ} 10$ n'a pas trouvé de réponse aux questions «Que faire si l'on inverse ses insulines ?» et «Que se passe-t-il au niveau de la glycémie après le pic d'action de l'insuline ? ». La patiente $\mathrm{n}^{\circ} 11$ a répondu « je le savais déjà, ça ne m’a pas apporté grand-chose ». Les patients n 3 et 9 n'ont pas vu ce chapitre. 


\subsubsection{Suggestions d'amélioration}

La patiente $\mathrm{n}^{\circ} 1$ aurait aimé avoir plus d'informations sur les risques de l'hyperglycémie avec acétonurie : "la seule chose qui me manque un peu dans celui-là c'est que je n'ai pas bien cerné les dangers de l'acétone, les risques, les problèmes que ça engendrait. »

Le patient $\mathrm{n}^{\circ} 2$ aurait souhaité voir des images de lipodystrophies : «pour moi ce qui aurait été bien c'était de voir une image montrant à quoi ressemble une lipodystrophie, enfin pour montrer la différence entre avant ou après ou montrer une personne qui n'en a pas, une personne qui en a. Parce que moi du coup je suis au courant dans le cadre de ma maladie mais pour mon amie par exemple qui ne connait pas, pour elle ça aurait été pas mal d'avoir un exemple de ce que c'est. »

La patiente $n^{\circ} 6$ aurait aimé avoir des informations sur la façon de conserver ses insulines en excursion : "savoir comment conserver la rapide et la Lantus. Là aujourd'hui je reste sur Grenoble, j'ai pris mon stylo avec moi. Parce que ça a été quand même handicapant pour moi tout cet été. Moi qui ai beaucoup tendance à partir toute la journée et bien l'été en pleine chaleur... Ça j'ai pas trouvé cet élément là-dedans. Peut-être qu'il y ait plus de petites infos comme ça dans notre quotidien. On ne sait pas où pouvoir trouver la réponse. Oui comment transporter mon stylo quand je ne suis pas à la maison? C'est des choses bêtes mais importantes pour moi. »

La patiente $\mathrm{n}^{\circ} 10$ aurait aimé avoir des explications concernant la lecture des bandelettes urinaires : «moi le problème que je rencontre avec justement l'acétone, peut-être que ça on aurait dî l'expliquer, moi je comprends rien à comment il faut lire les bandelettes urinaires. Et je sais que quand j'étais en stage d'insulinothérapie je n'étais pas la seule à ne pas savoir faire. J'ai essayé de faire une fois et je n'ai rien compris. »

La patiente $\mathrm{n}^{\circ} 11$ aurait souhaité des informations plus détaillées sur les pompes à insuline : "je n'ai pas trouvé que c'était très complet. On aurait peut-être pu, pour quelqu'un qui veut mettre une pompe, peut-être s'étendre un peu plus sur le sujet. » 


\subsection{Quel est le chapitre ou le sous-chapitre qui a le moins intéressé les patients ?}

Cette partie correspond à l'analyse des réponses obtenues à la question : "Quel est le chapitre ou le sous-chapitre qui vous a semblé le moins intéressant ou qui répond le moins à des événements de votre vie quotidienne? »

Trois patients ont répondu que le chapitre le moins intéressant pour eux ou répondant le moins à des événements de leur vie quotidienne était le chapitre 1: « Mes objectifs glycémiques ». Il s’agissait des patients $n^{\circ} 3,7$ et 8 .

Le patient $\mathrm{n}^{\circ} 3$ a précisé : "parce que mes objectifs glycémiques avaient déjà été bien définis. Oui, on en avait déjà bien discuté ici pendant ma semaine d'hospitalisation. Donc du coup c'est vrai que c'est pas mal d'avoir un rappel, mais c'est vrai que ce n'est pas non plus celui qui me semblait le plus nécessaire. »

Le patient $n^{\circ} 8$ a dit : «peut-être ce qui est plus général. Peut-être dans le 1, le début du 1, tout ce qui reprend vraiment les taux en-dessous desquels il ne faut pas être, les taux audessus desquels il ne faut pas être etc. Ça en général, vu que c'est quelque chose quand même d'important qu'on utilise tous les jours on le sait. On va dire vraiment les bases, ce qui nous a déjà été dit à l'hôpital c'est peut-être ce qui serait le moins utile dans le chapitre 1 mais après le reste franchement tout est bien enfin c'est bien de le rappeler quoi. »

Six patients ont répondu que le chapitre le moins intéressant pour eux ou répondant le moins à des événements de leur vie quotidienne était le chapitre 2 : « $\underline{\text { Mes hypos, mes }}$ hypers $»$. Il s'agissait des patients $n^{\circ} 2,4,5,6,9$ et 11 .

Les patients $2,4,5$ et 6 ont précisé qu'il s'agissait du chapitre 2.3 : « J'ai des problèmes d'hypos sévères ».

La patiente ${ }^{\circ} 9$ a précisé qu'il s'agissait du chapitre 2.1 : « Comment savoir où j’en suis au niveau de ma glycémie ? » qu'elle trouvait redondant avec le chapitre 1 : «je sais que je n'ai pas regardé le début du chapitre 2 parce que ça reprenait... comme j'avais regardé la vidéo la veille du ler chapitre, j'avais passé un peu directement le début du $2^{\text {ème }}$ chapitre parce que ça reprenait vraiment le 1 ... ». La patiente $\mathrm{n}^{\circ} 11$ a précisé : «parce que c'est quelque chose que je sais déjà. Ce n'est pas que je gère mais... enfin quand je vais avoir une hypo je le sais enfin je le sens, je le sais. » 
Aucun des patients n'a répondu que le chapitre le moins intéressant pour lui ou répondant le moins à des événements de sa vie quotidienne était le chapitre 3 : « $\underline{\text { Mes insulines } ~}$.

Deux patients ont répondu qu'aucun des chapitres était moins intéressant ou répondait le moins à des événements de leur vie quotidienne. Il s'agissait des patients $n^{\circ} 1$ et 10 .

Le patient $\mathrm{n}^{\circ} 10$ a précisé : «ils étaient tous intéressants et puis bon on est concerné par tous les chapitres dans notre vie quotidienne malgré tout. Non, moi j'ai trouvé qu'ils étaient tous intéressants. »

\subsection{Quel est le chapitre ou le sous-chapitre qui a le plus intéressé les patients?}

Cette partie correspond à l'analyse des réponses obtenues à la question : "Quel est le chapitre ou le sous-chapitre qui vous a semblé le plus intéressant ou le plus utile pour gérer votre diabète au quotidien ? »

Deux patients ont répondu que le chapitre le plus intéressant ou le plus utile pour gérer leur diabète au quotidien était le chapitre 1 : « Mes objectifs glycémiques ». Il s'agissait des patients $\mathrm{n}^{\circ} 2$ et 9 .

Le patient $\mathrm{n}^{\circ} 2$ a précisé : «moi j’ai bien aimé le $1^{\text {er }}$ chapitre avec l'enchainement des témoignages qui sont bien structurés, qui passent étape par étape et puis confronter les deux points de vue entre un jeune en quelque sorte et une personne un peu plus adulte. »

La patiente $\mathrm{n}^{\circ} 9$ a dit : «ce qui m'a le plus intéressée c'était dans le chapitre 1. Il y a un témoignage de la dame, quand elle dit que son objectif à elle c'est d'être dans les 1.40 par exemple pour ne pas être un poids pour ses coéquipiers ou ses collègues. Je pense que c'est ça qui m'a le plus interpellée. C'était vraiment je pense quelque chose qui me pesais de vouloir trop être basse. C'est pour ça que je faisais hypos sur hypos parce que dans ma tête ce n'était pas comme ça qu'il fallait... Avant je faisais en moyenne, en moyenne parce que ça m'arrivait d'en faire plus ou moins, mais c'était deux hypos par jour. Là ça fait une semaine que j'en ai pas fait. »

Six patients ont répondu que le chapitre le plus intéressant ou le plus utile pour gérer leur diabète au quotidien était le chapitre 2 : «Mes hypos, mes hypers ». Il s'agissait des patients $\mathrm{n}^{\circ} 1,3,5,6,7$ et 10 .

Les patients $\mathrm{n}^{\circ} 1$ et 7 ont plus particulièrement apprécié le chapitre 2.2 : « Comment je traite mes hypos et mes hypers ? » 
Les patients $\mathrm{n}^{\circ} 3,5$ et 6 ont plus particulièrement apprécié le chapitre $2.4:$ « Je veux aller plus loin dans l'analyse de l'estimation de ma glycémie ».

La patiente $\mathrm{n}^{\circ} 10$ a aimé le chapitre 2 dans son ensemble, elle a précisé : «même dans la forme je l'ai trouvé plus vivant. Enfin moi le 2 c'est vrai que je l'ai bien aimé. Au quotidien c'est ce qui me servira le plus oui. »

Deux patients ont répondu que le chapitre le plus intéressant ou le plus utile pour gérer leur diabète au quotidien était le chapitre 3 : « Mes insulines ». Il s'agissait des patients $\mathrm{n}^{\circ} 8$ et 11 .

Le patient $\mathrm{n}^{\circ} 8$ a précisé : «c'est le plus pratique et puis c'est vrai que tout ce qui est hypos et hypers c'est vraiment $d u$ ressenti, bon à part les infos que je vous ai précisées qui étaient intéressantes, mais après c'est vraiment quelque chose qu'on vit seul donc à la limite on consulte mais après on met en application seul. Mais «Mes insulines » je pense que ça peut éviter pas mal de mails ou de questions pas forcément utiles dans un premier temps à notre diabéto. »

Le patient $n^{\circ} \mathbf{4}$ a répondu que tous les chapitres étaient intéressants ou utiles pour gérer son diabète au quotidien.

\subsection{Impact de la participation à une action éducative antérieure et de l'ancienneté du diagnostic sur l'intérêt perçu vis-à-vis des différents chapitres}

\begin{tabular}{|c|c|c|c|c|c|c|}
\cline { 2 - 6 } \multicolumn{1}{c|}{} & \multicolumn{2}{c|}{ Le moins intéressant } & \multicolumn{2}{c|}{ Le plus intéressant } \\
\cline { 2 - 6 } & ETP & Pas ETP & Total & ETP & Pas ETP & Total \\
\hline $\begin{array}{c}\text { Chapitre 1 : Mes objectifs } \\
\text { glycémiques }\end{array}$ & 1 & 2 & 3 & 1 & 1 & 2 \\
\hline $\begin{array}{c}\text { Chapitre 2: Mes hypos, mes hypers } \\
2.1: \text { « Comment savoir où j'en suis au } \\
\text { niveau de ma glycémie ?» }\end{array}$ & 1 & 1 & $\mathbf{2}$ & 0 & 0 & $\mathbf{0}$ \\
\hline $\begin{array}{c}2.2: \text { « Comment je traite mes hypers } \\
\text { et mes hypos ?» }\end{array}$ & 0 & 0 & $\mathbf{0}$ & 1 & 1 & $\mathbf{2}$ \\
\hline $\begin{array}{c}2.3: \text { «'ai des problèmes d'hypos } \\
\text { sévères » }\end{array}$ & 2 & 2 & $\mathbf{4}$ & 0 & 0 & $\mathbf{0}$ \\
\hline $\begin{array}{c}2.4: \text { «Je veux aller plus loin dans } \\
\text { l'analyse de l'estimation de ma } \\
\text { glycémie » }\end{array}$ & 0 & 0 & $\mathbf{0}$ & 1 & 2 & $\mathbf{3}$ \\
\hline Dans son ensemble & 0 & 0 & $\mathbf{0}$ & 1 & 0 & $\mathbf{1}$ \\
\hline Chapitre 3 : Mes insulines & 0 & 0 & $\mathbf{0}$ & 1 & 1 & 2 \\
\hline
\end{tabular}

Tableau 3 : Impact de la participation à une action éducative antérieure sur l'intérêt perçu vis-à-vis des différents chapitres 
De plus, deux patients ont déclaré qu'aucun des chapitres ou sous-chapitres n'était moins intéressant ou répondait moins à des événements de leur vie quotidienne. L'un avait bénéficié d'une ETP antérieure, l'autre non.

Et un patient a jugé les trois chapitres comme les plus intéressants ou répondant le plus à des événements de sa vie quotidienne. Il n'avait pas bénéficié d'une ETP antérieure.

On constate que le chapitre 1 «Mes objectifs glycémiques » a été désigné comme le plus intéressant ou le plus utile par deux patients. L'ancienneté moyenne de leur diabète était de 8 mois. L'un avait bénéficié d'une ETP antérieure, l'autre non. Il a été désigné comme le moins intéressant ou le moins utile au quotidien par trois patients. L'ancienneté moyenne de leur diabète était de 6 mois et $1 \frac{1}{2}$. L'un avait bénéficié d'une ETP antérieure, les deux autres non.

Dans cet échantillon, l'ancienneté du diabète ou la délivrance d'une ETP antérieure ou non ne semblent pas déterminer le fait de trouver un chapitre sur les objectifs glycémiques intéressant ou utile ou non. Selon les réponses apportées par les patients interrogés cela pourrait être déterminé par l'anxiété vis-à-vis de la maladie, la volonté de trop bien faire, la culpabilisation de ne pas faire assez bien ou la détermination des objectifs fixés initialement.

Le chapitre 2 a été cité par six patients comme le plus intéressant ou le plus utile. Il a été cité également par six patients comme étant le moins intéressant ou répondant le moins à des événements de leur quotidien. Si l'on s'intéresse à ses sous-chapitres, on observe que les sous-chapitres 2.2 «Comment je traite mes hypers et mes hypos? » et 2.4 « Je veux aller plus loin dans l'analyse de l'estimation de ma glycémie » ont semblé les plus intéressants ou les plus utiles et que les sous-chapitres 2.1 : «Comment savoir où j’en suis au niveau de ma glycémie ? » et 2.3 : « J'ai des problèmes d'hypos sévères » ont semblé les moins intéressants ou répondant le moins à des événements de leur quotidien pour les patients de cet échantillon. Il n'y a pas de différence dans les réponses en fonction de l'ancienneté du diabète et de l'existence ou non d'une ETP antérieure.

En ce qui concerne le chapitre 3 «Mes insulines », aucun des patients ne l'a désigné comme le moins intéressant ou répondant le moins à des événements de leur vie quotidienne. Deux patients l'ont cité comme le plus intéressant ou le plus utile. L'un avait un diabète diagnostiqué depuis 5 mois et n'avait pas bénéficié d'ETP et l'autre avait un diabète diagnostiqué depuis 35 mois et avait bénéficié d'une ETP antérieure. 


\subsection{Quels sont les thèmes futurs envisagés par les patients ?}

Tous les patients se sont dit intéressés par les deux chapitres en cours de réalisation à savoir le chapitre 4 «Mon alimentation » et le chapitre 5 «Mes activités physiques ». Tous ont souhaités être informés lorsque ceux-ci seraient disponibles.

Le patient $\mathrm{n}^{\circ} 2$ a précisé qu'il était surtout en attente des chapitres concernant l'alcool et le sport; le patient $\mathrm{n}^{\circ} 3$ l'insulinothérapie fonctionnelle ; la patiente $\mathrm{n}^{\circ} 5$ les activités sportives et la patiente $\mathrm{n}^{\circ} 7$ l'alimentation et notamment apprendre à compter les glucides et à prendre en compte l'alcool.

En réponse à la question : «Est-ce qu'il y a un thème qui n'est pas abordé dans le site INDIGO et que vous auriez aimé trouver ou que vous aimeriez trouver dans le futur?», nous avons obtenu les réponses suivantes :

Le patient $n^{\circ} 3$ a proposé un chapitre sur l'histoire naturelle et l'évolution du diabète de type 1 .

Le patient $\mathrm{n}^{\circ} 8$ a envisagé un chapitre qui aborderait les principaux chiffres du diabète (nombre de patients diabétiques de type 1, de type 2, âge moyen d'apparition...) et ses causes d'apparition.

La patiente $\mathrm{n}^{\circ} 7$ a proposé une introduction basée sur des témoignages : «une introduction plus... psychologique sur la façon dont on ressent et on vit cette maladie chronique. Parce que quand on a fait un tour de table à la formation de diabétiques de type 1, la première question qu'on nous a posé c'est : "Qu'est-ce que vous avez ressenti, ça vous fait quoi d'être diabétique de type 1 »? Et on a tous parlé et j'ai trouvé ça très intéressant d'entendre les témoignages d'autres diabétiques comme moi. Et en plus, on voit qu'il y a des points communs, des différences, enfin, on l'a vécu de façon plus ou moins différente et peut-être que ça manque un peu. » Elle a précisé : "Mais en même temps, il ne faut pas que ce soit trop anxiogène pour celui qui regarde donc est-ce qu'il faut forcément parler du vécu de la maladie d'une façon générale en fait ?»

Le patient $\mathrm{n}^{\circ} 8$ a proposé un chapitre sur les différents modes de traitement : «il y a déjà les différents types d'insuline mais peut-être sur les différents modes de traitement du style injections, pompe. Peut-être une présentation des pour et des contre de la pompe, de l'injection. Je sais que c'est abordé dans les insulines mais peut-être un chapitre à part entière carrément sur les modes de traitement existants. Enfin donner un peu des infos qui 
peut-être dégrossiraient l'information avant de poser des questions à notre diabéto en consultation. »

La patiente $\mathrm{n}^{\circ} 10$ a également proposé un chapitre à part entière sur la pompe à insuline : «on nous en a parlé pendant l'hospitalisation mais on ne sait pas trop comment ça fonctionne. Pour savoir si ça nous conviendrait, pour peut-être mieux connaître aussi. » La patiente $\mathrm{n}^{\circ} 11$ avait regretté le manque d'informations sur la pompe à insuline dans le chapitre « Mes insulines ».

Les patients $n^{\circ} 8$ et 11 ont souhaité trouver un chapitre sur la recherche et les avancées dans le domaine du diabète de type 1: "peut-être un point sur la recherche éventuellement, les avancées, ça peut être bien. Vu que c'est un secteur qui est... enfin je sais que pour le diabète c'est assez dynamique, c'est peut-être pas mal de tenir au courant de l'évolution » (8).

La patiente $n^{\circ} 9$ a soumis l'idée d'un chapitre sur les voyages et les excursions : «comment gérer son diabète quand on n'est pas dans le schéma habituel de vie, quand on n'est pas tous les soirs chez soi."

La patiente $n^{\circ} 6$ avait déclaré avoir des interrogations à ce sujet également.

Les cinq autres patients n'ont pas fait de proposition. Ils considéraient que le site serait complet avec les deux chapitres en cours de réalisation «Mon alimentation » et «Mes activités physiques ».

\subsection{Quelle est la note de satisfaction globale attribuée par les patients?}

Cette partie correspond à l'analyse des réponses obtenues à la question : « Sur une échelle de 0 à 10, quelle est votre satisfaction globale vis-à-vis d'INDIGO ? »

\begin{tabular}{|c|c|c|c|c|c|c|c|c|c|c|c|}
\hline Patient & $\mathbf{1}$ & $\mathbf{2}$ & $\mathbf{3}$ & $\mathbf{4}$ & $\mathbf{5}$ & $\mathbf{6}$ & $\mathbf{7}$ & $\mathbf{8}$ & $\mathbf{9}$ & $\mathbf{1 0}$ & $\mathbf{1 1}$ \\
\hline Note & 10 & 8 & 7 & 9 & 10 & 9 & 7 & 8 & 10 & 10 & 9 \\
\hline
\end{tabular}

Tableau 4 : Notes de satisfaction globale attribuées par les patients de l'échantillon

Les patients ont attribué la note moyenne de $\mathbf{8 , 8 / 1 0}$ à leur satisfaction globale vis-à-vis du site INDIGO. 


\section{Modalités d'utilisation du site INDIGO par les patients interrogés}

Afin de conseiller les futurs utilisateurs sur les différentes façons possibles d'utiliser le site INDIGO, nous avons étudié la manière dont chaque patient de l'échantillon a procédé.

\subsection{En entier ou partiellement ?}

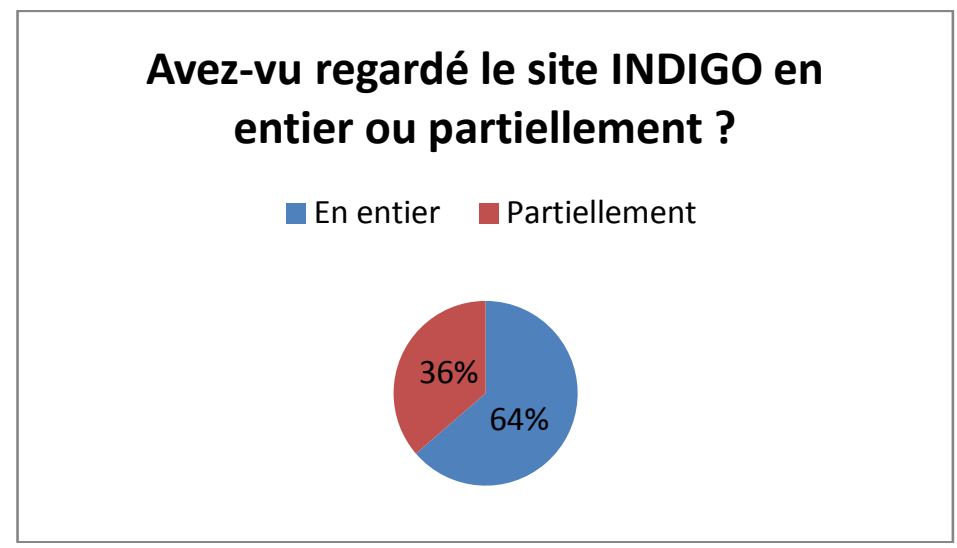

Figure 33 : En entier ou partiellement?

7 patients $(64 \%)$ ont regardé le site INDIGO dans sa totalité.

Le patient $\mathrm{n}^{\circ} 2$ a regardé rapidement le sous-chapitre 2.1 : «il y a juste peut-être un chapitre que j'ai un peu passé, c'est le chapitre "Comment savoir où j'en suis au niveau de ma glycémie?". Il me semblait que ça faisait un peu redondant avec le chapitre précédent. »

Le patient $n^{\circ} 3$ n'a pas regardé le sous-chapitre 2.3 « J'ai des problèmes d'hypos sévères » parce qu'il ne se sentait pas concerné. Il n'a pas vu non plus le chapitre 3 « Mes inulines ».

La patiente $n^{\circ} 7$ n'a pas vu le chapitre 1 «Mes objectifs glycémiques » en entier car elle l'a trouvé troublant: "j'avoue que je l'ai regardé assez rapidement pour éviter de me retrouver face à une situation où je vais me dire "Bon ben ça va pas très bien en fait, je ne suis pas assez rigoureuse. » »

La patiente $n^{\circ} 9$ n'a pas vu le chapitre 3 « Mes insulines» en raison de problèmes techniques puis par manque de temps : «la première fois c'était plutôt parce que ça ramait trop. J'avais une mauvaise connexion. Depuis j'ai changé de serveur. Mais après c'était plutôt par manque de temps. Avec mes horaires je ne me suis pas repenchée dessus. Et puis 
là avant de vous rappeler j'ai voulu, mais du coup j'ai re-regardé que le $1^{\text {er }}$ et les autres vu qu'on demandait le code et que je ne m'en souvenais pas... »

Elle n'a pas vu le chapitre 2 «Mes hypos, mes hypers» en entier en raison de redondances avec le chapitre 1 et au vu de ses connaissances antérieures. Elle l'a également trouvé moins attractif : "il y a des parties que j'ai passées. J'avoue que j'ai moins accroché. Ça répétait certaines choses $d u 1^{e r}$, ça m'a moins apporté de choses. Il y a des choses que je savais aussi. Et je l'ai trouvé moins léger que le $1^{\text {er }}$, c'est ce qui m'a poussée à avancer plus vite dans le chapitre. »

\subsection{En une ou plusieurs fois ?}

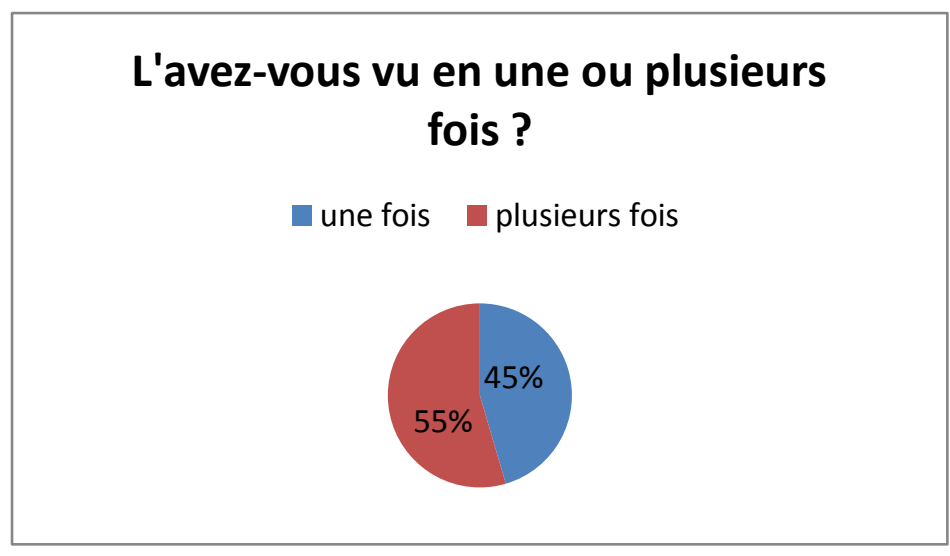

Figure 34 : En une ou en plusieurs fois ?

5 patients $(45 \%)$ ont vu le site INDIGO en une seule fois. L'un d'eux était le patient $\mathrm{n}^{\circ} 3$ qui n'a vu que les deux premiers chapitres.

La patiente $\mathrm{n}^{\circ} 1 \mathrm{n}$ 'a pas trouvé le visionnage fastidieux : «au début, je me suis dit " J'ai mon rendez-vous dans pas longtemps, il faudrait que je pense à regarder. "J'ai regardé le premier et puis au final je les ai tous faits dans la foulée. J'ai commencé par le chapitre 1 et les parties se sont enchainées les unes aux autres et puis je me suis dit autant passer au chapitre 2 et au chapitre 3. Je sais que j'y ai passé du temps mais je ne me suis pas ennuyée devant. » Mais elle s'est déconcentrée : “ j'y suis revenue pour voir la $4^{\text {ème }}$ partie du chapitre 3. Parce que comme je les ai faits tous d'affilée, le dernier j'étais un peu plus fatiguée et du coup je voulais être sûre de ne pas être passée à côté de quelque chose d'important. Le deuxième visionnage était un peu indispensable parce que je pense que j'étais un petit peu endormie à ce moment-là. » 
Le patient $\mathrm{n}^{\circ} 3$ a jugé trop long le fait de voir l'ensemble des chapitres en une fois : "le chapitre 3 je ne l'ai pas vu parce que disons que quand je m'y suis mis ça a duré à peu près... Parce que j'ai vraiment tout vu quoi, toutes les vidéos complètement, j'ai mis 1 heure et demie. Donc après je me suis arrêté et puis c'est vrai que je ne m'y suis pas replongé. »

Le patient $\mathrm{n}^{\circ} 8$ a précisé qu'il y avait des répétitions si l'on visionnait l'ensemble en une fois : "ça faisait peut-être un petit peu long les trois en une fois. Deux c'est bien, trois c'est un peu long. En fait c'est que, surtout entre le 1 et le 2, il y a pas mal... on peut vraiment les prendre à part parce que j'ai l'impression qu'il y a certaines infos qui se recoupent entre chaque et du coup c'est vrai que si on s'enchaine les trois à la suite ça peut faire un peu répétition. »

Deux patientes ont précisé que l'impression de «longueur» était due à la quantité d'informations : "c'est vrai que tout regarder d'un coup, c'était long. Ça fait beaucoup d'informations » (10); "je ne sais pas si c'est parce qu'aujourd'hui je travaillais et que j'étais peut-être plus fatiguée, mais je l'ai trouvé très long en fait. Si on parcourt tout le site j'ai trouvé ça assez long. Peut-être parce qu'en fait c'est très complet, ce qui est très bien. Mais du coup, voilà, je crois que c'était assez long. Mais ce n'était pas de l'ennui » (11).

Les six autres patients l'ont regardé en plusieurs fois.

Le patient $\mathrm{n}^{\circ} 2$ a déclaré : "au bout d'un moment ça fait beaucoup d'informations quand même, enfin vu qu'il y a quand même quatre heures de vidéos je crois, enfin quelque chose comme ça. Du coup c'est pas mal de faire une petite pause et de le voir étape par étape. »

La patiente $\mathrm{n}^{\circ} 5$ a dit : "c'était à la fin de la journée mais après c'est vrai que c'est un peu lourd de faire les trois chapitres d'un coup. Petit à petit je suivais de moins en moins donc j'ai préféré le faire en deux fois. »

Au total, parmi les 5 patients qui ont vu le site INDIGO en une fois, 4 ont trouvé le visionnage long. Les autres ont découpé le visionnage car cela leur a semblé fastidieux, en particulier en raison de la quantité importante d'informations. 


\subsection{Dans l'ordre proposé des chapitres ou non?}

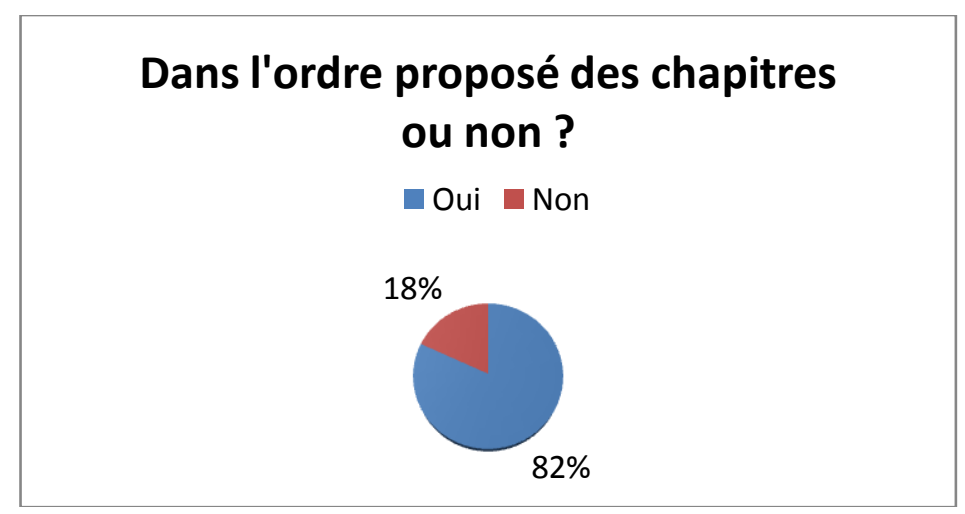

Figure 35 : Dans l'ordre proposé des chapitres ou non ?

9 patients $(82 \%)$ ont regardé les chapitres dans l'ordre proposé : « Mes objectifs glycémiques » puis « Mes hypos, mes hypers » puis « Mes insulines ».

Sur ces 9 patients, 7 patients (63,6\% de l'ensemble de l'échantillon) ont également regardé les sous-chapitres dans l'ordre proposé.

Les deux autres patients ont regardé dans chaque chapitre les sous-chapitres par ordre d'intérêt croissant. La patiente $n^{\circ} 6$ a choisi de commencer par le chapitre 3 « Mes insulines » : «je l'ai regardé étape par étape. Je ne regarde que ce qui m'intéresse, sur ce que je vis en ce moment. Au tout début c'était le chapitre " Mes insulines ». » La patiente $\mathrm{n}^{\circ} 9$ a quant à elle commencé par le chapitre 2 «Mes hypos, mes hypers »: «parce que c'est tellement la base de tout que je me suis dit «Tiens je vais voir exactement ce qu'ils disent ». »

\subsection{Seul ou accompagné ?}

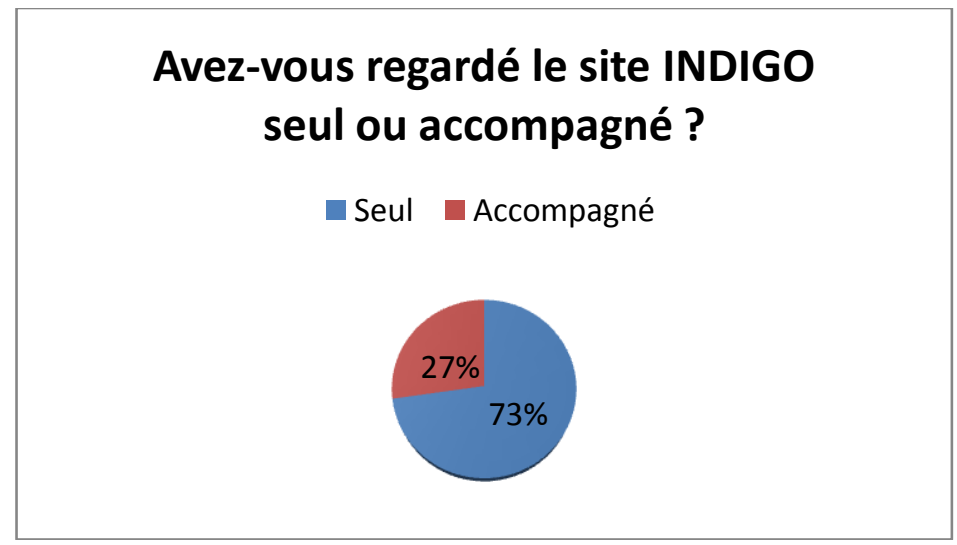

Figure 36 : Seul ou accompagné ? 
8 patients $(73 \%)$ ont regardé le site INDIGO seuls. 3 patients $(27 \%)$ l'ont, en partie, regardé accompagnés : le patient $\mathrm{n}^{\circ} 2$ avec sa conjointe, la patiente $\mathrm{n}^{\circ} 5$ avec son frère et la patiente $\mathrm{n}^{\circ} 7$ avec son conjoint. Aucun patient ne l'a visionné accompagné dans sa totalité.

\subsection{Les patients pensent-ils retourner sur le site INDIGO ?}

Les deux patients qui n'ont pas vu le chapitre 3 « Mes insulines » ont l'intention de le voir prochainement. 8 patients ont déclaré qu'ils souhaitaient retourner sur le site ultérieurement pour revisionner tout ou partie des trois premiers chapitres. Il s'agissait des patients $\mathrm{n}^{\circ} 1,2,3,6,7,8,9,10$. Le sujet n'a pas été abordé par les patients $\mathrm{n}^{\circ} 4$ et 5 . La patiente $n^{\circ} 11$ ne souhaitait pas revoir les chapitres déjà vus.

Tous souhaitaient voir les chapitres 4 et 5 .

\subsection{A quel moment proposer le site INDIGO ?}

Cette question n'a pas été posée de manière systématique aux patients car elle ne faisait pas partie du guide d'entretien. Toutefois le thème a été abordé par certains patients pendant l'entretien semi-dirigé.

\subsubsection{En fonction de l'ancienneté du diabète}

Une patiente a estimé que le meilleur moment était celui du diagnostic : «je pense qu'il faudrait le proposer aux personnes qui viennent juste d'être diagnostiquées, qui viennent juste de voir qu'ils sont diabétiques. Je trouve que c'est très bien parce que ça explique en fait tout... tout le diabète on va dire »(11, diagnostiquée depuis 35 mois).

Deux patients ont estimé qu'il ne fallait pas le voir « trop tôt » : «la première fois je l'ai regardé juste après que je sois sorti de l'hôpital ou presque, donc là je ne comprenais pas encore tout; je n'étais peut-être pas prêt comme état d'esprit et les infos j'en avais déjà. Je pouvais encore peut-être l'élaborer un peu en moi» (4, diagnostiqué depuis 3 mois) ; "ça ne sert à rien de le consulter tout de suite après la sortie d'hospitalisation. Il faut vraiment un peu de temps avant de comprendre tout ce qui est expliqué. Il faut avoir un peu vécu ce que sont les hypers et les hypos pour savoir de quoi parle le site, comprendre les témoignages » (8, diagnostiqué depuis 5 mois).

Une autre patiente a estimé qu'il aurait fallu idéalement qu'elle le visionne au moment du diagnostic ou à distance, sa première année après le diagnostic ayant été centrée sur le 
diabète: «c'est ma première année de diabète, je me suis renseignée, j'ai été bien entourée, encadrée par le personnel médical. Pour l'instant je suis plutôt au courant de toutes ces choses-là. La première année ça a été le diabète, le diabète, donc ce n'est pas forcément en ce moment où j'ai le plus envie de passer du temps sur le diabète. En revanche sur du long terme quand peut-être on a un suivi juste avec son diabétologue ça permet de remettre des choses à jour parce qu'on commence à prendre des réflexes, on se pose moins de questions et on peut partir sur des directions moins rigoureuses. Je pense qu'il faudrait le proposer en sortie de première hospitalisation parce que quand on découvre le diabète tout nous parait invraisemblable, rien n'est naturel. Dans les deux premiers mois je pense que de pouvoir me référer à un site comme ça officiel, ça m'aurait rassurée. Si je l'avais eu en sortant de l'hôpital ça aurait été très utile pour moi, ça m'aurait rassurée » (7, diagnostiquée depuis 11 mois).

Une autre patiente a estimé que le site INDIGO avait un intérêt au début de la maladie comme à distance: "ça nous interpelle beaucoup, peut-être parce que ça fait pas longtemps qu'on a le diabète. Je me demandais si quelqu'un qui avait depuis 20 ans du diabète regarderait le site. Mais il y a des informations ça serait dommage qu'il passe à côté en se disant "ça fait 20 ans que je l'ai, je m'en fous un peu de savoir, je sais comment le gérer »»(10, diagnostiquée depuis 9 mois).

\subsubsection{En fonction des événements extérieurs}

Une patiente a déclaré : «j'avais des mauvais résultats de diabète et ça me stressait, je n'étais pas dans l'état d'esprit. Je pense que je serais plus capable de le regarder aujourd'hui, j'étais peut-être un peu trop stressée la dernière fois. Il ne faut déjà pas le regarder le soir avec la fatigue et tout. Et puis j'étais en train de chercher du travail, je n'en trouvais pas, ce n'était pas le moment» (9, diagnostiquée depuis 7 mois).

\subsection{Les enfants et adolescents diabétiques et leurs parents}

Une patiente a envisagé d'étendre le site INDIGO à un public d'enfants ou d'adolescents et à leurs parents : «il n'y a pas de jeunes jeunes dans les témoignages, de 12-13-14-15 ans, donc peut-être que c'est dommage, je ne sais pas. Je pense notamment aux parents d'enfants diabétiques. Je pense que c'est quelque chose qui pourrait énormément rassurer les parents. Est-ce que l'enfant de 10-12 ans irait regarder ça peut-être pas mais les parents qui ont vraiment besoin d'un support... »(7). 


\section{Patiente $\mathrm{n}^{\circ} 12$}

Le choix a été fait d'interroger de plus une patiente dont le diabète a été diagnostiqué il y a 27 ans afin d'identifier l'impact potentiel de la durée de vécu avec la maladie sur l'utilisation et la satisfaction de l'outil.

\subsection{L'entretien}

A la convenance de la patiente, l'entretien s'est déroulé par téléphone. Il a duré 33 minutes.

\subsection{Caractéristiques socioprofessionnelles, médicales et utilisation d'Internet}

\begin{tabular}{|c|c|c|c|c|c|c|c|}
\hline & Sexe & Age & $\begin{array}{c}\text { Niveau } \\
\text { d'études }\end{array}$ & Profession & $\begin{array}{c}\text { Ancienneté } \\
\text { du diabète }\end{array}$ & Stylo/Pompe & $\begin{array}{c}\text { Participation ET } \\
\text { «traditionnelle » }\end{array}$ \\
\hline $\begin{array}{c}\text { Patiente } \\
\mathbf{n}^{\circ} \mathbf{1 2}\end{array}$ & Femme & $\mathbf{3 5}$ ans & $\begin{array}{c}\text { Bac +3 et } \\
\text { plus }\end{array}$ & $\begin{array}{c}\text { Professeur des } \\
\text { écoles }\end{array}$ & $\mathbf{2 7}$ ans & Pompe & Oui \\
\hline
\end{tabular}

Tableau 5 : Caractéristiques socioprofessionnelles et médicales de la patiente $\mathrm{n}^{\circ} 12$

La patiente $\mathrm{n}^{\circ} 12$ a déclaré qu'elle possédait une connexion Internet à son domicile mais pas sur son lieu de travail. Elle se définissait comme une utilisatrice inconditionnelle d'Internet et/ou de multimédia. Elle avait déjà effectué des recherches concernant sa santé et notamment son diabète sur Internet, sans préciser sur quels sites.

\subsection{Analyse du questionnaire dirigé et des entretiens semi- dirigés}

\subsubsection{Appréciations relatives à la qualité du contenu}

Les réponses de cette patiente au questionnaire dirigé ne se sont pas démarquées des réponses des autres patients de l'échantillon concernant la qualité du contenu.

L'entretien semi-dirigé ne nous a pas apporté d'éléments supplémentaires.

\subsubsection{Appréciations relatives à la qualité pédagogique}

Les réponses de la patiente $\mathrm{n}^{\circ} 12$ au questionnaire dirigé ne se sont pas démarquées des réponses des autres patients de l'échantillon concernant la qualité pédagogique.

Lors de l'entretien semi-dirigé elle a donné quelques précisions.

Elle a apprécié les schémas: «j'ai trouvé que c'était bien de montrer des graphiques, c'était assez parlant. » 
Elle a également aimé les témoignages. Tout comme les patients $\mathrm{n}^{\circ} 3$ et 11 , ceux-ci lui ont permis de se confronter à d'autres personnes diabétiques de type 1: "c'est sympa de voir d'autres gens qui peuvent être confrontés aux mêmes choses que ce que l'on vit tous les jours. C'est inhabituel pour moi parce que je connais très peu d'autres personnes qui ont la même chose que moi. »

Elle a également pu s'identifier aux témoins tout comme les patients $n^{\circ} 3,5,7,8$ et 11 : «avec les films on peut s'identifier aux personnes qui parlent. »

Enfin comme le patient $n^{\circ} 2$ elle s'est demandée si les témoins étaient des patients diabétiques ou des acteurs : «je ne sais pas si c'est des gens vraiment diabétiques ou des acteurs. »

Comme tous les patients de l'échantillon, elle conseillerait le site INDIGO à un patient diabétique de type 1.

Elle le conseillerait également à un membre de son entourage. Mais elle a précisé : «mais mes proches connaissent bien mon problème, ils n'ont pas forcément de questions par rapport à ça.»

\subsubsection{Appréciations relatives à la qualité du support}

Dans le questionnaire dirigé, à la question $n^{\circ} 25$ : «Les voix off sont-elles agréables ? », la patiente $\mathrm{n}^{\circ} 12$ a attribué la note de $\mathbf{0 , 5 / 1 0}$. Les patientes $\mathrm{n}^{\circ} 10$ et 11 avaient attribué à cette question la note de 5/10 et la note moyenne attribuée par l'échantillon était de 8/10. Elle n'a pas fait de commentaires à ce sujet lors de l'entretien semi-dirigé.

A la question $n^{\circ} 33$ : « La navigation est-elle aisée ? », elle a attribué la note de $\mathbf{0 , 5 / 1 0}$. La notre minimale attribuée par l'échantillon à cette question était de 5/10 et la moyenne était de $8,3 / 10$.

Lors de l'entretien semi-dirigé elle a déclaré avoir été surprise par la forme du site INDIGO : «j'ai déjà fait d'autres recherches sur Internet, la présentation n'est pas forcément comme ça. J'ai eu l'impression que c'était plus un DVD ou un diaporama que vraiment un site Internet. Il y a des vidéos, des graphiques, des textes. Pour trouver une information il fallait écouter, enfin passer toutes les diapositives. »

Pour elle cette forme a été contraignante : «la forme j'ai trouvé que c'était un petit peu long. C'est vrai que je préfère lire des choses pour aller assez vite et là on a à la fois une voix qui parle, un message écrit, des vidéos. J'ai trouvé que c'était un peu lourd. En fait j'ai 
dû passer beaucoup de temps à passer d'une diapo à l'autre et j'aurais aimé pouvoir avoir autant d'informations mais en y consacrant un peu moins de temps. »

Elle a suggéré des améliorations à apporter au site : «pour certaines personnes ça peutêtre bien que ça soit sous cette forme mais peut-être avoir le choix entre avoir toutes les informations ou seulement une information texte par exemple et puis pouvoir cliquer si on veut approfondir par une vidéo ou quelque chose comme ça. J'aurais apprécié de pouvoir trouver les informations écrites de manière séparée pour pouvoir aller sur le site de manière plus rapide. »

Elle a également regretté l'absence de lien dans la navigation entre les chapitres : «ce qui m'a un peu gênée aussi c'est que quand on a fini un sous-chapitre on doit retourner au menu pour activer le sous-chapitre suivant, ça ne se fait pas de manière automatique si on veut voir tout le chapitre d'un coup. » Le patient $\mathrm{n}^{\circ} 2$ avait fait la même remarque.

Elle a suggéré : "j'aurais préféré que ça puisse s'enchainer aussi, que l'on puisse accéder au menu quand on veut, mais que l'on puisse aussi enchainer sans revenir par le menu. »

Elle a rencontré quelques difficultés techniques comme les patients $\mathrm{n}^{\circ} 9$ et 10 en rapport avec le temps de chargement : "j'ai un débit assez bon chez moi et les vidéos étaient quand même saccadées. Il y avait des temps d'attente, de chargement. »

\subsubsection{Appréciations relatives à la qualité de la conception}

Les réponses de cette patiente au questionnaire dirigé ne se sont pas démarquées des réponses des autres patients de l'échantillon concernant la qualité de la conception. L'entretien semi-dirigé ne nous a pas apporté d'éléments supplémentaires.

\subsubsection{Appréciation d'ensemble}

Dans le questionnaire dirigé, à la question $\mathrm{n}^{\circ} 38$ : « Le site INDIGO a-t-il répondu à vos besoins ? », la patiente $\mathrm{n}^{\circ} 12$ a attribué la note de 8/10. La moyenne attribuée à cette question par l'échantillon était de 8,6/10.

Lors de l'entretien semi-dirigé elle a déclaré : «j'ai pu apprendre et réviser des choses. » 


\subsection{Analyse des appréciations chapitre par chapitre à partir des entretiens semi-dirigés}

\subsubsection{Chapitre $1:$ « Mes objectifs glycémiques »}

La patiente $\mathrm{n}^{\circ} 12$ a déclaré avoir apprécié les témoignages dans ce chapitre, tout comme 6 des 11 patients de l'échantillon.

Elle n'a pas relevé d'élément négatif.

A la question: «Les informations ont-elles répondu aux questions que vous vous posiez sur vos objectifs glycémiques? » elle a répondu : «je les connais bien mais ça conforte ce que je pensais. »

\subsubsection{Chapitre 2 : «Mes hypos, mes hypers »}

Elle n'a pas relevé de point positif ni de point négatif dans ce chapitre.

\subsubsection{Chapitre 3 : «Mes insulines »}

La patiente $\mathrm{n}^{\circ} 12$ a aimé découvrir des informations plus précises sur l'insuline : la notion de pic d'action de l'insuline et la relation entre l'action de l'insuline et la température du corps : «l'histoire des pics d'action d'insuline, tout ça je n'en avais jamais entendu parler par exemple. Je ne savais pas non plus que quand on se pique sur une partie du corps qui est plus chaude ou si on prend un bain après avoir fait l'injection ça augmentait la vitesse d'action de l'insuline. Ça je ne savais pas du tout. Ça pourra me servir à mieux comprendre si j'ai une hypoglycémie que je n'arrive pas à expliquer. » La patiente $\mathrm{n}^{\circ} 10$ avait apprécié ces informations également.

Elle n'a pas relevé de point négatif concernant ce chapitre.

\subsubsection{Quel est le chapitre ou le sous-chapitre qui a le moins intéressé la patiente $n^{\circ} 12$ ?}

La patiente $\mathrm{n}^{\circ} 12$ a répondu le chapitre 2 «Mes hypos, mes hypers » : "parce que c'est quelque chose que je connais tellement bien. » 6 des 11 patients de l'échantillon avaient également répondu « le chapitre 2 » à cette question. 


\subsubsection{Quel est le chapitre ou le sous-chapitre qui a le plus intéressé la patiente $n^{\circ} 12$ ?}

La patiente $\mathrm{n}^{\circ} 12$ a répondu le chapitre 3 « Mes insulines » : «pour m'aider à gérer et par curiosité d'avoir plus de connaissances sur l'insuline. » 2 des 11 patients de l'échantillon avaient répondu « le chapitre 3 » à cette question.

\subsubsection{Quels sont les thèmes futurs envisagés par la patiente $n^{\circ} 12$ ?}

Comme tous les patients de l'échantillon, la patiente $\mathrm{n}^{\circ} 12$ a déclaré être intéressée par les deux chapitres en cours de réalisation et a souhaité être informée lorsque ceux-ci seraient disponibles. Elle a précisé : «dans le chapitre "Mon alimentation » moi ce que j’aimerais trouver c'est des informations sur l'insulinothérapie fonctionnelle. »

Elle a proposé un chapitre sur le diabète de type 1 et la grossesse : "je me suis posé beaucoup de questions quand j'étais enceinte de mes enfants, sur ce qu'il se passait quand on est enceinte, parce que ça perturbe beaucoup le diabète et là je pense qu'on est très en recherche de comprendre, de mieux connaître le diabète dans ce cas-là. J'ai fait beaucoup de recherches sur des forums, des choses comme ça à l'époque. Pour des futures mamans diabétiques je pense que ça pourrait être bien. »

\subsubsection{Quelle est la note de satisfaction globale attribuée par la patiente $\mathbf{n}^{\circ} 12$ ?}

Elle a attribué la note de 7/10. La moyenne de l'échantillon était de 8,8/10. Elle a déclaré : «moi j’ai bien aimé le contenu mais la forme m'a un petit peu dérangée. »

\subsection{Modalités d'utilisation du site INDIGO par la patiente $\mathrm{n}^{\circ} \mathbf{1 2}$}

Comme 36\% des patients de l'échantillon, elle n'a pas visionné le site INDIGO en entier. Elle a regardé plus rapidement plusieurs parties dans chaque chapitre parce que le titre lui laissait penser qu'elle maitrisait ces éléments et par manque de temps. Elle a malgré tout abordé tous les chapitres.

Comme 55\% des patients de l'échantillon, elle a visionné le site INDIGO en plusieurs fois. Elle l'a regardé dans l'ordre proposé comme $82 \%$ des patients de l'échantillon : « d'abord le premier chapitre, pour voir, par curiosité. Donc là j'ai suivi bien toutes les étapes, toutes les vidéos, les petits questionnaires, tout ça. Et après je suis allée me promener dans 
les chapitres suivants d'autres jours mais de manière ponctuelle. Je ne pense pas que j'ai tout vu mais je suis allée voir quelques séquences dans les chapitres. »

Comme $73 \%$ des patients de l'échantillon, elle l'a visionné seule.

Enfin elle a déclaré qu'elle retournerait ultérieurement voir les éléments qu'elle n'avait pas vus : «pour faire le tour, faire le point sur ce qui est montré, sur ce qui est dit. »

\subsection{A quel moment proposer le site INDIGO d'après la patiente $\mathbf{n}^{\circ} 12$ ?}

Elle a déclaré que le meilleur moment dans la vie d'un patient diabétique pour visionner le site INDIGO était selon elle dans les suites du diagnostic : «je pense que c'est plus adapté pour des gens qui sont nouvellement diabétiques et qui veulent comprendre leur maladie. » Mais elle a nuancé ses propos : «je pense que ça s'adresse à toute personne diabétique en quête d'informations supplémentaires. Il y a des moments où le diabète on en a jusque là, on n'a pas envie de s'en occuper et puis d'autres moments où on a envie de bien faire, on pense à son avenir, à sa santé et puis on veut essayer d'améliorer du mieux possible. »

Au total, la patiente $\mathrm{n}^{\circ} 12$ a déclaré que le site INDIGO correspondait à ses besoins en donnant une note de $\mathbf{8 / 1 0}$, note proche de la moyenne de l'échantillon qui était de $8,6 / 10$. Sa note de satisfaction globale était de 7/10 pour une moyenne de l'échantillon à 8,8/10. Elle a dit avoir appris et revu des choses mais a regretté la forme du site avec les vidéos et les informations délivrées par une voix off. Elle aurait préféré pouvoir faire le choix d'accéder à des informations écrites pour le consulter plus rapidement.

Elle a estimé que le site INDIGO devrait être proposé plutôt au début de la maladie mais elle est revenue sur ses propos en expliquant que dans la vie d'un patient diabétique il y a toujours des choses nouvelles à apprendre et des moments où l'on veut faire mieux pour sa santé. Selon elle, le site INDIGO pourrait donc être proposé à tout patient diabétique de type 1 en quête d'informations sur sa maladie. 
IV-Discussion 


\section{Discussion de la méthode}

\subsection{Les points forts}

\subsubsection{Les points forts de la méthode}

Le principal point fort de cette étude est l'utilisation du référentiel des critères de qualité d'un outil d'intervention en éducation pour la santé de l'INPES. ${ }^{28}$

D'après la loi de 2002 relative aux droits des malades et à la qualité du système de santé, l'INPES a notamment pour mission d'établir, en lien avec les professionnels concernés, les « critères de qualité pour les actions, les outils pédagogiques et les formations d'éducation thérapeutique et d'éducation pour la santé et de développer, valider et diffuser les référentiels de bonnes pratiques dans ces domaines $\gg \cdot{ }^{36} \mathrm{Ce}$ référentiel a été crée entre 2003 et 2005 dans le cadre de cette mission.

Son utilisation nous a permis de réaliser une analyse complète, reproductible et basée sur un référentiel reconnu, qui s'est appuyé pour son élaboration sur une méthode rigoureuse scientifiquement. En effet, ce référentiel a été élaboré par un groupe d'experts en évaluation et/ou en éducation pour la santé. Il est basé sur une analyse de la littérature et des expériences similaires existant au niveau national et international. Il a déjà servi à l'analyse de plus de 400 outils. L'INPES assure la diffusion de leur synthèse à l'ensemble des acteurs de l'éducation pour la santé à l'aide d'une base de données, la pédagothèque ${ }^{37}$, disponible sur son site Internet.

Cela rend également possible les comparaisons avec d'autres outils analysés selon les mêmes critères. A ce jour, ces critères n'ont pas été utilisés pour l'évaluation d'un site Internet d'éducation thérapeutique.

Un autre point fort de notre étude réside dans l'utilisation conjointe de deux méthodes d'analyse qualitative : l'entretien dirigé utilisé pour l'étude normative et l'entretien semidirigé qui a permis de réaliser une analyse approfondie des ressentis et des opinions des patients concernant le site Indigo et d'aborder les perspectives d'amélioration.

\subsubsection{Les points forts de l'échantillon}

Malgré la taille modeste de l'échantillon, la saturation des données a été obtenue, c'est-àdire que la collecte des données n'apportait plus d'informations suffisamment nouvelles pour justifier l'augmentation de la taille de l'échantillon. ${ }^{33}$ 


\subsection{Les points faibles}

\subsubsection{Les points faibles de la méthode}

\subsubsection{Biais de déclaration}

Les patients ont été présélectionnés en consultation durant la période d'inclusion de l'étude. Ils ont été informés de l'existence du site Indigo et de l'étude à venir par leur diabétologue et invités à consulter le site. Ceci a pu aboutir à un biais de déclaration car il est possible que les patients aient fait des commentaires plutôt positifs afin satisfaire leur diabétologue. Ceci d'autant plus que sept entretiens se sont déroulés dans le service de diabétologie du CHU de Grenoble, dont deux avant ou après un rendez-vous programmé avec leur diabétologue. Ceci a pu empêcher certains patients de s'exprimer librement.

\subsubsection{Biais de mémorisation}

Les patients ont été interrogés sur un site Internet dont le visionnage dure environ 3 heures et $1 / 2$. Ils peuvent avoir omis certains éléments ou avoir confondu les séquences et/ou les chapitres entre eux.

\subsubsection{Biais liés à l'enquêteur}

Le manque d'expérience de l'enquêtrice a pu amener à des erreurs dans la conduite des entretiens. Certaines questions ont pu être trop fermées, certains silences non respectés, certaines paroles interrompues ou encore certaines réponses influencées.

\subsubsection{Les points faibles de l'échantillon}

Aucun des patients n'a déclaré être un utilisateur occasionnel d'Internet et/ou de multimédia (100\% d'utilisateurs réguliers ou inconditionnels). Il aurait pourtant été intéressant d'obtenir l'avis de patients se définissant comme tels. 


\section{Discussion des résultats}

\subsection{Qualités et freins perçus - Perspectives d'amélioration}

\subsubsection{Le contenu}

Le premier point fort du site INDIGO semble être la qualité et la quantité des informations disponibles. Il sera complété par un chapitre sur l'alimentation et un chapitre sur l'activité physique. Tous les patients ont déclaré qu'ils souhaitaient voir ces chapitres. Les souhaits des patients à propos d'évolutions futures du contenu du site INDIGO concernent les différents modes de traitement du diabète de type 1 : pompe à insuline versus injections au stylo (27\%) ; les données épidémiologiques et physiopathologiques du diabète de type $1(18 \%)$; les avancées et les pistes de la recherche dans le domaine du diabète de type $1(18 \%)$; voyages, excursions et diabète de type $1(18 \%)$ et comment viton avec une maladie chronique $(18 \%)$.

Selon la patiente $\mathrm{n}^{\circ} 12$, diabétique depuis 27 ans et mère de deux enfants, il manque un chapitre sur le diabète de type 1 et la grossesse. Elle-même avait recherché des informations à ce sujet pendant ses deux grossesses et pensait que cela pouvait être important pour d'autres femmes diabétiques de trouver facilement des informations de qualité à ce sujet.

Ces attentes ne sont pas concordantes avec les résultats de l'étude ENTRED 2007-201 ${ }^{38}$ qui rapporte que les patients diabétiques de type 1 souhaitent le plus souvent des informations sur les droits et le remboursement des soins (45\%), sur les complications possibles (29\%) et sur la sexualité (21\%). Une des hypothèses pour l'expliquer est que l'échantillon était constitué de personnes dont le diabète avait été diagnostiqué depuis peu (10 mois en moyenne). En moyenne les patients de l'étude ENTRED 2007-2010 étaient âgés de 42 ans et connaissaient l'existence de leur diabète depuis 17 ans. Ils avaient vraisemblablement des attentes différentes.

\subsubsection{La pédagogie}

Le site INDIGO possède les éléments nécessaires à une pédagogie de qualité. Les patients sont tous d'accord pour dire qu'il est illustré par des exemples, que les explications sont soulignées par des schémas et que le vocabulaire est accessible.

Les patients ont apprécié son caractère original et ludique et l'ont trouvé attractif.

La souplesse du support, qui peut s'adapter au rythme de chacun, offre une flexibilité dans la progression et l'apprentissage. 
Enfin l'interactivité permise par le support rend les patients actifs, ce qui est important en matière de pédagogie. ${ }^{39}$

L'utilisation du site INDIGO par les patients correspond à ce que l'on appelle l'autoapprentissage. Il s'agit d'une forme d'enseignement assisté par ordinateur (EAO) également appelée e-learning ou apprentissage en ligne qui est définie comme «une utilisation des nouvelles technologies multimédias et de l'Internet pour améliorer la qualité de l'apprentissage en facilitant l'accès à des ressources et à des services ». ${ }^{40}$

Selon d'Ivernois et Gagnayre, le fait que le patient apprenne seul ne diminue en rien le rôle de l'éducateur. L'auto-apprentissage doit être guidé par l'éducateur et ne représente qu'un complément de l'éducation. Il ne peut en aucun cas être utilisé pour toute l'éducation du patient. ${ }^{5}$ Lacroix et Assal précisent que l'éducateur doit évaluer régulièrement les connaissances du patient afin de s'assurer qu'il ne passe pas trop vite à l'étape suivante et qu'il a acquis les connaissances prévues. ${ }^{41}$

L'éducation comprend une rencontre humaine. Les moyens de communication à distance n'évitent pas cette nécessité. ${ }^{22}$

Il semble en effet indispensable que le patient reformule avec ses propres mots ce qu'il a saisi afin qu'il n'y ait aucune ambiguïté sur ce qui a été compris et qu'une réévaluation régulière de ses savoirs soit effectuée.

De leur côté, tous les patients interrogés envisagent de discuter du site INDIGO avec la personne qui les a informés de l'existence du site, c'est-à-dire leur diabétologue. Ceci semble positif car cela signifie que les informations ont suscité leur intérêt et qu'ils se sont sentis concernés d'une part et cela évitera le risque d'une utilisation isolée d'INDIGO d'autre part. Certains patients ont reconnu la possibilité d'imprimer des documents comme facilitatrice de cette démarche car ceux-ci peuvent constituer un support de discussion avec leur diabétologue.

\subsubsection{Les vidéos de témoignages}

Les vidéos de témoignages sont également un élément important de pédagogie, par les propos qu'elles relayent d'une part et grâce à l'identification qui est permise d'autre part. Quelle que soit l'ancienneté de leur diabète, les patients sont sensibles à cette forme de partage d'expérience et d'apprentissage.

Malgré tout, certains patients les ont parfois trouvées longues ou troublantes mais elles n'ont jamais été signalées comme un frein au visionnage de l'outil car la possibilité de passer la séquence est toujours offerte. 
Au niveau de la forme, la mise en scène et le jeu d'acteur ont été perçus dans certaines séquences comme excessifs voire comiques. A plusieurs reprises les patients ont signalé avoir été moins sérieux et moins concentrés pour les visionner. Cela a pu entrainer une perturbation de la compréhension de l'information ou de l'apprentissage. En conséquence, il parait souhaitable d'envisager une amélioration des passages en question.

Certains patients se sont demandés si les témoins étaient réellement des personnes diabétiques ou s'il s'agissait d'acteurs. Ils ont toutefois précisé que cela ne perturbait pas la transmission du message. Un patient a suggéré de préciser cet élément et de donner un complément d'information sur les témoins. Cette proposition permettrait de lever toute ambiguité et de situer le témoin dans son contexte en précisant des données telles que l'ancienneté de son diabète. Il parait en effet nécessaire que les patients puissent avoir une idée plus précise de la personne qui leur délivre le message.

\subsubsection{Le choix d'Internet}

L'utilisation du support Internet constitue un autre point fort de cet outil d'ETP. Cela le rend disponible au plus grand nombre, au domicile même des patients et à tout moment de la journée. En effet, selon l'INSEE, 78,2\% des plus de 15 ans résidants en France métropolitaine disposaient d'Internet à leur domicile en 2012. Ces chiffres montent à $96,4 \%$ chez les $15-29$ ans et $91,9 \%$ chez les $30-44$ ans. ${ }^{25}$

Toutefois l'étude ENTRED 2007-2010 révélait que le support Internet n'était pas la source d'information de premier choix pour les patients diabétiques de type 1 . Sur les $79 \%$ de personnes diabétiques de type 1 qui déclaraient s'être renseignés d'eux-mêmes sur leur maladie, $77 \%$ avaient eu recours à des livres ou à des documents écrits, bien avant d'utiliser Internet $(42 \%){ }^{2}$

Il faut d'abord noter qu'en 2007, selon l'INSEE, l'accès à Internet était moins répandu (54 $\%$ en 2007 contre 78,2\% en 2012). ${ }^{25}$ D'autre part, dans notre étude, certains patients ont confié leurs craintes de trouver sur Internet des informations inadaptées à leur cas et peu fiables, contrairement aux informations du site INDIGO qui est «validé » par leur diabétologue. On peut formuler l'hypothèse qu'au-delà de l'accès à Internet, la qualité des informations trouvées peut être un frein à l'utilisation d'Internet comme source d'information sur le diabète de type 1 . L'outil INDIGO vient répondre à ce manque de confiance des patients en la fiabilité des informations qui leur sont délivrées sur Internet.

Le choix de ce support expose aussi à un certain nombre de points faibles comme des temps de chargement parfois longs ou des « bugs » techniques. 
Le fait que le site INDIGO ne soit pas disponible sur tous les navigateurs Internet peut se révéler être un frein à son utilisation car le patient peut devoir télécharger le navigateur requis si il ne le possède pas. Il parait donc nécessaire de développer son accessibilité sur tous les navigateurs.

\subsubsection{Le support technique}

La navigation a été appréciée dans l'ensemble, même si certains ont suggéré de limiter le retour au menu entre les chapitres en proposant un accès direct au chapitre suivant ou encore d'augmenter la taille des séquences à l'intérieur des chapitres, la trop grande fragmentation des chapitres provoquant une rupture dans le rythme.

Malheureusement l'étude ne nous a pas permis de tester le site chez des patients ayant une moindre expérience d'Internet, les patients interrogés se définissant tous comme des utilisateurs réguliers ou inconditionnels. Il semble pourtant important d'évaluer l'aisance avec laquelle des personnes moins habituées à Internet et au multimédia vont utiliser l'outil.

Les voix off ont parfois été signalées comme un point négatif du site. Elles ont été considérées comme peu agréables par trois des patients (27\%). Aucun n'a cependant indiqué que cela l'avait conduit à stopper le visionnage. Il paraît difficile de trouver une voix off faisant l'unanimité. Cette évolution ne sera donc pas forcément retenue.

La patiente $\mathrm{n}^{\circ} 12$ aurait souhaité pouvoir trouver indépendamment toutes les informations par écrit ; celles délivrées dans les vidéos, par la voix off et celles apparaissant à l'écran ; de manière à parcourir le site plus vite. Il se peut que cette attente vienne de sa plus «longue » expérience du diabète, les notions principales étant déjà assimilées. Ses besoins sont donc peut-être plus de l'ordre du complément d'information que d'une recherche exhaustive des notions à connaitre lorsque l'on est diabétique de type 1 .

La forme du site INDIGO constitue pourtant l'un de ses points forts essentiels. En effet d'après les données de la littérature, un individu est capable de retenir en moyenne $10 \%$ des informations lues, 20 à $30 \%$ des informations entendues et $30 \%$ de ce qui est vu. Si l'information visuelle est associée à une explication orale, le pourcentage s'élève à $50 \%{ }^{42}$ Il est donc important de combiner les séquences faisant appel à la mémoire visuelle et auditive au cours de l'éducation.

On pourrait cependant envisager la création d'un lien vers un document où toutes les informations apparaitraient sous forme écrite et qu'il serait possible d'imprimer afin qu'il 
serve de support de discussion lors de la consultation suivante. Cela le rendrait par ailleurs accessible aux patients malentendants.

\subsection{Chapitre 1 : «Mes objectifs glycémiques »}

Trois patientes ont dit s'être senties soulagées par ce chapitre en découvrant que leurs objectifs glycémiques ne leur convenaient pas au quotidien et qu'elles pouvaient envisager des objectifs moins restrictifs. Une patiente au contraire a retenu que certaines personnes avaient des objectifs plus stricts qu'elle et cela l'a mise mal à l'aise. Elle s'est dit qu'elle pouvait faire mieux dans la gestion de son diabète.

On s'aperçoit que la même séquence peut provoquer deux ressentis opposés chez les patients.

Il n'y a pas de modérateur lors du visionnage. Le patient est seul face à l'écran et reçoit des informations qui peuvent être source d'angoisse si elles sont mal comprises ou mal interprétées. Cela souligne l'importance du fait que les patients puissent rediscuter du site INDIGO avec le professionnel qui le leur a présenté.

\subsection{Chapitre 2 : «Mes hypos, mes hypers »}

Les sous-chapitres du chapitre 2 ont été diversement appréciés.

Plusieurs patients ont souligné le fait que le sous-chapitre 2.1 « Comment savoir où j'en

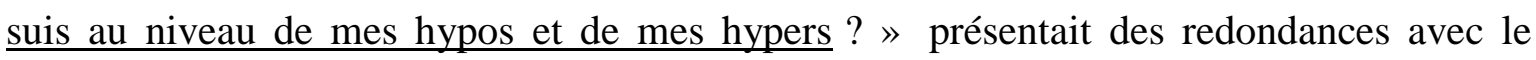
chapitre 1.

Il existe dans le chapitre 1 une séquence où l'on demande aux patients, sous forme de cases à cocher, de s'interroger sur la façon dont ils vivent leurs hypoglycémies et leurs hyperglycémies. Le but est de leur permettre de visualiser les sensations, émotions ou conséquences de celles-ci, parfois difficiles à vivre au quotidien, afin qu'ils fixent leurs objectifs de diminution de fréquence de leurs variations glycémiques.

Dans le chapitre 2, les patients doivent cocher dans une liste leurs symptômes d'hypoglycémies et d'hyperglycémies afin de mieux les identifier pour les prendre en charge.

Bien que semblables dans leur forme et abordant toutes deux les variations glycémiques, ces deux parties proposent des items différents et poursuivent des buts distincts.

Pour mieux les distinguer peut-être faudrait-il en modifier le titre, la forme ou introduire de façon plus claire chaque séance. 
Certains patients ont reproché au chapitre 2 le manque d'informations délivrées sur le traitement des hyperglycémies. Peut-être faudrait-il renommer le sous-chapitre 2.2 «Comment je traite mes hypers et mes hypos?». Le traitement des hyperglycémies est en effet abordé dans le chapitre 3 «Mes insulines » et la voix off et un texte à l'écran renvoient les patients à ce chapitre.

\subsection{Chapitre 3 : «Mes insulines »}

Les informations recherchées par les patients dans ce chapitre sont légèrement différentes en fonction de l'ancienneté de leur diabète. Les patients diagnostiqués plus récemment semblent plus à la recherche d'informations pratiques sur les insulines (telles que les sites d'injections de l'insuline ou comment conserver son insuline en dehors de chez soi). Les patients dont le diabète est plus ancien ayant assimilé ces notions semblent chercher davantage des informations plus précises sur les insulines (telles que les notions de cinétique de l'insuline).

\subsection{Appréciation générale}

Les patients ont attribué une note moyenne de satisfaction globale de $\mathbf{8 , 8 / 1 0}(8,7 / 10$ si l'on inclut la patiente $\mathrm{n}^{\circ} 12$ ). Ils ont considéré que le site INDIGO avait répondu à leurs besoins avec une note moyenne de $\mathbf{8 , 6 / 1 0}$ (8,5 si l'on inclut la patiente $\left.\mathrm{n}^{\circ} 12\right)$.

Si l'on compare le groupe « diagnostiqués depuis moins de $\mathbf{1}$ an » composé des patients $1,2,3,4,6,7,8,9$ et 10 au groupe « diagnostiqués depuis plus de $\mathbf{1}$ an » composé des patients 5,11 et 12 , on ne retrouve pas de différence dans leurs appréciations puisque à la question « Sur une échelle entre 0 à 10 , le site INDIGO a-t-il répondu à vos besoins $?$ » le groupe « diagnostiqués depuis moins de 1 an » a attribué la note moyenne de 8,6/10 contre 8,5/10 pour le groupe «diagnostiqués depuis plus de 1 an ». A la question « $\underline{\text { Sur une }}$ échelle de 0 à 10, quelle est votre satisfaction globale vis-à-vis d'INDIGO ? » les deux groupes ont attribué la note moyenne de 8,7/10.

Si l'on compare le groupe « ETP antérieure » composé des patients 2, 5, 7, 10, 11, 12 au groupe «pas d'ETP antérieure » composé des patients 1, 3, 4, 6, 8 et 9, on ne retrouve pas de différence dans leurs appréciations puisque à la question « $\underline{\text { Sur une échelle entre } 0 \text { à }}$

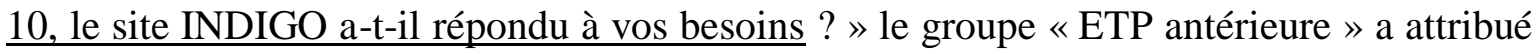
la note moyenne de 8,4/10 contre $8,7 / 10$ pour le groupe «pas d'ETP antérieure ». A la 
question « $\underline{\text { Sur une échelle de } 0 \text { à } 10 \text {, quelle est votre satisfaction globale vis-à-vis }}$ d'INDIGO ? » les deux groupes ont attribué la note moyenne de 8,8/10.

Quelle que soit l'ancienneté de leur diabète et qu'ils aient bénéficié ou non d'ETP antérieurement, les patients interrogés ont trouvé dans le site INDIGO des informations adaptées à leurs besoins et le site, tant au niveau du fond que de la forme, leur a donné globalement satisfaction.

\subsection{Comment utiliser le site INDIGO ?}

Si l'on s'intéresse à la façon dont les patients ont visionné le site INDIGO, on s'aperçoit que la majorité d'entre eux l'a regardé en entier (64\%), en plusieurs fois (55\%), dans l'ordre proposé des chapitres et des sous-chapitres (64\%), seul (74\%) et pense y retourner ultérieurement (100\%). La patiente $n^{\circ} 12$, elle, l'a regardé partiellement. Elle l'a visionné en plusieurs fois et dans l'ordre proposé. Elle l'a regardé seule. Elle a déclaré qu'elle y retournerait pour voir les éléments qu'elle n'avait pas vus.

Le caractère « modulable » du visionnage apparait comme un point fort essentiel du site INDIGO. Les patients ont en effet la possibilité de choisir si ils veulent voir toutes les informations ou non. Ils peuvent aussi le consulter dans l'ordre qu'ils ont choisi et ceci d'autant plus que le site est structuré par un plan pour permettre d'accéder facilement à des éléments précis. Il leur permet d'organiser et de gérer leur apprentissage. Ils peuvent l'utiliser en fonction de leurs besoins et de leur temps disponible.

Le site INDIGO correspond aux critères de qualité d'un processus éducatif qui doit être structuré tout en étant suffisamment souple pour être adapté à chaque patient, à ses besoins, à chaque moment de la prise en charge et en fonction des événements intercurrents. ${ }^{5}$

Les patients interrogés nous ont appris avoir fait leurs choix de visionnage en fonction de leur stade d'avancement dans la maladie, en fonction de leur expérience de leurs symptômes et de leurs traitements, en fonction de leur stade d'acceptation de la maladie et en fonction d'éléments de leur vie personnelle. Ils ont également dit avoir fait leurs choix de visionnage en fonction de leurs besoins éducatifs, de leur intérêt pour tel ou tel sujet et enfin en fonction de leurs acquis antérieurs. Les craintes de ce qu'ils pouvaient entendre dans tel ou tel chapitre ont eu également un rôle dans leur choix.

L'étude ENTRED 2007-2010 ${ }^{2}$ confirme que les besoins éducatifs évoluent selon l'ancienneté de la maladie, son acceptation et l'apparition ou non de complications. 
En effet, «prendre soin de soi »; et non seulement de la maladie et du traitement; est le résultat de processus multiples et dynamiques, de négociations intra- et interpersonnelles en réponse à différentes tensions rencontrées dans la vie avec une maladie chronique : entre des objectifs de santé physique ou biologique et des objectifs de santé psychique et sociale, entre des priorités de vie pour soi ou pour autrui, entre différentes images de soi, et entre différents types de besoins : sécurité, auto-détermination, auto-efficacité, cohérence. $^{43}$

Les patients ont regardé en majorité le site INDIGO en plusieurs fois car ils l'ont trouvé dense. $80 \%$ des patients qui l'ont vu en une fois ont trouvé ça fastidieux. Plusieurs patients nous ont rapporté avoir eu des difficultés de concentration en fin de journée.

Les données de la littérature nous apprennent que pour qu'un enseignement soit efficace, il faut respecter certaines règles et notamment que les séances éducatives doivent durer moins de 45 minutes et qu'elles doivent se dérouler en début de journée de préférence, afin que le patient soit disponible et attentif. ${ }^{5}$

La plupart des patients a déclaré vouloir revenir sur le site au moment de questions particulières. Cette possibilité semble être un autre point fort du site INDIGO. Tout d'abord parce que comme nous l'avons vu les besoins éducatifs évoluent avec le temps. Mais aussi parce que les besoins éducatifs du patient par rapport à sa maladie et à son traitement ne se prêtent pas nécessairement au rythme proposé par le soignant. Le besoin de compréhension du patient, de savoir que faire devant une situation précise peut apparaître bien avant le rendez-vous. Il peut appeler à une réponse relativement immédiate. Il y a donc possibilité de décalage entre les rythmes patients/soignants, entre le jour choisi et le besoin du patient. ${ }^{22}$ La possibilité pour le patient de pouvoir se référer librement au site INDIGO peut alors s'avérer utile, ce que certains patients ont souligné.

\subsection{A quel moment utiliser le site INDIGO ?}

L'objectif de cette étude n'était pas de déterminer quel public de patients diabétiques bénéficierait davantage du site INDIGO. Cependant lors des entretiens semi-dirigés cela a été évoqué avec plusieurs patients. Ceux-ci étaient alors enclins à penser que ce site devrait être proposé en priorité au moment du diagnostic. Ceci vient corroborer les résultats de l'enquête DIABASIS ${ }^{44}$ réalisée auprès de personnes diabétiques de type 2 , selon laquelle le moment du diagnostic est une période où les patients ressentent à la fois un besoin important et un manque d'information. Le patient diabétique pourrait alors retrouver chez 
lui les mêmes éléments que pendant son hospitalisation ainsi que des éléments complémentaires et pourrait se sentir rassuré et moins seul dans la gestion de sa maladie.

Selon les données de la littérature, on pourrait également envisager d'informer les patients de l'existence du site INDIGO à la fin d'un stage d'éducation thérapeutique. D'après Lacroix et Assal, à son retour à domicile, le patient peut avoir oublié certaines notions évoquées durant la séance éducative. Le retour à domicile peut alors être une source d'anxiété pour certains patients qui trouvent une sécurité dans le fait de disposer de documentation reprenant de manière simple les connaissances acquises durant l'éducation. ${ }^{45}$

Enfin le site INDIGO peut également être présenté à des patients dont le diagnostic de diabétique est plus ancien afin de participer au maintien de leurs connaissances.

\subsection{Le site INDIGO et l'entourage des patients diabétiques de type 1}

La nature de l'outil INDIGO le rend facilement accessible à l'entourage des patients. Plusieurs patients ont estimé que le site pourrait rassurer les membres de leur entourage. En effet, ils ont le sentiment que le diagnostic de diabète a été une source d'angoisse pour ces derniers. Plusieurs études confirment que le diagnostic de diabète est une source d'anxiété importante pour les proches. ${ }^{46,}{ }^{47}$ Pour les patients interrogés, les témoignages mettant en scène des patients qui semblent vivre «normalement » avec le diabète (avoir des activités sportives, une activité professionnelle...) pourrait les aider à dédramatiser.

Ensuite les patients ont évoqué la nécessité pour leur entourage d'acquérir certaines connaissances sur le diabète, notamment pour la gestion des situations d'urgence.

L'étude EDUCATED $2^{48}$ a montré un défaut d'éducation de l'entourage des patients diabétiques en France concernant les hypoglycémies. Elle a été menée auprès de l'entourage de patients diabétiques de type 1 ou diabétiques de type 2 traités par insuline. $50 \%$ des membres de l'entourage ne savaient pas reconnaître les signes d'hypoglycémie. $38 \%$ ne savaient citer aucun signe d'hypoglycémie et $77 \%$ disaient ne pas savoir utiliser le Glucagon. L'outil INDIGO peut former l'entourage à ces notions, ainsi qu'à d'autres.

Enfin il faut souligner l'importance du soutien familial dans la gestion du diabète. Plusieurs études ont montré que l'entourage du patient avait un rôle important à jouer dans l'observance du traitement du diabète. Une étude qualitative menée sur des patients 
diabétiques de type 2 adultes $^{49}$ a conclu que les patients observants avaient une famille plus soudée, plus impliquée, plus vigilante et plus attentive. Les membres de la famille lorsqu'ils sont impliqués encouragent le patient à suivre les recommandations, à mettre en place les règles d'hygiène de vie et essayent de les partager. Le site INDIGO semble être un bon outil pour sensibiliser l'entourage aux différentes problématiques du diabète.

\subsection{Au total}

D'après les données collectées auprès des patients lors de cette étude, les consignes d'utilisation à fournir aux patients pourraient donc être :

-Ne pas regarder la totalité du site INDIGO en une seule fois

-Préférer des séances de visionnage de moins de 45 minutes, plutôt le matin -Il n'y a pas d'obligation à regarder le site INDIGO dans l'ordre défini

-Une utilisation régulière peut être envisagée, au gré des besoins, à l'occasion de questions particulières

-Prévoir un retour avec son médecin, prendre en note ses questions.

On pourrait envisager d'éditer un guide d'utilisation à destination des patients que les médecins pourraient fournir au patient en complément d'une information orale adaptée aux besoins de chacun.

\subsection{A l'avenir...}

Il faudrait envisager d'apporter au site INDIGO les améliorations suggérées par cette étude.

Un travail d'évaluation similaire auprès d'endocrinologues, de médecins généralistes et d'autres professionnels de santé et d'ETP devrait être effectué afin de compléter cette étude. Il serait souhaitable de se baser sur le référentiel des critères de qualité d'un outil d'intervention en éducation pour la santé de l'INPES ${ }^{28}$, adapté à la population médicale cette fois et de prévoir des entretiens semi-dirigés.

Il faudrait également s'intéresser aux différents moyens de diffuser le site INDIGO.

Il faudrait enfin envisager, après une diffusion du site à grande échelle, une étude d'impact pédagogique, biomédical et/ou psychosocial. 


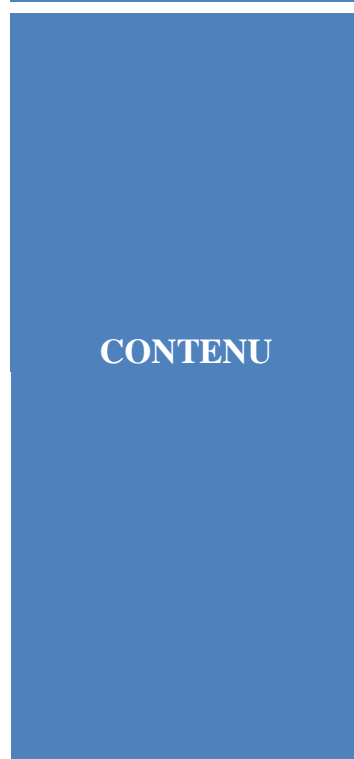

\section{Enrichir le contenu du chapitre 3 « Mes insulines » :}

-Apporter des précisions sur les risques de l'hyperglycémie avec acétone -Informer sur la conduite à tenir en cas d'inversion d'insuline rapide et lente -Informer sur la conservation et le transport des insulines

-Illustrer ce qu'est une lipodystrophie

-Expliquer comment lire une bandelette urinaire

-Donner plus d'informations sur la pompe à insuline ou envisager un chapitre à part entière comparant pompe à insuline et injections au stylo

\section{Créer des chapitres supplémentaires :}

-Epidémiologie et physiopathologie du diabète de type 1

-La recherche dans le domaine du diabète de type 1

-Gestion du diabète de type 1 en voyage

-Vivre avec une maladie chronique

-Diabète de type 1 et grossesse

-Pompe à insuline versus injections au stylo

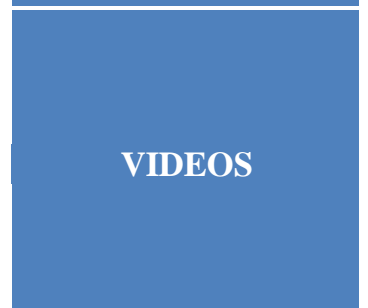

-Donner des informations sur les témoins : préciser si ce sont des patients diabétiques ou des acteurs ; préciser l'ancienneté de leur diabète

-Moderniser la réalisation des vidéos, les rendre moins statiques, plus rythmées -Revoir la mise en scène et le jeu d'acteur des vidéos : «Quel temps accordé pour remplir le carnet INDIGO dans ma vie quotidienne ? » et « J'ai des problèmes d'hypos sévères »

-Rendre le site INDIGO disponible sur tous les navigateurs Internet -Améliorer les temps de chargement des pages et des vidéos -Laisser la possibilité de poursuivre le visionnage avec le chapitre suivant sans retour au menu

SUPPORT TECHNIQUE

- Diminuer le découpage des sous-chapitres en augmentant la durée des séquences - Laisser la possibilité d'avancer et de reculer dans les chapitres sans quitter le mode plein écran

-Mentionner sur la page d'accueil que le site fonctionne avec de l'audio -Créer un lien vers un document imprimable où toutes les informations du site seraient accessibles sous forme de texte

IMPRESSION

-Signaler dans le menu les documents à imprimer avec un lien vers ceux-ci -Présenter le carnet INDIGO au format A4 pour l'impression

GUIDE D'UTILISATION

DIFFUSION -Créer un guide d'utilisation à remettre au patient en même temps qu'une information orale adaptée aux besoins de chacun

-Diffuser le site INDIGO auprès des professionnels de santé, des patients et de leur entourage

-Evaluer le site INDIGO auprès de professionnels de santé et d'experts en ETP

ANALYSES -Mener une analyse d'impact

Tableau 6 : Tableau récapitulatif des propositions faites par les patients (en bleu) et des perspectives envisagées par les soignants (en vert) 
V-Conclusion 
THESE SOUTENUE PAR : Carole KNAPP

TITRE : INDIGO, outil multimédia d'éducation thérapeutique destiné à des patients diabétiques de type 1 adultes : évaluation du point de vue des patients utilisateurs

\section{CONCLUSION}

En positionnant le patient au centre de la démarche de soins, l'éducation thérapeutique du patient est un processus indispensable à une prise en charge optimale du diabète de type 1. Elle permet au patient d'acquérir et/ou de maintenir les connaissances et les compétences nécessaires à la gestion de sa vie avec la maladie chronique et de "prendre soin » de lui dans le but d'améliorer sa qualité de vie et de prévenir la survenue de complications.

La loi HPST inserit l'éducation thérapeutique du patient dans le Code de la Santé Publique comme un droit pour tout patient atteint d'une maladie chronique. Cependant, les offres éducatives existantes ne permettent pas à l'ensemble des patients d'accéder à cette proposition du fait de difficultés d'accessibilité, liées notamment à des contraintes de temps et d'éloignement géographique.

La démocratisation de l'utilisation domestique d'Internet permet d'envisager le multimédia comme support d'éducation thérapeutique du patient.

Dans ce contexte, le service de diabétologie du Centre Hospitalicr Universitaire de Grenoble a créé l'outil INDIGO (Intégrer les Notions essentielles sur le Diabète, l'Insuline et la Glycémie Online), destiné aux patients diabétiques de type 1 et accessible par Internet et sur CD-Rom.

L'objectif principal de cette étude est d'évaluer la qualité du site INDIGO du point de vue des patients utilisateurs selon les critères de qualité d'un outil d'intervention en éducation pour la santé de l'Institut National de Prévention et d'Education pour la Santé. Les objectifs secondaires sont d'ćvaluer ses modalités d'utilisation par les patients, de recueillir leurs appréciations et d'identifier des perspectives d'amélioration.

Une enquête qualitative par entretiens dirigés et semi-dirigés a été menée auprès de onze patients, diabétiques de type 1 adultes, diagnostiqués depuis moins de trois ans, suivis au Centre Hospitalier Universitaire de Grenoble, ayant participé ou non 
à des offres éducatives auparavant. Leurs réponses ont été, de plus, comparées à celles d'une patiente souffrant d'un diabète plus ancien (diagnostiqué depuis 27 ans) afin d'identifier l'impact potentiel de la durée de vécu avec la maladie sur l'utilisation et la satisfaction de l'outil.

Les patients interrogés apprécient la qualité du contenu du site Indigo qu'ils estiment riche, nuancé, fiable et pertinent au regard du thème principal, des objectifs annoncés et du support choisi. La qualité pédagogique est appréciée. Le vocabulaire est jugé accessible, le rythme adapté, le site clairement structuré. L'identification est permise, notamment par les vidéos de témoignages. Les graphiques et les schémas sont mis en avant. La qualité du support est estimée satisfaisante et la navigation agréable et aisée. Sa souplesse d'utilisation est soulignée. En effet, l'outil peut être utilisé en exploitant seulement certaines séquences ce qui le rend adaptable aux besoins de chaque patient. Le choix du support Internet est largement apprécié car il en facilite l'accès, le rend original, attrayant et interactif.

Les difficultés perçues sont des problèmes d'ordre technique comme des temps de chargement parfois longs, des «bugs» informatiques ou l'absence de disponibilité sur tous les navigateurs Internet.

Des conditions d'utilisation optimales semblent se dessiner. Sur la base de ces observations, un guide d'utilisation pourrait être remis par les professionnels médicaux en complément d'une information orale adaptée aux besoins de chacun avec une personnalisation en fonction des situations.

Il semble pertinent de proposer cet outil au patient et à son entourage au moment du diagnostic mais il peut aussi être utilisé ultérieurement par toute personne désireuse d'en savoir plus sur la gestion du diabète de type 1 .

A l'issue de ce travail, plusieurs propositions d'améliorations sont identifiées comme faciliter la navigation entre les chapitres.

Une étude complémentaire pourrait être menée afin d'évaluer la perception des professionnels de santé concernant le site INDIGO et ses modalités d'utilisation dans leur pratique quotidienne de suivi de patients diabétiques de type 1. Puis, après une diffusion à grande échelle, une évaluation de l'impact sur l'évolution des connaissances 
du patient, la prise en charge de sa maladie et l'amélioration de sa qualité de vie devrait être envisagée.

\section{VU ET PERMIS DTMPRIMER}
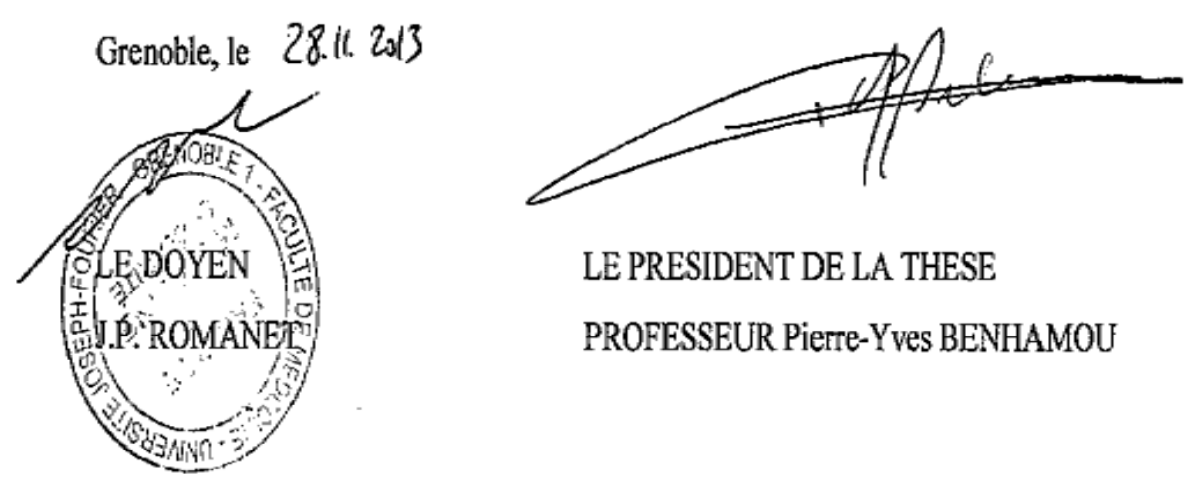

LE PRESIDENT DE LA THESE

PROFESSEUR Pierre-Yves BENHAMOU 
Bibliographie 
1 Institut national de veille sanitaire. Généralités et chiffres clés. Disponible sur: http://www.invs.sante.fr (page consultée le 10/06/2013).

${ }^{2}$ Druet C., Roudier C., Romon I., Assogba F., Bourdel-Marchasson I. et al. Échantillon national témoin représentatif des personnes diabétiques, Entred 2007-2010. Caractéristiques, état de santé, prise en charge et poids économique des personnes diabétiques. Saint-Maurice : Institut de veille sanitaire ; 2013. 140 p. Disponible sur : http://www.invs.sante.fr.

${ }^{3}$ Crozet C., D’Ivernois J.F., Marchand C., Grazia Albano M., Gagnayre R. Recherche en éducation thérapeutique : le patient apprenant. Actes du congrès de l'Actualité de la recherche en éducation et en formation (AREF) ; septembre 2010 ; Université de Genève.

${ }^{4}$ Organisation Mondiale de la Santé. Education thérapeutique du patient : Programme de formation continue pour professionnels de soins dans le domaine de la prévention des maladies chroniques. Recommandations d'un groupe de travail de l'OMS. Bureau régional pour l'Europe, Copenhague ; 1998.

${ }^{5}$ D'Ivernois J.F., Gagnayre R. Apprendre à éduquer le patient, approche pédagogique. 2ème édition. Paris : Maloine ; 2004.

${ }^{6}$ Zito F. Centrer l'éducation du patient sur son projet de vie, incidence sur les pratiques d'une équipe soignante. Education du Patient et Enjeux de Santé. 2007;25:110-115.

${ }^{7}$ Miller L.V., Goldstein J. More efficient use of diabetic patients in a country hospital setting. N Engl J Med 1972;286:1388-91.

${ }^{8}$ Clement S. Diabetes Self-Management Education. Diabetes care, 1995, 18, 8, 1204-1214.

${ }^{9}$ Lagger G., Pataky Z., Golay A. Efficacy of therapeutic patient education in chronic diseases and obesity. Patient Education and Counseling. Juin 2010;79(3):283-286.

${ }^{10}$ Cochran J., Conn VS. Meta-Analysis of quality of life outcomes following diabetes selfmanagement training. Diabetes Educ. 2008;34(5):815.

${ }^{11}$ Ministère de la Santé et des Solidarités. Plan pour l'amélioration de la qualité de vie des personnes atteintes de maladies chroniques 2007-2011; avril 2007. Disponible sur : www.sante.gouv.fr.

${ }^{12}$ Haute Autorité de Santé, Institut National de Prévention et d'Education pour la Santé. Structuration d'un programme d'éducation thérapeutique du patient dans le champ de maladies chroniques. Guide méthodologique. Saint-Denis : HAS ; Juin 2007. Disponible sur : $\underline{\text { www.has-sante.fr. }}$

13 Saout C., Charbonnel B., Bertrand D. Pour une politique nationale d'éducation thérapeutique du patient. Rapport remis au ministre de la santé, de la jeunesse et des sports et de la vie associative ; septembre 2008. Disponible sur : www.sante-sports.gouv.fr.

${ }^{14}$ Loi n ${ }^{\circ} 2009-879$ du 21 juillet 2009 portant réforme de l'hôpital et relative aux patients, à la santé et aux territoires (J.O. 22 juillet 2009). 
${ }^{15}$ Décret $n^{\circ}$ 2010-904 du 2 août 2010 relatif aux conditions d'autorisation des programmes d'éducation thérapeutique du patient (JORF n 0178 du 4 août 2010).

${ }^{16}$ Décret $\mathrm{n}^{\circ}$ 2010-906 du 2 août 2010 relatif aux compétences requises pour dispenser l'éducation thérapeutique du patient (JORF n ${ }^{\circ} 0178$ du 4 août 2010).

17 Arrêté du 2 août 2010 relatif aux compétences requises pour dispenser l'éducation thérapeutique du patient (JORF $n^{\circ} 0178$ du 4 août 2010).

18 Arrêté du 2 août 2010 relatif au cahier des charges des programmes d'éducation thérapeutique du patient et à la composition du dossier de demande de leur autorisation (JORF nº178 du 4 août 2010).

${ }^{19}$ Décret n ${ }^{\circ} 2013-449$ du 31 mai 2013 relatif aux compétences requises pour dispenser ou coordonner l'éducation thérapeutique du patient (JORF n ${ }^{\circ} 0126$ du 2 juin 2013).

${ }^{20}$ Arrêté du 31 mai 2013 modifiant l'arrêté du 2 août 2010 relatif aux compétences requises pour dispenser l'éducation thérapeutique du patient (JORF n0126 du 2 juin 2013).

${ }^{21}$ HAS. Guide méthodologique : Auto-évaluation annuelle d'un programme d'éducation thérapeutique du patient. HAS, mars 2012. Disponible sur : www.has-sante.fr.

22 IPCEM. Questions à propos de l'éducation thérapeutique. [En ligne]. Mai 2013 [Consulté le 08/09/2013]. Disponible sur le site: http://www.ipcem.org.

${ }^{23}$ Gremeaux V. Coudeyre E. The internet and the therapeutic education of patients : a systematic review of the literature. Annals of Physical and Rehabilitation Medicine, 2010;53:669-692.

${ }^{24}$ Balas E.A., Krishna S., Kretschmer R.A., Cheek T.R., Lobach D.F., Boren S.A.. Computerized knowledge management in diabetes care. Med Care. Juin 2004;42(6):610-621.

${ }^{25}$ Gombault V. L'internet de plus en plus prisé, l'internaute de plus en plus mobile. INSEE première. Juin 2013 ; (1452). Disponible sur : http://www.insee.fr.

${ }^{26}$ Leray H., Joubert M., Morera J., Armand C., Tokayeva L., Guillaume A. et al. Utilisation du jeu sérieux "L'affaire Birman" par une population d'enfants et d'adolescents atteints de diabète de type 1 : effet sur l'acquisition de connaissances et de compétences concernant le diabète (étude LUDIDIAB).Conférence SEGAMED. Septembre 2013;Nice.

${ }^{27}$ Benoit M. Conception et réalisation d'un outil éducatif multimédia destiné au patient diabétique de type $1:$ le programme Indigo. Thèse d'exercice en médecine. Grenoble : Université de Grenoble, 2010, 50 p.

${ }^{28}$ Lemonnier F., Bottéro J., Vincent I., Ferron C. Outils d'intervention en éducation pour la santé : critères de qualité. Référentiel de bonnes pratiques. Institut National de Prévention et d'Education pour la Santé ; Novembre 2005. Disponible sur : http://www.inpes.sante.fr/CFESBases/catalogue/pdf/883.pdf.

${ }^{29}$ Gavard-Perret M.L., Aubert B. Méthodologie de la recherche : complément du chapitre 3. Paris : Pearson education ; 2008. 
${ }^{30}$ Blanchet A., Gotman A. L'enquête et ses méthodes - L'entretien. 2ème édition, Armand Colin, Paris, 2011.

${ }^{31}$ Berthier N. Les entretiens. In: N. Berthier. Les techniques d'enquête. Paris : Armand Colin, 1998.

${ }^{32}$ Béaud S., Weber F. Guide de l'enquête de terrain. Paris: Ed. La Découverte, 1998.

33 Bertaux D. L'approche biographique. Sa validité méthodologique, ses potentialités. Cahiers internationaux de sociologie (1980):197-225.

${ }^{34}$ Rogers C. Le développement de la personne. Paris : Dunod. 1996.

${ }^{35}$ Turgeon J., Albert M. La Recherche Qualitative. Synthèse réalisée d'après les ateliers animés par Jean TURGEON Université Laval, Québec et Mathieu ALBERT Université Toronto. Juin 2004.

${ }^{36}$ Loi n ${ }^{\circ} 2002-303$ du 4 mars 2002 relative aux droits des malades et à la qualité du système de santé. JO n54 du 5 mars 2002, p4118.

${ }^{37}$ Institut national de prévention et d'éducation pour la santé. Pédagothèque. Disponible sur http://www.inpes.sante.fr/pedagotheque/presentation.asp (consulté le 18/11/2013).

${ }^{38}$ Fournier C., Gautier A., Attali C., Bocquet-Chabert A., Mosnier-Pudar H., Aujoulat, I. Besoins d'information et d'éducation des personnes diabétiques, pratiques éducatives des médecins, étude Entred, France, 2007. Diabetes Care. 2009 ; 32(1):193-203.

39 Tardif J. Pour un enseignement stratégique. L'apport de la psychologie cognitive. Montréal : Les Editions logiques ; 1992.

${ }^{40}$ Commission des communautés européennes. E-Learning. Penser l'éducation de demain. Bruxelles, 2000.

${ }^{41}$ Lacroix A., Assal J.P. Quelle place pour l'EAO et autres multimédia dans la formation des patients ? In : LACROIX A., ASSAL J.P. L'éducation thérapeutique des patients. Nouvelles approches de la maladie chronique. 2ème édition. Paris : Maloine ; 2003:138146.

${ }^{42}$ Mosnier- Pudar H., Hochberg-Parer G. Education du patient diabétique de type 2. Paris : Phase 5, Editions Médicales ; 2007.

${ }^{43}$ Aujoulat I. L'empowerment des patients atteints de maladies chroniques. Des processus multiples : auto-détermination, auto-efficacité, sécurité et cohérence identitaire (thèse pour le doctorat de santé publique, option éducation du patient. Louvain-la-Neuve : Université catholique de Louvain, 2007:182 p.

${ }^{44}$ Mosnier-Pudar H., Hochberg G., Eschwege E., Virally M.L., Halimi S., Guillausseau P.J. et al. How do patients with type 2 diabetes perceive their disease? Insights from the French DIABASIS survey. Diabetes \& Metabolism. Juin 2009 ; 35(3):220-227. 
${ }^{45}$ Lacroix A., Assal J.P. Prévoir une documentation écrite pour les patients. In : LACROIX A., ASSAL J.P. L'éducation thérapeutique des patients. Nouvelles approches de la maladie chronique. 2ème édition. Paris : Maloine ; 2003:100-102.

${ }^{46}$ Buckloh L.M., Lochrie A.S., Antal H., Milkes A., Canas J.A., Hutchinson S. et al. Diabetes Complications in Youth. Diabetes Care. Août 2008;31(8):1516-1520.

${ }^{47}$ Whittemore R., Jaser S., Chao A., Jang M., Grey M. Psychological experience of parents of children with type 1 diabetes. Diabetes Educ. Juill 2012;38(4):562-579.

${ }^{48}$ Hamdi N. Identification des facteurs prédictifs de l'éducation des patients diabétiques et de leur entourage: Étude ÉDUCATED 2. Thèse de doctorat en médecine. Paris : Université de Paris Diderot-Paris 7, 2012, 111p.

${ }^{49}$ Hochberg G, Dejager S, Eschwege E et al. Observatoire du diabète : Rôle de soutien de l'entourage chez les patients observants mis en évidence dans la phase qualitative. Journal du DELF. Fév. 2008, ${ }^{\circ}$ spécial congrès du 1er février 2008, Abstract nº 10, p14. [Consulté le 23 novembre 2013]. Disponible sur : http://afdet.net/Congres-archives.lasso 
Annexes 


\section{Annexe 1 : Sommaire du site INDIGO}

\section{SOMMAIRE D'INDIGO}

\section{PRÉSENTATION \\ II LES CHAPITRES}

$\Rightarrow \quad$ Chapitre 1 : Mes objectifs glycémiques

$\Rightarrow \quad$ Chapitre 2: Mes hypos, mes hypers

$\Rightarrow$ Chapitre 2.1: Comment savoir où j'en suis au niveau de ma glycémie ?

$\Rightarrow$ Chapitre 2.2 : Comment je traite mes hypers et mes hypos ?

$\Rightarrow$ Chapitre 2.3: J'ai des problèmes d'hypos sévères

$\Rightarrow$ Chapitre 2.4 : Je veux aller plus loin dans l'analyse de l'estimation de ma glycémie

$\Rightarrow$ Chapitre 3 : Mes insulines

$\Rightarrow$ Chapitre 3.1 : Comprendre mon traitement par insuline

$\Rightarrow$ Chapitre 3.2: Mes injections d'insuline

$\Rightarrow$ Chapitre 3.3 : Comment définir mes doses d'insuline ?

$\Rightarrow$ Chapitre 3.4: Le manque d'insuline : hyperglycémie et acétone

$\Rightarrow$ Chapitre 4 : Mon alimentation

$\Rightarrow$ Chapitre 4.1: Combien ai-je de glucides dans mon assiette?

$\Rightarrow$ Chapitre 4.2: Comment prendre en compte l'alcool ?

$\Rightarrow$ Chapitre 4.3 : Comment tenir compte des graisses de mon repas ?

$\Rightarrow$ Chapitre 4.4 : Comment tenir compte de la nature des glucides?

$\Rightarrow$ Chapitre 4.5 : Comment faire si le repas se prolonge?

$\Rightarrow$ Chapitre 4.6: Comment déchiffrer les étiquettes des emballages?

$\Rightarrow$ Chapitre 5 : Mes activités physiques (en cours de réalisation)

\section{LES REFERENCES BIBLIOGRAPHIQUES}

\section{A PROPOS}

$\checkmark$ LES LIENS

VI QUIZ (100 questions/réponses) 

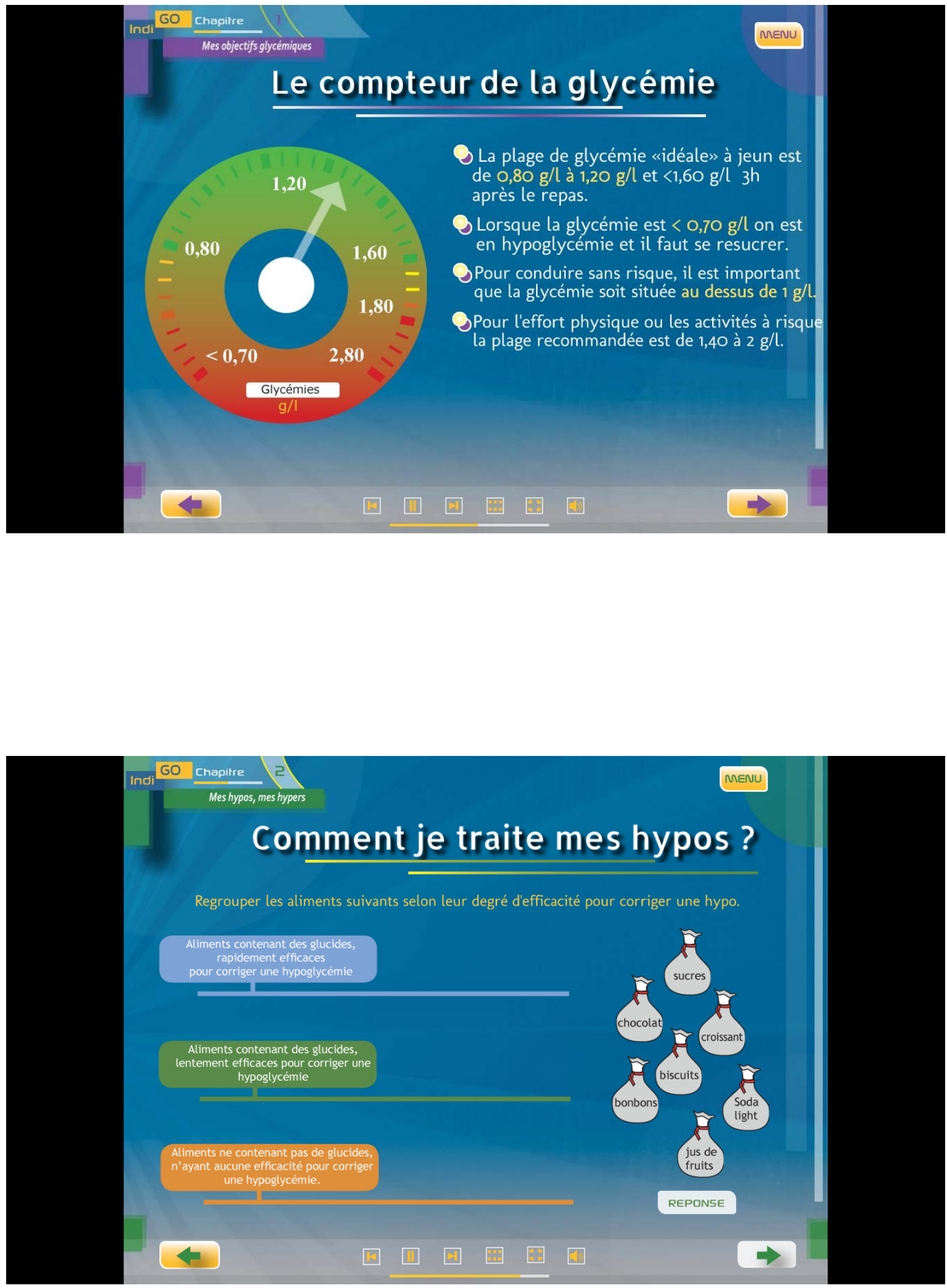

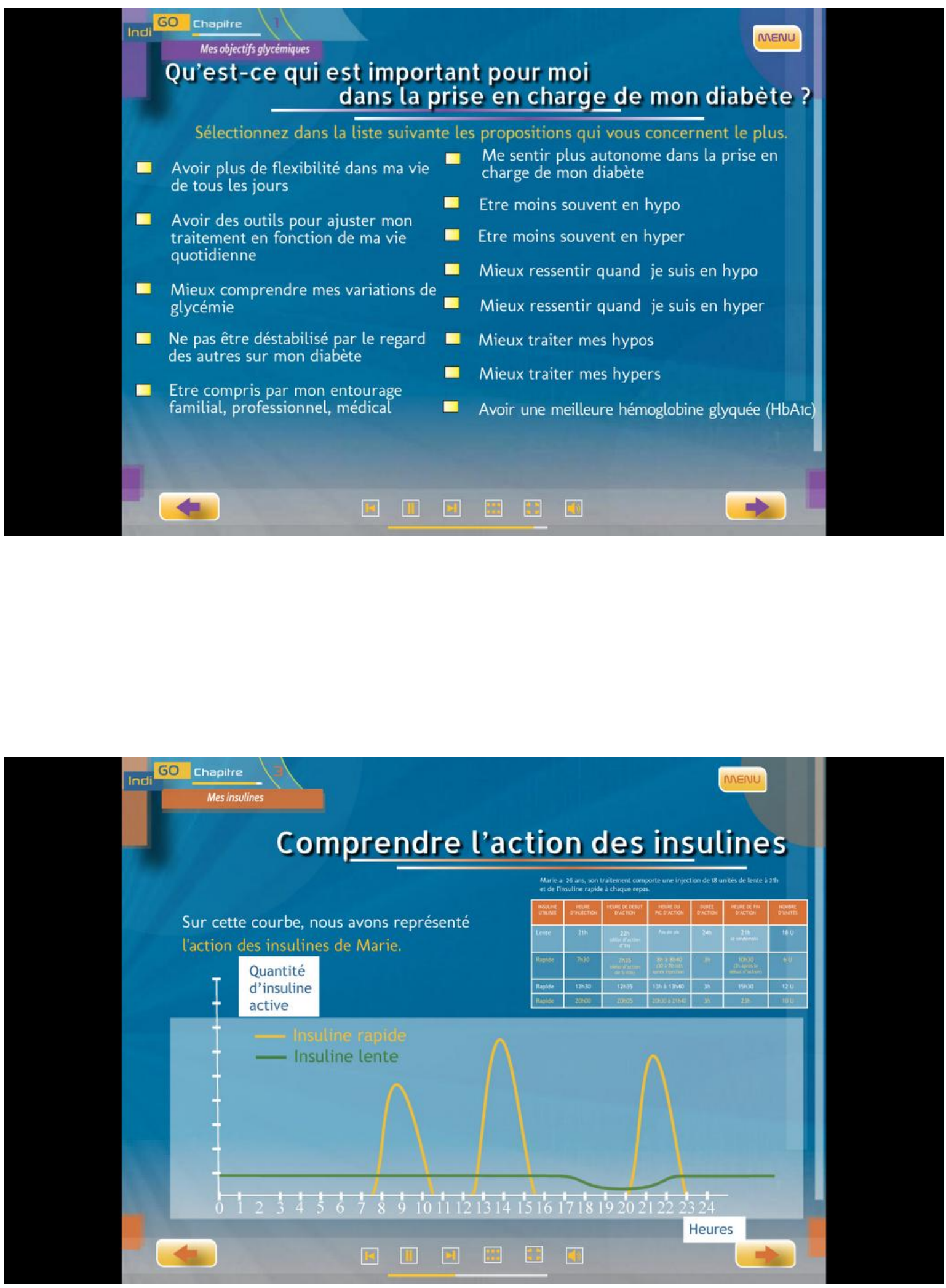


\section{Annexe 3 : Courrier adressé aux patients}

KNAPP Carole

Médecin généraliste

E-mail : carolekn@hotmail.fr

Le 24/08/2013

Madame, Monsieur,

Je réalise actuellement mon travail de thèse sous la direction du Pr Pierre-Yves

BENHAMOU.

Ce travail consiste en une évaluation d'un outil d'éducation thérapeutique original, l'outil Indigo (Intégrer les Notions essentielles sur le Diabète, l'Insuline et la Glycémie Online), destiné aux patients diabétiques de type 1 et accessible par Internet.

\section{Pourquoi cet outil ?}

L'importance de l'éducation thérapeutique du patient diabétique et de son entourage est reconnue par tous les experts. Cependant un grand nombre de patients n'y a pas accès pour diverses raisons (listes d'attente, inégalité de répartition de l'offre sur le territoire, contraintes familiales, contraintes professionnelles...).

Pour tenter de répondre à ce problème, le service de diabétologie du CHU de Grenoble a créé un outil d'éducation thérapeutique qu'il souhaite accessible au plus grand nombre en le rendant disponible sur Internet et sur CD-Rom.

\section{Pourquoi cette étude?}

Cette étude a pour but de recueillir votre opinion concernant la forme et le contenu de cet outil ainsi que vos suggestions d'amélioration. Vos réponses seront le support des améliorations envisagées.

\section{Comment procéder?}

1. Je vous invite dans un premier temps à prendre connaissance du site : http://www.indigo-diabete.fr. Il fonctionne actuellement sur PC et MAC sous les navigateurs Chrome et Safari (pas encore sous Firefox ni Internet Explorer). Vous pouvez télécharger ces navigateurs gratuitement aux adresses suivantes :

http://www.google.ch/chrome?hl=fr et http://support.apple.com/fr_FR/downloads/\#safari.

Le login est : indigo et le mot de passe : glucose.

Les 3 premiers chapitres sont disponibles, les 2 derniers sont en cours de réalisation. Le temps de visionnage complet du site (riche en voix off, images et vidéos) est d'environ 3 heures 30 . Il peut s'effectuer en plusieurs fois.

2. Ensuite, je vous contacterai par téléphone pour prendre rendez-vous pour un entretien si vous acceptez de participer à cette étude. Celui-ci aura lieu en septembre. A votre convenance, il pourra se dérouler à votre domicile, au centre hospitalier universitaire ou encore par téléphone ou par internet via Skype. L'entretien durera environ 30 minutes.

Je vous remercie pour le temps consacré à la lecture de ce document et pour votre participation prochaine je l'espère. 


\section{Annexe 4 : Grille d'analyse des outils d'intervention en éducation pour la santé de l'INPES}

\begin{tabular}{|l|}
\hline Qualité du contenu \\
\hline 1 - Les sources utilisées pour la conception de l'outil sont-elles citées ? \\
\hline 2 - Estimez-vous que l'information délivrée dans l'outil est complète ? \\
\hline 3 - Les informations présentes dans cet outil sont-elles d'actualité ? \\
\hline 4 - Avez-vous eu le sentiment que l'outil faisait la promotion d'un produit ou d'une marque ? \\
\hline 5 - Avez-vous eu le sentiment que l'outil était objectif et nuancé ? \\
\hline 6 - Avez-vous eu le sentiment que l'outil comportait des éléments caricaturaux ou des stéréotypes entraînant \\
une perturbation de la compréhension du contenu? \\
\hline 7 - Avez-vous eu le sentiment que l'outil comportait un contenu inacceptable au regard de l'éthique ? \\
\hline 8 - Quelle est, selon vous, la pertinence du contenu par rapport au thème principal de l'outil ? 0 à 10 \\
\hline 9- Quelle est, selon vous, la pertinence du contenu par rapport aux objectifs annoncés ? 0 à 10 \\
\hline
\end{tabular}

\section{Qualité pédagogique}

Construction pédagogique

10 - Les objectifs de l'outil sont-ils annoncés ?

11 - Diriez-vous que l'émetteur du discours est facilement identifiable?

12 - Diriez-vous que l'émetteur est légitime au regard des propos tenus dans l'outil ?

13 - Le point de vue du destinataire est-il pris en compte ?

14 - Le contenu de l'outil est-il clairement structuré (par un plan ou un sommaire, par exemple) ?

15 - L'outil comporte-t-il des éléments facilitant la compréhension (par exemple des schémas, des graphiques)?

16 - L'outil comporte t-il des éléments facilitant la mémorisation (répétitions, moyens mnémotechniques...) ?

17 - L'outil comporte-t-il des éléments facilitant l'appropriation du contenu (exemples variés, règles...) ?

18 - Diriez-vous que l'outil évite la mise en échec des destinataires ?

19 - L'outil peut-il être utilisé en exploitant seulement certains éléments ou certaines séquences ?

20 - L'outil comporte-t-il une rubrique proposant à l'utilisateur une liste de ressources lui permettant d'aller au-delà du strict contenu de l'outil ?

\section{Implication du destinataire}

21 - Pensez-vous que le niveau de difficulté de l'outil est adapté au destinataire ? Du point de vue du choix du vocabulaire ? Des schémas utilisés ? Des règles du jeu ?

22 - L'identification avec les personnages de l'outil est-elle possible ?

23 - Des exemples sont-ils utilisés dans le cadre de cet outil ? Si oui, sont-ils proches de situations vécues ?

24 - Le destinataire de l'outil est-il : Interpellé ? Rendu actif ? Mis en situation ?

25 - Diriez-vous que les connaissances et les représentations préexistantes des destinataires sont prises en compte?

\section{Implication collective du destinataire}

26 - Diriez-vous de l'outil qu'il propose des activités suscitant des interactions entre participants ?

27 - Diriez-vous de l'outil qu'il propose des activités qui suscitent des interactions avec l'environnement (hors groupe)?

\section{Tonalité de l'outil}

28 - Par son contenu et sa forme, l'outil peut-il susciter des sentiments (malaise, crainte, honte...) produisant un effet négatif ? 0 à 10

29 - Citer les ressorts émotionnels ou intellectuels utilisés par le ou les concepteur(s) de l'outil

30 - Parmi ces ressorts, y en a-t-il qui soient en désaccord avec les valeurs défendues par la promotion de la santé (charte d'Ottawa)?

31 - Parmi ces ressorts, y en a-t-il qui nuisent à l'implication des destinataires ?

Evaluation

32 - L'outil prévoit-il une procédure d'évaluation à appliquer par les utilisateurs auprès des destinataires ?

\section{Qualité du support}

33 - Qualité globale des images animées ? 0 à 10

34 - Qualité des illustrations ? 0 à 10

35 - Lisibilité du support écrit ? 0 à 10

36 - Qualité de l'expression et de l'écriture ? 0 à 10 


\begin{tabular}{|l|}
\hline 37 - Qualité du son ? 0 à 10 \\
\hline 38 - Diriez-vous que cet outil est original, qu'il tranche ou se distingue par rapport aux productions \\
habituelles? \\
\hline 39 - Si oui, cette originalité renforce-t-elle l'implication des destinataires ? \\
\hline 40 - Si oui, cette originalité favorise-t-elle les interactions ? \\
\hline 41 - Qualité des matériaux (solidité et robustesse) ? 0 à 10 \\
\hline 42 - Pertinence du choix du support au regard des destinataires ? 0 à 10 \\
\hline 43 - Pertinence du choix du support au regard du sujet traité ? 0 à 10 \\
\hline 44 - Pertinence du choix du support au regard des objectifs annoncés ? 0 à 10 \\
\hline 45 - Clarté des consignes d'utilisation ? 0 à 10 \\
\hline 46 - Actualité de la forme (vocabulaire, fond sonore, vêtements...) ? 0 à 10 \\
\hline 47 - Y a-t-il un guide d'utilisation ? \\
\hline 48 - Si non, l'absence de guide d'utilisation est-elle un manque ? \\
\hline 49 - Si oui, le guide d'utilisation explique-t-il la ou les méthodes pédagogiques ? \\
\hline 50 - Si oui, le guide d'utilisation inclut-il des ressources documentaires ? \\
\hline 51 - Si oui, le guide d'utilisation inclut-il une description des précautions d'usage (pièges ou problèmes à \\
éviter) ? \\
\hline 52 - Si oui, le guide d'utilisation inclut-il des suggestions pour l'évaluation ? \\
\hline 53 - Si le support est un cédérom : aisance de la navigation ? 0 à 10 \\
\hline 54 - Si le support est un cédérom : facilité d'impression ? 0 à 10 \\
\hline 55 - Si le support est un cédérom : facilité des liens avec Internet ? 0 à 10 \\
\hline 56 - Si le support est un cédérom : compréhension des boutons et icônes ? 0 à 10 \\
\hline 57 - Si le support est un cédérom : logique de l'organisation ? 0 à 10 \\
\hline 58 - Si le support est un cédérom : réalité du caractère interactif ? 0 à 10 \\
\hline
\end{tabular}

\section{Qualité de la conception}

59 - Selon vous, la conception de l'outil s'appuie-t-elle sur une analyse des besoins des destinataires ? 60 - Selon vous, la conception de l'outil s'appuie-t-elle sur une analyse de la littérature ?

61 - Avez-vous le sentiment que le destinataire a été impliqué dans le processus de conception de l'outil ?

62 - Avez-vous le sentiment que des représentants de toutes les disciplines concernées ont été impliqués dans le processus de conception de l'outil?

\section{Appréciation d'ensemble}

63 - Quelle est, selon vous, l'adéquation de l'outil avec les objectifs annoncés/le public destinataire ? 0 à 10 64 - Si l'outil est composé de plusieurs supports, ceux-ci sont-ils cohérents entre eux ?

65 - Si l'outil précise les conditions optimales d'utilisation (nombre de participants, âge des participants, etc.), les conditions d'utilisation initialement prévues par le ou les concepteurs sont-elles pertinentes ? 


\section{Annexe 5 : Questionnaire de l'entretien dirigé}

$\square$ un homme $\square$ une femme

Quel âge avez-vous? ans

Quel est votre niveau d'étude ? $\square$ Brevet $\square$ Baccalauréat $\square$ Bac $+2 \square$ Bac +3 et plus Autre :

Quelle profession exercez-vous?

Avez-vous bénéficié d'une offre d'éducation thérapeutique antérieure ? $\square$ Oui $\quad \square$ Non

Si oui : Nombre de séances : $\underline{\text { Année(s) }}$

$\square$ en groupe $\square$ en séance individuelle $\square$ les deux

$\square$ au cours d'une hospitalisation (programmée ou non) $\square$ en ambulatoire

Sujets abordés :

$\underline{\text { Possédez-vous une connexion Internet à votre domicile }} ? \square$ Oui $\square$ Non Sur votre lieu de

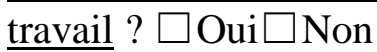

Etes-vous un utilisateur d'Internet et/ou de multimédia ?

$\square$ Occasionnel $\square$ Régulier $\square$ Inconditionnel

Avez-vous déjà cherché des informations en lien avec votre santé sur Internet ?

$\square$ Oui $\square$ Non

Avez-vous déjà cherché des informations sur votre diabète sur Internet ? $\square$ Oui $\quad \square$ Non

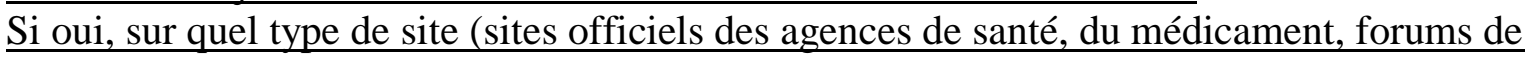
discussion, associations de patients, doctissimo, univadis...) ?

\section{Quelques questions concernant le site Indigo :}

\section{1) Vos appréciations sur la qualité du contenu ?}

Q1 - Estimez-vous que l'information délivrée dans les 3 chapitres disponibles est ?

$\square$ Très complète $\square$ Plutôt complète $\square$ Plutôt incomplète $\square$ Très incomplète

Q2 - Avez-vous eu le sentiment que le site Indigo faisait la promotion d'un produit ou d'une marque?

$\square$ Oui tout à fait d'accord $\square$ Oui plutôt d'accord $\square$ Non, plutôt pas d'accord $\square$ Non, pas du tout d'accord

Q3 - Avez-vous eu le sentiment que le site Indigo apportait des informations nuancées, c'est-à-dire ni exagérées ni sous-estimées ?

$\square$ Oui tout à fait d'accord $\square$ Oui plutôt d'accord $\square$ Non, plutôt pas d'accord $\square$ Non, pas du tout d'accord 
Q4 - Avez-vous eu le sentiment que le site Indigo comportait des éléments caricaturaux ou des stéréotypes qui vous mettaient mal à l'aise ?

$\square$ Non $\square$ Oui, précisez :

Q5 - Avez-vous eu le sentiment que le site Indigo comportait un contenu inacceptable au regard de vos valeurs ? $\square$ Non $\square$ Oui, précisez :

Q6 - Quelle est, selon vous, la pertinence de la présence du chapitre «Mes objectifs glycémiques $»$ ? (Entre 0 et $10,0=$ pas pertinent du tout, $10=$ très pertinent $)$

Q7 - Quelle est, selon vous, la pertinence de la présence du chapitre « Mes hypos, mes

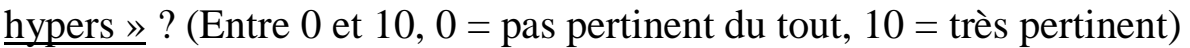

Q8 - Quelle est, selon vous, la pertinence de la présence du chapitre « Mes insulines »? (Entre 0 et $10,0=$ pas pertinent du tout, $10=$ très pertinent)

Q9 - Quelle est, selon vous, la pertinence du contenu du chapitre « Mes objectifs glycémiques » par rapport aux objectifs annoncés (définir votre plage de glycémie « acceptable », analyser où vous en êtes par rapport à cette plage et déterminer vos objectifs de réduction de fréquence de vos hypoglycémies et de vos hyperglycémies) ? (Entre 0 et $10,0=$ pas pertinent du tout, $10=$ très pertinent)

Q10 - Quelle est, selon vous, la pertinence du contenu du chapitre « Mes hypos, mes hypers » par rapport aux objectifs annoncés (gérer les fluctuations de votre glycémie afin que celles-ci ne vous empêchent de vivre pleinement) ?

(Entre 0 et $10,0=$ pas pertinent du tout, $10=$ très pertinent)

Q11 - Quelle est, selon vous, la pertinence du contenu du chapitre « Mes insulines »par rapport aux objectifs annoncés (comprendre ce qu'est l'insuline, son fonctionnement afin de mieux équilibrer vos glycémies au quotidien) ?

(Entre 0 et $10,0=$ pas pertinent du tout, $10=$ très pertinent)

\section{2) Vos appréciations sur la qualité pédagogique?}

Q12 - Le contenu du site Indigo est-il clairement structuré (par un plan ou un sommaire)?

\section{$\square$ Oui $\square$ Non}

Q13 - Le site Indigo comporte-t-il des éléments facilitant la compréhension (par exemple des schémas, des graphiques) ? $\square$ Oui $\square$ Non

Q14 - Le site Indigo comporte-t-il des éléments facilitant la mémorisation (répétitions, moyens mnémotechniques...) ? $\square$ Oui $\square$ Non

Q15 - Le site Indigo comporte-t-il des éléments facilitant l'appropriation du contenu (exemples variés, règles...) ? $\square$ Oui $\square$ Non

Q16 - Vous êtes-vous senti déstabilisé dans la gestion de votre diabète au quotidien par le site Indigo ? $\square$ Oui tout à fait d'accord $\square$ Oui plutôt d'accord $\square$ Non, plutôt pas d'accord $\square$ Non, pas du tout d'accord 
Q17 - Le vocabulaire est-il compréhensible ?

Oui tout à fait d'accord $\square$ Oui plutôt d'accord $\square$ Non, plutôt pas d'accord $\square$ Non, pas du tout d'accord

Q18 - Les schémas utilisés sont-ils faciles à comprendre ?

$\square$ Oui tout à fait d'accord $\square$ Oui plutôt d'accord $\square$ Non, plutôt pas d'accord $\square$ Non, pas du tout d'accord

Q19 - Les exemples utilisés sont-ils proches des situations que vous vivez au quotidien ?

$\square$ Oui $\square$ Non

Q20 - Vous êtes-vous senti ?

Interpellé ?

Très souvent

Souvent Rarement

Jamais

Rendu actif ?

Mis en situation?

Q21 - Le site Indigo a t-il provoqué/va-t-il provoquer des discussions entre vous et ?

\begin{tabular}{|l|l|l|l|l|}
\hline & $\begin{array}{l}\text { Tout à fait } \\
\text { d'accord }\end{array}$ & Plutôt d'accord & Plutôt pas d'accord & Pas du tout d'accord \\
\hline Votre famille & & & & \\
\hline Votre médecin généraliste & & & & \\
\hline Votre diabétologue & & & & \\
\hline Votre pharmacien & & & & \\
\hline Votre infirmière & & & & \\
\hline Votre diététicienne & & & & \\
\hline $\begin{array}{l}\text { Autres professionnels de } \\
\text { santé, lesquels :.............. }\end{array}$ & & & & \\
\hline
\end{tabular}

\section{3) Vos appréciations sur la qualité du support?}

Q22-Que pensez-vous de la qualité des vidéos?

(Entre 0 et $10,0=$ très mauvaise, $10=$ très bonne)

Q23 - Que pensez-vous de la qualité des photos?

(Entre 0 et $10,0=$ très mauvaise, $10=$ très bonne) :

Q24 - Que pensez-vous de la qualité du texte à l'écran (taille, police, couleur) ?

(Entre 0 et $10,0=$ très mauvaise, $10=$ très bonne) $:$

Q25 - Les voix off sont-elles agréables?

(Entre 0 et $10,0=$ pas du tout, $10=$ tout à fait) :

Q26 - Les commentaires sont-ils audibles?

(Entre 0 et 10, $0=$ pas du tout, $10=$ tout à fait) : 
Q27 - Diriez-vous que le site Indigo est original par rapport aux autres documents auxquels vous avez eu accès (brochures, livres, autres sites internet...) ?

$\square$ Oui $\quad \square$ Non

Q28 - Si oui, est-ce cette originalité qui vous a donné envie de le parcourir ?

$\square$ Oui, tout à fait $\square$ Oui, plutôt $\square$ Non, pas du tout $\square$ Non, plutôt pas $\square$ Ne se prononce pas

Q29 - Selon vous, le choix d'utiliser le support internet est-il pertinent pour aborder le thème «Mes objectifs glycémiques » ? (Entre 0 et 10, $0=$ pas pertinent du tout, $10=$ très pertinent) :

Q30 - Selon vous, le choix d'utiliser le support internet est-il pertinent pour aborder le thème «Mes hypos, mes hypers »? (Entre 0 et $10,0=$ pas pertinent du tout, $10=$ très pertinent) :

Q31 - Selon vous, le choix d'utiliser le support internet est-il pertinent pour aborder le thème «Mes insulines » ? (Entre 0 et 10, $0=$ pas pertinent du tout, $10=$ très pertinent) :

Q32 - L'absence de guide d'utilisation est-elle un manque ?

\section{$\square$ Oui $\square$ Non}

Q33 - La navigation est-elle aisée ?

(Entre 0 et $10,0=$ pas du tout, $10=$ tout à fait $)$ :

Q34 - L'impression des documents est-elle facile?

(Entre 0 et $10,0=$ pas du tout, $10=$ tout à fait) :

Q35 - Les boutons et icônes sont-ils compréhensibles ?

(Entre 0 et $10,0=$ pas du tout, $10=$ tout à fait) :

Q36 - L'architecture du site est-elle logique ?

(Entre 0 et $10,0=$ pas du tout, $10=$ tout à fait) :

\section{4) Vos appréciations sur la qualité de la conception?}

Q37 - Avez-vous le sentiment que des patients diabétiques ont été impliqués dans le processus de conception?

$\square$ Non $\square$ Oui, des indices me le font penser $\square$ Oui, car c'est signalé par le ou les concepteur(s) $\square$ Ne se prononce pas

\section{5) Votre appréciation d'ensemble ?}

Q38 - Le site Indigo a-t-il répondu à vos besoins ? (Entre 0 et $10,0=$ pas du tout, $10=$ tout à fait) :

Q39 - Les 3 chapitres disponibles vous ont-ils semblé cohérents entre eux ?

Oui, tout à fait $\square$ Oui, plutôt $\square$ Non, pas du tout $\square$ Non, plutôt pas 


\section{Annexe 6 : Guide d'entretien}

Je vous remercie de m'avoir accordé de votre temps pour échanger ensemble sur le site INDIGO. L'objectif de cet entretien est que vous puissiez me faire le retour de vos impressions sur ce site : son contenu, sa forme, ce que vous avez apprécié ou à l'inverse ce que vous souhaiteriez voir changer. Pour cela je vais vous poser quelques questions mais n'hésitez pas à ajouter des choses si vous pensez à d'autres éléments importants à discuter. La conversation est enregistrée pour recueillir vos propos de la manière la plus exacte possible mais cet entretien est strictement confidentiel et anonyme.

-Quelle serait la première chose que vous auriez envie de me dire après avoir exploré le site INDIGO ?

-Nous allons parler de la façon dont vous l'avez visionné. Racontez-moi.

(Relances : Vous l'avez consulté seul ou avec quelqu'un? L'avez-vous regardé en une fois ? Y êtes-vous revenu plusieurs fois ?)

- Parlez-moi du $1^{\text {er }}$ chapitre.

Thèmes à aborder :

a. Comment l'avez-vous regardé ? (En entier ? Si non, pourquoi ? Dans l'ordre défini ? En une ou plusieurs fois ?)

b. Points forts ? Contenu/Forme

c. Points faibles ? Contenu/Forme. Qu'est-ce que vous attendiez ? Comment pourrait-on l'améliorer?

d. Les informations contenues dans le chapitre ont-elles répondu aux questions que vous vous posiez sur vos objectifs glycémiques?

e. Avez-vous des suggestions d'amélioration?

-Parlez-moi du $2^{\text {ème }}$ chapitre.

Thèmes à aborder : idem

-Parlez-moi du $3^{\text {ème }}$ chapitre.

Thèmes à aborder : idem

-De façon générale :

a. Quel est le chapitre ou sous-chapitre qui vous a semblé le moins intéressant ou répondant le moins à des événements de votre vie quotidienne ?

b. Quel est le chapitre ou le sous-chapitre qui vous a semblé le plus intéressant ou le plus utile pour gérer votre diabète au quotidien ?

c. Quels autres thèmes aimeriez-vous trouver dans le futur ?

-Quelle est votre satisfaction globale vis-à-vis d'INDIGO ? 0 à 10

-Estimez-vous qu'INDIGO vous ait apporté des éléments qui allaient vous aider dans la gestion de votre diabète au quotidien ? Si oui lesquels ?

-Pour terminer, conseilleriez-vous le site INDIGO à une autre personne diabétique ? A un membre de votre entourage ?

-Souhaitez-vous être informé lorsque les chapitres «Mon alimentation » et « Mes activités physiques » seront disponibles? 


\section{Annexe 7 : Notes attribuées par les patients au questionnaire dirigé}

\begin{tabular}{|c|c|c|c|c|c|c|c|c|c|c|c|c|}
\hline & \multicolumn{12}{|c|}{ Note attribuée par le patient $\mathbf{n}^{\circ}$} \\
\hline & 1 & 2 & 3 & 4 & 5 & 6 & 7 & 8 & 9 & 10 & 11 & 12 \\
\hline $\begin{array}{l}\text { Q6 : Quelle est, selon vous, la pertinence } \\
\text { de la présence du chapitre « Mes } \\
\text { objectifs glycémiques »? }\end{array}$ & 10 & 8 & 7,5 & 10 & 8,5 & 8 & 8,5 & 7 & 10 & 10 & 9 & 10 \\
\hline $\begin{array}{l}\text { Q7 : Quelle est, selon vous, la pertinence } \\
\text { de la présence du chapitre « Mes hypos, } \\
\text { mes hypers»? }\end{array}$ & 9,5 & 8 & 9,5 & 10 & 9,5 & 7,5 & 10 & 8 & 8 & 10 & 9 & 9,5 \\
\hline $\begin{array}{c}\text { Q8 : Quelle est, selon vous, la pertinence } \\
\text { de la présence du chapitre « Mes } \\
\text { insulines»? }\end{array}$ & 9 & 9 & 9,5 & 10 & 10 & 7,5 & 9,5 & 8 & 10 & 10 & 9 & 10 \\
\hline $\begin{array}{l}\text { Q9 : Quelle est, selon vous, la pertinence } \\
\text { du contenu du chapitre « Mes objectifs } \\
\text { glycémiques » par rapport aux objectifs } \\
\text { annoncés? }\end{array}$ & 10 & 8 & 5 & 9,5 & 8,5 & 4,5 & 9 & 10 & 10 & 10 & 7 & 9,5 \\
\hline $\begin{array}{l}\text { Q10 : Quelle est, selon vous, la } \\
\text { pertinence du contenu du chapitre } \\
\text { «Mes hypos, mes hypers » par rapport } \\
\text { aux objectifs annoncés? }\end{array}$ & 9,5 & 7 & 7,5 & 9,5 & 10 & 5,5 & 9,5 & 10 & 7 & 10 & 7 & 9,5 \\
\hline $\begin{array}{l}\text { Q11 : Quelle est, selon vous, la } \\
\text { pertinence du contenu du chapitre } \\
\text { « Mes insulines » par rapport aux } \\
\text { objectifs annoncés? }\end{array}$ & 9,5 & 8 & $\begin{array}{l}\text { Sans } \\
\text { objet }\end{array}$ & 9 & 9,5 & 5 & 9,5 & 10 & $\begin{array}{l}\text { Sans } \\
\text { objet }\end{array}$ & 10 & 9 & 9,5 \\
\hline $\begin{array}{l}\text { Q22 : Que pensez-vous de la qualité des } \\
\text { vidéos? }\end{array}$ & 7 & 8 & 9,5 & 9,5 & 7 & 10 & 8 & 7 & 10 & 8 & 7 & 9,5 \\
\hline $\begin{array}{l}\text { Q23 : Que pensez-vous de la qualité des } \\
\text { photos? }\end{array}$ & 8,5 & 8 & 8,5 & 9,5 & 9 & 10 & 7,5 & 7 & 8 & 8 & 5 & 9,5 \\
\hline $\begin{array}{l}\text { Q24 : Que pensez-vous de la qualité du } \\
\text { texte à l'écran (taille, police, couleur)? }\end{array}$ & 9,5 & 4 & 9,5 & 9,5 & 10 & 10 & 5 & 8 & 10 & 10 & 7 & 9,5 \\
\hline Q25 : Les voix off sont-elles agréables? & 9,5 & 8 & 8,5 & 8,5 & 7 & 10 & 7,5 & 8 & 10 & 5 & 5 & $\mathbf{0 , 5}$ \\
\hline $\begin{array}{c}\text { Q26 : Les commentaires sont-ils } \\
\text { audibles? }\end{array}$ & 7,5 & 10 & 9,5 & 9,5 & 10 & 9 & 10 & 7 & 10 & 8 & 7 & 10 \\
\hline $\begin{array}{l}\text { Q29 : Selon vous, le choix d'utiliser le } \\
\text { support Internet est-il pertinent pour } \\
\text { aborder le thème « Mes objectifs } \\
\text { glycémiques»? }\end{array}$ & 9,5 & 7 & 9 & 9,5 & 5,5 & 8 & 5,5 & 7 & 10 & 8 & 8 & 10 \\
\hline $\begin{array}{l}\text { Q30 : Selon vous, le choix d'utiliser le } \\
\text { support Internet est-il pertinent pour } \\
\text { aborder le thème « Mes hypos, mes } \\
\text { hypers »? }\end{array}$ & 9,5 & 8 & 9 & 9,5 & 8,5 & 8,5 & 9,5 & 9 & 7 & 8 & 8 & 10 \\
\hline $\begin{array}{l}\text { Q31 : Selon vous, le choix d'utiliser le } \\
\text { support Internet est-il pertinent pour } \\
\text { aborder le thème « Mes insulines»? }\end{array}$ & 9,5 & 8 & 9 & 9,5 & 10 & 8,5 & 9,5 & 9 & 9 & 8 & 8 & 10 \\
\hline Q33 : La navigation est-elle aisée ? & 9 & 5 & 9,5 & 9,5 & 9 & 7 & 10 & 6 & 10 & 7 & 10 & 1,5 \\
\hline $\begin{array}{l}\text { Q34 : L'impression des documents est- } \\
\text { elle facile? }\end{array}$ & 9,5 & $\begin{array}{l}\text { Sans } \\
\text { objet }\end{array}$ & 9,5 & $\begin{array}{l}\text { Sans } \\
\text { objet }\end{array}$ & 10 & 10 & $\begin{array}{l}\text { Sans } \\
\text { objet }\end{array}$ & $\begin{array}{l}\text { Sans } \\
\text { objet }\end{array}$ & $\begin{array}{l}\text { Sans } \\
\text { objet }\end{array}$ & 10 & 10 & $\begin{array}{l}\text { Sans } \\
\text { objet }\end{array}$ \\
\hline $\begin{array}{c}\text { Q35 : Les boutons et icônes sont-ils } \\
\text { compréhensibles? }\end{array}$ & 9,5 & 8 & 9,5 & 9,5 & 10 & 10 & 9,5 & 7 & 10 & 10 & 10 & 10 \\
\hline $\begin{array}{l}\text { Q36 : L'architecture du site est-elle } \\
\text { logique? }\end{array}$ & 9,5 & 8 & 9,5 & 9,5 & 10 & 10 & 9,5 & 8 & 10 & 10 & 10 & 10 \\
\hline $\begin{array}{c}\text { Q38 : Le site INDIGO a-t-il répondu à } \\
\text { vos besoins? }\end{array}$ & 10 & 8 & 6,5 & 9,5 & 9,5 & 9 & 7 & 7 & 10 & 10 & 8 & 8 \\
\hline
\end{tabular}


Annexe 8 : Grille d'analyse des erreurs

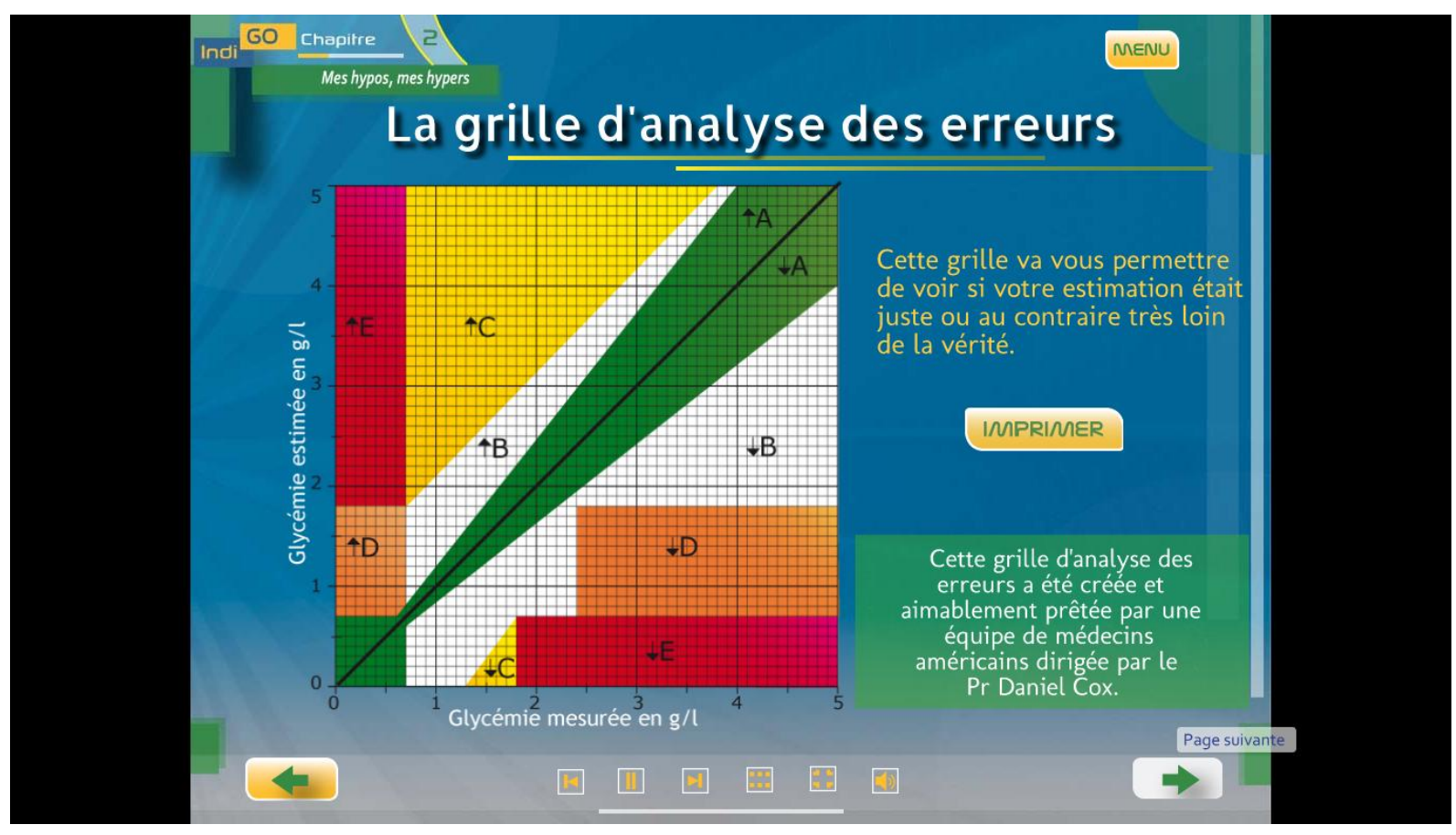

\section{Annexe 9 : Carnet INDIGO}

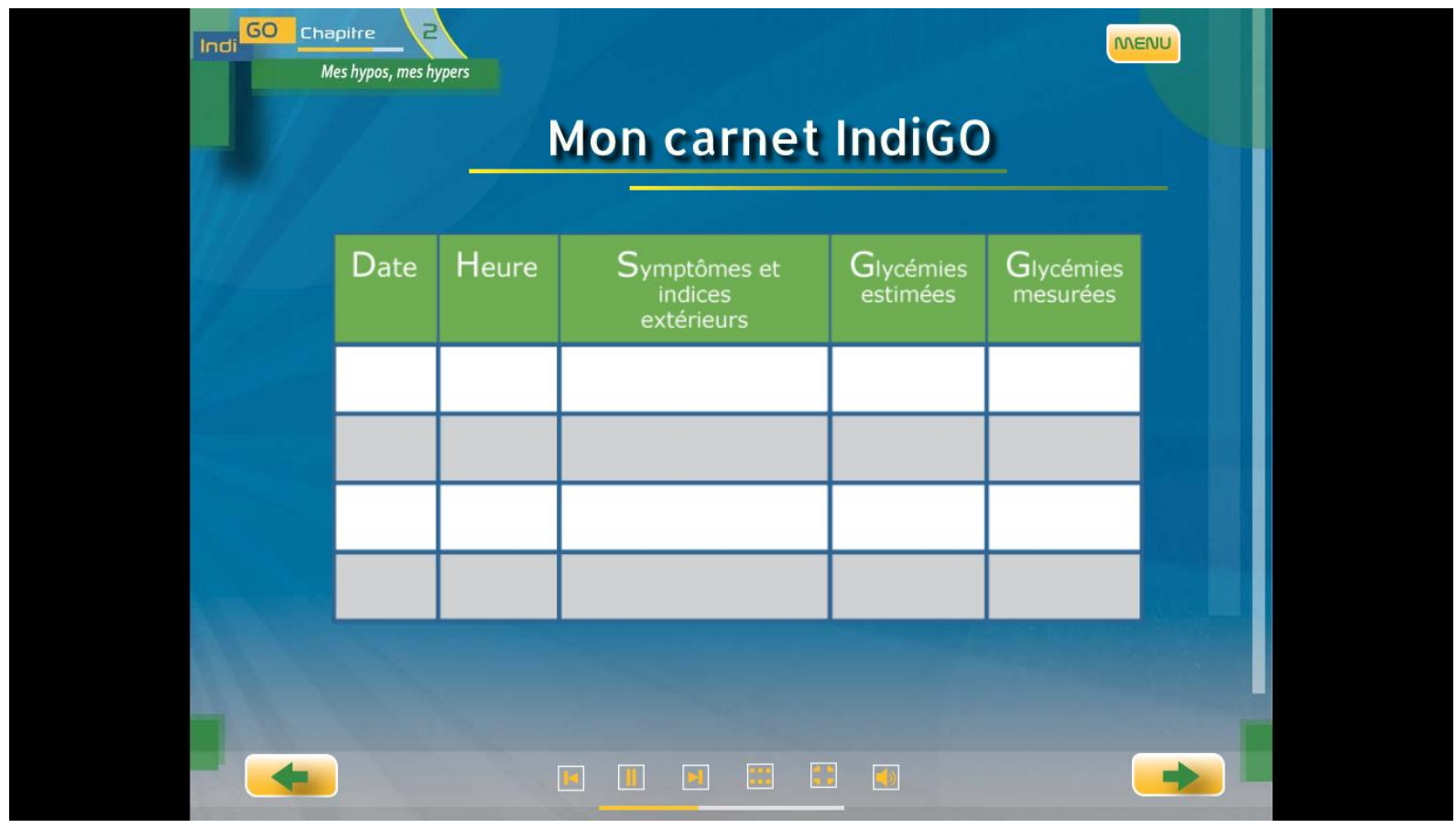


Retranscriptions 
Entretien $\mathbf{n}^{\circ} \mathbf{1}$

Quelle serait la première chose que vous aimeriez me dire concernant le site INDIGO après l'avoir exploré ?

$>$ Je l'ai trouvé très facile d'accès, très agréable à parcourir. C'était très bien expliqué, très... Au début, je me suis dit: "J'ai mon rendez-vous dans pas longtemps, il faudrait que je pense à regarder. » J'ai regardé le premier et puis au final je les ai tous faits dans la foulée.

On va parler de la façon dont vous l'avez visionné. Comment l'avez-vous consulté ? Avez-vous regardé tout en une fois?

$>$ Oui, j'ai commencé par le chapitre 1 et les parties se sont enchainées les unes aux autres et puis je me suis dit autant passer au chapitre 2 et au chapitre 3 .

Vous avez regardé les trois chapitres successivement. Est-ce que vous êtes revenue plusieurs fois sur le site ou est-ce que c'était la seule fois où vous y êtes allée ?

$>$ J'y suis revenue pour voir la $4{ }^{\text {ème }}$ partie du chapitre 3 .

La partie concernant les hyperglycémies et l'acétone ? Et pourquoi avez-vous eu envie de retourner précisément sur ce chapitre-là ?

$>$ Parce que comme je les ai faits tous d'affilée, le dernier j'étais un peu plus fatiguée et du coup je voulais être sûre de ne pas être passée à côté de quelque chose d'important.

Est-ce-que ça vous a paru long ?

$>$ De les faire les trois d'affilée ? Euh... Ils se sont bien enchainés donc du coup j'étais devant mon ordinateur et ça ne m'a pas paru... Je sais que j’y ai passé du temps mais je ne me suis pas ennuyée devant.

Vous étiez seule quand vous l'avez regardé ou avec quelqu'un ?

$>$ Non, j'étais toute seule.

Est-ce que vous avez une idée du temps que vous avez passé dessus ?

$>$ Bien deux heures je pense.

Si vous voulez bien on va parler du $1^{\mathrm{er}}$ chapitre : « Mes objectifs glycémiques ». Vous l'avez donc vu en entier en une seule fois?

$>$ Oui.

Est-ce que vous pouvez me dire en une phrase de quoi traite ce chapitre ?

$>$ De (silence) de, euh... de ce que chacun attend de ses taux et puis de ses prélèvements.

Qu'avez-vous apprécié concernant ce chapitre aussi bien au niveau du contenu que de la forme ?

$>$ Alors moi je n'avais pas spécialement intégré ou compris le fait que chacun devait choisir dans quelle tranche il voulait se mettre. J'avais bien compris les tranches où il ne fallait pas être voilà. Celles où c'était le plus normal d'être et les bornes les mieux et je pensais qu'il fallait à tout prix que je sois dans cette tranche-là. Mais à la suite de ça, je me suis rendu compte que un : ce n'était pas ce qui me convenait et que sans risque pour ma santé, je pouvais décaler cette tranche. Donc du coup, ça a été un petit soulagement. (Rires)

Ça vous a donc permis de vraiment estimer vos propres objectifs glycémiques ?

$>$ Oui.

Et vous avez pu en reparler avec votre diabétologue ?

> Donc j'ai imprimé, j'ai tout imprimé comme ça au moins j'étais sûre. Je lui ai fait part de cette petite observation. Donc il m'a bien expliqué que c'était bon.

Les normes que vous aviez définies grâce au site ?

$>$ Oui. Et du coup, du coup je suis doublement contente de moi sur ce coup-là. (Rires)

Vous m'avez parlé de soulagement ?

$>$ Ah oui parce que ça met une certaine pression de se dire qu'il faut être entre 0,70 et 1,20 avant de manger puis après il ne faut pas dépasser 1,30 après manger. C'est compliqué à gérer en fait. Avec toutes les variables au milieu. Donc du coup, savoir que l'on peut se permettre des écarts, enfin pas des écarts mais une tranche plus large c'est... c'est... ça sérénise un peu.

Y-a-t-il des choses que vous avez trouvées moins bien dans ce chapitre ?

$>$ Non pas... pas des choses qui m’ont marquée en tous cas.

Est-ce que les informations contenues dans ce chapitre ont bien répondu aux questions que vous vous posiez concernant vos objectifs glycémiques ?

$>$ Oui tout à fait, ça a été une belle surprise. (Rires)

Est-ce que vous avez une ou plusieurs suggestions d'amélioration concernant ce chapitre ?

$>$ Non, pas... pas là tout de suite.

On va parler du $2^{\text {ème }}$ chapitre «Mes hypos, mes hypers». Vous l'avez vu et vous l'avez regardé en entier et dans l'ordre?

$>$ Oui.

Est-ce vous pouvez me dire en une phrase de quoi il traite ? 
$>$ Euh... Il me semble que c'est les sensations qu'on éprouve dans chacun des cas. Du coup (silence) ça m'a permis de bien visualiser tout ce qui était sensations pour l'un et pour l'autre et de voir que même si cela se ressemble, pour moi, dans mon ressenti, il y a une grande différence. Bien mettre un mot sur la sensation en fait.

Vous pensez que cela va vous permettre de mieux ressentir les moments où vous faites des hypoglycémies...

$>$... De plus faire attention. Parce que comme je faisais beaucoup d'hypoglycémies, je ne faisais pas forcément attention. Je me disais ça descend, ça varie. Et d'avoir mis un mot, de l'avoir noté : telle sensation il faut faire attention à ça, à ça, à ça... de l'avoir listé en fait, c'est plus clair dans ma tête. C'est écrit quelque part, donc je peux y retourner si j'ai un doute voilà. C'est plus visuel. Et pareil pour les hypers du coup.

Est-ce que vous avez imprimé le carnet INDIGO ? Vous en êtes vous servie ?

$>$ Oui. Je l'ai imprimé et je vais le... je l'ai préparé sur une page Word pour pouvoir le mettre plus accessible en fait.

Vous voulez l'utiliser en version informatique?

$>$ Non, c'est parce que je n'avais pas d'imprimante sous la main mais je vais l'imprimer, comme ça au moins le papier je l'ai toujours sur moi.

Dans ce chapitre, il y a aussi la grille d'analyse des erreurs. Avez-vous aussi commencé la démarche ?

$>$ Oui, c'est très pratique, je l'ai imprimée aussi. J'ai commencé, alors je ne le fait pas à chaque fois, j'attends d'avoir un petit bout de liste et de le marquer. Il m'a fallu un petit moment pour capter toutes les nuances de couleurs et de flèches.

Est-ce que vous pensez que c'est suffisamment bien expliqué ou est-ce que vous pensez que c'est quelque chose que l'on pourrait encore améliorer ?

$>$ Je ne vois pas comment on pourrait l'améliorer donc du coup... Mais en fait il faut se pencher dessus.

C'est donc bien expliqué mais il faut du temps pour l'assimiler ?

$>$ Il faut l'assimiler oui. C'est pas tout le monde qui peut lire un graphique comme ça quoi, un tableau comme ça. Moi ça m'a permis de voir que... au début ça m'inquiétait de voir que le chiffre que j'allais me dire dans ma tête ne correspondait pas à celui que je voyais dans mon lecteur et qu'au final en fait avec cette grille bah j'étais plutôt dans la bonne partie de la grille. J'étais plus dans le vert que dans les autres couleurs. Donc du coup ça rassure aussi.

Y a-t-il des choses dans ce chapitre qui vous ont paru moins bien, toujours au niveau du contenu ou de la forme?

$>$ Non.

Est-ce que les informations contenues dans ce chapitre ont répondu aux questions que vous vous posiez sur les hypos et les hyperglycémies ?

$>$ Tout à fait.

Avez-vous des suggestions d'amélioration concernant ce chapitre ?

$>$ Non plus. (Rires)

On va passer au $3^{\text {ème }}$ chapitre « Mes insulines ». Est-ce que vous avez vu ce chapitre ?

$>$ Oui.

Vous l'avez regardé en entier et dans l'ordre ?

$>$ Tout à fait.

Vous êtes revenue sur la $4{ }^{\text {ème }}$ partie que vous aviez vue mais vous vous étiez posé la question de savoir si vous aviez été suffisamment concentrée. Est-ce qu'au deuxième visionnage vous avez appris des choses ou est-ce que vous aviez finalement tout assimilé en regardant le site en entier en une fois ?

$>$ Non le deuxième visionnage était un peu indispensable parce que je pense que j'étais un petit peu endormie à ce moment-là. En fin de journée c'est un peu plus dur. La seule chose qui me manque un peu dans celui-là, j'ai pas bien cerné les dangers de l'acétone, les risques, les problèmes que ça engendrait.

Vous pensez que l'on vous informe de façon pratique sur la façon de gérer une hyperglycémie mais que l'on ne vous explique pas quels en sont les risques et les conséquences?

$>$ Voilà.

Vous aimeriez pouvoir trouver dans le futur cette information sur le site ?

$>$ Oui. On explique bien que c'est pas bon, qu'il faut l'éviter et comment l'éviter, ce qui est déjà bien, largement bien. On explique bien toutes les procédures.

Vous êtes-vous déjà retrouvée dans cette situation ?

$>$ Oui.

Est-ce que vous avez appris quelque chose ou est-ce que vous le maitrisiez déjà ? 
Alors je ne sais pas si j'ai eu de l'acétone, normalement je pense que oui. Toutes les hypers que j'ai eues, quand le chiffre m'alarmait, tout de suite je me suis tout de suite injecté de l'insuline pour que ça descende. Au pif, j’ai à peu près réussi à le gérer.

Vous ne connaissiez pas le calcul de dose ?

$>$ Non. Voilà, voilà.

C'est quelque chose que vous avez retenu, que vous avez noté ?

$>$ Oui mais je ne l'ai pas noté, ce qui est une erreur, mais je retournerai sur le site le chercher pour l'utiliser et ne pas tomber au pif.

Est-ce que vous avez aussi imprimé les consignes à respecter en cas d'acétone à la bandelette ?

$>$ Non, je n'ai pas imprimé ça encore mais je le ferai prochainement.

Qu'est-ce qui était bien dans ce chapitre ?

$>$ Tous les parallèles qui étaient faits entre les différents traitements, entre les stylos, les pompes.

Vous-même vous avez quel mode d'injection ?

$>$ Moi je suis sous stylo et je réfléchis à la pompe.

Ça vous a donc intéressée d'avoir les deux explications ?

$>$ Savoir que l'on peut passer de l'un à l'autre sans trop de difficultés, temporairement ou pas temporairement. J'ai aussi appris qu'il y avait un troisième type d'insuline que je ne connaissais pas, pour les réglages, etc. Donc c'est toujours sympa d'en découvrir un peu plus.

Et sur les insulines que vous utilisez, avez-vous encore appris des choses ?

$>$ Ça m'a rappelé les temps...

Les délais d'action?

$>$ Les délais d'action. Le délai d'action par exemple de l'insuline lente qui m'a fait tilt en me disant : c'est vrai que si ça varie le soir il y a peut-être ça qui a changé...

De façon pratique, ça peut vous aider dans votre gestion du diabète au quotidien ?

$>$ Oui.

Y a-t-il autre chose que vous avez trouvé bien ?

$>$ Là partie où... tout ce qui était calcul théorique pour les hum...

Les doses de rattrapage?

$>$ Oui les doses de rattrapage, pareil que j'ai pas noté parce que je n'ai pas pris le temps de le faire mais pareil qu'il faut que je le fasse. Jusqu'ici je le faisais un petit peu en me disant : je mets $1,2,4$ ..., mais ça aussi à partir du moment où j'aurai bien intégré le calcul, ça pourra être très pratique.

Ça peut vous rassurer dans votre gestion du diabète au quotidien ?

$>$ Oui.

Est-ce que les informations contenues dans ce chapitre ont répondu à toutes les questions que vous vous posiez sur vos insulines? Vous m'aviez dit non concernant la $4^{\text {ème }}$ partie. Y a-t-il d'autres choses que vous attendiez éventuellement ?

$>$ Non, c'était bien. Complet.

Avez-vous des suggestions d'amélioration concernant ce chapitre ?

$>$ Oui, peut-être plus sur l'acétone.

Donc des explications sur ce qu'est l'acétone, pourquoi ça se produit, en quoi c'est grave, les dangers ?

$>$ Voilà.

De façon générale, concernant tous les chapitres, quel est votre avis concernant la forme du site, son aspect ?

> J'ai trouvé que les différentes parties s'enchainaient bien. Que le côté visuel avec les vidéos, les images, les... que c'était assez pratique. Euh... Par contre toutes les informations ne sont pas écrites, sur certains appareils je n'ai pas forcément le son qui peut sortir, je pensais peut-être à ça qui peut poser problème.

Vous pouvez préciser ? Vous aimeriez que ce qui est dit soit réécris ?

$>$ Il y a certaines parties où la personne, la voix off explique bien tout...

... La voix off ou les témoins?

$>$ Non la voix off. Bah pareil les témoins c'est pas écrit.

Vous pensez qu'il faudrait qu'il y ait à la fois le son et un sous-titre ?

$>$ Oui parce que...

...Vous avez rencontré des problèmes lors du visionnage ?

$>$ Non, mais j'ai un ordinateur chez moi qui est un vieil ordinateur qui n'a pas d'enceintes par exemple et si je l'avais regardé dessus, je serais passée à côté de beaucoup de choses mais euh... Maintenant il y a de plus en plus d'enceintes, mais voilà, c'était le petit détail. (Rires).

D'accord. De façon générale, sur l'ensemble du site, y-a-t-il un chapitre ou un sous-chapitre, qui vous ait semblé moins intéressant ou répondant le moins à des évènements de votre vie quotidienne et si oui lequel ? 
Non, pas en particulier.

Au contraire quel est le chapitre ou le sous-chapitre qui vous a le plus intéressée et qui vous semble le plus utile pour la gestion de votre diabète au quotidien ?

$>$ Moi c'est «Comment je traite mes hypers et mes hypos» qui m'ont beaucoup... j'ai été agréablement surprise.

Est-ce qu'il y a un thème qui vous a manqué, qui n'a pas été abordé dans le site INDIGO et que vous aimeriez trouver dans le futur?

$>$ Pour l'instant vu que les deux derniers chapitres ne sont pas accessibles, pour l'instant à part ces deux-là je ne vois pas spécialement. Après peut-être après les avoir visionnés d'autres impressions, mais pour l'instant je ne vois pas.

Est-ce que vous les attendez ces deux derniers chapitres ? Est-ce que vous voulez être informée lorsqu'ils seront disponibles?

$>$ Oui, si c'est possible.

Quelle est votre satisfaction globale vis-à-vis du site INDIGO ? De 0 à 10.0 : vous n'êtes pas du tout satisfaite et 10 : vous êtes très satisfaite ?

$>10$.

Donc vous estimez qu'INDIGO vous a apporté des éléments qui vont vous aider dans la gestion de votre diabète au quotidien ?

$>$ Tout à fait.

Rappelez-moi lesquels ?

$>$ Tout ce qui était les objectifs à atteindre et ceux possibles et tous les petits réglages, tous les petits détails qui peuvent servir au quotidien dans certaines situations pour gérer la glycémie.

Pour terminer, est-ce que vous conseilleriez ce site à une personne diabétique de type 1 ?

$>$ Oui.

A un membre de votre entourage ?

$>$ Je le montrerai à ma mère par exemple qui panique un peu, enfin, qui n'est pas à l'aise avec le sujet, ça peut la rassurer oui.

Vous en avez déjà parlé ensemble ?

$>$ Je leur ai dit que je l'avais fait parce que du coup c'est eux...

... Quand vous dites eux...

$>$ Mon papa et ma maman. C'est eux qui ont imprimé les documents. Je leur en parlerai, leur donnerai les mots de passe pour pouvoir le faire et puis je leur laisserai le site en accès libre.

Et vous pensez que ça peut les intéresser et leur apporter des informations ? Surtout dans le but de les rassurer parce qu'ils auront plus de connaissances ?

$>$ Oui et comme c'est expliqué très clairement, ça va à l'essentiel. Donc du coup c'est un bon exercice. (Rires)

Est-ce que vous souhaitez me dire autre chose sur le site INDIGO ?

$>$ Il n'est pas accessible sur Google, c'est sur Chrome, donc il a fallu que je télécharge le logiciel.

Ça a été difficile ?

$>$ Non ça s'est bien fait, mais du coup ça m'a surprise.

C'est une suggestion d'amélioration ? Que ce soit accessible sur tous les navigateurs ?

$>$ Oui.

Merci. 


\section{Entretien $\mathbf{n}^{\circ} 2$ :}

Pour commencer, quelle serait la première chose que vous auriez envie de me dire à propos du site INDIGO après l'avoir exploré ?

$>$ En positif ou en négatif?

Ce qui vous vient d'abord.

$>$ Déjà le contenu est vraiment bien, c'est appréciable. Le sommaire enfin le plan, on sait où c'est qu'on va. Moi après ? les petits trucs qui m'avaient dérangé ? c'est plus sur la forme. En fait c'est... par exemple, si on met en plein écran et après on veut faire la flèche « suivant », je crois que ça se... de souvenir ça enlève le mode plein écran et... Il me semble.

Si vous voulez passer à la vidéo suivante ?

$>$ Oui, voilà à la vidéo suivante, je crois en appuyant sur la flèche ça enlève le mode plein écran, il me semble. Je ne suis plus très sûr.

Ce qui vous a gêné c'est que lorsque la séquence s'arrête et que l'on vous demande de cliquer sur la flèche « suivant », cela vous sort du mode plein écran ?

$>$ Oui, exactement. Il me semble.

Et après vous êtes obligé de revenir...

$>$... Après j'avais l'habitude je mettais plus en mode plein écran mais c'est juste ça. C'était sur Firefox aussi. C'est sur Mozilla que je l'ai testé.

Et est-ce que c'était lisible sans mode plein écran ?

$>$ Oui y a pas de soucis, après c'est... suivant la résolution d'écran ça joue pas mal, mais c'était très lisible, pas de soucis là dessus. Ce qu'il y avait après... Moi juste ce qui m'a un peu dérangé aussi... c'est à peu près pareil. Quand on était sur une page, lorsque la vidéo se déroulait où la personne en voix off parlait, donc c'était bien et tout. Après ce qui est parfois dérangeant c'est qu'elle dit une phrase et qu'il faut appuyer sur « suivant », «suivant». Il y a peut-être un ou deux passages qu'il faudrait intégrer dans celui d'avant ou celui d'après pour éviter d'avoir à cliquer sur « suivant » en fait à chaque fois. Après c'est discutable. Après le site était très bien. C'était juste je crois quand le chapitre 2 était fini, on proposait pas de passer directement au chapitre 3. Il fallait retourner dans le menu pour cliquer sur le chapitre 3 . Bon du détail hein.

Vous auriez préféré aller directement au chapitre 3 ?

$>$ Oui que directement après à la fin la flèche dise : voulez-vous maintenant passer au chapitre 3 ? Après ça ne mange pas de pain de cliquer sur menu et de sélectionner le chapitre 3. Parce qu'il y avait ça et le chapitre 3 il était en deux parties aussi, et une fois qu'on a fini la $1^{\text {ère }}$ partie on ne propose pas de passer à la $2^{\text {ème }}$ partie, on repropose de revenir au menu pour cliquer après sur la $2^{\text {ème }}$ partie. Enfin il me semble hein.

Vous vous souvenez entre quel sous-chapitre et quel sous-chapitre ?

$>$ Je sais plus du tout mais il me semblait qu'elle était en deux parties.

Donc entre une des parties du chapitre 3 et l'autre vous avez dû revenir au menu pour recommencer ?

$>$ Oui, voilà. Pour sélectionner l'autre partie.

Et dans les autres chapitres, ça ne vous est pas arrivé ?

$>$ Non les autres chapitres c'était nickel. C'était juste à la fin du chapitre 1, mais ça c'est normal parce qu'il faut se logger et mettre le mot de passe pour le chapitre 2, mais à la fin du chapitre 2 on est obligés de repasser sur le menu pour aller au chapitre 3. C'est juste ça. Après... non après sinon le contenu c'était vraiment bien. Les vidéos c'était sympa. Surtout le chapitre 1, les vidéos j'ai bien aimé.

Les vidéos ? Les témoignages d'autres patients ?

$>$ Oui voilà, les témoignages des gens et puis comme c'était monté, enfin c'était agréable à écouter donc ça c'était sympa. Après bah ce qui m'a fait un peu rire c'était la scène ; je crois que c'était dans le chapitre 2 si je ne me trompe pas ; quand la personne elle s'endort devant la télé et tout, ça ça m'a

Parce que ? fait un peu sourire, c'est normal. (Rire)

$>$ Bah après c'est... non mais c'est juste... après c'est normal c'est pas des acteurs mais c'est un peu sur joué... donc c'est ça qui est marrant mais bon euh...

Est-ce que vous avez appris quelque chose de cette scène ?

$>\mathrm{Si}$, si. Il y avait... Bah du coup sur le Glucagon, ils rappelaient un peu comment ça se préparait, comment se l'injecter et tout donc euh... C'est pas vraiment que j'ai appris, mais des rappels ça fait pas de mal donc euh... Ça c'était intéressant. Et après bah si moi j'ai bien aimé le $1^{\text {er }}$ chapitre quand les deux témoins ; alors je ne me rappelle plus du nom des personnes; mais ils parlaient... Par exemple lui il faisait du sport ou autre et l'autre personne je sais plus ce qu'elle faisait. C'était une dame, je ne me souviens plus. Le chapitre 1 je l'ai trouvé vraiment intéressant. Après je crois c'était 
le chapitre 2 où il y avait la droite où il fallait situer par rapport à où on ressentait notre glycémie. J'ai trouvé ça intéressant parce que ça je ne l'avais jamais vu.

Qu'est-ce qui vous a plu dans les témoignages ?

$>$ En fait bah... je trouvais bah déjà que c'est pas mal d'avoir des avis de diabétiques. Enfin je ne sais pas si c'étaient des vraies personnes diabétiques.

Vous avez un doute sur le fait que ce soient des vraies personnes diabétiques ?

$>$ Non, mais c'était juste pas signalé donc je pense pas hein. Enfin pour moi c'étaient des vrais diabétiques. C'était juste, enfin c'était pas signalé je crois, quand il y a en-dessous la légende avec son nom, je sais pas s'il y avait écrit diabétique. Donc c'était peut-être intéressant de savoir depuis quand il est diabétique en fait, c'est quand même assez sympa.

Vous pensez qu'il vous manquait des informations sur les témoins?

$>$ Oui voilà. Sur les témoins de savoir si c'étaient des vrais diabétiques, à quel stade ils en étaient. Enfin ça faisait combien de temps qu'ils avaient leur diabète, si c'était récent ou plutôt ancien. Ça ça aurait été pas mal.

Et ça vous a empêché de vous identifier à eux ?

$>$ Non, non, pas du tout, c'était très pertinent ce qu'ils disaient. Non. C'était juste au début on se pose quand même la question dès qu'on voit la personne, est-ce que... parce que voilà après on sait pas si c'est une vraie personne ou pas, parce que ça pourrait être quelqu'un qui lit un texte.

Et par rapport au sur jeu entre guillemets que vous avez ressenti concernant la vidéo sur les hypoglycémies sévères, alors effectivement c'est une séquence où il y a une mise en scène. Sur les autres témoignages avez-vous eu des ressentis similaires ?

$>$ Non les autres... Non, c'est très clair, très clair. On s'identifie très facilement et tout. Non c'était très bien. Après je ne me souviens plus le nombre de personnes qu'il y avait. Bah y en a deux dans le chapitre 1, bah le couple assez âgé dans le chapitre 2 et dans le chapitre 3 je ne me souviens plus trop, je sais plus trop. Après parmi les figurants je me rappelle plus vraiment, je sais qu'il y en a mais je sais plus. Non mais c'était bien clair. Après ah oui, là je m'écarte un peu du sujet, mais c'était au début, en fait moi j'ai l'habitude de pas mettre de son sur mon ordi et il faudrait peut-être au lancement du site, au menu, dire que le support fonctionne avec de l'audio. Parce que du coup au début je suis arrivé sur le site et en fait il y avait la dame qui parlait en voix-off et du coup moi je savais... je comprenais pas ce qui se passait parce que j'avais pas le son activé. Après je me suis rendu compte qu'il y avait besoin d'activer l'audio en fait. Donc oui, c'est peut-être un petit truc à mettre sur le côté.

Spontanément, avez-vous d'autres choses à ajouter ?

$>$ Non, là pour le moment, c'est bon.

J'aimerais maintenant que vous me racontiez comment vous avez visionné le site ?

$>$ Oui. Et ben du coup je l'ai visionné en deux parties. Donc j'ai fait d'abord le chapitre 1 un soir ; donc la présentation et le chapitre 1 ; et après... donc ça c'était le jeudi soir si je ne me trompe pas. Et le samedi matin je me suis fait le chapitre 2 et le chapitre 3.

C'est par manque de temps que vous avez arrêté ou parce que vous n'aviez plus envie, vous étiez moins concentré ?

$>$ Bah non c'est... au bout d'un moment ça fait beaucoup d'informations quand même, enfin vu qu'il y a quand même quatre heures de vidéos je crois, enfin quelque chose comme ça. Du coup c'est pas mal de faire une petite pause et de le voir étape par étape. Voilà. Après je l'ai regardé aussi avec ma copine donc c'était agréable pour elle vu qu'elle est moins au courant que moi de ma maladie. Pour elle c'était pas mal de voir... bah de voir ce que disait le site parce que moi je suis quand même un peu plus au courant qu'elle donc pour elle c'était plus agréable euh...

... Elle a vu les trois chapitres ?

$>$ Non, elle a vu que le chapitre 2 et 3 .

Avez-vous pu en rediscuter ensuite avec elle ? Qu'est-ce qu'elle en a pensé ?

$>$ Pour elle c'était intéressant parce que du coup bah pareil le Glucagon... le kit elle savait pas trop comment fonctionnait donc ça c'était pas mal. Puis après même en règle générale, c'était pas mal de discuter autour... Bah elle aussi elle a dit que c'est pas mal aussi le système... la droite là que... pour savoir par rapport à la glycémie si on se situe, au-dessus ou en dessous, ça aussi on en a un peu parlé et tout. Ça et puis, oui, elle elle a appris oui beaucoup, moi vu que j'étais quand même au courant donc c'était un rappel ça fait pas de mal, elle elle a appris quand même pas mal de choses.

Et est-ce que c'est quelque chose qu'elle attendait, avoir des informations sur le diabète ?

$>$ Je sais pas, mais avec Internet, c'est un peu dur de savoir ce qui est juste, ce qui est faux. Là au moins vu que déjà... enfin on sait que ça a été recommandé par mon diabétologue.

Elle était déjà allée sur Internet chercher des informations ? 
$>$ Bah elle avait regardé oui mais après il n'y a pas tout le schéma explicatif enfin là c'est bien détaillé, les objectifs, quelle glycémie il faut avoir avant, après les repas, enfin au moins on a des sources sûres parce que après on peut avoir de tout et n'importe quoi. Donc ça c'était agréable.

Est-ce que c'est vous qui lui avez demandé de regarder ou est-ce que c'est elle qui vous l'a proposé ?

$>$ Bah un peu les deux. En fait dès le début j'ai... donc j'ai regardé le chapitre 1 tout seul et du coup là j'avais vu mon Professeur et tout donc après on en a parlé, je lui ai dit : « Ah tiens, j'ai vu une vidéo, y a mon Professeur qui parlait ».

Votre Professeur ?

$>$ Le Professeur BENHAMOU, mon diabétologue. Et du coup après bah quand elle est venue on s'est proposé en quelque sorte de regarder les deux chapitres suivants.

Elle vous a dit que ça l'avait intéressée, qu'elle avait appris des choses ?

$>$ Oui, bah elle oui, elle a beaucoup plus appris que moi vu que je connaissais quand même une bonne partie. Mais si, si c'est agréable. Enfin les chapitres s'enchainent bien, juste c'est de cliquer comme j'ai dit donc sur la flèche parfois un peu trop souvent peut-être, c'est peut-être mieux de laisser défiler...

Vous dites qu'il y a beaucoup de choses que vous connaissiez. Vous avez quand même le sentiment d'avoir appris des choses ?

$>$ Euh bah oui.

Vous sauriez me dire quoi ?

$>$ Après en particulier... Je sais pas si on peut dire vraiment appris parce que je connaissais déjà par exemple les intervalles de glycémie dans lesquelles il faut être mais euh... Les normes en général, on va dire. Et donc euh...

... Est-ce que ça vous a permis de peaufiner votre objectif ?

$>$ Oui, à peu près, en quelque sorte. Ce qui était pas mal, ouais voilà, ce qui était intéressant c'était dans le chapitre 1 justement, il fallait dire combien de fois on avait été en hypoglycémie sur les 50 derniers résultats. Sur les 50 d'avant aussi combien on avait été en hyperglycémie. Donc ça c'était pas mal, ça permet de faire un petit point.

Ça vous l'avez rempli ?

$>$ Oui voilà, ça je l'ai rempli. Et ça m'a montré que j'étais un peu plus souvent en hypoglycémie qu'en hyper, mais ça j'étais au courant. Mais c'est pas mal, ça permet de prendre un peu du recul quand même. Là il faut dire aussi avec mon appareil de glycémie j'ai directement les courbes, les camemberts et tout ça donc il me signale également sur les sept derniers jours combien de fois j'ai été en hypoglycémie donc euh...

D'accord. Est-ce que vous pensez que le site va provoquer une discussion avec votre généraliste ou votre diabétologue sur ce point-là ?

$>$ Peut-être revoir avec lui dans mon cas peut-être les intervalles de glycémie qu'il faut avoir parce que je fais pas mal de sport aussi donc ça sera à rediscuter avec lui, ça sera une bonne occasion.

Il y a d'autres choses que vous avez apprises?

$>$ Le coup de la droite comme j'en ai parlé, ça j'étais pas au courant donc j'ai appris ça, c'est pas mal.

Expliquez-moi ça.

$>$ En fait si je ne me trompe pas, c'est une diagonale. En gros c'est avant de faire la glycémie on...

... Ah c'est la grille d'analyse des erreurs ?

$>$ C'est ça, c'est en fait avant de faire la glycémie, on doit essayer de ressentir à combien on est approximativement puis après on regarde si par rapport à la vraie valeur si on était complètement à l'ouest ou si on était plutôt juste.

D'accord. C'est un outil qui vous intéresse ? Vous pensez l'imprimer ?

$>$ Oui, ça je veux bien l'imprimer après j'avais pas d'imprimante à disposition, c'est pour ça que je l'ai pas imprimé. Après pas le faire tous les jours mais le faire pendant deux semaines pour voir à peu près, parce que ça permet de faire un point, c'est pas mal. Après c'est pas mal pour prendre du recul un peu, enfin on va dire faire le point sur ça. Du coup c'est pas mal pour se consacrer on va dire un peu de temps à soi-même parce qu'avant de faire la glycémie on n'estime pas vraiment à combien on va être. Donc là ça permet au moins de se poser, peut-être se concentrer trente secondes pour dire : «Tiens, comment je me sens ? Est-ce que je me sens plutôt bien ou plutôt mal ? ». Comme ça, ça permettrait peut-être je pense de mieux ressentir les hypoglycémies ou autres.

Est-ce que vous envisagez de l'utiliser en association avec le carnet INDIGO ?

$>$ Du coup le carnet INDIGO je ne m'en sers pas vraiment vu que sur mon appareil j'ai déjà toutes les valeurs et tout et puis après avec le logiciel sur mon ordinateur, quand je le branche j'ai toutes les courbes et tout donc euh.

D'accord. Le carnet INDIGO permet aussi de réfléchir aux conditions : ce que vous avez mangé, ce que vous avez fait... 
$>$... C'est vrai, il n'y a pas ça sur le lecteur. Après ça peut être pas mal pour une personne qui a un diabète qui n'est pas très bien équilibré, ça peut-être pas mal d'avoir ça pour faire un point, bien comprendre, pour expliquer les glycémies élevées ou basses, parfois c'est un peu dur de l'expliquer donc euh, avoir cet outil-là dans ce genre de situation ça peut être bénéfique je pense.

On va revenir sur la façon dont vous avez visionné le site. Vous m'avez dit que vous l'aviez visionné une partie tout seul et une partie à deux. Vous l'avez visionné dans l'ordre des chapitres ?

$>$ Oui, c'est ça.

Et à l'intérieur des chapitres, vous l'avez visionné dans l'ordre des sous-chapitres?

$>$ Oui, à peu près. Il y a juste peut-être un chapitre que j'ai un peu passé, c'est le chapitre «Comment savoir où j'en suis au niveau de ma glycémie ?» il me semble, je ne sais plus si c'est celui-là. En tout cas il y a un des sous-chapitres que j'ai sauté parce que j'étais déjà au courant de la situation.

Il y en a un dès le titre vous vous êtes dit : « Je connais, je n'en ai pas besoin »?

$>$ Exactement parce qu'en fait quand on lance un chapitre on nous propose donc de choisir les souschapitres et du coup il y en avait un... il me semblait bah du coup que ça faisait un peu redondant avec le chapitre précédent.

D’accord, dans un chapitre vous en avez sauté un, sinon vous avez fait dans l'ordre proposé ?

$>$ Oui après j'ai tout fait dans l'ordre.

Est-ce qu'il y a des chapitres sur lesquels vous êtes revenu ?

$>$ Non j'ai tout visionné comme ça une fois. Après peut-être dans le futur, je pense qu'il y a 2 ou 3

Lesquels ? chapitres pour lesquels j'aurai besoin de revenir.

$>$ Celui qui m'intéresserait peut-être le plus pour le moment ce serait celui avec la grille d'analyse des erreurs. Peut-être revisionner celui-là.

Est-ce que vous l'avez compris facilement ? Est-ce que vous pensez que c'est bien expliqué ?

$>\mathrm{Si}$, oui. Après je suis en école d'ingénieur donc la forme c'était bien compréhensible mais ma copine aussi. Après c'est pour les personnes qui ont un peu plus de mal... mais pour moi ça ne posait aucune difficulté de ce côté-là.

Le fait de revoir certains chapitres, c'est parce que vous pensez que vous aurez oublié ou pour réapprofondir?

$>$ Oui bah pour ré-approfondir, refaire un petit rappel et puis vu que c'est bien structuré, on peut directement aller... on n'est pas obligé de se coltiner tous les chapitres pour aller à celui qui nous intéresse. Donc au final si on a un petit doute à un endroit, on peut facilement y accéder à nouveau.

Très bien. On va parler du $1^{\mathrm{er}}$ chapitre « Mes objectifs glycémiques ». Est-ce qu'en une phrase vous pourriez me dire de quoi il traite ?

$>$ Du coup pour moi c'était plus... De ce que j'ai retenu c'était plus en fait sur la découverte un peu en gros du diabète, comment les personnes géraient leurs hypoglycémies, leurs hyperglycémies en général en fait. Toutes les actions qui entrainaient... enfin comment influait le diabète chez ces personnes-là en fait. Ce que je retiens beaucoup bah c'est les deux entretiens vidéo avec la personne qui faisait du sport, qui disait qu'il faisait du basket et l'autre dame aussi.

Vous vous êtes identifié à ces témoins ?

$>$ Oui, oui. Après ce qu'ils disaient c'était juste. Enfin c'était pertinent.

Ça vous a apporté des choses ou ça vous a réconforté dans...

$>\quad$... Oui voilà réconforté, exactement. C'était le mot que je cherchais, oui.

D'accord. Ce que vous avez apprécié c'est le fait qu'il y ait des témoins.

$>$ Oui, c'est agréable d'avoir l'avis de personnes diabétiques. Le seul truc... le seul point intéressant ce serait de mettre... de dire depuis quand il a le diabète pour voir à peu près si c'est un nouveau ou s'il a de l'expérience, à quel stade il en est. Enfin pour moi c'est intéressant.

Est-ce qu'il y a d'autres choses qui vous ont plu dans ce chapitre ?

$>$ Euh bah après... (Silence) Le fond pour moi, le contenu était bien et tout, enfin c'était bien expliqué, clairement, étape par étape, enfin ça allait pas trop vite sur le dosage d'informations. D'ailleurs c'est dans ce chapitre-là où ils expliquent; après vu que j'ai fait le stage je l'ai déjà vu ; le système du corps humain avec comment fonctionne le sucre dans le corps, c'était dans ce chapitrelà.

Vous l'aviez déjà vu en stage d'éducation thérapeutique?

$>$ Oui, voilà.

Est-ce qu'il y a des choses qui étaient moins bien dans ce chapitre au niveau du contenu ou de la forme?

$>$ Non

Est-ce les informations contenues dans ce chapitre ont répondu aux questions que vous vous posiez sur vos objectifs glycémiques? 
(Silence) Disons oui. Au moins ça m'a permis de certifier la plage de glycémie qu'il fallait avoir.

Donc ça vous a conforté ?

$>$ Oui ça m'a conforté oui.

Est-ce que vous avez des suggestions d'amélioration concernant ce chapitre ?

$>$ Sur ce chapitre-là, euh... pas spécialement. Après, c'était peut-être... J'ai pas essayé de faire bugger le site ou autre mais quand il faut remplir des informations parfois à la main... Après je sais pas si une personne a une lettre ou quelque chose comme ça, si ça fait crasher le site ou peut-être mettre un menu déroulant, enfin ça je...

En tout cas vous vous en êtes servi et vous n'avez pas eu de difficultés...

$>$... Je l'ai bien fait, je l'ai fait normalement et je n'ai pas eu de...

... Et vos symptômes vous les avez notés ?

$>$ J'ai coché en fait sur le site donc ça c'était agréable de cocher.

On va passer au chapitre 2 « Mes hypers, mes hypos ». Donc vous l'avez regardé avec votre amie. Vous avez sauté un sous-chapitre parce que d'après le titre c'est des choses que vous connaissiez. Sinon vous avez regardé le chapitre dans l'ordre.

$>$ Oui, c'est ça.

Est-ce que vous pouvez me dire en une phrase de quoi traite ce chapitre ?

> Pour moi c'était surtout les hypoglycémies donc avec la scène là, le couple qui est devant la télé, avec donc l'injection du Glucagon, donc l'infirmière aussi qui explique comment ça marche. Donc c'est surtout ça que je retiens de cette partie-là. Après j'ai pas le souvenir qu'il traite beaucoup des hypers. Peut-être j'aurais préféré, en amélioration, enfin qu'il parle aussi un peu plus des hyperglycémies.

Vous n'avez pas trouvé de réponses dans le site à quoi faire quand on est en hyperglycémie ?

$>$ Moi, je sais quoi faire mais après pour une personne qui n'est pas au courant. De mémoire comme ça, ils insistaient beaucoup donc sur les hypoglycémies avec l'injection du Glucagon mais de mémoire comme ça enfin, les hypers ça m'a un peu moins marqué donc euh. Après je sais pas si vraiment ça y était ou pas hein.

Vous auriez aimé qu'on vous explique plus...

$>$... Enfin pas plus mais pareil, en cas d'hyperglycémie ce qu'il faut faire. Donc euh on se mesure la glycémie, bien être sûr que c'est au moins $2 \mathrm{~h} 1 / 2-3 \mathrm{~h}$ après l'injection d'insuline pour pas fausser les résultats...

D'accord. Qu'est-ce qui vous a plu dans ce chapitre ?

$>$ Après c'était bien d'avoir un retour sur l'infirmière, comment injecter le Glucagon, ça c'était intéressant. Après il était bien, c'était la mise en scène qui m'a fait un peu rire comme je vous ai dit, après c'est normal.

Comment vous pensez que l'on pourrait l'améliorer ?

$>$ De mémoire c'était la personne elle s'est endormie devant la télé donc son amie arrivait, elle essayait de le réveiller «Ah non tu dors pas». Donc après de mémoire elle fait: «Ah oui, l'infirmière m'a dit de faire ça ». Donc là on a un petit flash back un peu sur ce qu'a dit l'infirmière et après du coup elle fait en temps réel. Non après c'était pas mal. Après peut-être que c'était un peu trop accentué sur le fait, il est endormi devant la télé et puis après elle le secoue machin. Ça prenait peut-être un peu de temps je veux dire par rapport au but que ça visait au final. C'était peut-être disproportionné en fait le temps de découverte qu'il est en hypoglycémie. Après le temps de qu'estce qu'il faut faire et tout ça prenait le bon temps mais peut-être ça prenait un peu trop de temps la découverte de l'hypoglycémie on va dire. Bon après c'est relatif hein.

D'accord. Est-ce que les informations contenues dans ce chapitre ont répondu aux questions que vous vous posiez sur les hypos et les hypers?

$>$ Oui bah surtout sur les hypos, voilà.

Est-ce que vous avez d'autres suggestions d'amélioration ?

$>$ Non, pas particulièrement.

On va passer au $3^{\text {ème }}$ chapitre : «Mes insulines ». Vous étiez avec votre amie. Vous l'avez vu en entier et dans l'ordre?

$>$ Non, j'ai regardé que les trois premiers sous-chapitres en fait.

D'accord. Est-ce que vous pouvez me dire en une phrase de quoi traite ce chapitre ?

> Pour moi c'était surtout sur les sites d'injection de l'insuline en fait. Il y avait 2-3 images. Ce que je retiens surtout c'était sur l'injection sur le bras. Je crois que c'était Eléonore qui faisait ça. C'était intéressant de voir les différentes possibilités de sites d'injections ça c'est pas mal à parler dessus. Après je sais pas si dans ce chapitre-là il parlait des lipostrophies c'est ça ?

Lipodystrophies. Oui.

$>$ Lipodystrophies. Je sais pas s'ils en parlaient dans ce chapitre-là. 
Si, ils en parlaient un petit peu.

$>$ Pour moi ce qui aurait été bien c'était de voir une image à quoi ça ressemble une lipodystrophie enfin pour montrer la différence entre avant ou après ou montrer une personne qui n'en a pas, une personne qui en a. Parce que moi du coup je suis au courant dans le cadre de ma maladie mais pour mon amie par exemple qui ne connait pas, pour elle ça aurait été pas mal d'avoir un exemple de ce que c'est.

Y-a-t-il d'autres choses qui vous ont plu ?

$>$ Bah pareil les témoignages donc de personnes qui sont diabétiques c'est agréable à entendre. Puis ce qu'ils disent c'est pertinent aussi, donc ça c'est intéressant. Après globalement aussi c'est pas mal, je trouve ça bien la voix off qui parle, ça lit pas forcément les phrases qui sont affichées, mais ça dit des informations en plus donc ça c'est agréable. Oui le seul point donc c'était ; je sais plus si c'était dans ce chapitre-là ou le chapitre 2 ; où il fallait du coup peut-être trop souvent appuyer sur «suivant » alors que parfois vraiment elle parlait dix secondes et après on nous proposait d'appuyer sur « suivant».

Et ça vous pensez que ça déconcentre?

$>$ Bah oui du coup ça casse un peu le rythme.

Est-ce que ça vous a lassé ?

$>$ Non, non parce qu'au final ce qui est pas mal c'est de voir la part de progression, où peut voir aussi où on en est par rapport au chapitre, si ça finit bientôt où pas, ça aussi c'est agréable.

Donc vous pensez qu'une idée d'amélioration, ça serait de moins découper les sous-chapitres ?

$>$ Bah peut-être oui. Bon après c'est pour la personne qui veut directement aller dans le chapitre 2 à tel endroit ça peut être pas mal. A l'intérieur du sous-chapitre, c'est quand même pas mal d'avoir étape par étape mais c'est vraiment parfois elle parlait... ouais cinq à dix secondes et après on proposait de cliquer sur suivant donc là ça aurait été utile de l'intégrer directement, après c'est pas tout faire le chapitre d'un bloc mais mieux gérer en fait les temps... tous les combien on doit appuyer sur suivant en fait.

D'accord. Si on revient au chapitre 3 en particulier, qu'est-ce qui était moins bien ?

$>$ Moins bien ? Pour moi rien.

Est-ce que les informations contenues dans le chapitre ont répondu aux questions que vous vous posiez sur vos insulines?

$>$ Pour moi c'était bien clair. Il y a juste le coup des lipodystrophies où j'aurais aimé voir des photos ou un exemple, qu'ils en traitent un peu plus, que ça frappe un peu plus en fait.

Est-ce que vous avez d'autres suggestions d'amélioration ?

$>$ Non.

De façon générale, si on reprend tout le site, quel est le chapitre ou le sous-chapitre qui vous a semblé le moins intéressant ou répondant le moins à des évènements de votre vie quotidienne ?

$>$ Pour moi c'était la scène donc de ménage un peu en quelque sorte avec l'hypoglycémie. C'était trop accentué sur le début de cette scène-là avant que ça commence réellement et qu'on voit comment faire le Glucagon. Peut-être ce passage-là.

Quel est au contraire le chapitre ou le sous-chapitre qui vous a le plus intéressé ou qui vous semble le plus utile pour gérer votre diabète au quotidien ?

$>\quad$ Moi j'ai bien aimé le $1^{\text {er }}$ chapitre avec l'enchainement des témoignages qui sont bien structurés, qui passent étape par étape et puis confronter les deux points de vue entre un jeune en quelque sorte et une personne un peu plus adulte. Moi j'ai bien apprécié ce chapitre-là.

Quel est le thème que vous n'avez pas trouvé dans le site INDIGO qui vous a manqué et que vous aimeriez éventuellement trouver dans le futur?

> Bah après vu qu'il n'était pas complet, c'est sûr que prendre en compte l'alcool quand on est jeune, étudiant et tout, donc avoir un chapitre sur l'alcool, ça peut être vraiment intéressant.

Donc qui sera donc dans le chapitre « Mon alimentation ».

$>$ Et aussi les activités physiques.

Est-ce que vous souhaitez être informé quand ces chapitres seront disponibles ?

$>$ Oui.

Et en dehors de ces deux chapitres avez-vous d'autres idées de thèmes ?

$>$ Bah après comme ça... L'alcool et les activités physiques ça me semble essentiel. Après non.

Quelle est votre satisfaction globale vis-à-vis du site INDIGO de 0 à 10,0 : vous n'êtes pas du tout satisfait et 10 : vous êtes très satisfait ?

$>$ Je mettrais 8. Après moi c'est juste sur la forme donc les couleurs elles sont peut-être un peu flashy, enfin le jaune des flèches suivant en contraste avec le bleu clair derrière ça fait peut-être un peu trop flashy, mais après euh...

D'accord. Vous auriez préféré comment ? 
$>$ Bah non jaune comme ça très bien mais peut-être juste la couleur, diminuer l'accentuation enfin je sais pas trop après c'est de la mise en forme hein.

C'est ce qui aurait manqué pour que vous mettiez 9 par exemple ? Autre chose ?

$>$ Après bah du coup comme j'ai dit un peu les liens entre les chapitres, enfin directement proposer le chapitre suivant. Mais après sinon la vidéo, enfin les images et tout à l'écran c'était clair, ça prenait une bonne place par rapport à ce qu'on voyait. Le montage, la voix off c'est agréable. Et c'est juste au début du site de dire attention ça fonctionne avec l'audio et tout. Non après globalement, le site était bien.

Est-ce que vous estimez qu'INDIGO vous a apporté des éléments qui allaient vous aider dans la gestion de votre diabète au quotidien ?

$>$ Euh... (Silence) Oui, déjà la grille d'erreur. Ça et puis ça permet donc de rappeler les bases... les bases d'être diabétique, enfin toutes les problématiques autour. On apprend quand même pas mal de choses. Après vu que moi j'étais au courant, pour moi c'était pas nouveau on va dire en quelque sorte. Pour ma copine elle qui était un peu moins au courant, pour elle c'était bien de...

Vous avez déjà eu des séances d'éducation thérapeutique ? Vous en avez eu combien ?

$>$ J'ai déjà fait 3 jours de séances en stage ici au CHU. Et aujourd'hui, je refais une journée d'évaluation de stage.

Et vous pensez qu'il faudrait proposer le site plutôt à des gens qui ne sont jamais allés en séance d'éducation thérapeutique ou vous pensez que c'est intéressant de le présenter aussi à des gens qui....

$>\quad \ldots$ Si ça fait pas de mal, après c'est peut-être un peu répétitif mais je pense pour les compagnes ou les personnes qui accompagnent une personne qui est diabétique, c'est quand même pas mal, ça permet de mieux comprendre la maladie. Ça permet aussi de prendre du recul, de prendre du temps pour soi et s'écouter.

Vous pensez que c'est intéressant pour l'entourage. Donc justement, vous l'avez montré à votre amie. Est-ce que vous conseilleriez le site à d'autres personnes de votre entourage ?

$>$ Oui, à mes parents enfin ou à la famille, à toutes les personnes assez proches.

Vous leur en avez parlé ou vous compter le faire ?

$>$ Pas encore, j'en ai juste parlé à mes parents mais je leur ai pas encore donné le lien. Je pense que ça peut être pas mal pour comprendre la maladie.

Vous pensez qu'ils vont regarder, qu'ils seront intéressés ?

$>$ Bah je pense, ouais. Après, vu qu'il y a des questions c'est propre à la personne qui est diabétique, par exemple : «Quelles sont vos glycémies ?», ce genre de chose, peut-être qu'ils sauteront ces chapitres-là. Mais juste comprendre en général le mécanisme et tout, pour les personnes qui n'ont aucune connaissance c'est intéressant.

Est-ce que vous conseilleriez le site INDIGO à une autre personne diabétique de type 1 ?

$>$ Oui. Après j'en connais pas, donc euh.

Est-ce que vous avez autre chose à me dire ?

$>$ Non, je pense qu'on a fait un bon tour.

Une dernière chose, dans le questionnaire, à la question «Vous êtes vous senti déstabilisé dans la gestion de votre diabète au quotidien ? » vous avez répondu «Oui, plutôt d'accord ». Est-ce que vous pouvez aller plus loin ?

$>$ (Silence) Je sais pas trop pourquoi j'ai coché ça, mais c'est un peu entre les deux. Peut-être avec les seuils de tolérance qu'ils fixent en fait dans les glycémies, peut-être qu'il y a des personnes ça va être un peu trop fixe ou ils vont vraiment s'inquiéter, je sais pas.

Et vous-même, vous vous êtes senti déstabilisé ?

$>$ Bah, peut-être un peu. Quand par exemple après le repas j'ai une glycémie à 0.8 ou 0.9 , sur le site ils conseillent vraiment à partir de 1.2, enfin je sais plus les valeurs, mais du coup ça m'avait un peu déstabilisé.

Est-ce que vous allez rediscuter de ça avec le Pr BENHAMOU ?

Merci.

Oui, ça fera l'occasion je pense. 


\section{Entretien $\mathbf{n}^{\circ} 3$}

Pour commencer, quelle serait la première chose que vous auriez envie de me dire après avoir exploré le site INDIGO ?

$>$ Je l'ai trouvé euh... plutôt bien fait, euh... ça c'est plus au niveau forme.

Oui. Qu'est-ce-qui va a plu en particulier dans la forme ?

$>$ Ce qui m'a plu c'est que déjà c'était facile d'avoir accès aux différents menus, aux différents chapitres plutôt, euh..

Donc la navigation à l'intérieur du site ?

$>$ Voilà, ça c'était pas mal. Et les couleurs aussi, c'était assez attrayant. Les vidéos, les images, les graphiques que l'on voit euh... se faire.

Toujours sur le plan de la forme ? Le fait que les graphiques apparaissent progressivement ?

$>$ Voilà c'est ça. Oui, oui ça c'était pas mal.

C'est une forme qui vous plait. Est-ce que ça aide aussi à la compréhension selon vous ?

$>$ Ah oui, oui tout à fait. Oui, si, si ça aide à la compréhension, le fait justement que ce soit fait étape par étape et qu'on voit... par exemple il y avait un... un diagramme euh...

La grille d'analyse des erreurs ?

$>$ Voilà, c'est ça. Et donc ça c'était bien, parce que ça partait... on voyait vraiment les deux premières zones, la construction étape par étape. Ça c'était bien.

Ça suivait les étapes du raisonnement et du coup c'était simple à comprendre ?

$>$ Voilà, tout à fait. Oui, oui, oui. Après euh au niveau du fond, c'était intéressant oui par rapport aux vidéos, parce que forcément, c'est plus parlant d'avoir affaire directement à des...

...Les témoignages ?

$>$ Oui à des témoignages. Oui forcément on est tout de suite dans le concret donc ça parle tout de suite.

Est-ce que vous avez trouvé que les exemples étaient proches de ce que vous vivez au quotidien ?

$>$ (Silence) Proches euh... Par rapport à ma situation actuelle non, je ne dirais pas que c'était... je ne dirais pas que c'était proche.

Alors qu'est-ce qu'il manquait pour s'en approcher? Y-a-t-il quelque chose que vous n'avez pas trouvé et que vous auriez aimé trouver ? Comment aurait-on pu l'améliorer ?

$>$ Déjà, il y avait pas mal de gens qui pratiquent une activité sportive.

Ce qui n'est pas votre cas?

$>$ Ce qui n'est pas mon cas. Et puis il y avait aussi... les gens parlaient pas mal de leurs hypoglycémies. Moi je... Bah disons que pour ma part, mes glycémies je les fais juste avant de manger et je ne suis pas en hypo. En plus de ça, mes hypos je ne les ressens pas. Enfin, je les ressens vraiment quand je suis à $0,4-0,3$.

Et est-ce que justement la grille d'analyse des erreurs, le carnet INDIGO pourraient vous correspondre?

$>$ Ça j'ai trouvé que c'était pas mal effectivement. Ça j'aime bien parce que c'est...

... Et vous pensez l'utiliser ?

$>$ Oui, je pense l'utiliser.

Vous l'avez imprimé ?

$>$ Je l'ai imprimé. Alors je ne me suis pas encore plongé dedans. (Rires) J'ai fait un peu comme la personne qu'on voit dans une des vidéos, qui jette son carnet. (Rires) J'ai pas fait ça, mais c'est à peu près l'idée. Et donc ça je pense que c'est très très bien.

Vous me dites que c'est très très bien. Vous pouvez aller plus loin ?

$>$ Et bien c'est très bien parce que effectivement ça permet de faire la corrélation entre vraiment ce qui est mesuré donc ce qui est objectif et son ressenti. Donc du coup ça permet vraiment de voir si on est complètement à côté de la plaque ou si on est vraiment euh... proche euh... de la cible, enfin de ce qui est mesuré. Donc ça j'ai trouvé que c'était bien.

Et vous votre objectif pour l'utiliser, le but, ce serait de mieux ressentir vos hypos parce que vous ne les ressentez pas?

D'accord.

Oui, c'est ça. Enfin, d'après moi je n'en fais pas.

$>$ J'en ai eu deux. Une la nuit. Donc celle-là, je l'ai ressentie tout de suite parce que je me suis réveillé et j'avais la tête un peu qui tournait, celle-là je l'ai ressentie tout de suite. Et une fois en marchant aussi.

D'accord. Mais vous m'avez dit que vous ne pensiez pas en faire ?

$>$ Ça en fait c'est parce que ma situation a changé. C'est-à-dire avant j'étais en basal-bolus et là je ne suis plus en bolus. Parce que d'après ce qu'on m'a expliqué, c'est une sorte d'étape de rémission 
partielle comme c'est appelé et je n'ai plus que la basale et du coup c'est vrai que je pense que je suis moins euh... enfin...

Sujet à des variations ?

$>$ Sujet à des variations oui.

D'accord. Est-ce qu'il y a d'autres choses que vous voudriez me dire ?

$>$ Euh...

On peut revenir sur ce que vous me disiez. Les problématiques des témoins ne sont finalement pas si proches de vos problématiques à vous. Qu'est-ce que l'on aurait pu... Quels sujets vous concerneraient plus particulièrement ?

> Moi c'est vrai que... essayer un peu d'expliquer par exemple les... je ne sais pas, les différentes phases du diabète au cours de la vie, de voir un peu comment ça peut évoluer chez certaines personnes. Par exemple moi dans mon cas j'étais à des doses assez importantes durant l'hospitalisation, après ça a continué un petit peu mais au bout d'un mois, vous voyez, j'ai arrêté euh... donc la... le bolus donc euh...

D'accord. Donc un chapitre sur l'évolution du diabète de type 1 ?

$>$ Oui.

Autre chose ?

$>$ Euh... Qu'est-ce qu'il y avait sinon... Bon le chapitre 3, je ne m'y suis pas encore euh... enfin je ne m'y suis pas intéressé. Donc j'ai fait surtout le 1 et le 2 . J'ai appris des choses. Notamment par rapport au... enfin pas les objectifs glycémiques, mais par exemple que pour... avant de conduire il fallait être au moins au-dessus de 1,20. Avant de faire du sport aussi c'était mieux d'être un peu audessus. C'est des choses que... non, que je ne savais pas.

Et qui vont vous servir ?

$>$ Oui, si.

Autre chose ?

$>$ Euh là euh non. (Rires)

Racontez-moi comment vous avez visionné le site, comment cela s'est passé ?

$>$ D'accord donc je l'ai fait déjà en une seule fois. Voilà. Donc j'ai fait les deux premiers chapitres d'un coup. Et puis bon bah je l'ai fait... Donc j'ai commencé par le chapitre 1. Après le 2, je n'ai pas... je ne pense pas avoir fait... le 2.3 par exemple « J'ai des problèmes d'hypos sévères », celuilà je ne l'ai pas fait.

D'accord. Donc vous avez vu le titre et vous vous êtes dit que ça ne vous concernait pas ?

J'ai vu les titres et j'ai sélectionné. Oui, oui.

Donc à priori vous avez fait le 1 et le 2 avec ses sous-chapitres $1,2,4$ ?

$>$ Oui.

Et le chapitre 3 vous ne l'avez pas abordé du tout ?

$>$ Le 3, non, non. Je n'ai pas...

Par manque d'envie, par manque de temps?

$>$ Manque de temps, oui, manque de temps. Enfin de temps, disons que quand je m'y suis mis euh... ça a duré à peu près... Je ne sais plus j'ai passé... Parce que j’ai vraiment tout vu quoi, même... toutes les vidéos complètement, oui j'ai mis 1 heure et demie. Donc après bon je me suis arrêté et puis c'est vrai que je ne m'y suis pas... je ne m'y suis pas replongé.

Et vous pensez y aller dans le futur ?

$>$ Oui, oui. Si, si.

Est-ce que vous l'avez regardé seul ?

$>$ Oui.

Est-ce que vous pensez que ça aurait pu intéresser des membres de votre entourage ?

$>$ Euh... Oui, je pense que ça aurait pu intéresser mes collègues et puis ma famille aussi, ma famille.

D'accord. Donc vous pensez que c'est intéressant aussi de le proposer à l'entourage ? Qu'ils peuvent apprendre des choses?

$>$ Oui, tout à fait, si.

Vous pensez en parler à quelqu'un particulièrement ?

$>$ Je suis un peu loin de ma famille. (Rires) Mais bon, pourquoi pas. Pas dans l'immédiat mais c'est une éventualité. Déjà pour eux voilà, pour voir dans quelle gamme de glycémie il faut être et puis voir un peu au niveau rythme de vie, au niveau hygiène de vie. Non je pense que c'est bien.

On va discuter du $1^{\text {er }}$ chapitre «Mes objectifs glycémiques ». Donc vous l'avez vu seul et en entier ?

$>$ Oui.

Est-ce que vous pouvez me dire en une phrase de quoi traite ce chapitre ?

$>$ Euh... Et bien il parle des objectifs euh... des objectifs, des objectifs à atteindre en terme de glycémie on va dire, une glycémie moyenne à atteindre en fait. 
Est-ce que vous pouvez me dire ce que vous avez aimé dans ce chapitre au niveau du fond, au niveau de la forme et puis éventuellement aussi ce qui était moins bien ?

> Ce que j'ai trouvé moins bien mais ça c'est plus une histoire de forme, c'est qu'il me semble qu'à un moment mais peut-être que je confonds avec le chapitre $2 \ldots$ C'est parce que... enfin on a des items à remplir. On a les hypers, donc on a un certain nombre de... par rapport à ce qu'on ressent et aussi pour les hypos. Et je crois qu'à un moment mais peut-être que c'est moi ou mon ordi je ne sais pas... mais il y a eu une sorte de bug.

Qu'est-ce qu'il s'est passé alors ?

$>$ Je voulais cocher les cases et ça ne se cochait pas. Donc je ne sais pas si c'est... Je n'ai pas réitéré.

Il y a des chiffres aussi à un moment donné à rentrer. C'est pareil vous n'avez pas pu les rentrer ?

$>\mathrm{Si}$, si ça je les ai rentrés.

Donc c'était juste les croix à cocher ?

$>$ Oui. Voilà c'était juste au niveau des croix à cocher.

Et vous n'avez pas essayé de relancer la connexion, de rafraichir ?

$>$ Si si je l'ai fait ça.

Et le problème a persisté ?

$>$ Oui, oui il a persisté, il a persisté.

D'accord. D'autres remarques sur le chapitre 1 ? Des choses que vous avez appréciées ou moins appréciées ?

> Je dirais que ce que j'ai apprécié effectivement donc ce sont... je pense que oui ce que j'ai apprécié le plus ce sont les témoignages. Les témoignages oui ça franchement j’ai apprécié.

Qu'est-ce qui vous a plu dans le fait d'entendre ces témoins ?

$>$ Déjà ça m'a permis de voir que je n'étais pas tout seul dans ce cas-là. (Rires) Donc déjà ça c'est un premier point. Et puis ça m'a permis aussi de voir un peu comment les gens vivaient avec et donc moi pouvoir essayer justement de comparer mon cas par rapport à ce que eux présentaient.

Est-ce que, alors pas forcément par rapport à ces témoignages-là, il y en a plusieurs dans le site, vous avez pu vous identifier facilement aux témoins ?

$>$ Justement, donc c'est ce que je disais juste avant, au niveau de l'identification, peut-être pas par rapport à ma situation personnelle.

Ni au niveau de ce qu'ils disent ni au niveau de qui ils sont par rapport à leur tranche d'âge... ?

$>\mathrm{Ah}$ si, si ça si tout à fait. C'est plus par rapport à moi ma situation personnelle. Mais effectivement ce que eux racontent par rapport à ce qu'ils peuvent ressentir ou peuvent vivre, ça si j'ai trouvé que c'était juste.

Est-ce que les informations contenues dans ce chapitre ont répondu aux questions que vous vous posiez sur vos objectifs glycémiques?

$>$ Oui, tout à fait.

Est-ce que vous avez des suggestions d'amélioration concernant ce chapitre ?

$>$ Non. Non.

Alors on va passer au $2^{\text {ème }}$ chapitre «Mes hypos, mes hypers ». Vous m'avez dit que vous l'aviez regardé dans l'ordre mais en passant un sous-chapitre qui vous semblait ne pas vous concerner ?

$>$ Non, mais les sous-chapitres, je ne suis pas sûr de les avoir faits dans l'ordre. J'ai sauté les hypos sévères ça c'est sûr mais je n'ai pas forcément fait dans l'ordre.

Vous êtes allé vers ce qui vous interpellait le plus?

$>$ Voilà. Donc j'ai dû faire... je crois que j'ai dû faire le 2.4 en fait « Je veux aller plus loin dans l'analyse de l'estimation de ma glycémie ».

En premier?

$>$ En premier oui. Et après j'ai dû reprendre 2.1 et 2.2.

Et e'est quelque chose qui vous semble bien, le fait de que l'on puisse faire dans le désordre, éventuellement laisser de côté, revenir ?

$>$ Oui, tout à fait, ça c'est bien.

Est-ce que vous pouvez me dire en une phrase de quoi traite ce chapitre ?

$>$ (Silence) Bah je dirais qu'il traite des situations extrêmes en terme de glycémie et donc comment repérer ces situations extrêmes et comment les prévenir quoi, comment les éviter.

Au niveau du fond, de la forme, qu'est-ce que avez-vous apprécié ?

$>$ Moi j'ai bien aimé le 2.4 (rires), celui-là il m'a vraiment bien plu. Ça vraiment j'ai bien aimé.

« Je veux aller plus loin dans l'analyse de l'estimation de ma glycémie » ? Donc c'est la grille d'analyse des erreurs. Pourquoi l'avez-vous aimé ?

$>$ C'est ça, j'ai bien aimé. Bon déjà, je suis scientifique, donc forcément j'ai été un peu plus (rires) sur ce qui est plus cartésien on va dire, plus chiffrable. Donc ça ça m'a plu. Je ne l'ai pas encore fait, mais il va falloir que je m'y mette. 
Vous pensez que ça va vous aider ?

$>$ Oui, oui. Voir à peu près si bah je suis à $5 \%$, à $20 \%$ ou à carrément hors zone si vraiment je suis à côté de la plaque.

Y a-t-il d'autres choses dans ce chapitre que vous avez appréciées ?

$>$ Euh... Dans celui-là... Oui bah c'est dans celui-là où j'ai appris justement que quand on prend la voiture il vaut mieux être au-dessus de 1.20 , quand on fait du sport il vaut mieux être aussi... il vaut mieux partir un peu plus élevé ou à 2 heures après le repas il faut être à peu près à 1.80 et à 3 heures à 1.60. C'est vrai que c'est des repères, ça a recadré un petit peu les choses. Bon après toujours les témoignages, bon à part un que j’ai trouvé qui était un peu sur joué. (Rires) Mais bon.

Dites-moi.

$>$ Bah c'était justement le cas du jeune qui balance son carnet et qui crie « Oui, j’en ai marre de ce diabète ». (Rires) Ça m'a fait rire. (Rires)

Autre chose ?

$>$ (Long silence) Euh...

Quelque chose qui vous a moins plu dans ce chapitre? Vous m'avez dit avoir eu des problèmes pour cocher des items ? Y a-t-il autre chose au niveau du fond ou au niveau de la forme ?

$>\mathrm{Au}$ niveau des hypers j'ai été étonné qu'au niveau des symptômes proposés il n’y ait pas le fait d'avoir soif. Moi, je sais que quand je suis vraiment limite, je commence à avoir la bouche sèche et avoir soif.

Vous ne l'avez pas retrouvé dans les choix proposés ?

$>$ Non.

Très bien. Autre chose ?

$>$ Non.

Est-ce que les informations contenues dans ce chapitre ont pu répondre aux questions que vous vous posiez sur vos hypos et vos hyperglycémies ?

$>$ Oui, oui.

Avez-vous des suggestions d'amélioration ?

$>$ (Silence) Non.

Le chapitre « Mes insulines », vous ne l'avez pas du tout abordé ?

$>$ Non, je ne l'ai pas du tout abordé.

Donc on a dit par manque de temps, vous n'avez pas eu le temps de vous y remettre ? Mais vous pensez quand même que vous irez le consulter ?

$>$ Oui. Si, si j'irai le consulter.

D'accord. Alors sur l'ensemble des chapitres que vous avez vus, quel est le chapitre ou le sous-chapitre qui vous a semblé le moins intéressant ou répondant le moins à des événements de votre vie quotidienne?

$>$ Bah je dirais finalement le 1 «Mes objectifs glycémiques » parce qu'ils avaient déjà été bien définis. Oui, on avait déjà bien discuté ici pendant ma semaine d'hospitalisation. Donc du coup c'est vrai que, bon c'est pas mal d'avoir un rappel hein, mais c'est vrai que du coup ce n'est pas non plus celui qui me semblait le plus nécessaire.

D'accord. Au contraire quel est le chapitre ou le sous-chapitre qui vous a le plus intéressé ou qui vous semble le plus utile pour gérer votre diabète au quotidien ?

$>$ Ah bah moi c'est clair, c'est le 2.4, ça c'est sûr.

Donc la grille d'analyse des erreurs. Est-ce qu'il y a des thèmes qui n'ont pas été abordés dans le site INDIGO et que vous aimeriez éventuellement pouvoir trouver dans le futur ?

$>$ C'est ce que j'avais un peu évoqué au début, c'était plus l'histoire, un peu les différentes possibilités d'évolution du diabète au cours de la vie. Ça ça m'intéresserait.

D'accord. Sur une échelle de 0 à 10 ; 0 : vous n'êtes pas du tout satisfait ; 10 : vous êtes très satisfait ; quelle est votre satisfaction globale vis-à-vis de l'outil INDIGO ?

$>$ Je mettrais 7 .

Qu'est-ce qu'il vous manque pour aller jusqu'à 10 ?

$>$ D'accord. (Silence) Qu'est-ce qu'il manque pour aller jusqu'à 10 ? C'est une bonne question. Qu'est-ce qu'il manque ? (Silence) Bon allez je vais dire un chapitre en plus sur l'évolution du diabète.

Est-ce que vous estimez que l'outil vous ait apporté des éléments qui allaient vous aider dans la gestion de votre diabète au quotidien ?

$>$ Oui, ah oui, ça c'est sûr.

Lesquels ? Ceux dont on a déjà parlé, les chiffres de glycémie avant de commencer le sport...

> ... Avant la conduite, estimer un peu deux heures et trois heures après la prise de repas à combien cela doit être. Voilà, et l'évaluation personnelle par rapport à l'évaluation via le lecteur... 
Est-ce que le site INDIGO va provoquer ou a provoqué des discussions entre vous et différents professionnels de santé ou membres de votre entourage? Donc membres de votre entourage on en a déjà parlé.

$>$ Voilà. Euh je pense que si vraiment il y a une discussion importante au niveau du contenu du site, je pense que ce sera plus par rapport au médecin qui me suit dans le cadre de mon diabète. Eventuellement... parce que je sais qu'au boulot on va essayer d'aller courir une fois par semaine donc peut-être qu'effectivement à ce moment-là j'aurai des choses à leur dire en disant : «Bah non là, il faut d'abord que je me resucre un peu avant d'aller courir » ou ce genre de choses. C'est plus ça.

Et avec votre médecin, votre médecin généraliste ou votre diabétologue, vous avez prévu d'aborder certains points?

$>$ Oui, oui, je pense parce que le Pr BENHAMOU m'avait dit justement d'aller suivre les chapitres, les deux premiers il m'avait dit, donc je pense que oui oui, je pense qu'on va en reparler. Tout à fait.

Vous avez une idée de ce que vous allez aborder en particulier ou du moins de ce dont vous aimeriez lui parler, des questions que vous aimeriez lui poser ?

$>$ Plus alors à ce moment-là par rapport aux hypos, aux hypos que je ne ressens pas.

Pour terminer, conseilleriez-vous le site INDIGO à un autre patient diabétique ?

$>$ Oui, bien sûr.

A un membre de votre entourage vous m'avez dit oui.

$>$ Oui.

Est-ce que vous souhaitez être informé lorsque les chapitres «Mon alimentation » et «Mes activités physiques » seront disponibles ?

$>$ Oui, tout à fait. Oui, oui, oui.

Ce sont des chapitres qui vous intéressent ?

> Bah moi ce qui m'intéresse notamment dans le 4, dans « Mon alimentation », ça c'est un truc que j'aimerais bien faire c'est... globalement c'est évaluer le nombre de glucides pris au niveau du repas pour essayer d'adapter la dose d'insuline. C'est l'insulinothérapie ça ?

Fonctionnelle.

$>$ Fonctionnelle voilà. Oui, oui, oui. Ça c'est vrai que ça m'intéresse bien ça. Même si pour l'instant, enfin tant que ça dure tant mieux, il n'y a plus de bolus. Mais ouais, si, je trouve ça intéressant.

Très bien. Est-ce que vous avez autre chose à me dire ?

Merci beaucoup.

Non, je pense que j'ai dit l'essentiel. 


\section{Entretien n ${ }^{\circ} 4$}

Quelle serait la première chose que vous auriez envie de me dire à propos du site INDIGO ?

$>$ Que je l'ai trouvé très utile pour moi que je viens de découvrir le diabète en général.

Vous avez été diagnostiqué quand ?

$>$ Le 28 de juin et je ne savais rien avant, absolument rien et donc je suis encore en train d'apprendre

\section{Par exemple?} et ça ça m'a aidé beaucoup, il a déjà répondu à beaucoup de questions.

> Par exemple je sais pas... sur la partie de l'insuline quand est-ce qu'on la fait, beaucoup de confirmations de tout qu'est-ce que j'ai appris quand j'ai fait la découverte ici à l'hôpital mais aussi, bon, on a toujours des doutes et toujours des questions et là, il m'a reconfirmé beaucoup de choses.

Ça vous a reconfirmé des choses. Ça vous a réconforté dans votre façon de prendre en charge...

$>$... Oui, bon, les contrôles glycémiques que je fais, est-ce que je les fais au bon moment, au mauvais moment ? Le bon nombre ? Les valeurs de référence? Qu'est-ce qu'on doit avoir ? Est-ce que c'est normal que des jours ce soit un petit peu plus haut que les autres ? Enfin bon, toutes ces choses...

Vous avez trouvé des informations et également ça vous a permis de vous rassurer ?

$>$ Oui. C'est plutôt les informations qui étaient absolument en ligne avec ce que j'avais moi compris, donc il a reconfirmé ça. Et donc les informations qui sont là sont... sont... Je l'ai trouvé bien utile et bien... bien utile.

Est-ce qu'il y a d'autres choses dont vous voulez parler spontanément ?

$>$ Il m'a aussi un peu stimulé pour trouver des autres questions que je vais après poser à un diabétologue la prochaine fois que je le vois.

D'accord. Vous pouvez me citer un exemple ?

$>$ Par exemple si un contrôle de glycémie est trop haut après un repas, comment je vais corriger ça ? On n'avait pas vraiment parlé avant, on n'avait pas abordé ce sujet, pas trop au moins dans la semaine que je suis resté à l'hôpital. Donc bon, ça c'est une chose que je dois bien regarder avec le docteur mais c'est une chose que j'avais pas pensé vraiment parce que, bon, ça ne m'est jamais arrivé avant.

Vous n'avez pas trouvé de réponse à cette question dans le site ou vous avez trouvé une réponse et vous avez besoin d'en parler à...

$>$... J'ai trouvé mais j'ai besoin d'en parler parce que, bon, la réponse qu'il y a sur le site c'est une réponse générale qui je pense s'applique à $99 \%$ des personnes. Pour moi, je crois, mais je n'en suis pas sûr, je crois que ça ne s'applique pas encore pour moi parce que ma dose d'insuline basale, c'est super bas, quelques unités uniquement et donc le calcul que l'on fait ça n'a pas de sens et donc je dois voir avec mon diabétologue pour comprendre comment l'appliquer à mon cas.

D'accord. Est-ce qu'il y a d'autres choses comme ça qui vous ont...

$>$... Bah ça c'est le dernier que j'ai vu. C'est vrai que le site je l'ai regardé en plusieurs séances.

Vous l'avez regardé en combien de fois ?

$>$ Je l'ai regardé peut-être... Bah le $1^{\text {er }}$ chapitre j'ai regardé tout ensemble, deux fois en plus parce que...

Vous avez regardé deux fois le $1^{\text {er }}$ chapitre ?

$>$ Oui parce que la première fois je l'ai regardé juste après que je suis sorti de l'hôpital ou presque, donc là je ne comprenais pas encore tout.

Vous pensez que vous n'étiez pas prêt à le voir ?

$>$ Non, peut-être pas prêt comme état d'esprit. Les informations j'en avais déjà, je pouvais encore peut-être l'élaborer un peu dans moi et donc... Déjà il m'a aidé dans tous les cas, parce qu'il m'a donné le parcours à suivre et après je l'ai regardé après un mois.

Après un mois ? Donc là vous vous êtes dit je retourne...

$>$... Donc j'ai recommencé à regarder tout fin août. Il y a 15 jours.

Vous avez regardé une première fois juste le $1^{\text {er }}$ chapitre ?

$>$ Oui, juste le $1^{\text {er. }}$. (Hésitation) Le docteur, il m'a donné les informations du site le 22 ou 23 de juillet. Dans la semaine d'après $j$ 'ai regardé le $1^{\text {er }}$ chapitre. Après $j$ 'ai interrompu pour un mois, en août ou... pour trois semaines, j'étais peut-être pas prêt comme état d'esprit. Les informations j'en avais déjà, je pouvais encore peut-être l'élaborer un peu dans moi. Et fin août j'ai regardé du début, j'ai regardé les chapitres 1,2 et 3.

D'accord. Et vous avez regardé les chapitres 1, 2 et 3 lors de la même séance ?

$>$ Non, non je l'ai fait dans 3-4 séances.

D'accord. Divisées par chapitre ?

$>$ Oui. En gros oui. Je pense que le chapitre 1 je l'ai fait tout d'un coup et le 2 et le 3 j'ai regardé divisé en deux. Au total cinq fois.

Avez-vous regardé les chapitres dans l'ordre : 1 puis 2 puis 3 ? 
Oui. Dans l'ordre oui.

Et à l'intérieur des chapitres est-ce que vous avez tout regardé ?

$>$ Oui. Enfin je crois. L'intention était ça.

Et est-ce que vous avez fait aussi les sous-chapitres dans l'ordre ?

$>$ Oui, l'idée était d'être sûr de ne pas louper des choses.

D'accord, pour ça ?

$>$ Plutôt pour ça et aussi l'ordre était à l'intérieur du chapitre... avait du sens donc euh...

Est-ce que vous l'avez regardé tout seul ce site ?

$>$ Oui tout seul. Aussi parce que, bon, moi je vis tout seul et ma famille elle est en Italie, elle parle pas français donc euh...

D’accord. Je voulais vous demander si vous aviez envie de le montrer à votre entourage mais...

$>\quad \ldots$ La réponse est oui mais j'ai aussi essayé, mais bon, ça ne marche pas.

Vous pensez que ça aurait pu être quelque chose qui...

$>$... Dans mon cas c'était plutôt pour rassurer ma famille. Pour qu'il peut regarder, il peut voir et avoir la confirmation que l'on peut vivre avec le diabète sans trop de soucis.

Plutôt pour leur montrer les témoignages de gens que les informations ?

$>$ Les informations techniques, non. Je pense que, dans mon cas, ce n'est pas intéressant que ma mère elle connaisse quelles sont les valeurs de glycémie. Par contre c'est plutôt intéressant pour lui dire on peut faire de l'activité physique, on peut manger qu'est-ce qu'on veut. Il n'y a pas de choses... de limitation à la vie de tous les jours. Et bon, ça je pense que si moi je le dis c'est une chose, mais si eux ils voient que le docteur ou quelque chose qui vient dans tous les cas d'un docteur, c'est plus parlant.

Vous dites que ça aurait pu rassurer vos parents sur le fait que l'on puisse bien vivre avec le diabète. Est-ce que ça vous a rassuré vous aussi dans ce sens-là ?

$>$ Pour le moment, j'ai pas besoin d'être rassuré. (Rires) J'espère de continuer comme ça.

D'accord.

$>$ A part les deux premiers jours après la découverte où j'ai été un peu déstabilisé, après tout de suite j'ai compris que c'était pas une catastrophe. Bon y a mieux (rires) mais bon. Du côté psychologique je pense ne pas avoir trop de soucis. Le site dans tous les cas ne m'a pas donné d'inquiétude ça c'est sûr.

On va parler du $1^{\text {er }}$ chapitre «Mes objectifs glycémiques ». Est-ce que vous pouvez me dire en une phrase de quoi il traite?

$>$ C'est une introduction je pense un peu générale. Comme dit le titre c'est l'objectif glycémique. Moi je... qu'est-ce que j'ai retenu un peu plus que à part le contexte global... Il y a des valeurs de référence vers lesquelles nous on doit aller. Dans le chapitre, dans le site il y a des valeurs explicites que par contre qu'il faut vérifier et un peu affiner au cas à cas.

Avec votre diabétologue ?

$>$ Exact, c'est ça. Dans tous les cas, les valeurs mentionnées sont bien liées avec quels sont mes objectifs glycémiques, pour le moment au moins. C'est une bonne confirmation. Il y a eu aussi quelques détails en plus sur des cas, comme quand on fait des activités sportives ou quand on mange plus, moins.

Ça vous a apporté des informations?

$>$ Un peu plus détaillées peut-être que ce que j'avais retenu de toutes les discussions qu'on avait eu ici (à l'hôpital).

Et ça vous a semblé utile ?

$>$ Oui, bien sûr. C'est super positif.

Y-a-t-il d'autres choses que vous avez pu apprécier dans ce chapitre ?

$>$ Là j'ai trouvé une bonne introduction à la maladie dans le sens qu'est-ce qu'on doit faire, comment se soigner. Les deux autres chapitres vont beaucoup plus dans le détail. Je l'ai trouvé bien et je pense qu'il faut avoir un chapitre comme ça pour ouvrir toutes les autres discussions.

Est-ce que vous pensez que l'on aurait pu encore l'améliorer?

$>$ Non.

Et est-ce qu'il y a des choses qui vous ont moins plu dans ce chapitre-là ?

$>$ Non, non.

Est-ce qu'il a bien répondu aux questions que vous vous posiez sur vos objectifs glycémiques ?

$>$ Oui, oui. Il répond à la question générale. Dans le cas particulier de chaque patient, c'est clair qu'il faut le regarder, le vérifier avec le diabétologue. C'est un bon démarrage pour rentrer dans le monde du diabète.

On va parler du $2^{\text {ème }}$ chapitre « Mes hypos, mes hypers ». Vous m'avez dit l'avoir vu en entier, en deux fois et dans l'ordre défini ? 
$>$ Oui.

Est-ce que vous pouvez me dire en une phrase de quoi traite ce chapitre ?

$>$ Les symptômes et qu'est-ce qu'on doit faire quand on est hypo ou hyper.

Qu'est-ce qui vous a plu dans ce chapitre, dans le contenu ou dans la forme ?

$>$ Déjà, il y a beaucoup d'exemples. Je l'ai trouvé bien, je l'ai trouvé utile. Bien sûr on peut pas donner tous les cas pour tout le monde.

Alors quel exemple ?

$>$ Je vais citer un, il y avait l'exemple pour les hypoglycémies. Le fait qu'à un certain point, si on se resucre pas quand on descend à un certain niveau, si on se resucre trop tard, à un certain point notre corps va s'adapter et donc on ne va pas les ressentir. Donc là c'est un peu mon cas. Honnêtement moi je me resucre au bon moment mais je ne les sens pas trop.

Vous ne sentez pas vos hypoglycémies?

$>$ Les symptômes parfois oui, parfois non, ça dépend. Et je n'ai pas encore compris ça dépend de quoi. Et les exemples qui sont là ils me donnent une idée de qu'est-ce que je dois regarder ou qu'est-ce que je dois faire pour essayer de ressentir plus et de ne pas entre guillemets perdre la sensibilité à ces symptômes.

Les exemples ? Est-ce que vous parlez des deux outils qui sont proposés : le carnet INDIGO et la grille d'analyse des erreurs?

$>$ Je parlais plutôt des exemples pratiques. La personne qui fait de l'activité physique et donc avec l'activité il descend trop et il ne se resucre pas ou les deux patients qui décrivent, disent qu'est-ce qu'ils font.

Ce que vous avez aimé ce sont les témoignages ?

$>$ Oui, oui. Au moins, ça il m'a aidé vraiment à retenir les informations.

Le fait que ce soit dit par quelqu'un...

$>$... Dit par quelqu'un : «Moi, je fais ça et ça et ça » et après il y a le commentaire derrière qui dit «Oui c'est bon de faire ça » ou «Non ce n'est pas correct de faire ça ».

Et vous trouvez que c'est une bonne façon de mieux mémoriser ?

$>$ Pour moi, oui.

Et vous me dites ne pas ressentir les hypoglycémies. Ce chapitre propose deux outils : le carnet INDIGO où l'on vous propose de noter les évènements de la journée, ce que vous avez mangé, si vous avez fait du sport etc.; d'estimer votre glycémie et de noter votre glycémie mesurée pour voir les écarts et aussi la grille d'analyse des erreurs. Est-ce que vous les avez utilisés ou pensez les utiliser ?

$>$ Ok. Je ne l'ai pas encore utilisé exactement comme ça. J'avais déjà avant de regarder le site noté beaucoup de choses donc c'est un peu le même esprit. Donc oui je trouve que c'est une bonne idée. Sur la grille d'erreur, moi j'avais... c'est bien. J'avais pensé à quelque chose de similaire, je suis ingénieur, les esprits s'assemblent. (Rires) La partie estimation, là ça fait pas encore partie de ma vie pour le moment. (Rires) Il y a l'analyse des résultats, l'analyse des mesures et les rapporter à qu'estce qu'il s'est passé dans la journée, là oui.

Vous avez l'intention de le faire plus tard ?

$>$ Oui, je suis en train de... Je vais en discuter aussi avec le diabétologue pour voir exactement qu'estce qu'il peut me rapporter et si c'est quelque chose qu'il faut faire, si dans mon cas c'est nécessaire de le faire plus ou moins.

Vous voulez en rediscuter avec votre diabétologue ?

$>$ Oui. Et par contre je fais déjà quelque chose un peu similaire, essayer de comprendre les variations de la glycémie elles sont dues à quoi. Ce que je disais avant sur les symptômes euh... si la glycémie est trop basse je peux penser à quelle est la raison mais, par contre je ne l'ai pas senti, je ne l'ai pas senti, je ne peux rien faire. Parfois, ça n'arrive pas tout le temps, parfois oui je le sens bien arriver et parfois non.

Vous pensez que l'outil INDIGO vous a apporté des choses pour gérer ça ?

$>$ Il y a toute la liste des symptômes connus par exemple. Oui ça aide bien sûr, ça colle bien avec les symptômes que j'ai, pas tous mais la plupart oui. Au moins pour l'hypoglycémie. Pour l'hyperglycémie honnêtement j'en ai pas ou très rarement, elles sont très basses, juste un tout petit peu au-dessus des valeurs donc je n'ai pas de symptômes. Typiquement c'est quand j'ai mangé légèrement plus. Moi je l'associe à ça. Ce n'est pas systématique et je n'ai pas de doses d'insuline à changer.

Est-ce qu'il y a des choses qui vous ont moins plu dans ce chapitre ?

$>$ Euh non. Juste la partie de l'hypoglycémie sévère. C'est bien mais, euh bon, je ne sais pas, ça ne s'applique pas encore à moi et j'espère que cela ne s'appliquera jamais. (Rires)

Ce que vous reprochez à cette partie-là c'est sa forme ou c'est le fait que pour l'instant, vous n'êtes pas confronté à ça ? 
$>$ Je ne suis pas confronté à ça et j'espère surtout de ne pas l'être mais non, non c'est informatif dans tous les cas. Pour ma situation à moi, c'est peut-être un peu trop long mais je pense que c'est bien de l'avoir dans tous les cas.

D'accord. Vous l'avez regardé finalement en entier ?

$>$ Oui, oui. Je l'ai regardé en entier, oui, oui.

D’accord. Et ça vous a apporté une information supplémentaire ?

$>$ Oui, oui. C'est juste si on veut chercher quelque chose. (Rires)

Est-ce que les informations contenues dans ce chapitre ont bien répondu aux questions que vous vous posiez à propos de vos hypos et de vos hypers ?

$>$ Oui.

Est-ce que vous avez des suggestions d'amélioration?

$>$ Non.

On va parler du $3^{\text {ème }}$ chapitre «Mes insulines ». Vous l'avez vu en entier et encore une fois en deux séances.

$>$ Oui.

Est-ce que vous pouvez me dire en une phrase de quoi traite ce chapitre ?

$>$ Comment se soigner en général. Donc euh, comment l'insuline marche, les différents types d'insuline et se soigner avec une pompe ou se soigner avec des piqures.

Et vous quel est votre mode d'injection d'insuline ?

$>$ Avec des injections au stylo.

Encore une fois, pouvez-vous me dire au niveau du contenu et de la forme, ce qui vous a plu dans ce chapitre ?

$>$ Je l'ai trouvé bien systématique on va dire. Il abordait bien tous les sujets ou toutes les questions peut-être que moi j'aimerais avoir. Il m'a aussi donné des informations que je n'avais pas, comme corriger les valeurs de glycémie s'il faut et quand il faut le faire. Et ça encore il faut bien le vérifier avec le diabétologue. C'était bien utile, bien intéressant d'avoir cette information de plus. Et les questions qui étaient là quelque part dans ma tête, ça a aidé un peu à les formaliser.

Et au niveau de la forme, il y a des choses qui vous ont plu en particulier ?

$>$ Moi je l'ai trouvé bien structuré.

Et est-ce qu'il y a des choses qui vous ont moins plu ?

$>$ Je pense que c'est difficile d'ajouter des choses. Dans le sens... je pense que le protocole de soins c'est quelque chose qu'il faut regarder de cas à cas et de personne à personne donc je ne pense pas que l'on peut mettre plus d'informations ou aller plus dans le détail parce qu'à la fin ça devient limite dangereux dans le sens que si on se calle sur ça après, bon, peut-être que pour chacun c'est différent donc euh...

Ça ne devient plus adapté ?

$>$ Oui. Donc euh c'est bien d'avoir des règles générales mais pas plus que ça je pense.

D'accord. Est-ce que vous avez des suggestions d'amélioration pour ce chapitre ?

$>$ Non. Peut-être juste, euh... je ne sais pas si c'est moi qui n'ai pas bien tout compris, peut-être qu'il manquait une information: qu'est-ce qu'il faut faire si ça ne marche pas ? C'est dans le sens l'insuline basale est censée durer $24 \mathrm{~h}$ et si on a la perception qu'elle dure, je sais pas, disons $20 \mathrm{~h}$. Il manque quatre heures : qu'est-ce que je fais? (Rires)

Qu'est-ce qui pourrait vous amener à croire ça ? Le fait que la glycémie...

$>$... La glycémie ça monte, ça monte tous les soirs un peu plus alors que le reste de la journée c'est bon et après le diner, bon bah, c'est trop haut ou avant de se coucher c'est plus haut que d'habitude et c'est quelque chose de systématique. Qu'est-ce que je dois faire dans ce cas-là ?

Vous auriez aimé avoir des réponses encore plus pratiques sur la gestion des glycémies, surtout lorsqu'elles sont à la hausse ?

$>$ Oui, peut-être oui. Mais encore peut-être que ça change trop d'un cas à l'autre donc peut-être que c'est pas facile d'insérer une information comme ça. Et en plus je ne sais pas si c'est le but. Si le but c'est juste de donner une information générale et après de discuter avec un diabétologue derrière, je pense que ça c'est une bonne chose à faire, donc là la quantité d'informations c'est bon, c'est déjà prêt. Si euh... je ne pense pas que le but c'est de remplacer le diabétologue, donc ok, c'est pas un souci.

Et donc cette question vous allez la poser à votre diabétologue ?

$>$ Oui, c'est ça.

Si l'on reprend l'ensemble des trois chapitres, quel est le chapitre ou le sous-chapitre qui vous a semblé le moins intéressant ou répondant le moins à des événements de votre vie quotidienne ?

$>$ Je l'ai dit avant, c'est les hypoglycémies sévères. J'ai dit je pense que c'est nécessaire d'avoir cette information par contre pour le moment $\mathrm{j}$ 'en ai jamais eu besoin et j'espère ne pas avoir. Il faut bien 
savoir qu'est-ce qu'on doit faire pour pas arriver là. Par contre une fois qu'on est arrivé là c'est bien de le savoir.

Au contraire quel est le chapitre ou le sous-chapitre qui vous a le plus intéressé ou qui vous a semblé le plus utile pour gérer votre diabète au quotidien ?

$>$ Ben, tous. (Rires) Le premier et le troisième chapitre sûrement. Donc les objectifs glycémiques et les insulines, donc comment se soigner. L'ordre des chapitres peut-être c'est bon, peut-être le 2 et le 3 , peut-être on pourrait les inverser. Mais ça c'est dans ma tête c'est comme ça. Après avoir donné les objectifs, peut-être on peut dire comment on peut arriver à cet objectif donc à travers l'insuline et comme troisième chapitre dire qu'est-ce qui va arriver, qu'est-ce que c'est l'hypo et l'hyper.

D’accord. Vous auriez inversé...

$>$... Parfois je pensais ça. Par contre c'est aussi vrai que c'est difficile de dire mes objectifs de glycémie c'est un certain chiffre et on ne donne pas les informations de dire qu'est-ce qui arrive si on n'est pas là, donc les hypos et les hypers donc euh...

Les deux ordres auraient pu être...

$>$ Oui. Donc jusqu'à un certain point on ne peut pas les mettre en parallèle donc euh... (Rires)

D'accord. Vous avez du mal à trancher ?

$>$ Oui honnêtement, c'est une idée, je ne sais pas. Après peut-être que si le même commentaire arrive de toutes les personnes peut-être que c'est une idée, autrement je pense que l'ordre ça va aussi comme ça.

D'accord, très bien. Est-ce qu'il y a des informations sur un thème particulier que vous n'avez pas trouvées dans le site et que vous aimeriez trouver dans le futur ?

$>$ Les chapitres 4 et 5 . (Rires)

Est-ce que vous souhaitez être informé quand les chapitres 4 et 5 seront... ?

$>$...Oui.

Ça vous intéresse, d'accord. Hormis ces deux chapitres, est-ce qu'il y a d'autres thèmes que vous aimeriez trouver ?

$>$ Je ne pense pas, je ne pense pas parce que bon ça couvre presque tout je pense.

Vous trouvez que ce sera complet avec les deux chapitres suivants ?

$>$ Oui, je pense que l'alimentation c'est une partie fondamentale de comment gérer le diabète. Et l'activité physique, moi je n'en fais pas trop mais un peu oui, mais c'est intéressant de savoir quelles sont les conséquences.

Quelle est votre satisfaction globale vis-à-vis du site INDIGO sur une échelle de 0 à 10 ; 0 vous n'êtes pas du tout satisfait ; 10 vous êtes très satisfait ?

$>$ Très satisfait parce que, bon, je trouve que c'est très intéressant, très utile de donner des informations faciles à comprendre et aussi on peut accéder à cette information de manière facile avec Internet car maintenant tout le monde est prêt, peut y arriver.

Et donc si vous deviez le chiffrer ?

$>10$.

Est-ce que vous conseilleriez ce site à une autre personne diabétique de type 1 ?

$>$ Absolument.

Et donc à votre entourage vous m'avez dit oui mais si le site était en italien.

$>$ Oui, mais bon.

Vous auriez aimé le montrer à votre famille.

$>$ Oui si ma famille parlait français. Par contre je leur ai déjà montré.

Et ils ont été intéressés vous pensez ?

$>$ Oui, ils m'ont demandé de traduire une partie, mais après bon il y a quatre heures de chapitres... Ils ne sont pas chez moi donc...

Avez-vous autre chose à ajouter ?

Merci.

$>$ Non, je ne pense pas. 


\section{Entretien n 5 :}

Quelle serait la première chose que vous auriez envie de me dire après avoir parcouru le site INDIGO ?

> J'ai beaucoup apprécié déjà parce que c'est clair, c'est complet, c'est simple à comprendre. Il y a des schémas, des illustrations. Il y a différentes activités par exemple par rapport aux schémas que l'on peut imprimer tout ça.

Vous avez trouvé que c'était interactif ?

$>$ Oui, interactif. On s'implique un peu dans le processus.

Vous vous êtes sentie impliquée dans le visionnage ? Pas simple spectatrice?

$>$ Oui, plusieurs fois par exemple ils disent : «Quelles sont les glycémies sur lesquelles vous tablez ? Les aliments que vous mettriez dans cette case ? Dans cette case ?» Enfin on participe un peu.

Vous trouvez que c'est à la fois participatif et personnalisable ?

$>$ Voilà. Ce n'est pas juste un dossier qu'on lirait, il y a moyen d'interagir avec. Ce que j'ai beaucoup apprécié aussi c'est le témoignage des personnes qui parlent, qui sont eux-mêmes diabétiques et donc on se reconnaît un peu dans eux.

Vous avez pu vous reconnaître dans les témoins ?

$>$ Oui.

Avez-vous d'autres choses à me dire spontanément ?

$>$ Hum. En fait quand il y avait les personnes qui... qui... qui participaient...

Qui témoignaient ?

$>$ Qui témoignaient, je me sentais beaucoup plus concernée en fait quand c'étaient par exemple les jeunes. Par exemple celui qui se dit «Est-ce que je vais sortir de l'amphi ou pas ? », celui qui se dit «Le carnet je n'ai pas envie de le remplir ». C'est des trucs qui me passent par la tête. Et comme les deux personnes témoins tout au long bah c'est deux personnes plutôt âgées, je me sentais beaucoup plus... je sais pas ça me... je me sentais plus concernée quand c'était la voix d'un jeune qui parlait, de par l'expérience et tout.

Il y a certains chapitres qui vous ont plus plu que d'autres parce qu'il y a certains chapitres où il y avait plus les témoignages de personnes qui vous ressemblaient et puis d'autres chapitres où il n'y avait pas de témoignages de personnes qui vous ressemblaient ?

$>$ Oui. J'ai eu le sentiment que c'était quand même principalement les deux personnes, celles qu'on voyait toujours en tandem, le monsieur qui travaille sur un tracteur et la dame. Elles sont plus âgées que moi donc euh... C'est vrai que quand c'était un jeune autour des 20 ans qui parlait c'était...

Les propos que tenaient ces deux personnes plus âgées que vous ne faisaient pas écho avec ce que vous vivez au quotidien?

$>$ Hum... Si mais pas totalement. Par exemple pour le monsieur je le trouvais plus sage entre guillemets parce qu'il faisait bien les choses et tout. Ce n'était pas vraiment la mentalité d'un jeune qui va en cours et qui fait un peu comme il peut.

D'accord. Autre chose ?

$>$ Non c'est bon.

Je vais vous demander comment vous avez visionné le site. Racontez-moi. Comment l'avez-vous consulté ?

$>$ Alors je l'ai consulté en deux fois et hum et je ne l'ai pas consulté dans l'ordre. C'est-à-dire j'ai regardé dans l'ordre des chapitres mais par contre j'allais vraiment dans ce qui m'intéressait le plus au début.

D'accord. Donc vous avez regardé d'abord le chapitre 1 puis le 2 puis le 3 mais à l'intérieur de chaque chapitre vous...

$>\quad$... Je suis allée vers ce qui me parlait le plus au début mais tout en faisant tout quoi.

D'accord donc finalement vous avez fait dans l'ordre qui vous semblait le plus intéressant pour vous mais malgré tout vous avez couvert tout le chapitre, vous les avez vu en entier ?

$>$ Oui.

Est-ce que vous vous souvenez de l'ordre qui vous a intéressée ?

$>$ Alors «Comment savoir où j'en suis au niveau de ma glycémie ? », «Je veux aller plus loin dans l'analyse de l'estimation de ma glycémie », «Comment je traite mes hypers et mes hypos ? » je n'en avais pas vraiment besoin mais je l'ai quand même regardé. " J'ai des problèmes d'hypos sévères » ça ne me concerne pas mais je l'ai regardé. Dans le chapitre $3:$ "Comment définir mes doses d'insuline ?», « Mes injections d'insuline », «Comprendre mon traitement par insuline » et «le manque d'insuline : hyperglycémie et acétone ».

Pourquoi avez-vous regardé en 2 fois ? Par manque de temps? Parce que ca vous semblait un peu «lourd » de tout faire en même temps ? Ou pour une autre raison ? 
$>$ Déjà c'était à la fin de la journée. Mais après c'est vrai que c'est un peu « lourd » de faire les trois chapitres d'un coup.

Vous auriez eu peur de ne pas tout assimiler ? De vous déconcentrer au fur et à mesure ?

$>$ Petit à petit je suivais de moins en moins donc j'ai préféré le faire en deux fois.

Est-ce qu'il y a des chapitres sur lesquels vous êtes revenue ? Est-ce qu'il y a des choses que vous avez vues plusieurs fois ou avez-vous tout vu une fois?

$>$ J'ai tout vu une fois.

Vous l'avez consulté seule ou avec quelqu'un ?

$>$ Globalement seule mais à un moment je l'ai regardé un peu avec mon frère qui est lui-même diabétique de type 1 qui a été diagnostiqué un an avant moi.

Quand a-t-on découvert votre diabète ?

$>$ Moi en décembre 2011 et lui en 2010.

Connaissait-il ce site ?

$>$ Non je lui ai fait découvrir un peu.

Qu'est-ce qu'il en a pensé ?

$>$ Il a bien aimé en fait parce que bah comme moi il y a des choses qu'il ne comprend pas tout à fait donc ça permet de faire un petit point. Il me disait : «C'est clair, c'est coloré, on peut participer, c'est animé et c'est un bon choix de support».

Vous pensez qu'il va le regarder en entier ?

$>$ Je ne sais pas. Je pense. J'ai vu qu'il avait bien apprécié je pense. Il n'avait pas tout regardé c'est vrai. Juste un peu avec moi après il est parti mais...

Vous n'en avez pas rediscuté plus que ça ?

$>$ Non sur le moment juste.

On va parler du $1^{\text {er }}$ chapitre « Mes objectifs glycémiques ». Vous l'avez regardé en entier ?

$>$ Oui.

Pouvez-vous me dire en une phrase de quoi traite ce chapitre ?

$>$ De la plage sur laquelle on préfère être. Donc par exemple quand on est à jeun, après le repas. Je pense qu'on voit le conseil de plusieurs médecins, du Pr BENHAMOU. Et on peut aussi dire quels sont nos objectifs. Combien de fois on est dans ces objectifs et... et voilà.

Qu'avez-vous apprécié dans ce chapitre ? Aussi bien au niveau du contenu que de la forme.

$>$ Alors la forme donc j'ai bien aimé que l'on puisse participer en disant que nos glycémies... enfin ce que nous on souhaite voir sur l'écran. Que l'on note tout ça, que l'on voit où on en est, ça permet de faire un point. Au niveau de ce qu'il dit je trouve qu'il est bien à la première place. C'est-à-dire qu'il est introductif, assez global. Il est un peu moins compliqué que les autres enfin je veux dire, c'est progressif je trouve.

Vous avez-trouvé que le site était bien organisé ?

$>$ Oui.

Y a-t-il des choses qui vous ont moins plu dans ce chapitre ?

$>$ Hum... Je ne crois pas. Je n'ai pas le souvenir de ne pas avoir apprécié quelque chose.

Les informations contenues dans ce chapitre ont-elles répondu aux questions que vous vous posiez sur vos objectifs glycémiques?

$>$ (Silence) Oui. Je pense que ça allait.

Avez-vous des suggestions d'amélioration pour ce chapitre ?

(Silence) Non pas vraiment. Je trouve qu'il y a l'avis des médecins, je trouve ça assez complet.

On va passer au $2^{\text {ème }}$ chapitre «Mes hypos, mes hypers ». Vous m'avez dit l'avoir vu en entier, pas forcément dans l'ordre proposé, en regardant ce qui vous interpellait le plus d'abord ?

$>$ Oui.

Pouvez-vous me dire en une phrase de quoi traite ce chapitre ?

$>$ De l'hyperglycémie et de l'hypoglycémie, de ce que l'on peut faire quand on est dans ce cas-là. (Silence) Et comprendre vraiment ce que veut dire la glycémie, c'est-à-dire à quel moment de la journée ce qu'elle signifie, comment on peut un peu essayer de savoir où on en est avant de la faire. Oui être plus attentif, mieux comprendre sa glycémie.

Vous parlez du fait d'essayer d'estimer sa glycémie avant de la mesurer. L'avez-vous expérimenté ? Est-ce que vous vous êtes servie du carnet INDIGO ? De la grille d'analyse des erreurs ? Avez-vous imprimé certaines choses ?

$>$ Alors j'ai imprimé ce qu'ils nous proposaient. Par contre je ne m'en suis par servie techniquement, je ne l'ai pas pris pour support. Mais l'idée d'estimer ses glycémies je commence à le faire, c'est-àdire avant de me piquer de me dire : «Là tu penserais que tu es à combien ? » et de vérifier.

Quels sont les résultats? 
$>$ Je ne suis pas juste du tout. J'ai souvenir d'une fois où je me croyais vraiment en hypo et j'étais plutôt haute. L'inverse aussi est arrivé. Ça m'a permis de voir en fait que je ne sentais pas forcément aussi bien que je le pensais.

Avez-vous l'impression que vos estimations s'affinent ?

$>$ Non pas encore.

Donc vous essayer d'estimer vos glycémies dans votre tête pour l'instant ? Vous ne vous êtes pas encore servie du carnet INDIGO ni de la grille d'analyse des erreurs ?

$>$ Non.

Pensez-vous vous servir de la grille d'analyse des erreurs ?

$>$ Oui j'aimerais bien. Mais comme c'est assez contraignant je pense que je vais le faire comme ils disaient, c'est-à-dire une petite semaine ou de le faire un moment bien mais je ne pourrai pas le faire tout le temps. Mais ça m'intéresse.

Tout à l'heure vous me disiez qu'il y a des choses que vous ne comprenez pas tout à fait sur le diabète. Avez-vous eu l'impression d'apprendre des choses avec ce site ?

$>$ C'est-à-dire que vu que j'avais déjà fait le stage j'avais globalement déjà tout vu et j'avais bien compris sauf que là-bas on explique et c'est clair et entre temps j'avais un peu oublié en ne retenant que l'essentiel. Et là je l'ai revu de manière aussi claire avec le support des schémas et tout. Donc euh ça m'a permis de refixer des choses que j'avais globalement comprises avant.

De refaire un point ?

$>$ Oui.

Quand avez-vous fait le stage ?

$>$ En 2012

Vous me parlez des schémas etc. Les avez-vous trouvés simples à comprendre ? Y a-t-il des choses que l'on pourrait améliorer ou est-ce que vous trouvez que c'est bien comme c'est présenté ?

$>$ Non j'ai trouvé ça clair. Ce que j'ai eu un peu de mal à comprendre c'était donc le tableau avec les couleurs...

... La grille d'analyse des erreurs ?

$>$ Voilà. Mais bon j'ai fini par comprendre mais c'est vrai qu'à la base ça fait un peu peur toutes ces couleurs et tout. Enfin je ne suis pas très... je suis littéraire donc euh... (Rires)

Finalement vous avez pris le temps, vous avez bien suivi les explications et...

$>\quad \ldots$ et on finit par comprendre.

Pensez-vous que l'on puisse améliorer cette grille d'analyse dans la façon dont on l'explique ?

$>$ Non je pense que c'est clair.

Il faut prendre le temps?

$>$ Oui. Au début on ne comprend pas tout à fait mais après oui.

Y a-t-il d'autres choses qui vous ont plu dans ce $2^{\text {ème }}$ chapitre ?

$>$ Des fois je me reconnaissais dans ce que disaient les témoins par rapport à comment ils traitaient leurs hypos et leurs hypers. Je pense bah par exemple à celui qui était dans l'amphi qui se demandait s'il allait sortir ou pas, la dame qui disait qu'elle récupérait ses hypers en faisant des bolus avant ou celui qui disait qu'il les faisait après. Donc ouais c'est des manières de faire qu'on choisit, est-ce que je me pique avant ou après ? Ça je crois que c'était peut-être après...

Oui c'est peut-être dans le chapitre 3.

$>$ Dans la pratique vu qu'ils ne font pas tous la même chose c'est vrai qu'on se reconnait un peu.

C'est la diversité des exemples vous a plu ?

$>$ Oui.

Y a-t-il des choses que vous avez trouvées moins bien dans ce chapitre ?

$>$ (Silence) Non.

Est-ce que les informations contenues dans ce chapitre ont répondu aux questions que vous vous posiez sur vos hypos et vos hyperglycémies ?

$>$ (Silence) Sur les hypers, peut-être sur la façon de les traiter. Par contre pour les hypos ça va, j'étais au point je pense.

Vous pensez avoir appris des choses sur la façon de traiter vos hyperglycémies ?

$>$ Oui, je pense, en écoutant les témoins. Par contre c'est vrai que pour les hypos je me sentais moins concernée, surtout avec «J'ai des problèmes d'hypos sévères ». Je l'ai regardé mais... vu que ça ne me concerne pas...

Sur la façon de traiter vos hyperglycémies qu'avez-vous retenu que vous ne connaissiez pas avant ?

$>$ Le problème c'est que je ne me souviens plus si je parle du $2^{\text {ème }}$ ou du $3^{\text {ème }}$ chapitre. J'ai tout confondu dans ma tête. J'ai retenu le fait que l'on pouvait se piquer avant alors que moi je rattrapais après.

Que l'on pouvait se piquer avant, c'est-à-dire ? 
$>$ Je crois que la femme disait qu'elle rattrapait avant le repas si elle était haute et qu'elle se repiquait après il me semble. Moi je ne fais pas du tout ça parce que je n'aime pas me piquer plusieurs fois. Mais c'est vrai que donc du coup quand je ne le fais pas, à la fin du repas, l'hyper plus le repas ça fait qu'on n'est pas super bien. Je vais y penser même si je n'aime pas trop me piquer plusieurs fois pour un repas.

Avez-vous des suggestions d'amélioration concernant le chapitre « Mes hypos et mes hypers »?

$>$ (Silence) Non, je ne crois pas.

On va parler du $3^{\text {ème }}$ chapitre «Mes insulines ». Je pense qu'il y a des choses que vous avez déjà dites du chapitre 3. Ce n'est pas très grave. Que pensez-vous de l'organisation des chapitres ?

$>$ (Silence) En fait comme les hypos et les hypers c'est un peu le résultat de ce que l'on fait avec l'insuline, des fois je me disais que mettre celui-ci avant c'était... au niveau des chapitres en euxmêmes...

Mettre « Mes insulines » avant « Mes hypos et mes hypers »?

$>$ Oui. Mais bon après ça risque de compliquer un peu. Là comme ça c'est vrai que c'était clair. Mais c'est vrai que je vois d'abord l'insuline et ensuite le résultat qui vient après.

D'accord. Ce chapitre vous l'avez vu en entier, en commençant par les chapitres qui vous intéressaient le plus?

$>$ Oui.

Pouvez-vous me dire en une phrase que quoi traite ce chapitre ?

$>$ Du traitement en lui-même, des insulines.

Quel est votre mode d'injection, au stylo ou à la pompe ?

$>\mathrm{Au}$ stylo. Donc il explique les deux insulines qui existent, c'est-à dire la lente et la rapide, leur fonctionnement vraiment avec des schémas, qui est vraiment différent l'une de l'autre.

Ça vous a plu la manière dont c'est expliqué ? Ça vous a appris des choses ou rappelé des choses ?

$>$ Rappelé oui parce que je l'avais déjà vu. Et donc je me disais qu'en fait c'était assez clair puisque dans mon entourage en fait j'ai une amie qui, vu que je suis diabétique, essaye de comprendre le diabète. Et j'essayais de lui expliquer donc les insulines, le comportement des insulines sur les 24 heures, et j'avais un peu de mal donc voilà. Et je me disais que ce site était clair.

Vous pensez lui conseiller d'aller le regarder ?

$>$ Oui.

Qu'est-ce qui vous a plu dans ce chapitre ?

$>$ De refaire un point. De comprendre comment ça marche ce que l'on s'injecte tous les jours. De savoir quand l'insuline est active, quand elle l'est moins ça permet aussi de comprendre ses glycémies, de savoir quand la regarder. Qu'est-ce qu'elle signifie et à quel moment.

Donc vous pensez que ce chapitre va vous aider dans la gestion de votre diabète ?

$>$ Oui, comprendre vraiment ce qui se passe et comment ça marche.

Et pouvoir adapter votre traitement en fonction ?

$>$ Oui.

Qu'est-ce qui vous a moins plu dans ce chapitre ?

$>$ (Silence) Il n'y a pas vraiment de choses qui ne m'ont pas plu je crois.

Vous m'avez dit que les informations contenues dans ce chapitre ont bien répondu aux questions que vous vous posiez sur les insulines?

$>$ Oui.

Avez-vous des suggestions d'amélioration concernant ce chapitre ?

$>$ (Silence) Non.

Si l'on reprend les 3 chapitres, quel est le chapitre ou le sous-chapitre qui vous a semblé le moins intéressant ou répondant le moins à des événements de votre vie quotidienne ?

$>$ Hum... Je dirais « J'ai des problèmes d'hypos sévères ».

Vous ne vous êtes pas sentie concernée?

$>$ Non

Au contraire, quel est le chapitre ou le sous-chapitre qui vous a le plus intéressée ou qui vous semble le plus utile pour gérer votre diabète au quotidien ?

$>$ «Je veux aller plus loin dans l'analyse de l'estimation de ma glycémie ».

Quel est le thème qui n'est pas abordé dans le site INDIGO et qui vous a manqué ? Un thème que vous aimeriez éventuellement pouvoir trouver dans le futur sur le site?

$>$ Je crois si je me souviens bien qu'il n'y a pas vraiment d'explications de comment ça se passe au niveau du sport. Comment on fait l'insuline, s'il faut en mettre la moitié... Enfin il n'y a pas...

C'est quelque chose que vous auriez aimé trouver ?

$>$ Oui. 
C'est un chapitre qui est en cours d'élaboration. Il y a deux chapitres en préparation «Mon alimentation » et «Mes activités physiques». Est-ce que vous seriez intéressée par le fait d'être informée de la disponibilité de ces chapitres sur le site ?

$>$ Oui.

Y a-t-il autre chose que vous aimeriez que l'on vous explique mieux sur le diabète et que vous n'avez pas trouvé sur le site ?

$>$ (Silence) Non.

Sur une échelle de 0 à 10, quelle est votre satisfaction globale vis-à-vis d'INDIGO : 0 vous n'êtes pas du tout satisfaite, 10 vous êtes très satisfaite ?

$>$ Je dirais 9 .

Qu'est-ce qui manquerait pour que vous puissiez mettre 10 ?

> Je dirais déjà mettre "Mes insulines » avant même si je ne sais pas si c'est possible.

D'accord donc mettre le chapitre «Mes insulines » en $2^{\text {ème }}$ et mettre le chapitre «Mes hypos, mes hypers » en $3^{\text {ème }}$ ? Vous pensez que ça serait plus clair ?

$>$ Oui. C'est-à-dire que de la manière avec laquelle je la vois c'est les hypos et les hypers qui viennent après l'insuline.

D’accord. Pour vous votre glycémie dépend de vos injections d'insuline donc ce serait plus logique d'expliquer d'abord les insulines et ensuite les hypos et les hypers ?

$>$ Oui.

Estimez-vous qu'INDIGO vous ait apporté des éléments qui allaient vous aider dans la gestion de votre diabète au quotidien ? Si oui, lesquels ?

$>$ Oui. Donc déjà l'idée d'estimer sa glycémie, que je vais essayer de faire. Et je pense que ça va améliorer des choses parce que je me suis rendu compte que je me trompais. (Silence) Je crois que c'est surtout ça en fait, l'idée de l'estimation.

Pour terminer, on a un petit peu abordé le sujet déjà. Conseilleriez-vous le site INDIGO à une autre personne diabétique de type 1 ?

$>$ Oui.

C'est ce que vous avez déjà fait avec votre frère. Vous comptez lui en reparler, lui donner les codes, etc.

Oui. J'aimerais.

Vous pensez que ça va l'intéresser ?

$>$ Oui.

Et à un autre membre de votre entourage ?

$>$ Oui. Il y a mon amie déjà.

Vous pensez à d'autres personnes que ça pourrait intéresser ou à qui vous auriez envie de montrer le site ?

$>$ Par rapport à ma famille j'aimerais bien le montrer peut-être à ma mère parce que voilà, elle ne comprend pas tout donc euh...

Pour qu'elle ait des informations supplémentaires?

$>$ Oui.

Est-ce que vous pensez que ça l'intéresserait votre maman ?

$>$ Oui.

Et vous m'avez dit que vous souhaiteriez être informée lorsque les chapitres «Mon alimentation » et « Mes activités physiques » seront disponibles?

$>$ Oui.

Avez-vous autre chose à me dire ?

$>$ Non.

Merci beaucoup.

$>$ De rien. 


\section{Entretien $n^{\circ}$ 6 :}

Quelle serait la première chose que vous auriez envie de me dire sur le site INDIGO après l'avoir exploré ?

$>$ La première chose... Non moi je l'ai trouvé bien. Parfois c'est un peu lent dans, dans... comment dire ça... dans la vidéo. Des fois elle est un peu lente, on a envie de vite passer euh...

Est-ce que c'est la navigation, le temps de chargement ou bien est-ce que c'est ce qui est dit ? Vous trouvez que ça manque de rythme?

$>$ Des fois oui voilà, comment les choses qui sont dites des fois c'est un peu lent.

D'accord. Parce que les informations vous paraissent assimilées ?

> Voilà, c'est pour ça, voilà. Mais bon, c'est de temps en temps, ce n'est pas euh...

Vous sauriez me dire à quel moment du site vous avez eu ce sentiment ?

$>$ Alors je crois que c'était dans... (silence) dans « Mes hypos, mes hypers ».

Oui. Au moment où ça parlait de quoi ?

$>$ Je crois que c'était dans « J'ai des problèmes d'hypos sévères".

Là vous avez trouvé que ça manquait de rythme ?

$>$ Oui voilà quoi. Je trouvais que en fait la mise en scène elle était bien mais en fait je me suis demandé si c'est réellement nécessaire de faire des grandes grandes mises en scène. (Rires)

Y a-t-il autre chose que vous voulez me dire spontanément sur le site INDIGO ?

$>$ La seule chose qui n'était pas rapide aussi des fois c'est quand je veux aller d'un chapitre à un autre je l'avance rapidement mais du coup j'ai l'impression que je loupe une partie. C'est pas comme les vidéos comme on a chez nous où on peut arrêter euh... Quand je commence la vidéo je me dis : «Ça je le sais. » Je veux passer à autre chose et je me dis : «Est-ce que je n'ai pas loupé quelque chose?»

Vous voulez passer une séquence mais vous ne savez pas si vous ne passez que cette séquence-là ou si vous n'en passez pas une autre en même temps ?

$>$ Voilà. Donc du coup, du coup ça m'oblige à tout regarder, vous voyez ce que je veux dire ?

Vous vous êtes sentie obligée de tout regarder pour ne rien perdre même si parfois il s'agissait de choses que vous ne vous sentiez pas obligée de regarder parce que vous les aviez déjà assimilées ?

$>$ Voilà. C'est plus dans ce sens-là.

D'accord. Alors racontez-moi maintenant comment vous avez visionné le site.

$>$ Je ne l'ai pas regardé tout d'un coup. Je l'ai regardé étape par étape. Au début je l'ai regardé... comme maintenant hein, je ne regarde que ce qui m'intéresse, sur ce que je vis en ce moment. Au tout début c'était le chapitre « Mes insulines».

Et à l'intérieur de ce chapitre « Mes insulines » vous avez tout regardé ?

$>$ Tout, oui. Ah oui, j'ai tout regardé.

Vous l'avez regardé dans l'ordre proposé ?

$>$ Oui tout à fait.

Lors de la même séance vous avez tout regardé et dans l'ordre ?

$>$ Oui.

Ensuite vous avez arrêté le visionnage. Et vous êtes revenue plus tard ?

$>$ Voilà.

Alors pour voir quoi ? Est-ce que vous avez revu des choses dans le chapitre « Mes insulines » ? Est-ce que vous avez vu un autre chapitre ?

$>$ Je crois que je suis revenue dessus, sur «Mes insulines », parce qu'il y a eu une semaine où bah voilà, $\mathrm{j}$ 'avais besoin de revoir certaines choses.

Donc pour vérifier si vous aviez bien compris, pour vous remettre des choses en tête ?

$>$ Voilà, pour m'aider à me réajuster, si j'étais dans le bon ajustement ou s'il fallait que me réajuste parce que je n'étais pas dedans.

Vous êtes-vous rendu compte qu'il y avait des choses qui vous avaient « échappé » la première fois ou finalement vous aviez tout assimilé?

$>$ Non, il y a des choses qui m'ont échappé. Il y a des choses que d'ailleurs la première fois je n'avais pas trop saisi et je me suis dit : «Bon ben, je ne vais pas repasser éternellement la vidéo. » Il faut laisser le temps aussi de le penser, le réfléchir et après y revenir plus tard.

Vous pensez que vous serez amenée à y retourner encore une ou plusieurs fois ?

$>$ Ah oui, je pense. Oui, oui.

Au gré de vos besoins?

$>$ Voilà. Tout à fait. Oui, oui. Par contre moi je trouve que c'est un outil tout à fait adapté, ben, pour moi quoi. Parce que j'ai encore besoin, même si je fais les choses correctement comme dit la diabétologue. Mais pour l'instant j'ai encore besoin d'avoir cet outil-là pour m'aider.

Ce qui vous paraît bien c'est de l'avoir sous la main, de pouvoir y retourner ? 
$>$ Tout à fait. Après il manque toujours la relation entre le diabétologue et le patient mais...

... Il y a des choses pour lesquelles vous vous êtes dit ça j’en discuterais bien ?

$>$ Voilà. Tout à fait.

Alors c'est ce que vous avez fait ? Vous avez repris rendez-vous avec quelqu'un et vous avez pu discuter du site?

$>$ Voilà j'ai pu discuter. Voilà, ouais.

Quel point avez-vous abordé ?

$>$ Eh bien là il y avait une question par rapport à mon injection ; c'est toujours par rapport aux injections d'insuline ; où j'ai eu besoin de lui demander par rapport à ce qui était dit sur le site, estce que comment moi je l'avais perçu, est-ce que j'allais faire le bon geste ? Du coup elle m'a bien confirmé que j'étais bien dans...

... Elle vous a bien rassurée ?

$>$ Bien rassurée oui. Parce que c'est vrai que je n'étais pas certaine dans ce que j'ai vu, compris.

Vous aviez besoin de l'aval d'une personne à qui vous puissiez poser directement la question ?

$>$ J'avais besoin oui, voilà.

De quand date votre diagnostic ?

$>$ Le 2 août. Euh 2 juillet je veux dire. 2 juillet de cette année.

Et vous n'êtes pas encore eu de stage d'éducation thérapeutique ?

$>$ Non, du tout.

D’accord. Est-ce que vous avez visionné le chapitre « Mes hypos, mes hypers »?

$>$ Oui.

Vous l'avez visionné de la même façon ? En une seule fois et en regardant tout dans l'ordre ?

$>$ Euh... Alors non. Je n'ai pas fait pareil. Je n'ai visionné que les deux premiers chapitres et là hier soir j'ai visionné les deux derniers.

Les deux derniers vous les aviez mis de côté parce que dans un premier temps vous ne vous sentiez pas concernée ?

$>$ Voilà, je ne me sentais pas concernée dans ça.

Et vous me dites donc que parfois vous avez eu envie de passer certaines choses...

$>\quad$... De passer, oui voilà, plus vite.

L'avez-vous fait ?

$>$ Oui je l'ai fait.

Parce que vous aviez l'impression que ces choses ne vous concernaient pas ou que vous les connaissiez déjà ?

$>$ C'est des choses que je savais.

Y a-t-il des choses que vous avez regardées plusieurs fois ?

$>$ (Hésitation) Non.

Là vous avez regardé une fois chaque sous-chapitre ?

$>$ Oui, oui. Tout à fait.

D’accord. Et le chapitre « Mes objectifs glycémiques » ?

$>$ Alors «Mes objectifs glycémiques ». Je les ai regardés. Je les trouve très intéressants mais après... Par exemple j'ai imprimé le premier chapitre sur les objectifs, ça je n'en ai pas parlé avec la diabétologue. Je ne suis pas sûre qu'à ce moment-là, euh, c'était les réponses idé... euh...

Vous ne referiez pas les mêmes réponses aujourd'hui ?

$>$ Peut-être pas. Voilà. Peut-être pas. Je suis allée un peu trop vite dans mes objectifs glycémiques. D'autant plus que j'ai même fait une erreur. A un moment donné ils demandent une question par rapport aux hypos et aux hypers, on coche plusieurs euh...

Symptômes, ressentis ?

$>$ Voilà. En fait j'ai fait une erreur parce que hypos et hypers je me suis embrouillée.

Ah. Vous avez confondu les deux ?

$>$ Voilà. Je me suis embrouillée.

Et avez-vous envie d'y retourner?

$>$ Oui. Oui, oui.

Pour revoir vos objectifs qui seraient plus proches de vos objectifs d'aujourd'hui et puis aussi refaire cette petite correction dans vos symptômes?

$>$ Tout à fait.

Et donc c'est le chapitre que vous avez visionné en dernier finalement ?

$>$ Non je crois que de suite après «Mes insulines », quelques temps après, j'ai fait « Mes objectifs glycémiques ». Je n'ai pas fait tout d'un coup. J'ai fait sur plusieurs jours, tranquillement.

Est-ce que vous avez visionné le site seule ou avec quelqu'un?

$>$ Non. Pour l'instant je l'ai visionné seule. 
Pour l'instant ? Vous avez l'intention de le montrer à quelqu'un de votre entourage ?

$>\quad$ Ah bah oui, à mon mari.

D'accord. Vous pensez que ça va l'intéresser votre mari?

$>$ Oui. Ça peut être intéressant pour qu'il comprenne réellement ce qu'est mon diabète. Parce qu'il y a des choses qu'il ne saisit pas encore totalement.

Donc dans le but de l'informer?

$>$ Oui. Tout à fait.

Vous pensez qu'il est demandeur de ça ?

$>$ Et ben pas trop. C'est pour ça que pour l'instant je lui laissais le temps d'encaisser la nouvelle. Parce que lui aussi il faut qu'il encaisse que sa femme est diabétique.

Et qu'ensuite il puisse mieux comprendre et puis éventuellement peut-être vous aider ?

$>$ Voilà. Tout à fait.

On va parler du 1er chapitre : «Mes objectifs glycémiques ». Vous m'avez-dit que vous l'aviez regardé en entier et seule. Pouvez-vous me dire en une phrase de quoi traite ce chapitre ?

$>$ (Hésitation) Ça peut me donner des indications sur les sensations que je peux avoir ou que je pourrai avoir un jour. Ça peut me donner ces indications-là.

Pouvez-vous me dire au niveau du contenu et de la forme ce que vous avez apprécié dans ce chapitre ?

$>$ (Silence) Eh bien toutes ces infos qui correspondent un peu à ce que je vis. Ça correspond à comment je vis mon diabète.

Vous avez pu vous identifier aux témoins ?

$>$ Voilà, tout à fait.

Y a-t-il des choses qui vous ont moins plu dans ce chapitre ?

$>$ Non.

On va passer au $2^{\text {ème }}$ chapitre : «Mes hypos, mes hypers ». Pouvez-vous me dire en une phrase de quoi traite ce chapitre ?

$>$ Eh bien ce sont des témoignages de personnes. Comment elles vivent leurs hypos et leurs hypers et ce qu'elles font pour... (hésitation).

Pour les corriger ?

$>$ Voilà. Tout à fait.

Qu'avez-vous apprécié dans ce chapitre ?

$>$ Et bien ce sont les témoignages moi, c'est surtout ça. Les témoignages de personnes qui m'ont permis de me dire «Bon ben elles, elles l'évaluent comme ça. »C'est important d'avoir un panel pour moi parce qu'au début je pensais qu'on était tous pareils (rires) à gérer notre diabète alors que depuis le début on me dit «ça appartient à chacun ». Et là ce témoignage a permis de balayer cette idée que j'avais, bien que j'avais eu cette info par l'équipe médicale mais elle était ancrée quand même.

Y a-t-il des choses qui vous ont moins plu dans ce chapitre ? Des choses que l'on pourrait améliorer ?

$>$ Non. Non, moi ça m'a bien plu.

Dans ce chapitre il y a le carnet INDIGO.

$>$ Voilà. Qui est bien.

Et la grille d'analyse des erreurs.

$>$ Voilà, oui.

Est-ce que ce sont des choses qui vous ont parlé ? Les avez-vous imprimées ? Pensez-vous vous en servir ?

$>$ Voilà. Je pense m'en servir. Je ne les ai pas imprimées parce qu'il fallait que je me saisisse de ça. Donc je les ai revues et là je les ai comprises et comment j'allais pouvoir les utiliser.

Est-ce que ça a été difficile à comprendre ? Pensez-vous que l'on puisse l'améliorer ?

Non ce n'est pas difficile à comprendre. Du tout. Du tout.

Vous pensez que vous allez vous en servir ?

$>$ Oui tout à fait. C'est vraiment un support qui va vraiment beaucoup m'aider.

A mieux comprendre vos symptômes et à mieux savoir où vous en êtes dans vos glycémies ?

$>$ Voilà, tout à fait. Et puis je trouve que ce qui est bien c'est qu'au début je l'ai géré avec un minimum d'infos. Aujourd'hui j'ai un peu plus d'infos et aujourd'hui je peux passer à une autre étape pour bien me saisir de mon diabète.

Avez-vous encore des questions sans réponse sur vos hypos et vos hypers après avoir visionné le chapitre?

$>$ Non.

Donc l'information était bien complète?

$>$ Oui.

Avez-vous des suggestions d'amélioration concernant ce chapitre ? 
$>$ Non. Pour l'instant non.

On va passer au $3^{\text {ème }}$ chapitre : «Mes insulines ». Donc vu l'avez vu en entier, certaines séquences plusieurs fois et c'est le premier chapitre que vous ayez visionné ?

$>$ Oui.

Pouvez-vous me dire en une phrase de quoi traite ce chapitre ?

$>$ Des insulines. (Rires) De comment adapter mon insuline par rapport à mon diabète. A quoi ça sert. Comment utiliser correctement mon stylo. Comment définir mes doses d'insuline, bien que ça c'est un peu... des fois, là j'ai un peu saisi parce que j'ai eu le rendez-vous avec la diabétologue. Donc elle m'a encore expliqué parce que c'est vrai que des fois ce n'est pas évident. Pour moi ce n'était pas évident.

Qu'avez-vous aimé dans ce chapitre ?

$>$ C'est tout cet apport d'informations qui me permettent, oui encore une fois, de faire les bons gestes parce que c'est ça le plus important pour moi : est-ce que je savais bien maitriser mon dosage d'insuline, utiliser le stylo ? Voilà, c'était tout ça.

Tout ça vous l'avez trouvé ?

$>$ Voilà.

Y a-t-il des choses dans la forme ou dans le contenu qui vous ont moins plu ?

$>$ Non.

Y a-t-il des choses que vous auriez eu envie de trouver dans ce chapitre ? Des questions que vous vous posez encore sur vos insulines?

$>$ (Silence) Au quotidien... Par exemple comment conserver la rapide et la Lantus ?

Le mode de conservation des insulines?

$>$ C'est surtout au quotidien. Là aujourd'hui je reste sur Grenoble, je vais faire mon dosage, j'ai pris mon stylo avec moi...

C'est une information que vous aimeriez pouvoir trouver ?

$>$ Voilà. Parce que ça a été quand même handicapant pour moi tout cet été. Parce que du coup moi qui a beaucoup tendance à partir toute la journée et bien l'été en pleine chaleur...

D'accord vous avez un doute sur le mode de conservation?

$>$ Oui, le mode de conservation. Ça j'ai pas trouvé cet élément là-dedans.

Il y a autre chose?

$>$ (Silence) Non. Non, non.

Avez-vous d'autres suggestions d'amélioration pour ce chapitre ?

$>$ Non. Peut-être qu'il y ait des petites infos comme ça dans notre quotidien, enfin dans mon quotidien où des fois c'est un peu difficile de trouver la réponse. On ne sait pas où pouvoir trouver la réponse. Oui comment transporter mon stylo quand je ne suis pas à la maison ? C'est des choses bêtes mais importantes pour moi.

Si l'on reprend les 3 chapitres, quel est le chapitre ou le sous-chapitre qui vous a semblé le moins intéressant ou répondant le moins à des événements de votre vie quotidienne ?

$>$ (Silence) Là, là à première vue ça serait « J'ai des problèmes d'hypos sévères ». Pour l'instant ça serait celui-là. Actuellement.

Au contraire, quel est le chapitre ou le sous-chapitre qui vous a le plus intéressée ou qui vous semble le plus utile pour gérer votre diabète au quotidien?

$>\quad$ L'analyse de l'estimation de ma glycémie.

« Je veux aller plus loin dans l'analyse de l'estimation de ma glycémie »?

$>$ Voilà. Pour moi c'est ça.

Quel est le thème qui n'est pas abordé dans le site INDIGO et qui vous a manqué ? Un thème que vous aimeriez éventuellement pouvoir trouver dans le futur sur le site ?

$>\quad$ Je n'ai pas trop réfléchi à ça. (Silence)

Des questions que vous vous posez sur le diabète et auxquelles on n'a pas encore répondu ?

$>$ Non, non, je n'ai pas trop réfléchi à ça. Ce sera plus dans le quotidien je pense. Ce seront des petites choses, oui, comment conserver mon insuline... euh...

Pour l'instant les thèmes vous conviennent bien par rapport à votre diabète aujourd'hui ?

$>$ Oui voilà.

Il y a deux chapitres en préparation « Mon alimentation » et « Mes activités physiques ».

$>$ Oui, que j'attends avec impatience. (Rires)

D'accord. Donc vous aimeriez être tenue au courant lorsqu'ils seront disponibles ?

$>$ Oui, tout à fait. C'est les premiers que j'ai consulté. J'ai vu que je ne pouvais pas y aller. (Rires) J'ai dit bon voilà...

Ce sont ceux qui vous ont attirée en premier ?

$>$ Ah oui. 
Sur une échelle de 0 à 10, quelle est votre satisfaction globale vis-à-vis d'INDIGO : 0 vous n'êtes pas du tout satisfaite, 10 vous êtes très satisfaite ?

$>$ Moi je dirais 9. Oui, oui je suis assez satisfaite quand même. Oui, oui.

D'accord. Vous mettez 9. C'est une très bonne note. Qu'est-ce qu'il vous manque pour mettre 10 ?

$>$ Moi c'est... C'est cette... rapidité là. Vous voyez ? C'est des fois pour moi... (Hésitation)

Il n'y a pas assez de rythme ?

$>$ Voilà. C'est ça qu'il me manque.

Vous vous êtes un peu ennuyée ?

$>$ Je me languissais quoi. (Rires) Je ne me suis pas ennuyée mais je me languissais... Je me disais : «Ça va arriver, ça va arriver ». Voilà quoi.

Estimez-vous que le site INDIGO vous ait apporté des éléments qui allaient vous aider dans la gestion de votre diabète au quotidien ? Si oui, lesquels ?

$>$ Ah oui, tout à fait. Ah oui, ah oui.

Pouvez-vous me dire quels éléments en particulier ?

$>$ Moi j'avais envie de dire un peu tous quand même parce qu'il y a toujours des moments où on a besoin d'avoir un outil qui... Même si ce sont des éléments qu'on connaît. Peut-être que dans un an ce ne sera pas le cas mais à l'heure d'aujourd'hui pour moi... même des fois si c'est un peu longuet je trouvais, voilà, c'est quand même important parce que ça me permet de me ressaisir du, de...

Ce que vous appréciez c'est le fait que vous puissiez y revenir n'importe quand et que vous puissiez retrouver les informations chez vous?

$>$ Ah oui, tout à fait.

Vous m'avez parlé du fait qu'il manque le dialogue avec le diabétologue. Comment pourrait-on l'améliorer?

$>$ Non pour l'instant ça va parce qu'avec le recul je me dis que c'est bien que moi je vive mon diabète, que je me pose 10000 questions qui me permettent de prendre des décisions. Parce que moi mon trait de caractère c'est que des fois je ne me fais pas confiance, $\mathrm{j}$ 'ai des doutes sur ce que je pense. Donc du coup c'est en ça que j'ai besoin d'avoir un entretien avec un diabétologue régulièrement.

Pour vous rassurer, vous confirmer le fait que vous prenez bien les bonnes décisions ?

$>$ Voilà. Et puis c'est bien aussi de pouvoir en parler, à un moment donné.

Donc en faisant comme ça, en consultant l'outil à la maison et en en rediscutant de temps en temps ça vous convient bien ?

$>$ Ah, oui. Ah oui. Tout à fait. Voilà.

Dans le questionnaire, à la question « Vous êtes vous sentie déstabilisée dans la gestion de votre diabète au quotidien ? » vous avez répondu « Oui, plutôt d'accord ». Pouvez-vous m'expliquer en quoi ?

$>$ Euh... il y a toujours un petit peu des doutes. Comment moi je vais faire... Par exemple la personne, le bûcheron, celui qui travaille dans le bois, c'est une personne qui descendait très bas dans sa glycémie et ça ça m'a beaucoup interpellée parce que je me suis demandée si ce qu'il faisait c'était bien, c'était pas bien... Bon après j'ai eu des réponses.

Dans le site ou en dehors?

$>$ Non, dans le site. J'ai eu des réponses dans le site. Mais c'est vrai qu'au tout début, avant d'aller plus loin, d'où l'importance de quand même finir un chapitre.

D'accord. Pour terminer, conseilleriez-vous le site INDIGO à une autre personne diabétique de type 1 ?

Ah oui, tout à fait.

A un membre de votre entourage? Vous m'avez dit que vous pensiez en parler à votre mari. $Y$ a-t-il d'autres personnes?

$>$ A des amis proches, qui ne saisissent pas trop de ce que je peux leur dire. Et ben : «Va voir parce que je n'arrive pas... »

Vous pensez que ça peut leur apporter des informations? Les informations dont ils peuvent avoir besoin ?

$>$ Ah oui.

Et vous pensez que c'est facilement abordable pour l'entourage ?

$>$ Oui tout à fait.

Avez-vous d'autres choses à me dire sur le site INDIGO ?

Merci.

Non. Pour l'instant non. 


\section{Entretien $\mathbf{n}^{\circ} 7$ :}

Quelle est la première chose que vous auriez envie de me dire à propos du site INDIGO après l'avoir visionné ?

$>$ Alors déjà que c'est très pédagogique, j'ai vraiment trouvé qu'il y avait même un côté vachement scolaire, ce qui est du coup très intéressant parce que quand on a des interrogations, ça permet de répondre à des questions de façon très claire, très précise.

Le coté pédagogique est apporté par quoi en particulier ?

$>$ Eh ben je trouve la forme déjà, la façon dont on clique sur les menus, voilà, les chapitres...

Vous trouvez que le site est bien structuré ?

$>$ Ah oui c'est vraiment très clair, à la limite on se pose une question : "Tiens déjà je ne sais plus, mes injections... », on va sur « Mes injections d'insuline ». Enfin, c'est très facile d'utilisation.

On peut directement aller chercher une réponse à une question que l'on se pose au bon endroit ?

$>$ Voilà. Après, j'ai trouvé que c'était quelque chose d'intéressant, parce que justement ce sont des témoignages de personnes diabétiques, donc du coup c'est vrai qu'on peut forcément se retrouver dans certaines situations.

Vous avez pu vous retrouver dans les témoins?

$>$ Voilà, au fil des témoins, il y a un évènement ou une réflexion qu'on s'est faite.

Donc dans chaque témoin finalement vous avez pu vous retrouver ?

$>$ Oui, on peut faire un petit mix et retrouver quelques situations.

Et c'est une forme qui vous a plu pour apprendre des choses ou remettre des choses au clair ?

$>$ Oui, moi je trouve que c'est... Alors moi, pour mon cas personnel, j'ai pas découvert grand chose, je pense parce que je suis passée par ici, que je sortais du stage de découverte de diabétiques de type 1.

Donc un stage d'éducation thérapeutique et c'était quand ?

$>$ C'était fin juin 2013. Donc tout ça c'était très clair pour moi. Et comme c'est ma première année de diabète je me suis beaucoup renseignée, $j$ 'ai été vraiment très bien entourée, encadrée par le personnel médical. Donc pour l'instant je suis plutôt au courant de toutes ces choses-là. Mais en revanche sur du long terme, quand peut-être on a un suivi juste avec son diabétologue où c'est peutêtre un peu moins précis, ça permet de remettre des choses à jour.

D'accord. Done vous pensez vous en servir au long cours pour aller rechercher certaines informations au moment où vous en aurez besoin ?

$>$ Oui, je pense parce qu'il s'avère que je constate que finalement ce qu'on m'avait dit mais qui me paraissait complètement aberrant ça devient réellement un automatisme.

C'est-à-dire ?

$>$ Bah le diabète, je commence à prendre des réflexes, du coup je me pose moins de questions. Et c'est là peut-être à un moment donné où on peut partir sur des directions un peu moins rigoureuses. Et ça permet, s'il y a des petits soucis au niveau de l'hémoglobine glyquée ou si on a des moments notamment d'hyper un peu trop importants, ça permet de se servir de cet outil pour euh... bon voilà, reprendre des bons réflexes. Moi je pense que c'est un outil, bon je n'irai pas le regarder sans arrêt, mais par moment ça peut être très intéressant. Et avec vraiment la curiosité pour l'alimentation...

Donc le chapitre en cours de construction ?

$>$ Oui voilà. Notamment pour compter les glucides et tout, parce que je ne suis pas encore hyper au point, je pense que ça va m'être plus utile que les questions plus générales comme ça sur mes hypos, mes hypers, comment je les traite et tout parce que ça j'ai bien compris.

Vous pensez que là vous avez vraiment des choses à apprendre et vous l'attendez ?

$>$ Voilà, voilà.

Donc, est-ce que vous souhaiteriez être informée lorsqu'ils seront disponibles ces deux chapitres ?

$>$ Ah oui, oui, oui, complètement.

D'accord.

$>$ Ensuite, j'ai bien aimé tout le côté... c'est pour ça que je vous dis que c'est très pédagogique, les explications avec les schémas, les courbes. On peut aussi rentrer ses objectifs glycémiques. Voilà, tout ça j'ai trouvé ça intéressant.

Donc le côté interactif. Vous avez trouvé que l'outil était interactif ?

$>$ Voilà, c'est interactif, c'est des fois peut-être un peu long mais bon c'est toujours pareil c'est parce que là je l'ai regardé dans une période où on m'a beaucoup dit de choses, où j'ai beaucoup été formée donc, bon, ça rajoute encore entre guillemets une couche.

D'accord. Alors vous pensez qu'il y a des choses qui manquent un peu de rythme ? Qu'il y a des parties pour lesquelles on aurait peut-être pu prendre un peu moins de temps pour les expliquer ?

$>$ Oui peut-être. En fait ça dépend à qui, enfin si c'est pour le diabétique, oui par exemple dans « Mes injections d'insuline» on voit la femme qui, donc c'est filmé assez lentement, fait sa piqûre, 
explique etc. Ça j'avais plus l'impression que ça s'adressait peut-être à l'entourage... Parce que bon, nous on fait ça tellement... 5 - 6 fois par jour ; qu'à la limite passer cinq minutes... je ne sais pas combien de temps ça dure ; sur comment faire une piqûre... Cette séquence-là pour moi ne me paraît pas particulièrement utile.

Vous vous souvenez d'une autre séquence qui vous a paru un petit peu longue ?

$>$ Un peu longue... alors... non globalement, peut-être «Comprendre mon traitement par insuline. » Mais les explications qui sont données ce n'est pas que je les ai trouvées longues, c'est que moi, étant donné que c'est clair dans ma tête... Du coup c'est vrai que ce n'est pas quelque chose qui m'a interpellée et je ne trouvais pas ça passionnant.

Est-ce que vous pouvez dire que vous vous êtes ennuyée parfois en visionnant le site ?

$>$ Ennuyée ce n'est pas le terme mais oui, j'avoue que sur « Mes insulines » j'ai trouvé ça un peu longuet. Voilà, mais bon, encore une fois je pense que c'est parce que mon diabète est très récent.

Et votre stage d'éducation thérapeutique aussi ?

$>$ Aussi. Donc je pense vraiment que c'est lié à ça. A la limite, cet outil-là si je l'avais eu en sortant de l'hôpital ça aurait certainement été assez utile pour moi. Ça m'aurait beaucoup rassurée.

Ça vous aurait beaucoup rassurée ?

> Oui. Même c'est vrai «Mes injections d'insuline » au tout début, les deux premiers mois après mon diabète, je pense que de pouvoir me référer à un site comme ça officiel ça m'aurait donné un... ça m'aurait simplement rassurée en fait.

Pour vous confirmer que ce que vous faites c'est bien la bonne façon de le faire ?

$>$ Que c'est bien et j'aurais certainement posé moins de questions peut-être à ma diabétologue ou enfin voilà. Je pense que vraiment dans la découverte d'un diabète, sortir avec le petit site en mains, je pense que c'est très important.

Donc dans le but de vous rassurer en voyant que la façon dont vous faites les choses est la bonne ?

$>$ Voilà.

Et puis peut-être également par le fait d'avoir des informations ?

$>$ Complètement parce que bon, il y a des choses que moi j'ai apprises au fil des mois et alors que là toutes les infos sont condensées. Notamment pour traiter les hypers ; bon les hypos non parce que ici ça a été très clair ; mais pour traiter les hypers je trouve que c'est très bien fait avec calculer sa dose d'insuline pour faire descendre en fonction de...

La dose de rattrapage?

$>$ La dose de rattrapage, ça je sais que j'ai mis un petit temps à être à l'aise avec cette question-là.

Ce n'est pas quelque chose que vous avez appris sur le site ?

$>$ Non, mais parce que j'ai demandé, on m'a appris et que ça fait presque un an que je suis diabétique. Du coup, par la force des choses j'ai appris à le faire mais j'ai mis très longtemps. Parce que c'est quelque chose j'étais pas du tout à l'aise, les rattrapages. J'avais un peu peur.

Est-ce que vous trouvez que la façon dont on l'explique sur le site est simple ?

$>$ Ah oui, mais vraiment je trouve que c'est un site qui est très facile d'accès, qui est très pédagogique, qui est plutôt attractif voilà.

Par?

Par les témoignages, par le rythme etc. Par les petits schémas colorés etc.

Donc aussi au niveau de la forme ?

$>$ Voilà, c'est pas du tout... parce qu'à un moment je crois qu'il y a une question sur si ça nous met mal à l'aise. Et ben pas du tout moi j'ai trouvé que ce n'est pas dans le cliché, ce n'est pas un site qui est anxiogène en fait.

D'accord et qu'avez-vous pensé du texte à l'écran ?

$>$ Très bien, enfin là j'ai vraiment euh, c'est écrit euh, au niveau de la forme ou du fond ?

De la forme.

$>$ De la forme, bah non j'ai trouvé que c'était clair, coloré.

De bonne taille, lisible ?

$>$ De bonne taille, ah oui vraiment très lisible. Je pense que même pour une personne qui n'est pas tellement habituée à se servir de l'outil Internet, c'est un site qui est très facile d'utilisation même, je pense, pour des personnes pour qui ce n'est pas forcément naturel de naviguer sur un site comme ça. Et je pense que c'est quelque chose de très important parce qu'on a justement tellement tout et rien sur Internet, c'est tellement compliqué, c'est tellement justement anxiogène avec les forums etc.

Ça vous est déjà arrivé d'être...

$>$... J'ai très vite abandonné mais je n'aime pas trop ça quoi les forums, je trouve que ce n'est pas forcément adapté parce que c'est le témoignage d'une personne. Ça peut vite faire peur parce qu'il y a de tout, enfin voilà il y a toutes sortes de situations.

Et des sites autres que des forums? 
$>$ Alors j'avoue j'ai peu fait, parce que je me méfie un peu, surtout quand il s'agit de la maladie.

Parce que vous n'étiez pas sûre de ce que vous alliez trouver ?

$>$ Voilà et souvent ça a tendance à faire un peu peur quand même, notamment quand on regarde les séquelles : «Moi il m'est arrivé ça, ça, ça ». Donc c'est vrai que j'ai plus eu le réflexe de demander à ma diabétologue, à ma généraliste qui est elle-même diabétique en plus donc euh...

Et vous trouvez que le site INDIGO est un outil qui rassure ? A aucun moment vous n'avez trouvé qu'on vous...

$>$... Jamais, ça ne fait pas peur du tout. Je pense que notamment pour le type 1 , c'est très important de nous rassurer parce que pour avoir discuté avec d'autres diabétiques de type 1 au stage, on est quand même très stressés par les séquelles éventuelles etc. Ça nous met la pression cette maladie, donc en rajouter je pense que ce n'est pas forcément bien et ce site au contraire est très clair, il ne fait pas peur. En même temps les choses sont montrées telles qu'elles sont.

Des informations ni exagérées ni sous-estimées ?

$>$ Voilà avec différents cas de figure au niveau des témoignages je trouve qu'on peut s'y retrouver car il y a différentes tranches d'âges en plus, différentes personnes.

Mais vous dites aussi que de toutes les façons finalement peu importe le profil, on retrouve dans chaque personne des choses qui rappellent des situations du quotidien ?

$>$ Oui il me semble. Voilà en fait il n'y a pas une personne qui va vraiment nous faire penser à nous mais en piochant un peu dans les différents témoignages on va se retrouver.

Et ça rassure le fait de voir que les gens vivent leur diabète soit de la même façon plutôt bien, soit au contraire de façon différente ? Qu'il y a plusieurs façons de...

$>$... Ah oui, tout à fait. Notamment je sais que par rapport aux objectifs, ils expliquaient les différents objectifs selon son mode de vie etc. Je trouve que c'est aussi intéressant de l'entendre et de se dire que c'est... Finalement en conclusion des témoignages, j’ai trouvé que le diabète finalement, les traitements d'aujourd'hui, permettent de s'adapter à différentes situations de vie et donc si même moi ma vie change pour $\mathrm{x}$ raison au fil des années, comme c'est chronique, il y aura toujours des façons d'adapter le traitement. Et ça je trouve que c'est illustré de façon indirecte à travers ces différents témoignages.

D'accord.

$>$ Donc après il n'y a pas de jeunes jeunes dans les témoignages.

D'adolescents vous voulez dire ?

$>$ Oui de $12-13-14-15$ ans, donc peut-être que c'est dommage, je ne sais pas.

Vous pensez que le support et le site pourraient intéresser cette tranche d'âge qui pour l'instant...

$>$... Oui. Notamment je pense aux parents des enfants diabétiques, je pense que c'est quelque chose qui pourrait énormément rassurer les parents. Après est-ce que l'enfant de 10 - 12 ans irait regarder ça ? Peut-être pas mais les parents qui ont vraiment besoin d'un support...

Est-ce que vous avez parlé du site dans votre entourage ? Est-ce qu'il y a des gens à qui vous voudriez montrer le site ?

$>$ Oui, bah par exemple ma mère qui est très stressée avec le diabète (rires) je pense que ça la déstresserait.

Vous pensez que ça la déstresserait par quel biais alors ?

$>$ Les témoignages notamment, où on se rend compte que les gens vont bien, qu'ils s'en sortent, qu'ils vivent bien. Et puis par rapport aux... c'est tellement pédagogique, je trouve que ça permet aux personnes de mieux comprendre les hypos, les hypers, l'insuline. Parce que souvent on me demande «Il faut en mettre plus, moins ? », «Alors si t'es en hypo ça veut dire... » Je pense que ça permet de donner des bases et ça peut être intéressant.

D'accord et vous pensez que ça serait accessible à des gens qui ne sont pas concernés par la maladie ?

$>$ Ah complètement, qui ne sont pas diabétiques. Mon compagnon a regardé un peu vite fait avec moi, pareil il a trouvé ça intéressant. Après par rapport à moi, il ne va pas regarder tous les chapitres etc. Mais ça permet de répondre à des questions.

Alors on va aborder la façon dont vous avez visionné le site. Racontez-moi.

$>$ Alors moi je suis allée directement sur « Mes hypos, mes hypers », en fait je n'ai pas suivi l'ordre du site.

D'accord, donc vous avez commencé par le chapitre 2 ?

$>$ Voilà, parce que c'est tellement la base de tout que je me suis dit « Tiens je vais voir exactement ce qu'ils disent etc. » Et ça voilà, je pense que je n'ai pas encore été confrontée par exemple à une hypo très sévère et des problèmes d'acétone etc. Mais si ça m'arrive c'est clair que j'irai me référer au site. Si j'ai un coup de stress...

D'accord. Vous les avez regardés quand même ?

$>$ Oui. 
Et vous vous dites que si jamais vous êtes effectivement confrontée à ça, vous saurez où aller chercher une information de qualité ?

$>$ Oui, voilà, exactement. Surtout que c'est des moments où on a envie tout de suite d'avoir des réponses et c'est vrai que d'aller voir sur ce site-là ça permet... Voilà je trouve que c'est très intéressant par rapport à la gestion des hypos et des hypers.

D'accord. Est-ce que dans ce chapitre-là vous avez regardé les sous-chapitres dans l'ordre qui était proposé ?

$>$ Oui là j'ai vraiment fait de façon, voilà, comme je n'étais pas confrontée au moment où je regardais à ces problèmes-là, j'ai vraiment regardé les chapitres à la suite en fait, les uns après les autres.

Est-ce que vous avez rempli les parties à cocher, à remplir ?

$>$ Alors certaines mais pas toutes. Il me semble que j'ai rempli pour... justement pour définir les doses de rattrapage. Oui, je ne sais plus où c'est, mais je sais qu'à un moment donné on peut calculer ses doses de rattrapage, en fonction de son poids etc. Ça je l'ai fait.

D’accord. Et dans « Mes hypos, mes hypers », les manifestations des hypos et des hypers ?

$>$ Voilà ah oui c'est vrai qu'il y avait tous les effets pour voir si je me reconnaissais. Donc ça aussi c'est important pour l'entourage pour les hypos. Et pour les hypers pareil, je trouve que ça permet de se rassurer en fait et d'être sûr qu'on n'est pas dans le faux par rapport à ce qu'on croit savoir.

D'accord. Dans ce chapitre-là il y a aussi le carnet INDIGO et la grille d'analyse des erreurs. Ce sont des outils dont vous vous êtes servie ou dont vous pensez vous servir ?

$>$ Non, là je ne m'en suis pas servie pour regarder le site mais pareil je pense que si à un moment donné mon diabète se déstabilise un peu, je pense que je vais vraiment me servir de ces outils-là pour remettre un peu les choses d'équerre. Par exemple, là je dois faire une hémoglobine glyquée. Si je ne suis plus dans les clous je pense que je vais approfondir ça oui.

Alors puisque l'on est sur le chapitre 2, est-ce qu'il y a des choses qui vous ont vraiment plu dans ce chapitre? Des informations qui vont vous servir au quotidien?

$>$ Non, pour l'instant non. Mais je suis sûre qu'à l'avenir oui. Là je vous dis, je pense que c'est vraiment lié au stage où j'ai vraiment fait un gros point donc du coup c'est très proche.

Est-ce qu'il y a des choses qui vous ont moins plu au niveau du fond, de la forme ? Des choses qu'on pourrait améliorer ?

$>$ Qui m'ont déplu ? Non.

Est-ce que les informations contenues dans ce chapitre ont répondu aux questions que vous vous posiez sur vos hypos et vos hypers?

$>$ Oui, au jour d'aujourd'hui, oui. Honnêtement je pense qu'il y a un peu toutes les situations, il y a l'essentiel qui est dit. Oui je trouve que c'est très bien en fait.

Très bien, alors si vous voulez bien, on va passer au chapitre « Mes objectifs glycémiques ». C'est le chapitre que vous avez regardé ensuite ?

$>$ Ensuite voilà. C'est là où je me suis rendue compte que heureusement qu'il y a plusieurs témoignages parce que ça peut être un peu... enfin chaque situation étant tellement différente, ça peut faire un peu culpabiliser si on ne se retrouve pas dans les témoignages. Parce que c'est la clé du traitement les objectifs glycémiques.

Donc est-ce que ça a été le cas ? Est-ce que ça vous a fait culpabiliser ?

$>$ Bah là par exemple, oui il me semble qu'il y a un témoignage qui disait qu'elle ne voulait jamais être au-dessus de 1 . Bon, ça si je l'avais entendu il y a quelques mois ça m'aurait vraiment fait peur.

Mais est-ce que le fait justement que l'on montre plusieurs personnes qui ont des objectifs différents...

$>\quad \ldots$ C'est très important.

C'est là qu'au contraire ça rassure ?

$>$ Ça rassure, mais en même temps, comme on veut essayer généralement de faire le mieux possible on va plutôt retenir la personne qui est la plus exigeante dans ses objectifs glycémiques, enfin c'est ce que j'ai retenu, et du coup moi je me suis dit, ouais bon, je ne suis peut-être pas assez rigoureuse dans mes objectifs glycémiques. Parce que moi l'idéal ce n'est pas d'être forcément entre 1,20 et 1,40 tout le temps.

Donc cette partie-là ne vous a pas vraiment rassurée sur vos objectifs glycémiques?

$>$ J'avoue que je l'ai regardée assez rapidement pour éviter de me retrouver face à une situation où je vais me dire «Bon ben ça va pas très bien en fait, je ne suis pas assez rigoureuse. » Même si mes hémoglobines glyquées sont correctes, n'empêche j'ai un peu le sentiment que je peux mieux faire en fait.

Est-ce que vous l'avez regardé en entier finalement ce chapitre ?

$>$ Non, ce chapitre je ne l'ai pas regardé en entier.

Il vous a déplu, il vous a mise mal à l'aise ? 
$>$ Il m'a mise mal à l'aise mais peut-être encore une fois parce que je suis dans un diabète qui est encore assez récent et voilà je ne sais pas, c'est vrai que j'avoue que...

Et est-ce que vous avez entendu les autres personnes qui avaient des objectifs plus larges ou est-ce que vous vous êtes arrêtée avant?

$>$ Oui, il y avait une dame notamment qui est infirmière et qui a des objectifs plus larges et ça m'a quand même plus rassurée. Mais c'est vrai qu'on retient la personne qui est la plus, enfin moi j'ai retenu la personne qui est la plus rigoureuse et je me suis dis que j'avais des progrès à faire, mais en même temps c'est pas mal aussi de penser ça.

D'accord. Alors pour vous qu'est-ce que l'on pourrait faire pour améliorer ce chapitre ?

$>$ Alors je ne me rappelle plus si il y avait une introduction, il me semble hein que de toutes les façons c'était vraiment en fonction des modes de vie des personnes voilà et en même temps je ne sais pas si... enfin parce que donc moi on m'a dit que pour un diabétique de type 1, enfin on me l'a dit ici (au CHU), l'idéal enfin l'idéal... oui c'était bien d'être entre 6,5 et 7,5 au niveau de l'hémoglobine glyquée. Et du coup ça correspond à une glycémie moyenne autour de, je ne sais pas, peut être de 1,60 ou quelque chose comme ça ou 1,70 .

Est-ce que vous connaissez vos objectifs ?

$>$ Ah moi oui, moi c'est entre, à jeun, entre 0,80 et 1,20 . Et ensuite...

... Ce sont des objectifs que vous avez fixés avec votre diabétologue ?

$>$ Non, en sortant du CHU et après on ne m'a plus redonné d'autres objectifs en fait. Donc voilà. Après en postprandial c'est $1.20-1.80$.

D'accord. Est-ce que finalement les informations qui étaient contenues dans ce chapitre ont répondu aux questions que vous vous posiez sur vos objectifs glycémiques?

$>$ Pas vraiment. Ouais, non voilà, j'avoue que là pas vraiment.

D’accord. On va passer au troisième chapitre « Mes insulines ». Vous avez visionné les trois chapitres lors de la même séance?

$>$ Non, j'ai fait en deux fois. Alors en premier j'ai fait « Mes hypos, mes hypers ». Et ensuite je suis allée sur « Mes insulines » donc le lendemain ou deux jours après et j'ai terminé vite fait avec « Mes objectifs glycémiques ». C'est vrai que c'est pas très logique mais euh... Mais, enfin, moi je les connais mes objectifs glycémiques, j'avais pas forcément envie d'entendre aussi peut-être des témoignages de personnes qui font mieux que moi ou je ne sais pas. (Rires) C'est possible, peut être inconsciemment... j'ai eu peur de ne pas être dans les clous du site INDIGO là-dessus.

D'accord. Alors vous m'avez dit que votre compagnon était venu de temps en temps regarder des moments avec vous?

$>$ Voilà il a regardé peut-être; je sais pas je crois que ça dure 3 heures ou un peu moins, peut-être 2 heures et $1 / 2-3$ heures ; selon comme on passe. Il a dû regarder $3 / 4$ d'heures, par bouts. Je sais qu'il a regardé «Mes injections d'insuline », il est tombé aussi sur les hypos et les hypers... Et euh si, il trouvait çà intéressant, mais plus pour moi que pour lui. Voilà.

Il a bien aimé regarder certains moments avec vous ?

$>$ Voilà.

Mais après, vous ne pensez pas qu'il va aller regarder de lui-même le reste du site INDIGO ?

$>$ Non. Non, pas mon compagnon. (Rires) Non.

D’accord. Alors est-ce que vous l'avez regardé dans l'ordre des sous-chapitres ?

$>$ Alors pareil une fois que j'allais dans les chapitres, je regardais dans l'ordre.

Et vous avez tout regardé ?

$>$ Et là j'ai tout regardé. Donc ça, j'ai trouvé ça super bien, alors là pour le coup peut-être plus pour l'entourage aussi parce que des fois, enfin pour les gens qui nous entourent ils ne comprennent pas trop comment ça marche l'insuline... Et donc ça peut être un outil, plutôt que d'expliquer, puis parfois on n'explique pas forcément les choses hyper clairement, ça peut être un outil intéressant pour la personne proche qui se pose des questions de fond sur le diabète. Moi j'ai trouvé ça très intéressant aussi. Après euh... un peu plus ennuyeux entre guillemets que le chapitre 2. Mais plus parce que c'est plus scolaire on va dire.

Voilà, notamment sur la séance où on montre une personne dans le détail qui fait son injection d'insuline?

$>$ Oui voilà. Mais pareil, je pense que quand on sort vraiment, quand on découvre ce diabète, je pense que... parce que là ça y est les piqûres c'est... Mais ça m'a fait vraiment peur au début. Et je pense que ça aurait été intéressant d'avoir ça sous la main.

D'accord. Donc si je vous demandais à quel moment vous pensez que ce site devrait être proposé à une personne diabétique?

$>$ Sortie d'hospitalisation.

En sortie de première hospitalisation? 
$>$ Ah, enfin pour moi vraiment. En sortie de première hospitalisation. Vraiment, je pense que ce serait un super support parce qu'en fait on se pose ; quand on sort de, quand on découvre le diabète de type 1 ; tout nous paraît complètement invraisemblable. Enfin rien n'est naturel.

Vous pensez être sortie de l'hôpital avec des questions encore ?

$>$ Voilà, voilà et pourtant je pense qu'ici on est quand même très très très bien pris en charge, mais malgré tout quand on est seule confrontée à la situation et moi c'est pour ça que je vous dis ça, parce que moi je me suis énormément, j’ai énormément appelé mon super ami qui est infirmier pour plein de petites questions comme ça. Et bon, tout le monde n'a pas un ami infirmier spécialisé dans le diabète.

Donc c'est cet ami qui a répondu à ces questions-là ?

$>$ Oui, mais bon parce que c'est un très bon copain, parce que j'aurais jamais appelé mon médecin comme ça ou ma diabétologue pour un oui ou pour un non on va dire. Et je pense que ça peut vraiment servir à ça, les premiers temps où tout nous paraît, rien n'est naturel en fait. Même la piqûre que là j'ai trouvé la séance pas passionnante, mais c'est vrai que...

Au début ce n'est peut-être pas comme ça que vous l'auriez vécue ?

$>\mathrm{Au}$ début, je me demandais toujours si je piquais bien, j'avais toujours peur d'oublier de faire la petite goutte au départ, donc du coup de se l'entendre dire dans « Mes injections d'insuline » je trouve que c'est quand même un chapitre en fait qui est très très important. Vraiment.

Est-ce qu'il y a d'autres choses qui vous ont particulièrement plu dans le fond ou dans la forme ?

$>$ «Hyperglycémie et acétone ». Ça en plus ça a permis, comme ça ne m’est pas encore arrivé, ça m’a permis de me remémorer ce qu'on m'avait dit à l'hôpital, sur... voilà, sur ce qu'il fallait faire, sur ce qu'il se passait quand on manquait d'insuline. Donc du coup, je l'ai trouvé assez intéressant.

D'accord. Est-ce qu'il y a des choses que vous avez trouvées moins bien dans ce chapitre ? Donc vous

m'avez dit un petit peu la lenteur, à votre stade on va dire entre guillemets, du sous-chapitre...

$>$... Voilà, mais je pense qu'il faut ce rythme-là quand on... quand on découvre un diabète de type 1, à mon avis il faut que ça aille lentement. Donc euh...

Est-ce que vous pensez à d'autres choses qui pourraient être améliorées ?

$>$ Non, comme ça non vraiment il est très bien fait. Il est un peu plus voilà, c'est un peu plus ennuyeux, c'est plus scolaire mais je l'ai trouvé très bien fait.

Est-ce que les informations contenues dans ce chapitre ont répondu aux questions que vous vous posiez sur vos insulines?

$>$ Oui. Oui, vraiment je... Voilà, oui, oui c'est très clair et voilà ça m'a permis d'être sûre que je pensais correctement en fait.

Alors de façon générale, si on reprend les trois chapitres, quel est le chapitre ou le sous-chapitre qui vous a semblé le moins intéressant ou répondant le moins à des événements de votre vie quotidienne ?

$>$ Ben du coup les objectifs glycémiques.

D'accord. Alors au contraire, qu'est-ce qui vous a semblé le plus intéressant ou le plus utile pour gérer votre diabète au quotidien?

$>$ Alors «Comment je traite mes hypers et mes hypos ».

D'accord. Est-ce que vous estimez que le site INDIGO vous ait apporté des éléments qui allaient vous aider dans la gestion de votre diabète au quotidien ?

$>$ Oui, oui. Je vais l'avoir en favori sur mon ordi et puis je pense que, pas toutes les semaines, mais je pense que je vais le regarder régulièrement. Mais, vraiment au moment de questions particulières.

Voilà, pour aller chercher des informations précises ?

$>$ Oui, ça sera vraiment quelque chose... ça m’évitera de... ben soit d'appeler mon diabétologue ou ma généraliste ; ben après si je n'arrive pas à régler le problème avec le site INDIGO, je m'en réfère à un médecin ; mais je pense que ça va être quelque chose qui va être sur mon ordinateur en favori.

Et justement est-ce que vous pensez à certaines choses qui ont été soulevées dans le site INDIGO et dont vous allez peut-être parler avec votre médecin généraliste ou avec votre diabétologue ?

$>$ Je réfléchis... Ouais, vraiment ouais la glycémie, les hypos, les hypers, je pense que ça va permettre de faire à nouveau un point et je vais parler avec ma généraliste des fameux objectifs glycémiques.

Voilà. Alors pour revoir un petit peu avec elle si elle vous confirme vos objectifs ?

$>$ Voilà. Je pense que ça sera intéressant. Mais pareil moi en fait on a fait un point pour moi au CHU il y a pas longtemps, on m'a dit que c'était bien donc... Mais je vais quand même poser la question voilà, oui c'est sûr, je parlerai un peu de ça avec ma diabétologue.

D'accord. Sur une échelle de 0 à 10 , quelle est votre satisfaction globale vis-à-vis d'INDIGO ? 0 vous n'êtes pas du tout satisfaite, 10 vous êtes très satisfaite ?

$>$ Je dirais 7 mais j'attends de voir le chapitre sur mon alimentation et les glucides. (Rires)

D'accord. Et pourquoi pas 9 ou 10 ? 
$>$ Bah parce qu'il manque peut-être dans la forme, une façon peut-être de filmer, que ce soit un petit peu plus moderne et attractif. Mais en même temps c'est un outil thérapeutique, donc ce n'est pas censé être un spot publicitaire. Mais voilà il manque peut-être, je ne sais pas quoi, mais euh...

Dans les vidéos alors?

$>$ Dans les vidéos ou euh... voilà.

Donc les vidéos, une façon de filmer un peu plus en mouvement ?

$>$ Oui un peu plus naturel, oui, voilà, voilà. Peut-être dans les témoignages c'est assez statique, mais en même temps bon je ne vois pas trop comment on peut faire autrement mais voilà c'est, je trouve que ça manque un peu de vie au niveau des témoignages.

D'accord donc les vidéos et est-ce que vous pensez à d'autres choses qui pourraient rendre le site plus attractif au niveau de la forme ?

$>$ Euh... après à part le traitement des vidéos que je trouve un peu... bon voilà, sinon au niveau des couleurs, des façons de cliquer, je trouve que c'est quand même très bien fait, très bien pensé oui.

Très bien. Pour terminer, est-ce que vous conseilleriez le site INDIGO à une autre personne diabétique de type 1 ?

$>$ Ah oui, oui, oui, oui complètement oui.

D'accord. Est-ce que vous avez quelque chose à ajouter ?

$>$ Non.

J'ai oublié une question pardon. Est-ce qu'il y a un thème qui n'est pas du tout abordé dans le site INDIGO et que vous auriez aimé trouver ou que vous aimeriez trouver dans le futur ?

$>$ Alors il me semble, un peu à travers les témoignages on le sent, mais en même temps je ne sais pas si ça serait intéressant de le mettre, c'est sur la façon dont on ressent et on vit cette maladie chronique, enfin il n'y a pas une espèce d'introduction un peu plus on va dire... psychologique.

\section{D'accord.}

$>$ Mais en même temps, il ne faut pas que ce soit trop anxiogène pour celui qui regarde donc est-ce qu'il faut forcément parler du vécu de la maladie d'une façon générale en fait ?

D’accord. Donc plusieurs témoins... Vous auriez envisagé ça sous forme de témoignages ?

$>$ Oui, oui.

Que des gens témoignent peut-être de ce qu'ils ont ressenti à l'annonce du diagnostic ?

$>$ Par exemple et de leur vécu.

A différents moments peut-être... un an après... comment...

$>\quad \ldots$ Voilà, voilà ou dix ans après. Parce que j'ai trouvé quand on a fait un tour de table à la formation de diabétiques de type 1, la première question ; on était 6 ou 7 je ne sais plus ; la première question qu'on nous a posé c'est ben voilà : «Qu'est-ce que vous avez ressenti, ça vous fait quoi d'être diabétique de type $1 »$ ? Et on a tous parlé et j'ai trouvé ça très intéressant d'entendre les témoignages d'autres diabétiques comme moi. Et en plus, on voit qu'il y a des points communs, des différences, enfin on l'a vécu de façon plus ou moins différente et voilà peut-être que ça manque un peu mais en même temps c'est aussi et surtout un outil pour essayer de mieux gérer son diabète donc je ne sais pas si c'est forcément utile, comme une espèce d'introduction, je ne sais pas.

D'accord. Donc vous l'auriez vu plutôt en début de site ?

$>$ Oui et peut-être pour sensibiliser un peu l'entourage aussi, voilà. Et du coup : «Ah ouais c'est vrai quand même c'est pas rien, du coup je vais peut-être un peu plus regarder... Un peu plus regarder, voilà, avec attention pourquoi c'est pas rien ». Parce que finalement on se rend compte que c'est quelque chose de compliqué à gérer. Donc voilà, ça c'est... voilà, peut-être pour interpeller un peu...

D'accord. Une autre suggestion?

$>$ Bah non, mis à part ça, non c'est un peu voilà, ce que j'ai ressenti en visionnant INDIGO. Mais vraiment je pense que c'est très intéressant et voilà à mon avis un diabétique de type 1 , il va s'y référer régulièrement. Avec des périodes...

Vous pensez que ce n'est pas forcément un outil que l'on visionne une fois mais plutôt un outil sur lequel on revient ?

Oui.

$>$ D'où l'importance d'être très clair dans les chapitres etc.

$>$ Et puis, c'est vrai que moi la première année, ça a été le diabète, le diabète, le diabète, donc là c'est pas forcément en ce moment où j'ai le plus envie de passer du temps sur le diabète parce que j'y ai passé toute une année de ma vie quasiment. Donc maintenant que c'est plus automatique, voilà je m'en détache un petit peu sans pour autant mal me soigner. Du coup c'est pas forcément là, à cet instant-là que je vais être la plus intéressée par tout ça, parce que c'est ce que je vous disais voilà, tout est frais, j'ai été dedans pendant un an donc... Mais au fil de ma vie c'est clair que je vais m'en servir. 
D'accord. Est-ce qu'il a des choses dans le site INDIGO que vous n'aviez abordées ni au cours de votre hospitalisation ni lors de vos trois jours d'éducation thérapeutique?

$>$ En toute objectivité, je ne pense pas. Je pense qu'on m'a quasiment tout dit. Je n'avais pas tout forcément retenu hein ça c'est sûr.

D'accord. Et donc en comparaison du stage d'éducation thérapeutique que vous avez eu, la forme vous paraît pertinente, le fait que ce soit sur Internet ?

> Ah oui, mais carrément, ouais vraiment, je pense que ça manquait en fait. Je vous dis si j'avais eu ça en sortant de l'hôpital ça m'aurait vraiment rassurée.

Voilà. Parce que c'est accessible vite ?

$>$ Oui.

De partout ?

$>$ Oui. Chez nous, facilement, enfin je trouve que ça m'aurait vraiment aidée. Et puis encore moi j'avais une personne que je pouvais appeler un peu n'importe quand, quand j'étais en panique. Mais voilà, si je n'avais pas eu cette personne-là, c'est vrai que c'est... c'est vraiment important. Et c'est vrai que là moi je suis plus dans l'alimentation maintenant.

Vous êtes en attente de ce chapitre ?

> Parce que je suis plus un peu à plus compter les glucides, j'ai plus de tableau, j'ai plus rien. Donc comme c'est quand même compliqué j'ai, ouais, j'ai vraiment envie de voir ce que ça va donner. Comment prendre en compte l'alcool, enfin voilà je serais plus, moi, dans cette phase-là du diabète maintenant.

Très bien. Est-ce que vous avez quelque chose à ajouter ?

$>$ Non.

Merci beaucoup. 


\section{Entretien $\mathbf{n}^{\circ} 8$ :}

Quelle serait la première chose que vous auriez envie de me dire après avoir exploré le site INDIGO ?

> Hum... Bah écoutez, non, c'est assez clair. La première fois que je l'avais consulté c'était juste après ma sortie d'hospitalisation, quand j'ai été diagnostiqué. Et c'est vrai que l'on a peut être besoin de... enfin ça ne sert à rien de le consulter tout de suite en fait. Il faut vraiment un peu de temps avant de pouvoir comprendre tout ce qui est expliqué. Le $1^{\text {er }}$ chapitre il est assez... en fait il ne sert pas trop quand on a été bien briefé à l'hôpital. C'est plus le 2 et le 3 qui sont plus intéressants je trouve. A mon sens.

Plus tard ou vous voulez toujours parler de tout de suite après le diagnostic ?

$>$ Non plus tard. C'est vrai que tout de suite après c'est que le $1^{\text {er }}$ qui sert. Le $1^{\text {er }}$ sert plus et le 2 et le 3 moins.

Juste en sortie d'hospitalisation le $1^{\text {er }}$ vous a intéressé et un peu plus tard ce sont les chapitres 2 et 3 qui vous ont plus plu?

$>$ Oui.

Pourquoi ?

$>$ Au niveau des... Je pense que c'est au niveau des sujets. C'est-à-dire que le $1^{\text {er }}$ est peut-être plus... basique, c'est peut-être plus général. Et c'est vrai que le 2 et le 3 il faut peut-être attendre d'avoir un peu vécu ce qui est hypers et hypos pour savoir de quoi parle le site, comprendre les témoignages. Et pour ce qui est de l'insuline ça on a un topo qui est fait en général à l'hôpital mais c'est vrai que c'est pas mal de le revoir, de refaire le point sur la durée d'action etc.

De quand date votre diagnostic ?

$>$ C'était euh... début avril 2013.

La première fois avez consulté le site quelques semaines après ?

$>$ J'ai dû le consulter... alors pas tout de suite. Le Pr BENHAMOU m'avait donné le mot de passe. Je ne l'avais pas fait tout de suite. J'avais dû l'avoir au téléphone je pense pour peut-être prendre rendez-vous ou peut-être que j'avais dû attendre un mois après lors du premier rendez-vous pour aller le voir je pense.

D'accord. Et puis là donc c'est 5 mois après que vous l'avez regardé plus attentivement ?

Oui.

La $1^{\text {ère }}$ fois vous aviez visionné quoi ? Le chapitre 1 en entier ?

$>$ La $1^{\text {ere }}$ fois, oui plus le chapitre 1 il me semble.

Et puis les autres chapitres vous vous étiez un petit peu baladé mais vous n'aviez pas regardé en entier?

$>$ Oui j'avais regardé un peu les sujets mais sans... sans plus. En fait je n’avais pas laissé défiler les vidéos. J'avais juste, j'avais utilisé le curseur pour naviguer euh... je cliquais d'une page à l'autre pour voir ce qui se disait, ce qui était écrit mais je n'avais pas pris le temps de lire les vidéos à chaque fois.

Donc on peut dire que vous avez pris connaissance de ce que contenait le site ?

$>$ Oui, voilà.

Et puis là plus récemment racontez-moi comment vous l'avez visionné. Avez-vous revu le chapitre 1 ?

$>$ Oui là par contre j'ai tout refait et à chaque fois je regardais les vidéos, enfin je faisais les pages en entier, point par point. J'ai suivi le déroulement. Et c'était pas mal. Bon après il y a deux ou trois... Je n'ai plus en tête mais c'est vrai qu'il y a certains points assez précis, quand on sait qu'on est bon dessus, par exemple, je ne sais pas moi, les valeurs glycémiques entre là où on est hyper, hypo, etc. Celles-là on les a intégrées en général donc celles-là, je zappais. Mais après c'est vrai que c'est pas mal, les témoignages par exemple sur les personnes qui ne ressentaient pas l'hypo du style le monsieur qui sentait qu'il était... que même à 0.7 par exemple il se sentait encore de courir un 100 mètres et tout, ça c'était intéressant parce que c'est vrai que l'on ne se rend pas forcément compte de jusqu'où ça peut descendre au niveau des ressentis. Ça c'était pas mal.

D'accord. Donc vous l'avez regardé dans l'ordre la deuxième fois ?

$>$ Oui. Je l'ai regardé dans l'ordre, sachant que je pense que... oui je vous dis le 1 ce n'était peut-être pas nécessaire sachant que hypers et hypos c'est repris dans le 2. Les chapitres 2 et 3 c'était bien. Et c'est vrai que les chapitres 4 et 5 ça a l'air pas mal aussi quoi, sport et alimentation ça peut être bien. J'ai eu envie d'aller voir le reste quoi.

Vous souhaiteriez être tenu au courant quand ces deux chapitres seront disponibles ?

$>$ Oui, oui, oui. Je veux bien.

Vous avez regardé le site seul ou avec quelqu'un ?

$>$ J'étais seul.

Et vous avez revu les trois chapitres en une fois ou en plusieurs fois ? 
$>$ Les trois en une fois. Ça faisait peut-être un petit peu long les trois en une fois. Deux c'est bien, trois c'est peut-être un peu long.

Vous vous êtes lassé ou vous aviez l'impression de ne pas retenir toutes les informations ?

$>$ En fait c'est que, surtout entre le 1 et le 2, il y a pas mal... on peut vraiment les prendre à part parce que j'ai l'impression qu'il y a certaines infos qui se recoupent en fait entre chaque et du coup c'est vrai que si on s'enchaine les trois à la suite ça peut faire un peu répétition quoi.

D'accord. Il y a certaines choses que vous trouvez redondantes?

$>$ Oui par exemple, (hésitation) quoi... euh et bien peut-être tout ce qui est hypers, hypos c'est vrai que ça revient souvent, les taux ça revient souvent aussi, quoi faire en cas d'hypers, d'hypos ça revient pas mal, donc euh... Ça c'est plus peut-être entre le 1 et le 2, le 3 c'est vrai que c'était plus propre à l'insuline donc ça ne revenait pas trop mais entre le 1 et le 2 c'était peut-être un peu redondant. Mais le 3 était à part donc ça passait bien.

Vous pensez que ce serait mieux de voir les chapitres 1 et 2 lors de deux sessions différentes ?

$>$ Peut-être oui. Mais après ça dépend parce que si c'est vraiment en sortie de découverte ça peut être pas mal parce que ça enfonce le clou quoi. Là je pense que c'était redondant parce qu'à mon avis depuis ma sortie j'ai intégré les taux etc. Et puis je vous dis honnêtement dans le service j'étais super bien suivi donc au niveau des taux, quoi faire, etc. Il n'y a rien sur le site où je me suis dit : «ça on ne me l'a pas dit ou ah ouais ça c'est vrai... »C'était plus un rappel en fait.

Vous avez eu l'impression que tout ce qui était dit vous le saviez déjà ?

$>$ Oui. Mais après c'est vrai que ça dépend... Oui je m'en étais souvenu quoi mais après je comprends que ça puisse être utile si quelqu'un n'intègre pas ou... parce que c'est vrai que quand on est hospitalisé au début on a pas mal de... il y a beaucoup d'infos quoi. Dès le début j'essayais de retenir parce que ça m'intéressait donc j'essayais de retenir etc. Donc...

Donc peut-être qu'il n'y avait pas de choses nouvelles mais vous avez été satisfait de les réentendre ?

$>$ Oui, oui, exactement.

On va parler du chapitre 1 : «Mes objectifs glycémiques». Qu'avez-vous apprécié au niveau du fond ou de la forme dans ce chapitre?

$>$ Tout ce qui est écrit je trouve que c'est pas mal, les schémas.

Vous avez un exemple de schéma dans le chapitre 1 ?

$>$ Hum... Le compteur glycémique c'est pas mal fait. C'est bien. Tout ce qui est écrit dans l'ensemble ça va.

Sur l'ensemble du site vous avez apprécié les schémas ? Ils étaient faciles à comprendre ?

$>$ Oui c'était bien. En fait les vidéos sont pas mal mais je pense que c'est plus euh...

... Les témoignages ?

$>$ Oui, les témoignages. A mon avis c'est plus euh... comment dire euh... on le prend plus comme soutien... soutien psychologique etc. Je trouve que ça fait plus cet effet-là. Et les textes c'est plus indicatif, c'est plus précis j'ai l'impression. Je sais que les vidéos je les regardais mais parfois c'était un tout petit peu long, du coup je zappais et c'est vrai que je m'arrêtais peut-être plus sur ce qui était écrit, schémas etc.

D'accord. Le côté témoignage, partage d'expérience ce n'est pas forcément ce que vous recherchiez ?

$>$ Si. En fait, si vous voulez je pense que l'on regarde toujours pour avoir un autre point de vue. Mais c'est vrai qu'une fois que l'on s'aperçoit que... que c'est à peu près le même ressenti que... que l'on est dans le même cadre si vous voulez, après voilà on se dit que... Je pense que l'on s'attarde plus sur... enfin pour mon cas je m'attardais plus sur les schémas précis etc. Pour approfondir le truc quoi. C'est vrai que les témoignages, je pense que les vidéos c'est peut-être plus intéressant soit pour les personnes vraiment en découverte ou qui se posent vachement de questions, ou peut-être carrément pour l'entourage qui ne connait pas du tout, là ça peut être pas mal. C'est peut-être expliqué autrement que par la personne diabétique qui en parle à son entourage.

Justement, est-ce que vous pensez que ce site qui puisse être utile à l'entourage d'une personne diabétique ?

$>$ Ouais. Ouais, je pense parce que... ouais je pense que voir quelqu'un d'autre témoigner c'est peutêtre plus... Il n'y a pas d'affectif.

Le fait que ce soit un tiers...

$>$... Oui. L'entourage du coup pourrait se rendre compte qu'il n'y a pas de modification de discours. Il n'y a pas de... on n'aggrave pas de situation ou on ne la relativise pas. Ils peuvent se rendre compte que le dialogue... enfin que c'est pareil pour les autres. Donc ça peut être pas mal.

Est-ce que vous avez pu vous identifier aux témoins ?

$>$ Euh... Ouais bah on prend un peu... Ouais forcément. Non mais il y a des choses qui... il y a des choses qui se recoupent euh... Pas un en particulier mais c'est vrai qu'il y a des situations que l'on vit dans les différents témoignages quoi. Après c'est à prendre un peu dans chaque cas. 
D’accord. Au niveau de la forme du site : les couleurs, le texte... Ça vous a satisfait ?

$>$ C'est pas mal. Par contre ça plante de temps en temps. Je sais qu'hier je n'ai pas pu y accéder par exemple. La page se chargeait tout le temps et pourtant j'utilise Safari. La première fois ça avait marché super. La $2^{\text {ème }}$ pas top, hier pas top et aujourd'hui ça va.

Le temps de chargement était un petit peu long ?

$>$ Oui et puis en fait la page n'apparaissait pas. J'avais la page d'accueil mais dès que je cliquais sur l'onglet « chapitres » euh... ça chargeait éternellement quoi si vous voulez.

Et ça vous est arrivé à deux reprises ?

$>$ Oui.

Avez-vous des problèmes similaires sur d'autres sites de temps en temps ?

$>$ Non

D’accord. Qu'avez-vous apprécié d'autre dans le $1^{\mathrm{er}}$ chapitre ? On a parlé des schémas.

$>$ (Hésitation) Les 50 mesures ça peut-être pas mal.

Quand on vous demande combien de fois vous avez été au-dessus et en-dessous de vos objectifs ?

$>$ Oui, ça peut être bien ça. Je sais que je... En ce moment je les note plus par écrit mais à l'époque où je les marquais sur un carnet papier j'aurais peut-être préféré les mettre là-dessus. Oui ça peut être pas mal d'avoir un compte-rendu par ça, pourquoi pas.

D'accord. Y a-t-il des choses que vous avez trouvées moins bien dans le $1^{\text {er }}$ chapitre ?

$>$ Euh... Non, dans l'ensemble c'est pas mal. Non c'est bien. Les vidéos peut-être un peu longues mais le fait qu'on puisse zapper à ce moment-là, il n'y a pas de soucis quoi. Le fait qu'on puisse passer d'une page à l'autre c'est bien.

Est-ce que les informations contenues dans ce chapitre ont répondu aux questions que vous vous posiez sur vos objectifs glycémiques?

$>$ Bah... Au niveau des valeurs je les connaissais. Non mais je pense que le $1^{\text {er }}$ honnêtement il est peut-être plus intéressant au niveau des témoignages qu'au niveau des chiffres. Après, tout dépend du suivi qui a été fait dans le service ou si la personne a intégré ou noté etc. Parce que c'est vrai que quelqu'un qui est peut-être plus jeune ou qui ne pense pas ou qui ne l'accepte pas etc. C'est peutêtre bien de lui rappeler. Mais moi je sais que ça s'était bien passé et donc... Les valeurs je les connaissais. Donc après c'est peut-être plus, les témoignages c'est peut-être le plus utile dans le premier.

Avez-vous des suggestions d'amélioration concernant ce chapitre ?

$>$ Non, ça m'a l'air complet.

On va passer au $2^{\text {ème }}$ chapitre : «Mes hypos, mes hypers ». Je vais vous en rappeler les 4 souschapitres : «Comment savoir où j’en suis au niveau de ma glycémie ? ", «Comment je traite mes hypers et mes hypos? ", «J'ai des problèmes d'hypos sévères » et « Je veux aller plus loin dans l'analyse de l'estimation de ma glycémie ». D'accord ?

$>$ Oui.

Ce chapitre vous l'avez vu seul, dans l'ordre défini et en entier ?

$>$ C'est ça.

Qu'avez-vous aimé dans ce chapitre ?

$>$ (Silence) Tout ce qui est hypos c'est pas mal. Vraiment le point sur les hypos et hypos sévères c'est bien puisque au moins on peut comparer symptômes et euh... La partie qui était la plus intéressante je trouve c'était celle où c'était précisé la perte de sensation au niveau du cerveau selon si on ne se resucrait pas quand on ne ressentait pas qu'on était en hypo mais que l'on était quand même en hypo au niveau des valeurs. Le fait que le cerveau se déshabituait du signal des hypos si on ne les traitait pas en-dessous de 0.7 par exemple et puis après on pouvait aller à jusqu'à 0.6 puis 0.5 puis 0.4 . Ça c'était intéressant je trouve.

D'accord.

$>$ Parce que ca m'est déjà arrivé d'être bas et de le ressentir ; ça m'est arrivé aussi d'être très bas, genre à 0.5 et de me sentir un peu fatigué mais sans avoir de transpiration ou d'être étourdi ou avoir la vue euh... Je me sentais pas mal. Donc c'est pas mal, ça motive à dire en-dessous de 0.7 tout de suite je fais quelque chose. Et le fait que ça soit réversible c'était pas mal aussi d'informer là-dessus quoi. Même si on ressent très bas, on peut faire remonter le moment déclencheur pour que le cerveau sache que... Ça c'était intéressant.

Avez-vous utilisé le carnet INDIGO ou la grille d'analyse des erreurs pour confronter vos ressentis à vos valeurs de glycémie?

$>$ Non je ne l'ai pas utilisé. Mais c'est vrai que... ça peut être intéressant. Parce que c'est vrai que c'est souvent... ça arrive de se poser des questions en se disant : «Tiens je suis peut-être bas etc. » Surtout en période irrégulière comme au début quoi. Non ça peut être bien. Mais c'est vrai qu'on a tendance de toutes les façons à le faire sans l'outil. On le fait forcément, chaque fois que l'on se 
pique on se dit : «Là je pense être à tant, à tant, à tant... » J'essaie de le faire. Après c'est vrai qu'il y a des moments : le matin ou quand on se pique machinalement on se dit...

D’accord. Y a-t-il autre chose que vous avez aimé dans ce chapitre ?

$>$ La partie sur l'hypo sévère était bien aussi. J'en ai parlé avec le Pr BENHAMOU parce que l'on parlait de la piqure, je ne sais plus pourquoi, j'ai parlé de vacances, de sport etc. Et c'est vrai que c'était pas mal de le voir en vrai, de voir comment ça se fait en vrai.

Le Glucagon?

$>$ Oui.

Vous avez trouvé que c'était explicite cette séquence ? Vous êtes rassuré maintenant ? Vous avez l'impression de savoir comment ça marche ?

$>$ Disons que les infos on me les avait déjà données, par contre il y a quelque chose que je ne savais pas c'était la durée d'action. C'est vrai que c'est peut-être pas mal d'informer l'entourage. Parce que bon j'ai informé mon entourage en disant : «En cas de pépin il faut faire ci, il faut faire ça » mais c'est vrai que le fait d'informer sur le fait que ça puisse durer dix minutes un quart d'heure avant que l'on reprenne conscience ça peut être pas mal parce que bon, ça évite à la personne d'être en stress pendant dix minutes pour rien quoi.

Est-ce que c'est une séquence que vous envisagez de montrer à vos proches ?

$>$ Euh... pourquoi pas ouais. Je pense oui, c'est peut-être la plus, en premier à choisir comme ça, c'est peut-être la plus parlante effectivement oui pour l'entourage.

D'autres points positifs ?

$>$ Ah bah écoutez non. Dans l'ensemble je vous dis tout est... Je n'ai pas de... Tout m'a semblé bien et puis en particulier ça quoi.

D'accord. Donc dans le chapitre 2 il n'y a rien que vous ayez trouvé moins bien ?

$>$ Non.

Avez-vous des suggestions d'amélioration concernant ce chapitre ?

$>$ Ça m'a l'air bien complet.

Est-ce que les informations contenues dans ce chapitre ont répondu aux questions que vous vous posiez sur vos hypos et vos hyperglycémies?

$>$ Peut-être plus, oui, plus que le chapitre 1.

Vous avez eu des réponses à toutes les questions que vous pouviez vous poser ?

$>$ Dans l'ensemble oui.

On va passer au $3^{\text {ème }}$ chapitre : «Mes insulines ». Il est composé de sous-chapitres qui sont : «Comprendre mon traitement par insuline », « Mes injections d'insuline », « Comment définir mes

doses d'insuline ? » et « Le manque d'insuline : hyperglycémie et acétone ». D'accord ?

$>$ Oui.

Vous l'avez vu en entier, seul et dans l'ordre?

$>$ Oui.

Qu'avez-vous aimé dans ce chapitre ?

$>$ Tout le rappel sur euh... Ah non en fait ce qui m'a bien intéressé c'était le schéma du corps humain avec... sur quels organes agissait l'insuline, dans quel ordre, dans quelle situation etc. Ça c'était bien parce qu'on me l'avait fait à l'hôpital mais vu que c'est assez complexe c'était pas mal de le revoir. Et notamment l'info qui précisait que déjà c'est vraiment dissocié, que la lente agissait principalement sur le foie, enfin sur la libération de sucre qui était faite dans le foie et qu'il était possible de jeûner, de sauter un repas.

Y a-t-il autre chose qui vous ait plu dans ce chapitre ?

$>$ Non. Comme ça de tête...

Qui vous ait déplu ?

$>$ Non, je n'ai rien qui me vient à l'idée, c'est bien, c'est complet.

Les informations contenues dans ce chapitre ont-elles répondu aux questions que vous vous posiez sur vos insulines?

$>$ Oui.

Avez-vous des suggestions d'amélioration ?

$>$ Non, franchement c'est bien complet.

Si l'on reprend les trois chapitres, quel est le chapitre ou le sous-chapitre qui vous a semblé le moins intéressant ou répondant le moins à des événements de votre vie quotidienne ?

$>$ (Silence) Là comme ça de tête... (Silence) Peut-être ce qui est plus général. Peut-être dans le 1, peut-être le début du 1, tout ce qui reprend vraiment les taux en-dessous desquels il ne faut pas être, les taux au-dessus desquels il ne faut pas être etc. Ça en général, vu que c'est quelque chose quand même d'important qu'on utilise tous les jours, ça en général on le sait quoi. On va dire vraiment les 
bases ; ce qui nous a déjà été dit à l'hôpital à la limite c'est peut-être ce qui serait le moins utile dans le chapitre 1 mais après le reste franchement, tout est bien, enfin c'est bien de le rappeler quoi.

Au contraire, quel est le chapitre ou le sous-chapitre qui vous a le plus intéressé ou qui vous semble le plus utile pour gérer votre diabète au quotidien ?

$>$ Le chapitre sur les insulines je pense que c'est le plus intéressant.

Parce que le plus pratique finalement ?

$>$ Le plus pratique et puis c'est vrai que tout ce qui est hypos et hypers c'est vraiment du ressenti, bon à part les infos que je vous ai précisées qui étaient intéressantes, mais après c'est vraiment quelque chose qu'on vit seul donc à la limite on consulte mais après on met en application seul. Mais « Mes insulines » je pense que ça peut éviter pas mal de mails ou de questions pas forcément utiles dans un premier temps à notre diabéto parce que c'est des choses...

D'accord. Pensez-vous discuter de certaines choses avec votre diabétologue ? Y a-t-il des choses que vous aimeriez revoir avec lui ?

$>$ Non c'était tout clair. Mais justement à l'inverse, par exemple le chapitre sur les insulines ; je lui ai envoyé un mail il y a quelques jours parce que j'avais une modification de taux ; et c'est vrai que je pense qu'en consultant, je ne sais plus quel point c'était exactement mais... qui faisait le point entre la basale, la rapide, qu'est-ce que ça pouvait être si le taux se modifiait, euh... voilà ça peut être bien de le voir, même en le voyant sans l'appeler ou sans le contacter par mail, ça peut suffire dans un premier temps et puis ça peut faire patienter jusqu'à la prochaine visite.

Vous pensez que vous auriez pu répondre à votre question en visionnant ce chapitre ?

$>$ Au moins en partie et du coup peut-être patienter jusqu'à la visite mensuelle ou bimensuelle.

D'accord. Alors y a-t-il un thème, un sujet qui n'est pas abordé dans le site INDIGO et que vous aimeriez trouver dans le futur ?

$>$ (Silence) Alimentation et activités physiques ça sera bien, ça sera complet. Hum, si peut-être je pense ce qui peut être bien c'est peut-être un pré-chapitre avant le 1 du style causes d'apparition ou quelque chose comme ça avec des stats, je pense que ça peut être pas mal de donner des chiffres ou ce qui peut être cause d'apparition du diabète, ça peut être bien pour informer à mon avis.

Des statistiques sur quoi, sur le nombre de personnes atteintes, l’âge ?

$>$ Des chiffres. Des chiffres ça peut être bien.

Des chiffres précisant quoi exactement?

$>$ Des chiffres par exemple sur le nombre de diabétiques de type 1, de type 2. Donner peut-être des chiffres quant à l'âge d'apparition, les causes d'apparition. Je pense que ça peut bien aller avec les témoignages du $1^{\mathrm{er}}$ chapitre pour les personnes qui sont en découverte, ça peut être bien. Et peut-être aussi un chapitre sur les différents euh... il y a déjà les différents types d'insuline, mais peut-être sur les différents modes de traitement du style injections, pompe. Peut-être une présentation des pour et des contre de la pompe, de l'injection. Je sais que c'est abordé dans les insulines mais peut-être... Oui un chapitre à part entière carrément sur les modes de traitement existants du style pompes, injections, indicateurs glycémiques. Enfin donner un peu des infos qui peut-être justement dégrossiraient l'information avant de poser des questions à notre diabéto en consultation quoi.

Quel mode de traitement avez-vous ?

$>$ Là je suis en période de lune de miel, du coup je suis en injections, j'en fais très peu donc toujours au stylo quoi. Et puis peut-être un point sur la recherche éventuellement, les avancées, ça peut être bien. Vu que c'est un secteur qui est... enfin je sais que pour le diabète c'est assez dynamique. C'est peut-être bien d'informer. Parce que c'est vrai que les secteurs qui avancent peu c'est peut-être pire qu'autre chose qu'on voit que ça ne soit jamais actualisé etc. Mais là c'est assez dynamique c'est peut-être pas mal de tenir au courant de l'évolution.

Pensez-vous que dans le futur vous pourrez être amené à retourner sur le site rechercher des informations ou reconsulter des points précis ?

$>$ Oui, je pense que oui c'est peut-être bien de l'avoir justement sous forme de site. Le nom on s'en souvient bien, les codes d'accès aussi. Donc je pense que plutôt que d'avoir un papier ou un carnet que l'on va peut-être perdre ou on ne saura pas où il est euh... ouais du coup on se dit : « oui bah tiens je vais peut-être aller chercher une info, je ne suis pas sûr, jeter un œil, voir si c'est abordé ». Oui je pense, ça peut être réutilisé.

Sur une échelle de 0 à 10, quelle est votre satisfaction globale vis-à-vis du site INDIGO : 0 vous n'êtes pas du tout satisfait, 10 vous êtes très satisfait ?

$>$ Je ne sais pas, je dirais... allez 8 .

Et qu'est-ce qu'il vous manque pour mettre 9 ou 10 ?

$>$ Je dirais le fait qu'il a planté deux fois enfin que je n'ai pas pu m'en servir deux fois, je n'arrivais pas sur la $2^{\text {ème }}$ page. Et puis après peut-être dans la présentation, peut-être un tout petit peu plus sobre, moins de fonctions. Parce que c'est vrai que tous les petits onglets sous la vidéo à part le 
bouton «avancer » et « reculer » je trouve que ce n'est pas toujours utile quoi. Parce qu'à la limite qu'il y ait la possibilité de mettre en plein écran etc. on ne l'utilise pas trop. On peut laisser comme c'est quoi.

C'est lisible sans mettre en plein écran ?

$>$ Oui je pense que ça suffit puisque vu que de toutes les façons ça laisse... enfin du coup ça laisse accès au menu, aux onglets sur les côtés etc. Je trouve que c'est peut-être même pas plus mal de ne pas le mettre en plein écran et du coup enlever même par exemple le bouton « volume », il n'y a pas tellement besoin parce qu'à la limite le volume on peut le régler de l'ordinateur. Faire un petit peu plus sobre du coup, enlever ce qu'on peut enlever quoi.

D'accord. Estimez-vous qu'INDIGO vous ait amené des éléments qui allaient vous aider dans la gestion de votre diabète au quotidien?

Lesquels ?

Oui. Je dirais que oui. Certains.

$>$ Je dirais (Hésitation) Lesquels ? Peut-être tout ce qui est gestion des hypos et peut-être calcul de l'insuline aussi ça peut être bien.

Gestion des hypos ? Vous voulez-dire..

$>\ldots$ Tout ce qui est ressenti et le fait qu'on puisse ressentir de plus en plus tard etc.

Pour terminer conseilleriez-vous le site INDIGO à une autre personne diabétique de type 1 ?

$>$ Oui. Oui parce que de toute façon ça ne fait pas de mal de revoir les infos une seconde fois.

Conseilleriez-vous le site INDIGO à un membre de votre entourage?

$>$ Aussi oui. La partie dont on a parlé sur le Glucagon ça peut être bien.

Vous en avez parlé avec votre famille, vous aviez envisagé de leur montrer le site ou pas ?

$>$ Oui, sans problème.

Vous pensez que ça va les intéresser ?

$>$ Je pense oui.

Avez-vous autre chose à ajouter ?

$>$ Non, je crois que c'est complet.

Merci beaucoup. 


\section{Entretien n ${ }^{\circ}$ :}

Quelle serait la première chose que vous auriez envie de me dire après avoir exploré le site INDIGO ?

$>$ Euh... Qu'il est très bien pensé enfin, c'est bien fait, c'est interactif. Ça permet d'aborder la chose... C'est sympa contrairement à tout ce qui peut être support papier qui fait tout de suite plus formel. Je trouve que ça donne plus envie parce que j'ai tendance à ne pas trop vouloir y penser, me pencher dessus alors que c'est quand même quelque chose d'important qu'il faut que je fasse. Là ça m'a permis un peu de me plonger dedans sans avoir l'impression de le faire.

D'accord. Grâce au fait que ce soit sur Internet ou à la forme du site ?

$>$ Non, la forme du site. Ça fait un peu site pour enfants de jeux ou... enfin c'est... c'est... (Hésitation)

Vous avez apprécié le côté interactif. Voire même peut-être ludique ?

$>$ Ludique voilà.

Autre chose?

$>$ (Hésitation) Non, non. J'ai apprécié l'interface. Ça m'a appris pas mal de choses, vu que mon diabète est récent, il y a des aspects de la maladie sur lesquels je ne m'étais pas encore penchée.

Quand votre diabète a-t-il été diagnostiqué ?

$>$ En février.

En février 2013 ?

$>$ Oui.

Vous me dites que vous avez appris des choses. Vous avez quelques exemples ?

$>$ Par exemple l'hémoglobine glyquée exactement euh... Parce ce que bon je n'en ai pas encore fait. Donc ça m'a un peu permis de comprendre comment ça fonctionnait, comment on établissait les résultats sur trois mois. Ça peut être des infos... c'est pas non plus très poussé mais je pense qu'on n'a pas besoin de comprendre plus, après c'est au niveau médical. Mais là déjà ça permet de bien aborder le sujet.

Avoir au moins une notion de ce que ça représente ?

$>$ Oui voilà, voilà. Représenter un petit peu. Ça et puis aussi oui quelque chose, au niveau de... Je ne pensais pas que ça pouvait être autant individualisé les résultats glycémiques. Par exemple selon... enfin pour moi c'était vraiment, il y avait une tranche à respecter ; c'était pour être bien entre 0.90 et 1.20 ; et en sortant de ça, ça n'allait plus. Mais c'est vrai que j'essayais parfois de trop respecter ça et je me trouvais beaucoup trop en hypo dès que je sortais. Là ça devenait ma hantise ces derniers temps de devoir me resucrer ou de m'asseoir en pleine rue et... Parce qu'en fait selon ce qu'on fait... c'est vrai que c'est pas grave si je vais courir et qu'avant d'aller courir je suis à 1.50 , c'est même mieux enfin, ça me permet de...

C'est ce que vous avez appris sur le site INDIGO ?

$>$ Oui voilà. De relativiser que bah oui je suis peut-être à 1.50 avant d'aller courir mais il vaut mieux.

Comment vous qualifieriez ce que ça vous a apporté ?

$>$ Bénéfique pour mon quotidien.

Vous dire que vous pouvez respecter une marge de glycémie un petit peu plus large pour vous sentir mieux dans votre quotidien ?

$>$ Voilà, oui.

D'accord. Y a-t-il autre chose que vous voulez me dire ?

$>\mathrm{Au}$ niveau de la glycémie capillaire j'avais tendance peut-être à la faire trop tôt après le repas donc ça m'a rappelé que c'est 2 à 3 heures. Souvent j'avais des résultats un peu trop élevés. Voilà juste ça et puis aussi de savoir qu'un non diabétique a des fluctuations aussi au niveau de sa glycémie. Parce que je me disais monter aussi haut enfin ça me faisait peur. De me dire le temps que l'insuline agisse au final je suis exposée au risque. Les fluctuations sont valables pour les non diabétiques en fait, le temps que le pancréas fasse son travail.

Tout à l'heure vous m'avez dit que vous aviez apprécié l'interface ? Vous pouvez préciser ?

$>\mathrm{Au}$ niveau du menu je trouve que c'est bien agencé, cette forme un peu circulaire. On peut... Enfin c'est clair et quand on rentre dans un chapitre il n'y a pas grand-chose à faire, c'est guidé. Il y a... Je ne sais pas, je trouve que ça fait un peu jeu vidéo. Il y a des moments où c'est quand même contrôlé, il $\mathrm{y}$ a des moments où il faut participer.

Vous avez apprécié le fait de devoir participer ?

$>$ Voilà, oui.

Le plan vous paraît-il logique ?

$>$ Oui. Pour moi les chapitres je ne vois pas dans quel autre ordre ils auraient pu apparaître. Non, je trouve que c'est logique.

Et au niveau de la navigation ? Vous n'avez pas eu de soucis particuliers ?

$>$ Non. A part quelques fois pour revenir au menu. 
Qu'est-ce qu'il se passait ?

$>$ Ça vient peut-être de ma connexion donc euh... (Silence)

C'était un peu long?

$>$ Oui voilà l'attente. Après je ne sais pas d'où ça vient.

Comment avez-vous visionné le site ? Racontez-moi.

$>$ J'ai commencé... Bah j'ai fait dans l'ordre, les deux premiers.

Vous n'avez pas vu le chapitre 3 ?

$>$ Non.

Vous n'aviez pas le temps ? Ou parce qu'il ne vous intéressait pas ?

$>$ La première fois c'était plutôt parce que ça ramait trop. J'avais une mauvaise connexion. Depuis j'ai changé de... de serveur. Mais après c'était plutôt manque de temps. Avec les horaires que j'ai faits je ne me suis pas repenchée dessus. Et puis là avant de vous rappeler j'ai voulu mais du coup j'ai reregardé que le $1^{\text {er }}$ et les autres vu qu'ils demandaient le code et que je ne m'en souvenais pas...

D'accord. Est-ce que vous irez le voir ultérieurement ce $3^{\text {ème }}$ chapitre ? Il vous intéresse ?

$>$ Oui, je pense oui. Oui parce que «Mes insulines » oui, je pense que... Oui, oui, j’irai le voir.

Donc vous m'avez dit que vous avez regardé le $1^{\text {er }}$ chapitre suivi du $2^{\text {ème }}$ chapitre ?

$>$ Oui.

La même fois ?

$>$ Non. J'ai regardé le lendemain.

Et le $2^{\text {ème }}$ vous l'avez regardé en entier et dans l'ordre ?

$>$ Non, le $2^{\text {ème }}$ je n'ai pas regardé dans l'ordre. Enfin je n'ai pas regardé dans l'ordre... j'ai moins participé que sur le $1^{\mathrm{er}}$.

C'est-à-dire ?

$>$ Euh par exemple... A un moment je me souviens... (Silence) Enfin il était moins visuel. J'avoue que j'ai moins accroché. Ça répétait certaines choses du $1^{\text {er, }}$ ça m'a moins apporté de choses.

Vous avez trouvé des redondances donc il vous a moins intéressée ?

$>$ Un petit peu oui.

D’accord. Donc vous avez accéléré des parties ?

$>\quad$ Il y a des parties que j'ai passées oui. Il y a des choses que je savais aussi, c'était pas forcément parce que c'était dans le $1^{\text {er }}$ chapitre mais parce que... voilà.

Pouvez-vous me donner un exemple de ce que vous avez passé ?

$>$ (Silence) J'essaie de me souvenir. Je sais qu'à un moment... J'ai passé deux témoignages je crois.

Avez-vous regardé le site seule ou avec quelqu'un ?

$>$ Je l'ai regardé seule.

On va parler du $1^{\mathrm{er}}$ chapitre : «Mes objectifs glycémiques ». Vous l'avez vu en entier ?

$>$ Oui.

Pouvez-vous me dire en une phrase de quoi il traite ?

$>$ Du diabète en général selon les personnes et leur mode de vie, selon ce que l'on peut rencontrer. Bah je me souviens de deux personnes : le jeune homme qui était dans mon lycée et aussi Manon, elle fait une femme enceinte et en fait c'est une amie à moi.

D'accord. C'est un hasard ou vous les avez rencontrés à l'hôpital ?

$>$ Ah non non non. Ben Manon elle n'est pas diabétique hein. Elle joue la femme enceinte avec le $\operatorname{Pr}$ BENHAMOU. Je ne savais pas du tout qu'ils étaient dans le site. C'est des amis de lycée quoi.

Pouvez-vous me dire ce que vous avez aimé dans ce chapitre ?

$>$ (Silence) J'ai aimé le fait qu'il y ait autant de participation et que l'on puisse à la fin avoir son... un récapitulatif de ses objectifs personnels de glycémies.

D’accord. Vous avez renseigné les différentes choses que l'on vous demandait?

$>$ Oui.

Et à la fin vous l'avez imprimé ?

$>$ Je n'ai pas d'imprimante mais je l'ai sur mon ordinateur. Je ne sais pas si ça va m'aider ou pas à respecter... parce que bon c'est quand même assez enfin euh... Voilà je l'ai rempli d'après ce que je pensais au jour d'aujourd'hui donc je ne sais pas si ça... Enfin ça m'aide déjà à voir que ça dépend de mon âge, ça dépend de mon quotidien euh...

D'accord. C'est vraiment ce qui vous a plu dans ce chapitre ? C'est de pouvoir définir finalement vos objectifs glycémiques avec une tranche plus large ?

$>$ Oui voilà. Oui.

Est-ce que vous pensez en discuter avec votre diabétologue ?

$>$ Je pense oui. Oui, oui, je pense qu'il faut en discuter parce que du coup il faut redéfinir vraiment les objectifs. Là jusqu'à présent je n'ai pas eu vraiment euh en fait de... vu que je n'ai pas encore fait 
d'hémoglobine glyquée on n'a pas encore parlé vraiment d'objectifs à atteindre, à respecter. Pour l'instant c'était vraiment euh... voilà je viens d'avoir la pompe.

Vous êtes sous pompe depuis quand ?

$>$ J'ai été hospitalisée le 1 juillet. Et on m'a mis la pompe à ce moment-là.

Y a-t-il d'autres choses qui vous ont plu dans ce chapitre ?

$>$ (Silence) Oui, les récapitulatifs à la fin d'une explication.

Les récapitulatifs ?

$>$ Les schémas. Avec le petit disque pour bien visualiser en fait, se rendre compte des glycémies ; là tout de suite je pense à l'explication: entre telle et telle glycémie éviter de conduire ou... Enfin voilà c'est bien euh...

Vous avez trouvé que le schéma était un bon récapitulatif, qu'il était bien conçu ?

$>$ Oui.

Autre chose ?

$>$ Non.

Y a-t-il des choses qui vous ont moins plu dans ce chapitre ?

$>$ (Silence) Non. Il n'y a rien de... de redondant (Silence) Non, tout est abordé.

Les informations contenues dans ce chapitre vous ont-elles permis de répondre aux questions que vous vous posiez sur vos objectifs glycémiques?

$>$ Oui, je pense oui.

Avez-vous des suggestions d'amélioration ?

$>$ (Silence) Non. Même la partie questionnaire où il faut cocher, il y a assez de choix pour ne pas être obligée de rentrer, enfin en tout cas pour ma part je n'ai pas eu à rentrer d'autres possibilités. Il y a «autres » à la fin. Donc non. Non.

On va parler du $2^{\text {ème }}$ chapitre : «Mes hypos, mes hypers ». Je vous en rappelle les 4 sous-chapitres : «Comment savoir où j'en suis au niveau de ma glycémie ? », « Comment je traite mes hypers et mes hypos? », «J'ai des problèmes d'hypos sévères » et «Je veux aller plus loin dans l'analyse de l'estimation de ma glycémie ». D'accord ?

$>$ Oui.

Vous m'avez dit que vous l'avez vu, pas forcément dans l'ordre et en passant un peu plus vite certaines choses que vous..

$>\quad \ldots$ que le $1^{\text {er }}$. Oui. J'ai passé plus de choses. Le $1^{\text {er }}$ je l'ai regardé vraiment en entier.

Est-ce que vous avez aussi rempli les différentes choses qu'il y a à remplir dans ce chapitre sur le ressenti des hypos et des hypers ?

> Euh non. Parce que justement... C'est peut-être parce que les hypers me font peur mais en fait ce chapitre, voilà je l'ai vraiment... il me paraissait... J'ai plus regardé ce qui était en rapport aux hypos pour pouvoir les éviter, j'ai plus regardé ça en fait. Je me souviens du $3^{\text {ème }}$ chapitre que j'ai regardé en entier, je crois que c'était le $3^{\text {ème. }}$

Avec la vidéo sur l'utilisation du Glucagon ?

$>$ Oui c'est ça.

Il vous a intéressée ce chapitre ?

$>$ Oui.

Il vous a plu ? Le fond, la forme ? Vous avez trouvé que c'était une bonne façon d'aborder les choses ?

> Oui. Je l'ai trouvé très... On ne m'avait pas vraiment expliqué déjà comment utiliser ce produit, dans quel cas vraiment, parce que ça m'arrive de faire des hypos et d'être vraiment très basse. Pour moi c'était vraiment quelque chose d'abstrait, que l'on n'avait pas forcément à avoir chez soi.

Vous pensez que c'est important de montrer cette séquence à votre entourage ?

$>$ Je pense que oui. J'aurais aimé que mon copain le regarde avec moi mais... Mais c'est vrai que je ne lui parle jamais de... ça reste quelque chose que je garde pour moi. On n'en discute pas vraiment. Ce n'est pas du tout présent dans... (Hésitation)

Ce n'est pas du tout présent dans votre quotidien de couple ?

$>$ Non. Voilà.

Vous aimeriez pouvoir en parler avec lui ?

$>$ Peut-être un petit peu mais je ne veux pas, non je ne veux pas que ça devienne euh... quelque chose euh de... enfin je ne veux pas que ce soit une part de moi trop présente, que ça devienne un côté... presque... un trait de caractère.

Malgré tout vous me dites que vous auriez aimé qu'il le voie. Cette séquence en particulier ou le site en général?

> Bah le site il était à côté de moi quand je me suis connectée et il m'a demandé ce que c'était. Mais il ne s'intéresse pas vraiment. Il dit que je gère bien, souvent il me dit: "Ah tu ne fais jamais d'hypos, tes résultats ont l'air bons ». Mais c'est vrai qu'il n'a aucune notion. Je pense que le site 
j'essayerai de lui montrer quand même parce que c'est des choses que je n'ai pas envie de lui expliquer. En fait je n'ai pas envie d'en parler vraiment. Mais s'il pouvait voir le site je pense que ce serait un bon... parce que ouais c'est clair.

Vous pensez à un moment donné lui montrer le site parce qu'il y a certaines choses...

$>\quad \ldots$ Qu'il faut qu'il sache oui.

On va revenir au chapitre 2. Qu'est-ce qui vous a plu dans ce chapitre au niveau du contenu ou de la forme?

$>$ A ce que je me souviens il y avait beaucoup plus de... enfin les témoignages étaient plus longs. Il y avait beaucoup plus de vidéos donc il y avait moins de... de participation que dans le $1^{\mathrm{er}}$.

Est-ce que ça vous a plu ou déplu ?

$>$ Je l'ai trouvé moins léger que le $1^{\mathrm{er}}$, c'est ce qui m'a poussée à avancer plus vite dans le chapitre. Mais je pense que je serais plus capable de le regarder aujourd'hui, j'étais peut-être un petit peu trop stressée la dernière fois. J'avais des mauvais résultats et ça me stressait... Je n'étais pas dans l'état d'esprit euh voilà... C'est peut-être aussi les gens dans le $2^{\text {ème }}$ chapitre, ouais c'était plus sérieux, c'était...

Ce n'était pas le moment pour vous de le regarder ? Vous pensez que vous y retournerez ?

$>$ Je pense que oui, qu'en même temps que le 3 je reregarderai le 2. Parce que j'ai raté peut-être plein de choses en passant trop vite.

Vous n'aviez pas envie d'entendre parler du diabète à ce moment-là ?

$>$ Oui voilà c'est ça.

Donc vous pensez qu'il y a quand même un moment propice pour regarder le site ? Ou en tout cas des moments qui ne le sont pas?

$>$ Oui complément. Déjà pas le soir comme je l'ai fait avec la fatigue et tout. J'étais en train de chercher du travail, je n'en trouvais pas. Ce n'était pas le moment.

D'accord. Vous pensez qu'il faut être dans un «bon état d'esprit » pour pouvoir recevoir toutes les informations, les témoignages des gens?

$>$ Voilà, en tout cas moi oui.

Vous m'avez parlé du côté qui vous a moins plu : qu'il y ait moins de côté ludique et participatif et que vous auriez peut-être aimé qu'il y en ait plus ?

$>$ Oui.

C'est une suggestion d'amélioration ? Comment aurait-on pu améliorer ça ?

$>$ (Hésitation) Plus euh... Enfin c'est vrai qu'il y avait déjà dans le $1^{\text {er }}$, mais ce côté dans le $1^{\text {er }}$ avec les schémas... Je ne sais pas plus de... Arriver à visualiser euh... Oui parce que c'est vrai qu'en vidéo on se rend compte, on voit les gens : «Oui effectivement j'ai eu la même expérience hier où je me suis sentie mal en voiture, j'ai été obligée de m'arrêter... » Enfin, je ne sais pas, moi en vidéo ça me parle moins parce que même si c'est la même situation je ne me reconnais pas vraiment dans la personne.

Les témoignages ce n'est pas quelque chose qui vous ait vraiment plu ?

$>$ Non, au niveau des hypos et des hypers c'étaient des situations... Peut-être le côté aussi que ça ne faisait pas vraiment vrai. Que ça fait acteur, je ne sais pas, peut-être.

Qu'est-ce qui vous a donné ce sentiment ?

$>$ C'est peut-être parce que je connaissais les gens dans la vidéo, je sais pas.

Vous pensez que ce ne sont pas des vraies personnes diabétiques ?

$>$ Oui, j'en suis sûre même. A part Jimmy mais sinon. Mais ça c'est un détail. Ce n'est pas quelque chose que je pense qu'il faudrait changer. Enfin c'était vraiment mon impression quand j'ai vu ce jour-là... C'était une impression mais ce n'est pas quelque chose que je pense qu'il faille changer, les vidéos sont claires et euh... Voilà c'est juste que ce jour-là je sais que j'ai passé les vidéos parce que... voilà.

Les témoignages dans ce chapitre ne vous ont pas vraiment parlé. Et dans le chapitre 1 vous les avez appréciés ?

$>$ J'ai plus apprécié dans le chapitre 1 parce que ça rentrait moins dans le vif du sujet. C'était encore des choses que j'arrivais à assimiler et contrôler. Enfin les informations... ça m'a moins stressée.

Y a-t-il autre chose que vous ayez apprécié dans le chapitre 2 ?

$>$ (Silence) Non.

Y a-t-il autre chose qui vous ait déplu ?

$>$ Non plus.

Est-ce que c'était ce que vous attendiez de ce chapitre ?

$>$ Oui je pense que je... Enfin voilà ça aborde les deux aspects assez clairement. Oui, non je ne vois pas. Après je connais pas encore assez pour dire : « Il manque telle chose... » 
Les informations contenues dans ce chapitre ont-elles répondu aux questions que vous vous posiez sur les hypos et les hypers?

$>$ En tout cas sur les hypos oui.

Moins sur les hypers?

$>$ Euh... oui, moins sur les hypers. Les hypos, oui c'est vrai que ça... ça m'a permis de savoir... enfin oui, voir comment je peux réagir autrement que comme je le faisais.

D’accord. Avez-vous des suggestions d'amélioration concernant ce chapitre ?

$>$ Oui. Peut-être glisser quelques récapitulatifs en plus comme il y avait dans le $1^{\mathrm{er}}$.

Sous forme de schémas?

$>$ Oui voilà peut-être un petit peu plus. Je pense que ça pourrait apporter un peu plus.

Le $3^{\text {ème }}$ chapitre vous ne l'avez pas vu et vous avez l'intention de le regarder ?

$>$ Avec le chapitre 2 oui.

Qu'est-ce que vous en attendez ?

$>$ Euh de faire moins d'erreurs. Parce que bon j'avoue que c'est très souvent que je me trompe parce que j'évalue mal. Ce que j'attends de ce chapitre c'est de plus arriver à... oui c'est de mieux évaluer ce que $j$ 'ingère et comment bien doser la quantité d'insuline.

Moins d'erreurs dans le dosage de votre insuline ?

Voilà. Oui.

Autre chose ?

$>$ Non.

Sur l'ensemble de ce que vous avez vu, quel est le chapitre ou le sous-chapitre qui vous a semblé le moins intéressant ou répondant le moins à des événements de votre vie quotidienne ?

$>$ Dans les deux premiers?

Oui, dans ce que vous avez vu. Ou que vous avez passé plus vite peut-être.

$>$ (Silence) Je sais que je n'ai pas regardé le début... Je crois que c'est le début du chapitre 2 parce que ça reprenait... comme j'avais regardé la vidéo la veille du 1er chapitre, je me souviens que le début... j'avais passé un peu directement le début du $2^{\text {ème }}$ chapitre parce que ça reprenait vraiment euh... (Hésitation)

Ce qu'il y avait dans « Mes objectifs glycémiques » ?

$>$ Oui.

Au contraire, quel est le chapitre ou le sous-chapitre qui vous a le plus intéressée ou qui vous semble le plus utile pour gérer votre diabète au quotidien ?

$>$ Ce qui m'a le plus intéressée c'était dans le chapitre 1. Après je ne me souviens plus comment il s'appelait mais c'était... Je ne me souviens plus à quel moment. Enfin chaque chose m'a apporté... Il y a un témoignage de la dame, quand elle dit que son objectif à elle c'est d'être dans les 1.40 par exemple pour ne pas être un poids en fait pour ses coéquipiers ou ses collègues. Je pense que c'est ça qui m'a le plus interpellée. C'était vraiment je pense quelque chose qui me pesais de vouloir trop être basse. C'est pour ça que je faisais hypos sur hypos parce que dans ma tête ce n'était pas comme ça qu'il fallait..

Et depuis vous faites moins d'hypoglycémies ? Est-ce que ça changé quelque chose dans votre façon de gérer votre diabète ?

$>$ Ah oui, oui complètement. Avant je faisais en moyenne, en moyenne parce que ça m'arrivait d'en faire plus ou moins, mais c'était deux hypos par jour.

Et ce n'est plus le cas ?

$>$ Là ça fait une semaine que j'en ai pas fait. Et maintenant ça m'arrive d'être plus vers 1.60 ou 1.70 mais je ne me fais pas d'injection, je sais qu'à ce moment-là je vais sortir et que ça va baisser tout seul et que je ne suis pas obligée de m'injecter à chaque fois... Je pense que j'avais trop recours à l'insuline et que ça va me permettre de voir que le corps peut réagir autrement à ça.

D'accord. Y a-t-il un thème qui n'est pas abordé dans le site INDIGO et qui vous a manqué ? Quelque chose que vous aimeriez trouver dans le futur?

$>$ Euh. Ce sera peut-être dans le chapitre sport enfin activités...

«Mes activités physiques ».

Oui voilà. Ils ne sont pas encore faits les deux derniers chapitres ?

Alors les deux derniers chapitres «Mon alimentation » et «Mes activités physiques » sont en cours d'élaboration. Vous souhaiteriez être tenue au courant quand ils seront disponibles ?

$>$ Oui je veux bien.

Ce sont des chapitres qui vous intéressent ?

$>$ Oui.

Peut-être plus « Mes activités physiques »?

$>$ Oui, mais aussi « Mon alimentation ». Je pense que ça peut être les deux plus intéressants au final. 
En dehors de ces deux thèmes-là vous pensez à autre chose ?

$>$ Je ne sais pas peut-être les voyages, ou ce qui est même juste excursions. Comment gérer quand on n'est pas dans un schéma habituel de vie, quand on n'est pas tous les soirs chez soi.

Il vous est déjà arrivé d'être en voyage ou en week-end et d'avoir des difficultés pour gérer votre diabète ?

Oui. Ne pas savoir vraiment... enfin par exemple dormir dans la voiture, de faire de la route, et d'être obligée de dormir sur une aire ou quoi, et de devoir changer mon cathéter. Je ne sais pas comment m'y prendre au niveau de par exemple de l'hygiène. Est-ce qu'il ne vaut mieux pas que j'aille me mettre ailleurs que dehors? Est-ce qu'il ne faut pas que je passe aux piqures en attendant de faire ça dans un endroit propre ? Enfin des choses comme ça qui sont peut-être simples mais...

Très bien. Autre chose ?

$>$ Non.

Sur une échelle de 0 à 10, quelle est votre satisfaction globale sur le site INDIGO : 0 vous n'êtes pas du tout satisfaite, 10 vous êtes très satisfaite ?

$>$ Je ne sais pas. 9. Enfin ce n'est même pas par rapport au site, c'est juste que je n'étais pas dans le même état d'esprit donc euh... Il m'a vraiment plu. Je sais que je regarderai les 2 et 3èmes chapitres et que quand ils sortiront les 4 et 5 aussi. Donc je mets 10. Je l'ai trouvé intéressant et je pense que des sites il ne doit pas y en avoir beaucoup sur Internet. C'est vrai que quand on tape « diabète » on tombe sur des forums, sur des... mais il n'y a pas beaucoup de sites comme ça interactifs et qui se penchent vraiment sur le diabète de type 1 en particulier.

Vous mettez 9 ou 10 ?

$>10$ (Rires).

Et donc vous estimez qu'INDIGO vous a apporté des éléments qui allaient vous aider dans la gestion de votre diabète au quotidien ?

$>$ Oui.

Donc essentiellement « Mes objectifs glycémiques ». Autre chose ?

$>$ Pour l'instant non mais par la suite oui ça va m'apporter d'autres choses, mais pour l'instant il n'y a que le 1 que j'ai vraiment apprécié.

Dans le questionnaire, à la question « Vous êtes vous senti déstabilisée dans la gestion de votre diabète au quotidien ? » vous avez répondu « Oui, plutôt d'accord ». Vous pouvez m'en dire plus ?

$>$ Moi ce qui me fait vraiment peur dans le diabète ce sont les complications. Du coup c'est les hypers qui me font vraiment peur. Et le $1^{\text {er }}$ chapitre m'a... enfin voilà ça m'a un peu permis de relativiser par rapport à ça. Que bon voilà, il y a des moments où on parle de résultats de plus de 2 grammes et ça a l'air presque humain. J'ai été déstabilisée mais de façon positive, ça m'a permis de prendre du recul, de voir ça autrement au quotidien, de relativiser certaines choses et d'être plus attentive sur d'autres.

Conseilleriez-vous le site INDIGO à une autre personne diabétique de type 1 ?

$>$ Oui.

A un membre de votre entourage ?

$>$ Aussi oui.

Donc à votre ami quand le moment sera venu entre guillemets. Vous pensez à quelqu'un d'autre ?

Non. Peut-être oui amis, proches, des gens qui seraient intéressés.

D'accord. A leur demande?

$>$ Voilà, c'est ça.

Avez-vous autre chose à me dire ?

$>$ Non.

Merci beaucoup. 


\section{Entretien n ${ }^{\circ} 10$ :}

Quelle serait la première chose que vous auriez envie de me dire après avoir exploré le site INDIGO ?

$>$ J'ai tout regardé le site. Bon j'ai vu qu'il y avait des choses qui n'étaient pas encore terminées hein comme l'alimentation, et c'est vrai que j'aimerais bien que ça soit terminé, enfin je pense que ça doit être vachement utile. J'avais fait moi un stage d'insulinothérapie il n'y a pas très longtemps, donc c'est vrai que ça m'a rappelé deux, trois choses qui me semblaient importantes et dont je ne me rappelais plus. Notamment pour tout ce qui est Glucagon tout ça, ça j'ai trouvé que c'était super parce que ça a vachement rassuré ma mère parce que eux ils avaient un petit peu peur par rapport à ça, déjà.

Alors ça veut dire que votre maman a vu le site aussi ?

$>$ Oui, bon elle n'a peut-être pas tout regardé mais en tout cas tout ce qui est... enfin ce qui concernait l'utilisation du Glucagon elle a regardé. Donc ça l'a rassurée parce que c'est vrai que bon pour l'entourage, c'est toujours un petit peu stressant quand même de....

... Ça l'a rassurée sur les gestes qu'elle devrait faire éventuellement ?

$>$ Oui, parce que c'est vrai que le fait d'expliquer comme ça à l'oral, bon, on se dit on comprend. Mais le fait de le voir c'est vrai que c'est beaucoup plus rassurant pour les gens et puis c'est mieux, même moi ça m'a rassurée quand même. Savoir comment on utilisait vraiment le produit, comment on l'injectait etc. Euh... c'est plus parlant je trouve de le voir en fait.

D'accord. Il y a d'autres personnes qui ont vu le site ou des parties du site ?

$>$ Oui il y a ma sœur, enfin oui ma sœur et ma maman l'ont vu.

Et votre sour vous a fait des retours? Elle l'a vu avec vous ou toute seule?

$>$ Euh elle l'a vu avec ma mère. Non mais elle m'a dit c'est vrai que pour elle c'était, c'était bien parce que au moins ils savent comment on l'utilise. Parce qu'elle avait été aussi en stage d'insulinothérapie; ils demandent si des membres de la famille veulent venir; pour qu'ils leur expliquent justement en cas de malaise comment procéder. Et c'est vrai qu'elle m'a dit que c'est vrai que le fait de le voir, bon ben, c'est... déjà c'est un peu dédramatisant parce que bon, on se dit c'est pas bien compliqué et puis c'est surtout eux ils ont peur de piquer en fait, ils ne savent pas trop où piquer et puis ils ont un peu peur par rapport à ça. Donc ça j'ai trouvé que c'était vraiment super.

Ils ont vu d'autres séquences que celle des hypoglycémies sévères ?

$>$ Euh oui, enfin ma maman elle a regardé, enfin euh, elle a parcouru un peu comme ça. Bon elle c'est vrai qu'elle est super angoissée depuis que j'ai le diabète, ça l'angoisse. Mais bon ils expliquent au départ c'est vrai qu'il faut quand même gérer tous les jours, bon, ils le savent hein mais du coup c'est vrai que elle, de savoir que je dois toujours prévoir je pense que bon ça l'a un peu... Mais c'est vrai que ça m'angoisse. Mais elle elle dit que c'est bien de le savoir et elle me dit c'est vrai que mieux... plus tu le gères correctement et moins tu as de risques d'avoir de problèmes donc euh...

Et votre maman vous a fait un retour global sur le site ?

$>$ Elle elle a trouvé que c'était parlant, que vraiment c'était euh... Non elle a bien aimé le site, elle a dit vraiment... elle a gardé les codes. (Rires) Elle a dit : « Je garde ».

Votre maman et votre sœur c'était une demande de leur part ? Ou c'est vous qui...

$>$... Non, bon c'est vrai que j'étais allée d'abord chez elle pour essayer de regarder le site et en fait non, ma mère elle l'a tout de suite regardé sans que je lui demande quoi que ce soit. Non non je pense qu'elles sont vraiment intéressées, pour mieux comprendre aussi comment ça fonctionne hein je pense.

Très bien. Alors est-ce que vous avez...

$>$... Par contre si je peux, moi ce que j'ai vraiment bien aimé c'est aussi les documents qu'on peut imprimer, le fait qu'on puisse imprimer les démarches, le carnet INDIGO par exemple. On ne nous en avait pas parlé je trouve ça super aussi parce que c'est vrai que j'ai essayé de le faire justement, par rapport aux hypos et aux hypers, les sensations qu'on pouvait ressentir, et c'est vrai que le fait de vraiment essayer d'analyser ce qu'on ressent je trouve qu'on est plus à l'écoute et du coup c'est plus... c'est plus fiable en fait. On a plus l'impression de savoir ce qu'on ressent. Parce que moi par exemple j'avais des hypos que je ne ressentais pas, j'avais un lecteur qu'était pas... qu'était pas bon, on l'avait testé à l'hôpital justement quand j'y étais allée, et il s'est avéré qu'il était faux en fait, donc j'étais beaucoup plus basse et du coup je pense que je m'étais habituée en fait à être en hypo. Et du coup des fois je disais : «A 0,80 moi je me sens pas bien » et j'avais dit à la diabéto : « Moi je ne me sens pas bien à 0,80 alors que je ne suis pas encore en hypo » et... et je lui ai dit : «Je ne sais pas, j'ai l'impression que je commence à être pas bien » et puis finalement après avec le temps je pense que je me suis habituée. Et du coup, de mieux analyser, ça me permet de me sentir tout de suite plus en alerte. Dès que je commence à sentir que j'ai un doute, bon ben, je me pique hein.

Vous avez l'impression que le fait d'utiliser le carnet INDIGO a affiné vos ressentis ? 
$>$ Ouais, ouais je pense ouais. Moi pour moi ça m'aidera je pense. Alors je ne le ferai pas tout le temps. Tout le temps, peut être pas non plus. Mais c'est vrai que le faire peut être une fois par mois ou... Même là je vais avoir un rendez-vous avec ma diabéto, je pense que je lui en parlerai aussi.

D'accord. Donc vous pensez que vous allez reparler avec elle de certains éléments que vous avez vus sur le site?

$>$ Euh oui oui, je pense que je vais en reparler. Ben déjà parce que mon hémoglobine glyquée à moi pour l'instant euh ; moi ça fait que neuf mois que j'ai du diabète ; mais jusqu'à maintenant je tournais à 4 alors euh... pour l'instant...

Votre diabète a été diagnostiqué quand ?

$>\quad$ Il a été diagnostiqué le 28 décembre.

D'accord. Vous avez imprimé d'autres choses sur le site INDIGO ?

$>$ Alors la seule chose sur le carnet INDIGO, ce que je trouve qui est un petit peu dommage, c'est qu'il n'est pas au format A4 donc il est petit en fait quand on l'imprime. Et les gens qui n'ont pas de photocopieur je pense que pour l'ajuster, enfin moi je l'ai imprimé comme ça hein, c'est vrai que j'ai dû faire un bric-à-brac pour l'agrandir en fait.

Donc ça pourrait être une suggestion d'amélioration alors ? Mettre le carnet INDIGO en format A4 ?

$>$ Ouais. Le truc que j'ai, enfin pas moins aimé, mais c'est vrai qu'il y a un temps d'attente en fait à chaque fois euh, vous savez, il y a le loading qui se met en route comme ça. Moi des fois entre chaque plan, c'était un petit peu long.

Alors entre chaque diapositive ? Entre chaque sous-chapitre ?

$>$ Euh bah des fois c'était à chaque fois que je passais une flèche, vous savez on passe les flèches, des fois c'était un petit peu long. Bon après c'est le seul bémol que j'ai trouvé.

Vous avez utilisé quel navigateur?

$>$ Euh Chrome.

D'accord. Et est-ce que ça vous arrive de temps en temps d'avoir des choses qui mettent un peu de temps à charger ou...

$>$... Oui non mais je pense que c'est peut-être plus lié à mon ordinateur mais c'est vrai que du coup, bon ben, c'était un peu long quoi. Sinon qu'est-ce que j'avais vu qui était bien, que j'avais bien aimé... Le seul truc peut-être moi qui manquait, c'est peut-être une suggestion, c'est peut-être si on fait des erreurs de piqûre, le fait de prendre de la rapide par exemple au lieu de prendre de la lantus par exemple. Comment réagir ? Ça euh... Peut être que... enfin bon c'était pas... mais bon c'est vrai que des fois on se pose plein de questions.

Est-ce que ça vous est déjà arrivé ?

$>$ Non mais ça a failli m'arriver au tout début, c'est pour ça je me suis dit : « Mince, qu'est-ce que je fais si un jour ça m'arrive, quoi », je savais pas trop.

D'accord.

$>$ Qu'est-ce que j'avais trouvé qui était bien aussi, que je voulais imprimer mais je n'ai pas eu le temps de le faire encore parce que je ne l'ai pas fait tout de suite, imprimer, donc après je ne savais plus ou c'était à vrai dire.

Alors dans la suite logique du carnet INDIGO il y a la grille d'analyse des erreurs.

$>$ Alors je l'ai trouvée bien mais moins parlante pour moi, ça me parle moins.

D'accord donc vous ne pensez pas l'utiliser ?

$>$ Je pense que je ne l'utiliserai pas non. Parce que je la trouve un petit peu compliquée à utiliser, enfin c'est pas compliqué mais ça me parle moins, le graphique comme ça, ça me... Je pense que je l'utiliserai pas ça.

D'accord.

$>$ Par contre moi ce que je voulais utiliser, je ne sais plus dans quel chapitre c'est, je crois que c'est dans les insulines, c'est quand on est malade, ah oui, quand on a de l'acétone. Ça j'ai trouvé que c'était bien parce que c'était un rappel de ce que j'avais vu en insulinothérapie. Mais je n'ai pas retrouvé... alors par contre je ne sais pas... alors ça c'est vrai que je n'ai pas eu le temps de rerechercher à vrai dire parce que j'ai visionné tout le site et après j'ai voulu imprimer les documents parce que chez moi j'ai pas d'imprimante. Et c'est vrai que quand je suis retournée au boulot j'ai dit : «Ben tiens entre midi et deux des fois je vais avoir le temps de le faire, je vais regarder » et après je ne savais plus où c'était à vrai dire. Et je me demandais, il me semblait que j'avais vu tout un, à un moment un sommaire, mais je ne sais pas si on arrive à retrouver les documents à imprimer.

Le sommaire... le plan général vous voulez dire ?

$>$ Oui mais est-ce que les documents à imprimer sont signalés à un moment dans le plan général ou pas?

Pas dans la première page. Mais ils sont rappelés à la fin des chapitres. La dernière diapositive de chaque chapitre ou sous-chapitre. 
Mais donc ça veut dire qu'il faut qu'on refasse tout le chapitre pour imprimer les documents.

Alors sous la vidéo il y a plusieurs petits onglets dont un onglet « Diapositives », et on peut les passer comme ça et arriver directement à la dernière.

$>$ Oui mais alors du coup comme je ne savais plus dans quel chapitre c'était..

Oui, effectivement. Est-ce que vous trouvez que le plan du site est logique ? Est-ce que vous trouvez que le site est bien structuré ?

$>$ Oui moi j'ai trouvé que c'était bien fait, oui.

Je voudrais juste vous poser pendant que j'y pense une question sur la grille d'analyse des erreurs. Le schéma, l'explication, est-ce que c'était clair ?

$>$ Euh alors clair oui et non. Enfin j'ai compris le fonctionnement général. Je ne suis pas sûre que j'arriverai à l'utiliser toute seule.

D'accord donc c'est ça qui vous plait moins ? Ça vous paraît un petit peu difficile ?

$>$ Ouais et puis moi le graphique, je pense que c'est vraiment une question de personnalité, ça ne me parle pas.

Est-ce que vous pensez qu'on aurait pu l'améliorer ? Vous pensez à un moyen de rendre la chose plus simple ou plus attrayante?

$>$ Ben après je pense que c'est vraiment lié à la personne. Il y a des gens pour qui le graphique va leur parler, moi je vais plutôt être... Non je pense que c'est vraiment une question de personnalité, moi je sais que le graphique, même amélioré, je pense que je ne l'utiliserai pas.

D'accord. Est-ce que vous avez autre chose à me dire ?

$>$ Euh. Bah est-ce que vous avez terminé l'alimentation, le sport etc. ?

Ce sont des chapitres qui vous intéressent « Mon alimentation », « Mes activités physiques »?

$>$ Oui.

Ils sont encore en préparation. A ma connaissance le chapitre «Mon alimentation» devrait être disponible au mois d'octobre. Est-ce que vous désirez être informée lorsqu'ils seront disponibles ?

$>$ Ah oui, oui. J'aimerais vraiment que l'on m'informe.

D'accord. Je vais vous demander comment vous avez visionné le site INDIGO ?

$>$ Oh ben j'ai commencé par le début hein. J'ai commencé par les hypos euh... Après j'ai euh...

Alors le tout premier chapitre c'est « Mes objectifs glycémiques ».

$>$ Voilà. Oui mais je pense que j'ai suivi l'ordre.

L'ordre des chapitres. Et à l'intérieur des chapitres vous avez tout regardé en entier et dans l'ordre proposé ?

$>$ Oui.

Et est-ce que vous avez fait un seul visionnage ou est-ce que vous l'avez vu en plusieurs fois ?

$>$ Alors euh qu'est-ce que j'ai fait? Non j'ai fait, oui j'ai fait tout en une soirée. Non j'ai tout fait.

Les trois chapitres d'un coup, d'accord. Et ça vous a paru...

$>$... Long. Je l'ai trouvé long parce que c'est vrai que tout regarder d'un coup, c'était long.

Ça fait beaucoup d'informations ?

$>$ Ça fait beaucoup d'informations.

Vous pensez qu'il y a des informations que vous avez pu éventuellement « perdre » entre guillemets en le faisant...

$>$... Ah oui, moi il faut que je le regarde, de toute façon je le re-regarderai pour mon info personnelle, je vais le re-regarder oui.

D'accord. En entier ou vous avez ciblé certaines choses que vous...

$>$...Euh ben je pense que ce serait bien que je le re-regarde encore une fois en entier parce que justement s'il y a des choses qui me paraissent importantes, ben de noter, comme ça je sais où je peux les trouver dans les chapitres. Voilà quoi.

D'accord. Est-ce que vous avez encore quelque chose à me dire spontanément ?

$>$ Euh peut... euh... alors... Moi perso, si il y a peut-être juste un truc que j'ai moins aimé ; je me suis posé la question en fait quand j'ai visualisé le site ; c'était la voix de la personne qui parle en fait. Alors j'ai demandé à mon fils parce que j'ai dit c'est peut-être moi qui accroche moins bien, bon en même temps, lui il m'a dit : «Elle est pourrie la voix ». (Rires)

Alors elle est « pourrie » : elle ne vous plaît pas à l'oreille ou le son est mal audible ?

$>$ Non non le son était bien. Mais... bon enfin c'était rien de bien particulier mais je me disais si il y a un bémol peut être que c'était ça aussi, la voix était pas...

Pas agréable ?

> Pas désagréable non plus, mais bon, j'ai demandé à mon fils exprès, il a 12 ans donc je lui ai dit : «Bon qu'est-ce que t'en penses ? », il m'a dit : «Non...»C'était un peu... je sais pas, je sais pas, je ne sais pas comment l'expliquer mais la voix c'était moins, moins...

La voix masculine ou la voix féminine ? Enfin une des voix parce qu'il y en a plusieurs ? 
Féminine.

D'accord. Et les autres voix ? Il y en a une en particulier qui vous a moins plus ?

$>$ Non mais ce n'était pas forcément les personnages quand ils parlaient etc.

Oui, oui. Les voix off ?

$>$ Oui.

D'accord. Autre chose ?

$>$ Non, non. Je crois que non.

Alors si vous voulez bien on va parler du 1er chapitre : « Mes objectifs glycémiques ». Est-ce que vous pouvez me dire en une phrase de quoi parle ce chapitre ?

$>$ Euh c'est les objectifs qu'on se fixe, euh... pour être bien en fait, pour... pour faire nos activités de tous les jours quoi, enfin moi je l'ai compris comme ça.

Est-ce que vous pouvez me dire ce que vous avez apprécié dans ce chapitre au niveau du contenu ou au niveau de la forme?

$>$ Alors attendez il faut que je me rappelle. Alors ils nous posent des questions dans ce chapitre je crois... (Silence) Alors du coup je l'ai regardé la semaine dernière, je m'en rappelle. Euh... (Silence) Oui il y a des personnages qui parlent hein, c'est ça ? Alors ça j'ai trouvé que c'était pas mal aussi parce que bon c'est vrai qu'on s'identifie aussi aux gens de la vie de tous les jours. J'ai trouvé que ça c'était pas mal.

Vous avez pu vous identifier facilement aux personnages?

$>$ Oui et puis ce que j'ai trouvé bien c'est que tout le monde n'a pas la même manière de percevoir sa façon de vivre et sa... enfin sa moyenne, enfin là où il veut se situer. C'est vrai que ça par contre ça m'a un peu... Je me suis dit bah oui, effectivement, on n'est pas obligé d'être à 0,80 ou à 1,20 pour être toujours super bien. Peut-être que c'était ça dont peut-être je voulais parler justement avec ma diabéto, je sais plus.

Revoir les objectifs glycémiques, vos propres objectifs glycémiques ?

$>$ Peut-être. C'est vrai que c'était quelque chose que je m'étais dit que je reverrais justement avec ma diabéto pour ça oui.

Essentiellement ce qui vous a plu donc ce sont les témoignages ?

$>$ Les témoignages j'ai bien aimé oui.

Vous vous souvenez d'autre chose qui vous ait plu ?

$>$ Euh, j'ai tellement fait tout en même temps que du coup euh... Qu'est-ce qu'ils disaient? Ils montraient les plages, ils nous expliquaient là où on est bien ; de 0,80 à 1,20 hein je crois que c'est ça; après... 2 heures après le repas... bon ça je le savais déjà, donc c'était pas... Mais bon, non je n'avais rien de particulier là-dessus.

Est-ce que vous vous souvenez de choses qui vous ont moins plu dans ce chapitre ?

$>$ Bah je peux pas dire qu'il y ait vraiment quelque chose qui ne m'ait pas plu dans le site hein.

Est-ce que les informations contenues dans ce chapitre ont répondu aux questions que vous vous posiez sur vos objectifs glycémiques?

$>$ Euh oui. Ça j'ai trouvé que c'était pas mal, oui.

Est-ce que vous avez des suggestions d'amélioration pour ce chapitre ?

Bah non et puis je n'ai peut-être pas encore assez de recul, mais non j'ai trouvé que c'était complet.

D'accord. On va parler du $2^{\text {eme }}$ chapitre : «Mes hypos, mes hypers ». Je vais vous en rappeler les 4 sous-chapitres : «Comment savoir où j’en suis au niveau de ma glycémie ? », «Comment je traite mes hypers et mes hypos ? », «J'ai des problèmes d'hypos sévères » et « Je veux aller plus loin dans l'analyse de l'estimation de ma glycémie ».

$>$ Oui voilà.

Vous m'avez dit l'avoir vu en entier, dans l'ordre défini.

$>$ Oui.

Vous l'avez vu avec votre maman et puis ensuite votre maman l'a revu avec votre sœur mais sans vous c'est ça ?

$>$ Non. Elle a essayé d'installer l'ordi. Au début on n'arrivait pas à le visionner en fait. Parce que justement on avait les voix qui buggaient en fait chez elle.

Chez votre maman aussi ?

$>$ Oui. Chez ma maman aussi oui. Donc ça buggait tout le temps. En fait... bon je suis partie de chez elle en lui disant : «Ecoute je vais essayer de chez moi, peut-être que ça fonctionnera mieux » etc.

Vous avez compris d'où venait le problème?

$>$ Non, bah je sais pas. Dès qu'on l'a mis on entendait..., alors en fait on voyait le personnage mais les voix buggaient donc on n'arrivait pas à suivre donc ça s'arrêtait donc il y avait le loading qui se mettait en route et on n'arrivait pas à... Alors ça nous l'a fait au début et puis alors bon ça a bien duré $1 / 4 \mathrm{~d}$ 'heure hein et puis après d'un coup elle a pu visionner la totalité en fait. 
Vous aussi il me semble que vous aviez eu un problème au départ pour visionner le site ?

$>$ Oui mais moi je n'avais pas utilisé le bon navigateur, je n'avais pas... bon donc euh...

Est-ce que ça vous a gênée le fait que ça ne soit pas disponible sur tous les navigateurs ?

$>$ Euh... Bah gênée oui et non. Enfin, non, non ça ne m'a pas gênée plus que ça. C'est vrai que sur le coup j'ai essayé Internet explorer, je pense que j'avais mis. C'est vrai que si vous ne m'aviez pas appelée je ne suis pas sûre que j'aurais été plus loin.

Et finalement vous avez téléchargé Google Chrome. Et ça s’est fait facilement ?

$>$ Oui, oui, oui, ça s'est fait facilement.

Alors. Est-ce que vous pouvez me dire en une phrase de quoi traite ce chapitre ?

$>$ Comment gérer ses hypos, ce qu'on ressent par rapport à ses hypos, enfin notre ressenti personnel en hypo et en hyper et puis comment on gère le resucrage. Enfin moi j'ai compris comme ça.

Qu'avez-vous aimé dans ce chapitre au niveau du contenu et de la forme ?

$>$ Alors là il y avait des questions je crois. Comment on ressentait nos hypos. Alors ça j'ai trouvé que c'était pas mal aussi. Parce que justement ça permet d'analyser un peu comment on se sent. On ne sait pas toujours comment on se sent...

... Alors vous l'avez fait ? Vous avez coché vos propres ressentis ?

$>$ Oui, oui, oui, j'ai coché. Mais c'est vrai qu'il y a aussi des moments où les hypers et les hypos ça peut se ressembler. Donc je pense que... C'est pour ça qu'après affiner sa recherche j'ai trouvé que c'était pas mal et pour moi le carnet INDIGO je trouve que c'était pas mal aussi. Parce que de bien noter finalement les choses qu'on ressent je trouve que ça nous aide à mieux comprendre les choses aussi j'ai trouvé.

Ça vous aide à mieux comprendre votre glycémie ?

$>$ Oui.

Y a-t-il autre chose que vous avez aimé ?

$>$ (Silence) Le fait que l'on revienne dessus parce qu'ils nous rappellent ce qu'on a mis en fait, ce qu'on a coché justement donc euh...

Vous l'avez imprimé ça ?

$>$ Non justement parce que la première fois je n'ai fait que visionner et ça c'est vrai que c'est le... Je n'ai pas d'imprimante chez moi donc je ne pouvais pas l'imprimer donc c'est vrai qu'il fallait que je le fasse du travail et du coup je ne l'ai pas fait. Mais ça par contre c'est vrai que pour ça je pense que c'est un point positif parce que pour pouvoir discuter même avec sa diabéto ; parce que moi je ne la vois qu'une fois... bon là je ne l'ai pas vue en plus...; mais c'est vrai qu'après on oublie en fait les choses qu'on doit leur demander...

Donc ça c'est une chose dont vous auriez envie de discuter avec elle ?

$>$ Ouais.

Y a-t-il autre chose qui vous vienne à l'esprit ?

$>$ Euh... Qu'est-ce qu'il y avait d'autre après, je ne me rappelle plus. Non alors dans ce chapitre après il y avait le carnet que j'ai trouvé bien. Oui c'est le carnet que j'ai trouvé vraiment le plus, le plus dans ce chapitre. C'est vraiment ça que j'ai bien aimé.

Et puis la séance « J'ai des problèmes d'hypos sévères » ?

$>$ Voilà oui.

Est-ce qu'il y a des choses qui vous ont moins plu ?

$>$ Alors moi ce chapitre il m'a vraiment plu parce que c'est vrai que le fait de... Dans celui-ci je crois qu'il y avait le Glucagon aussi hein dedans?

Oui, oui, c'est ça. « J'ai des problèmes d'hypos sévères ».

$>$ Donc ça j'ai trouvé qu'elle était vraiment particulièrement bien la séance avec les personnes qui expliquaient tout. J'ai trouvé que c'était bien et pour mon entourage et pour moi. Le carnet c'est vrai que j'ai trouvé que c'était super aussi. Euh... Et puis le fait de poser des questions comme ça, c'est interactif, je trouve que c'est... On ne s'ennuie pas quand on regarde le site. Parce que c'est vrai que ça répond à beaucoup de questions.

Vous l'avez trouvé interactif ?

$>$ Oui, j'ai trouvé que c'était pas mal. Et je ne sais plus si c'est dans ce chapitre-là où ils parlent justement que la température du corps, la chaleur etc. Ça peut faire varier aussi la glycémie.

C'est dans « Mes insulines ».

$>$ Ah c'était dans « Mes insulines ». Je suis hors-sujet. Mais c'était pas mal.

Ça aussi c'est une information qui vous a...

$>\quad \ldots$ Bah oui parce que moi par exemple quand je me douche, si je mets de l'eau chaude, d'un seul coup je peux vite me retrouver en hypo alors que je viens de manger il y a moins d'une heure.

Et avant vous ne compreniez pas pourquoi ? 
$>$ Oui, je me disais «Mais pourquoi ? », après je me disais : «Est-ce que je ne me suis pas fait la bonne dose d'insuline? » alors que je pèse tous mes aliments et tout donc je fais super attention quoi.

D’accord. Donc ça vous a donné des éléments de...

$>\quad \ldots$ Bah je pense que le fait de plus on connait, moins on est stressé. Parce qu'on se dit finalement peut-être que voilà, ça a une incidence. Si on sait qu'on a mangé peut-être plus léger j'en sais rien, peut-être qu'on préfêre se doucher le lendemain, enfin j'en sais rien parce que moi le matin je ne prends pas d'insuline par exemple. Ça me permet de mieux comprendre et je pense que le fait de mieux comprendre... Enfin de mettre des mots sur ses sensations c'est hyper important je pense, on doit dédramatiser aussi parce qu'on comprend mieux. Enfin moi je sais que je fonctionne comme ça après les autres je sais pas, mais moi perso je fonctionne comme ça et je trouve que c'était intéressant à ce niveau-là ouais.

D'accord. Est-ce que les informations contenues dans ce chapitre ont répondu à toutes les questions que vous vous posiez sur vos hypos et vos hyperglycémies?

$>$ Bah ouais, je pense qu'il était vraiment bien celui-ci, ouais je l'ai trouvé vraiment bien.

Avez-vous des suggestions d'amélioration ?

$>$ Non, pas sur celui-ci. Franchement non.

Alors on va passer au $3^{\text {ème }}$ chapitre : «Mes insulines ». Je vais vous le redétailler. Les sous-chapitres étaient : «Comprendre mon traitement par insuline », «Mes injections d'insuline », « Comment définir mes doses d'insuline ? » et « Le manque d'insuline : hyperglycémie et acétone ». D'accord ?

$>$ Oui.

Vous m'avez dit l'avoir vu aussi en entier et dans l'ordre ?

$>$ Oui.

Pouvez-vous me dire en une phrase de quoi traite ce chapitre ?

$>$ Bah là ils ont présenté les stylos et l'insuline, comment elle fonctionne dans notre corps en fait. Et puis qu'est-ce que... qu'est-ce que je pourrais dire d'autre... Là je l'ai mal résumé. (Rires) Je ne suis pas contente de moi. Euh... Oui enfin c'est comment adapter sa dose d'insuline par rapport à son alimentation euh... enfin ouais comme ça ouais, par rapport à son alimentation ouais.

Pouvez-vous me dire ce qui vous a plu dans le contenu ou dans la forme ?

$>$ Alors celui-ci je l'ai trouvé euh... les stylos tout ça bon je m'en foutais un petit peu. Ils ont fait la présentation des stylos : la Lantus, la Novorapid. Ils ont parlé d'un à action intermédiaire que je ne connaissais pas par contre ça. Bon la pompe je suis pas concernée donc voilà

Alors qu'est-ce qui ne vous a pas intéressée dans la présentation des insulines ?

$>$ Alors. J'ai trouvé que c'était sans plus. Là où j'ai bien aimé dans l'insuline, ce qu'ils ont présenté par contre c'est le... ils ont expliqué le fait que l'insuline elle est très active je crois de 30 à 70 minutes. Elle est... c'est là... elle a un pic d'action qui est plus important et puis après ça redescend. Ça j'ai trouvé que c'était bien par contre.

Donc la cinétique des insulines ?

$>$ Oui. Par contre la seule chose que je n'ai pas comprise justement j'allais demander peut-être à ma diabéto c'est par exemple elle monte effectivement jusqu'à admettons 70 minutes. Quand elle redescend ça veut dire que notre glycémie elle remonte ou elle ne remonte pas à ce moment-là. Ça...

... Ça c'est une question que...

$>\quad \ldots$ Que je me suis posée.

A la suite du...

$>$... Oui. Je ne savais pas en fait. Je me suis dit... Parce que c'est vrai que des fois elle agit mais estce que ça veut dire qu'après notre glycémie elle va... enfin je sais que ça a une action normalement de 3 heures l'insuline donc euh ça couvre 3 heures mais après, ça, je n'ai pas compris ça. Alors j'ai peut-être mal regardé. Je me suis dit peut-être qu'il faudrait que je re-regarde une autre fois pour peut-être mieux comprendre.

D'accord. Donc ça n'a pas répondu finalement à toutes les questions que vous vous posiez sur les insulines ou même ça vous en a posé d'autres?

$>$ Ça m'en a posé une surtout alors peut-être pas d'autres. Mais celle-ci du coup je me la suis posée quand j'ai vu euh... Mais bon c'était intéressant du coup mais...

Donc la cinétique des différentes insulines ça vous a intéressée. Vous vous souvenez quel moment vous a moins intéressée exactement ?

$>$ Quand ils parlent de stylos euh...

Sur la façon de se faire les injections vous voulez dire?

$>$ Non, non. Pas forcément. Ils nous présentent les stylos vous savez à un moment donné. Je sais plus qu'est-ce qu'ils nous parlent de quoi. Qu'est-ce qu'ils nous parlent ? Je... Il y a un truc... Bon j'ai 
trouvé... Peut-être parce que le $2^{\text {ème }}$ chapitre ça m'avait beaucoup plus intéressée aussi alors du coup le $3^{\text {ème }}$ je l'ai trouvé moins... Peut-être aussi hein, je ne sais pas.

D'accord. Est-ce qu'il y a d'autres choses qui vous ont plu ?

$>$ Alors oui, par contre là où j'ai bien aimé c'était au niveau de l'acétone, comment réagir par rapport euh quand on a de l'acétone parce que c'est vrai que bon ça m'est arrivée d'être en hyper et l'acétone je l'ai pas fait.

Vous ne l'avez pas recherchée?

$>$ La bandelette urinaire je l'ai pas cherchée, je l'ai pas faite. Alors par contre moi le problème que je rencontre par exemple avec justement l'acétone, peut-être que ça on aurait dû l'expliquer, moi je comprends rien comment il faut lire les bandelettes urinaires par exemple. Et je sais que quand j'étais en insulinothérapie j'étais pas la seule à ne pas savoir faire.

Et vous avez pu poser la question ?

$>\quad$ Ah bah non justement j'attends de voir ma diabéto pour lui poser la question parce que du coup bah je...

Donc une suggestion d'amélioration ça serait que l'on montre une bandelette et que l'on explique...

$>$... Peut-être ouais parce que ça du coup c'est resté en suspens pour moi et c'est vrai que je ne sais pas l'utiliser. Bon alors je suis rarement en hyper hein, tant mieux, mais... ça m'est déjà arrivée quand j'étais un petit peu malade ; et j'étais juste un petit peu malade ; alors je me dis là que l'on va attaquer l'hiver euh...

D'accord. Donc que l'on explique plus précisément comment se servir d'une bandelette et comment la lire ?

$>$ Bah oui, enfin c'est peut-être con mais... (Rires) Moi c'est vrai que je sais pas l'utiliser, j'ai essayé de faire une fois et je n'ai rien compris.

Autre chose ?

$>$ Euh... (Silence) Non je voulais dire un truc, je ne m'en rappelle plus. J'étais en train de penser à autre chose euh..

Allez-y prenez votre temps.

$>$ (Silence) Non, bah écoutez, je crois que ça ne va pas revenir.

Vous ne vous souvenez pas d'autre chose qui vous aurait particulièrement plu ?

$>$ Alors attendez. Qu'est-ce qu'il y avait dans ce chapitre? Bah si le fait d'imprimer aussi les documents ça j'ai trouvé que c'était bien parce qu'à ce moment-là on peut imprimer, là je trouve ça super mais bon je n'ai pas eu le temps de les imprimer donc euh... Qu'est-ce qu'il y avait d'autre... (Silence)

Si ça vous revient vous n'hésitez pas à me le dire. Est-ce qu'il y a des choses qui vous ont moins plu ?

$>$ Alors dans ce chapitre-là je crois qu'il n'y avait pas les mêmes personnages. Il y a une jeune je crois et une dame un peu... une autre. Je l'ai trouvée moins bien celle-ci je ne sais pas pourquoi. Alors pourquoi ? C'est vraiment le visuel hein, c'est pas euh...

D’accord. Vous avez moins réussi à vous identifier ?

$>$ Peut-être. Oui, oui.

Vous ne sauriez pas expliquer pourquoi ?

$>\quad$ Non je ne sais pas, une question de feeling. Je préférais vous le dire hein.

Ce n'est pas ce qu'elle disait en tout cas, ce ne sont pas ses propos ?

$>$ Non, non, mais c'est vrai que du coup ça m'empêchait un peu d'accrocher.

Bon, d'accord. Alors est-ce que les informations contenues dans le chapitre ont répondu aux questions que vous vous posiez sur les insulines?

$>$ Ah oui, par contre, je l'ai trouvé aussi... Je l'ai trouvé bien de toute façon. Oui.

Avez-vous des suggestions d'amélioration ?

$>$ Euh... Non. Bah je vous dis à part le... ce que je vous ai demandé là pour euh... voilà. Sinon non. Peut-être le seul truc c'est ce que je vous avais dit au départ si on se trompe peut-être d'insuline peut-être enfin, c'est vrai c'est assez bête, mais euh... ou alors peut-être le mettre dans le quiz parce que le quiz aussi je crois qu'il n'est pas fait non plus?

Non le quiz n'est pas encore accessible. C'est quelque chose que vous attendez aussi ?

$>$ Ouais ça moi j'aimerais bien ouais.

Très bien. Alors on va reprendre l'ensemble des chapitres et vous allez me dire quel est le chapitre ou le sous-chapitre qui vous a semblé le moins intéressant ou répondant le moins à des événements de votre vie quotidienne?

$>$ Oui alors ça c'est compliqué. Ils étaient tous intéressants et puis bon on est concernés par tous les chapitres dans notre vie quotidienne malgré tout. Non, moi j'ai trouvé qu'ils étaient...

... Aucun ?

$>$ Non. Moi pour moi ils étaient tous intéressants. 
D'accord. Quel est le chapitre ou le sous-chapitre qui au contraire vous a le plus intéressée ou qui vous semble le plus utile pour gérer votre diabète au quotidien ?

$>$ Bah moi c'était le 2 .

Le 2. Dans sa globalité ?

$>$ Et puis même dans la forme et tout je l'ai trouvé plus vivant. Enfin moi le 2 c'est vrai que je l'ai bien aimé.

D'accord. Vous vous êtes sentie plus impliquée entre guillemets, c'était le plus interactif ?

$>$ Ouais, hum.

D'accord. Et au niveau du contenu c'est ce qui vous servira le plus au quotidien vous pensez ?

$>$ Oui, voilà. Au quotidien c'est ce qui me servira le plus oui.

D'accord. Alors maintenant est-ce qu'il y a un thème, un sujet concernant le diabète que vous n'avez pas trouvé et que vous aimeriez trouver dans le futur sur le site ?

$>$ Euh... (Longue hésitation) Comme ça c'est dur comme question mais... Non bah moi après il y a les chapitres qui vont se terminer que j'attends. Moi c'est ces deux-là que j'attends en fait. Parce que l'alimentation c'est quand même ce qu'il y a de plus important pour pouvoir gérer donc ça je pense qu'on est quand même beaucoup à attendre euh... et puis l'exercice physique parce que moi j'en fais donc c'est vrai que du coup moi ça c'est vrai que... J'ai été un petit peu déçue parce que j'attendais justement de finir ça pour les visionner. Je suis restée un peu sur ma faim (rires), c'est vrai que j'étais déçue. Le resucrage pendant le sport c'est vrai que c'est quand même important, on ne sait pas toujours comment faire donc euh bon.

Vous ne pensez à aucun autre thème ?

> Alors la pompe c'est vrai que... Nous on nous a parlé de la pompe quand on était à l'hôpital. On ne sait pas trop comment ça fonctionne alors je ne sais pas si...

Peut-être un chapitre dédié au fonctionnement de la pompe alors ?

$>$ Peut-être oui. Pour savoir si ça nous conviendrait, pour peut-être mieux connaitre aussi donc euh...

D'accord. Alors je vais vous demander sur une échelle de 0 à 10 , quelle est votre satisfaction globale vis-à-vis du site INDIGO : 0 vous n'êtes pas du tout satisfaite, 10 vous êtes très satisfaite ?

$>$ Bah 10. (Rires)

Estimez-vous qu'INDIGO vous ait apporté des éléments qui allaient vous aider dans la gestion de votre diabète au quotidien ?

$>$ Oui je pense que ça va m'aider oui. En tout cas ça m'a interpellée, je sais que c'est des questions que je vais poser à ma diabéto dans la gestion euh..., surtout le ressenti des hypos et des hypers parce que j'ai trouvé que c'était bien.

Les hypos et les hypers et puis vous m'avez dit le carnet INDIGO ?

$>$ Oui, oui.

Vous voyez autre chose ? On avait parlé de l'acétonurie aussi ?

$>$ Ah bah ça oui, oui. Il faut vraiment que je l'imprime. Le seul truc c'est que c'est dommage qu'il y ait pas, alors peut-être que je l'ai pas vu moi, mais dans le sommaire peut-être où sont les documents à imprimer.

Un petit onglet particulier pour les documents que l'on peut imprimer ?

$>$ Oui, voilà « c'est là » parce que là du coup je vais devoir me retaper tous les chapitres. (Rires)

Pour terminer est-ce que vous conseilleriez le site INDIGO à une autre personne diabétique de type $\mathbf{1}$ ?

$>$ Ah oui, oui, je le conseillerais oui. (Rires)

Et à un membre de votre entourage ? Vous avez l'intention de le montrer à d'autres personnes ?

$>$ Je voulais le montrer, oui, à une collègue du travail pour en cas d'utilisation du Glucagon, oui.

Cette séquence en particulier ?

$>$ Oui

Avez-vous autre chose à me dire sur INDIGO ?

$>$ Non, j'ai trouvé, enfin moi je sais, alors perso peut-être parce que ça fait pas très très longtemps qu'on a le diabète, ça nous interpelle beaucoup. Par contre je me demandais si quelqu'un qui avait depuis 20 ans du diabète, il regarderait le site.

D'accord. Donc vous, vous pensez être le « public » idéal pour INDIGO ? Un diabète...

$>$... Je pense que quelqu'un qui a un diabète depuis longtemps peut-être qu'il va retrouver des informations, ça serait dommage qu'il passe à côté alors en se disant peut-être que ça fait 20 ans que je l'ai donc je m'en fous un peu de savoir, je sais comment le gérer.

Merci. 


\section{Entretien n ${ }^{\circ} 11$ :}

Pour commencer, quelle est la première chose que vous aimeriez me dire après avoir exploré le site INDIGO ?

$>$ Alors euh... Bon après je ne sais pas si c'est parce qu'aujourd'hui je travaillais et que du coup j'étais peut-être plus fatiguée ou je ne sais pas, mais je l'ai trouvé très long en fait. Quand je suis allée sur le site j'ai trouvé ça très long. Si on parcourt tout le site bah en fait j'ai trouvé ça, voilà, assez long.

Comme on devait avoir l'entretien aujourd'hui, vous avez regardé les trois chapitres en une fois ?

$>$ Voilà.

Vous pensez que si vous aviez eu un peu plus de temps, vous auriez découpé le visionnage ?

$>$ Voilà je n'aurais pas fait le site en entier d'un coup. Je pense que j'aurais fait peut-être chapitre par chapitre ou euh... parce que c'est vrai que sur la fin ça faisait un petit peu long en fait.

Vous vous êtes ennuyée ou y est-ce qu'il y avait trop d'informations d'un coup ?

$>$ Non, non, non. C'est que... peut-être parce qu'en fait c'est très complet, ce qui est très bien. Mais du coup, voilà, je crois que c'était assez long. Mais ce n'était pas de l'ennui, non.

Vous pensez que l'idéal pour visionner le site, c'est certainement en plusieurs fois, ça serait plus confortable entre guillemets ?

$>$ Peut-être, voilà je pense. Après c'est mon avis quoi.

Est-ce que vous avez d'autres choses à me dire sur le site INDIGO ?

$>$ Euh... non après visuellement parlant, c'est très bien. Enfin après il y a peut-être des vidéos qui euh... après bah moi je suis comédienne euh... de temps en temps, enfin intermittente du spectacle, et les vidéos c'est vraiment... enfin moi ça me faisait rigoler parce que moi je trouvais que c'était très mal joué mais est-ce que c'est ça qu'il faut pour que les gens comprennent bien comment ça se passe ? Je sais pas mais il y a des vidéos je trouvais ça vraiment euh... Ouais, je sais pas.

Vous pouvez me donner un exemple?

$>$ Euh... un exemple... Euh par exemple pour le Glucagon, quand euh... voilà chez le pharmacien... C'est pas un film hein, donc c'est pas... c'est normal que ce soit comme ça.

Est-ce que ça a eu une incidence sur la façon dont vous l'avez regardé et qu'est-ce que ça a provoqué chez vous ? Vous m'avez dit que ça vous avait fait sourire...

$>$ Oui, voilà ça m'a fait plus sourire qu'autre chose, mais au final on regarde quand même. Après peutêtre que j'étais moins sérieuse mais euh... c'est vrai que oui ça m'a fait un petit peu sourire.

D'accord. Mais vous avez quand même regardé jusqu'au bout et vous pensez que vous avez appris des choses?

$>$ Oui, j'ai regardé jusqu'à la fin, ce n'était pas gênant non plus.

Vous auriez une suggestion d'amélioration, mis à part le jeu des acteurs, pour cette séquence ?

$>$ Non, non, non, après c'est très pédagogique enfin je sais pas comment dire. Mais c'est vrai qu'on explique bien, que c'est bien montré, donc après c'est pas... c'est pas gênant, voilà ça fait rire sur le moment et puis voilà.

Autre chose ?

$>$ Euh non, non, après euh... non. Non après je ne saurais pas vous dire là tout de suite euh...

Alors parlez-moi de la façon dont vous avez visionné le site. Donc vous m'avez dit que vous aviez vu les trois chapitres. Dans quel ordre vous les avez visionnés?

$>$ Bah dans l'ordre qui était présenté.

A l'intérieur des chapitres, c'est pareil, vous avez regardé les sous-chapitres dans l'ordre qui était proposé ?

$>$ Oui tout à fait, dans l'ordre que c'était. J'ai tout fait dans l'ordre en fait.

Est-ce qu'il y a des choses que vous avez passées plus vite ?

$>$ Euh... bah après il $\mathrm{y}$ a des endroits où ils posaient des questions et il fallait cocher ou des endroits où il fallait marquer des chiffres ou des choses comme ça. Et ça j'avoue qu'il y a des moments où je ne l'ai pas fait.

Certaines fois vous l'avez fait et certaines fois vous ne l'avez pas fait ? Est-ce que vous étiez pressée ? Vous n'aviez pas le temps?

$>$ Voilà je l'ai regardé en entier mais au bout d'un moment, les questions, il y en a je n'ai pas répondu, notamment aussi pour les hypers et les hypos, quand ils demandent les sensations tout ça. Mais bon, fallait que j'aille travailler après donc est-ce que c'est pour ça que... ? Voilà

Est-ce que vous avez envie de retourner le faire quand vous aurez un peu de temps devant vous ?

> Euh bah en fait comme je vous disais, voilà, ce que je n'ai pas fait c'était surtout... enfin il y en a un ou deux que je n'ai pas fait, mais après non je n'y retournerai pas parce que c'étaient des choses que... par exemple comment on se sent quand on est en hypo, en hyper, enfin on le vit donc du coup on sait ce qu'on ressent voilà. Après est-ce que vous, pour faire avancer le site, il faut vraiment le 
faire ? Mais sinon pour moi ça ne m’apportait rien donc du coup je ne me suis pas forcée à répondre non plus.

Vous pensez que ça ne vous aurait rien apporté ?

$>$ Voilà.

Est-ce que vous l'avez consulté seule ou avec quelqu'un ?

$>$ Toute seule. En tout cas, je me suis mise dans un bureau, j'ai fermé les portes, enfin vraiment vraiment toute seule parce que je n'avais pas envie en fait que d'autres personnes entendent, enfin voilà.

On va parler plus précisément du $1^{\mathrm{er}}$ chapitre «Mes objectifs glycémiques ». Est-ce que vous pouvez me dire en une phrase de quoi traite ce chapitre ?

$>$ Alors ce que j'ai compris et emmagasiné c'est qu'ils expliquent en fait le taux de glycémie qu'il faudrait avoir mais que c'est différent en fonction de chaque personne, suivant l'âge, suivant les efforts qu'on fait, notamment les femmes enceintes ou ceux qui travaillent. Voilà enfin ils expliquent bien les différences et que c'est différent en fonction de chacun.

Qu'est-ce que vous avez apprécié dans ce chapitre, soit au niveau du contenu, soit au niveau de la forme?

$>$ Euh... Bah déjà que c'était différent et que c'était... enfin comment dire ? Qu'en fonction du taux c'était pas forcément pas bien, enfin je sais que moi des fois, je vois avec le Pr BENHAMOU, j'ai des résultats pour moi c'est catastrophique et en fin de compte lui il me dit que c'est pas... enfin que ça va. Il me rassure entre guillemets en me disant que c'est pas... voilà c'est pas la fin du monde. Et voilà ça rassure un petit peu de voir ça.

C'est aussi ce que vous a procuré la vision de ce chapitre ? Ça vous a rassurée ?

$>$ Voilà. En fonction des résultats... voilà et puis qu'on ne peut pas tout le temps gérer... enfin voilà. Et puis le fait qu'il y ait beaucoup d'indices extérieurs qui jouent... Enfin j'ai trouvé que c'était bien.

Est-ce qu'il y a autre chose qui vous a plu dans ce chapitre ?

$>$ Euh... Je réfléchis... Non, enfin, c'est pas que ça m'a pas plu hein (rires) mais bon voilà c'est à peu près tout pour ce chapitre.

Est-ce qu'il y a des choses que vous avez moins aimées ?

$>$ Euh... Bah après c'était un petit peu long, enfin j'ai trouvé que ça se répétait un peu parce qu'il y a un moment ; donc le Pr BENHAMOU explique qu'il faut être entre tant et tant ou tant et tant ; et en fait juste après on répète, il y a à nouveau un schéma où on redit la même chose et j'ai trouvé que c'était pas utile. Soit on met l'un, soit on met l'autre, mais mettre deux séquences où on dit la même chose j'ai trouvé que c'était un peu répétitif.

Parce que ces choses n'étaient pas nouvelles pour vous ?

$>$ Euh bah moi en fait on m'a déjà tout bien expliqué avant donc c'est peut-être ça qui fait que j'y ai prêté moins attention, je ne sais pas. Mais moi on m'avait bien expliqué avant donc ça je le savais déjà donc euh... Voilà mais pour quelqu'un qui ne connait pas c'est très bien expliqué et enfin moi je trouve que c'est très utile.

Est-ce qu'il y a autre chose qui était moins bien ?

$>$ Non, non.

Est-ce que les informations contenues dans le chapitre ont répondu aux questions que vous vous posiez sur vos objectifs glycémiques?

$>$ Après comme je vous ai dit, vu que je les connaissais déjà à peu près, que je les avais déjà définis avec mon diabétologue ça m’a pas... Enfin je sais pas comment vous dire, vu que je le savais déjà... On apprend d'autres choses, enfin pas d'autres choses mais euh... on voit qu'il y a plusieurs objectifs glycémiques donc suivant les personnes comme je vous disais, mais moi ils sont déjà fixés donc du coup c'était pas... je sais pas trop comment vous dire.

Non c'est très clair. Est-ce que vous avez des suggestions d'amélioration concernant ce chapitre ?

$>$ Euh... A part comme je vous ai dit le fait qu'il y ait deux séquences où on dit à peu près la même chose.

En choisir une des deux : les explications du Pr BENHAMOU en vidéo ou le schéma : le compteur de la glycémie. Si vous ne deviez garder qu'une des deux formes selon votre suggestion, vous garderiez laquelle ?

$>$ Le Professeur.

D'accord. Vous avez une autre suggestion?

$>$ Non.

On va passer au $2^{\text {ème }}$ chapitre «Mes hypos, mes hypers ». Je vais peut-être vous en rappeler les quatre sous-chapitres : «Comment savoir où j’en suis au niveau de ma glycémie », «Comment je traite mes 
hypos et mes hypers », « J'ai des problèmes d'hypos sévères » et « Je veux aller plus loin dans l'analyse de l'estimation de ma glycémie ». D'accord ?

$>$ Oui.

Est-ce que vous pouvez me dire en une phrase de quoi traite ce chapitre ?

$>$ Alors... (Rires) Je suis très mauvaise pour résumer. Je dirais que c'est un chapitre qui nous montre comment on peut prévenir d'une hypo ou d'une hyper ; bon ce qui est plus difficile ; mais en parlant des sensations que ce soit physiques ou intellectuelles et après donc il explique comment se resucrer et... voilà.

Donc ce chapitre, vous l'avez vu en entier, dans l'ordre défini ?

$>$ Oui.

Qu'est-ce que vous avez aimé dans ce chapitre, dans le contenu ou dans la forme ?

$>$ Euh... Bah c'est pas que je n'ai pas aimé mais comme je vous disais tout à l'heure, hypos, hypers, vu qu'on le vit nous-même j'ai pas trouvé que c'était euh... Je sais pas, je l'ai regardé en entier mais c'est vrai que c'était pas une séquence qui m'a le plus intéressée. Je l'ai regardé comme ça mais pas...

Si on détaille : «Comment savoir où j'en suis au niveau de ma glycémie », les sensations quand vous êtes en hypo, en hyper, vous avez l'impression de les maitriser, vous savez les reconnaitre ?

$>$ Oui. Mais après c'est peut-être bien pour quelqu'un qui ne connait pas du tout... Je pense que je le montrerais par exemple à mes parents ou à des amis, je pense qu'ils le regarderaient beaucoup plus intéressés parce que c'est vrai qu'ils ne connaissent pas du tout. Mais moi, vu que je sais... enfin ça nous arrive à nous donc euh...

D'accord. Pour l'instant le site vous l'avez vu toute seule. Vous avez déjà envisagé de le montrer à des gens ?

Non, non franchement. Là-dessus, je sais pas si c'est parce que j'accepte pas trop que je suis diabétique ou je sais pas. Après je pourrais leur en parler et ils regardent tous seuls s'ils veulent. Déjà je ne le regarderais pas avec quelqu'un d'autre.

Vous sauriez m'expliquer pourquoi ?

$>$ Je sais pas. Parce qu'en fait je préfère, enfin... pas le garder pour moi, mais je pense que si je le montrais, ça alarmerait plus qu'autre chose mes parents.

Vous pensez que le site pourrait alarmer votre entourage?

Den disons, par exemple le risque de coma si on fait une hypo trop sévère ou des choses comme ça, c'est vrai que c'est des trucs que je leur ai pas forcément dit. Après peut-être qu'eux se sont renseignés de leur côté, je ne sais pas du tout. Mais ouais non j'ai pas envie... enfin je ne supporte pas que l'on s'occupe de moi: «Fais attention, fais ci, fais ça ». Donc je pense que si je leur montrais ça, je pense que... Juste une fois, c'est un exemple tout bête : une amie qui sait que je suis diabétique, donc je lui avais expliqué comme il faut les hypos, les... et du coup en soirée la première fois, elle n'a fait que me demander si ça allait, si j'avais pas chaud... et je supporte pas. C'est pour ça qu'éventuellement je ne montrerais pas le site à mon entourage.

Vous m'avez dit deux choses : « Ça serait bien de le montrer éventuellement à ma famille et mes amis » et puis après « Non je ne pense pas qu'il faudrait le montrer à mes amis ou ma famille »?

$>$ Je me suis peut-être mal expliquée : oui ça serait bien pour leur faire comprendre, mais moi personnellement je ne voudrais pas.

Vous pensez que dans l'absolu le site pourrait renseigner l'entourage et lui apporter des choses ?

$>$ Exactement. Je pense que c'est très bien parce que ça explique de $\mathrm{A}$ à $\mathrm{Z}$ le diabète en fin de compte, donc du coup ça serait très bien. Mais moi personnellement euh...

Vous auriez peur qu'au contraire ils se focalisent sur les choses graves entre guillemets ?

$>$ Les dangers, les conséquences si on ne fait pas comme il faut, voilà. Sinon je pense que ça serait très bien pour quelqu'un d'autre.

Est-ce que vous pensez qu'il y a certaines choses qui peuvent rassurer au contraire l'entourage dans le site?

> C'est bien parce qu'ils expliquent que voilà si on fait une hypo sévère on peut quand même injecter le Glucagon, enfin après que ça se gère enfin, qu'on peut les prévenir donc du coup on évite de faire un coma parce qu'on le prévient. Donc peut-être que ça rassure oui.

On en était au chapitre «Mes hypos, mes hypers ». «Comment je traite mes hypers et mes hypos» vous n'avez pas non plus appris de choses ? C'est quelque chose que vous maitrisiez ?

$>$ Non.

«J'ai des problèmes d'hypos sévères », vous avez trouvé la séquence un peu sur jouée. Est-ce qu'il vous a apporté quelque chose ce chapitre ?

$>$ Non, ça pareil, je le savais déjà. Je l'ai regardé mais ça ne m'a pas appris... 
Et « Je veux aller plus loin dans l'analyse de l'estimation de ma glycémie », vous savez, c'est le carnet INDIGO et la grille d'analyse des erreurs, vous en avez pensé quoi ?

$>$ Pareil, la vidéo elle m'a fait un petit peu sourire aussi (rires) mais c'est quand même le reflet de la réalité.

Le jeune qui jette son carnet ?

$>$ Oui, voilà.

Et l'utilisation du carnet INDIGO ou la grille d'analyse des erreurs, c'est quelque chose qui vous intéresse, que vous pensez utiliser ou pas?

$>$ Je pense que ça serait bien, honnêtement. Mais comme il le dit dans la vidéo, ça prend beaucoup de temps et c'est super contraignant, d'écrire à chaque fois, bon après est-ce que c'est parce que j'ai un rythme de vie un petit peu en mode accéléré, je ne sais pas, mais je sais que de moi-même je le ferai pas... je le ferai pas, honnêtement. Mais je pense que c'est très bien par contre.

Vous avez trouvé que c'était bien expliqué, que c'était clair ?

$>$ Oui.

Donc vous n'avez rien trouvé de particulier qui vous ait plu dans ce chapitre, à part peut-être ces notions, le carnet INDIGO et la grille d'analyse des erreurs, mais que vous n'utiliserez pas personnellement?

$>$ Voilà.

Est-ce qu'il y a des choses qui vous ont moins plu ? Vous m'avez dit peut-être le côté un petit peu sur joué de « J'ai des problèmes d'hypos sévères », vous pensez à autre chose ?

$>$ Non, sinon non. C'était très bien.

Est-ce que les informations contenues dans ce chapitre ont répondu aux questions que vous vous posiez sur vos hypos et vos hypers?

$>$ Non, comme je vous ai dit, vu que je le savais déjà, ça ne m’a pas apporté grand-chose.

Est-ce que vous avez des suggestions d'amélioration concernant ce chapitre ?

$>$ Non, je trouve que c'était très bien expliqué.

Vous avez été diagnostiquée en quelle année ?

$>$ C'était il y a deux ans je crois à peu près, deux ou trois ans.

Vous avez déjà eu des stages d'éducation thérapeutique ?

$>$ Bah en fait c'est le Pr BENHAMOU qui m'a tout expliqué comme il faut et puis après j'ai été hospitalisée aux Ecrins pendant... je crois que ça avait duré une semaine à peu près, je sais plus.

Ça c'était au moment du diagnostic ?

$>$ Voilà et en fait ils m'ont tout expliqué, on m'a tout très bien expliqué. Et après j'avais fait une journée pour l'alimentation. Et en fait pareil, ils expliquaient tout.

Vous me dites beaucoup que c'est bien, c'est complet etc. Si vous, vous ne pensez pas que vous êtes le meilleur public entre guillemets pour le site, à qui faudrait-il le proposer en particulier ?

$>$ Aux personnes je pense qui viennent juste d'être diagnostiquées, qui viennent juste de voir qu'ils sont diabétiques. Je trouve que c'est très bien parce que ça explique en fait tout... tout le diabète on va dire.

Vous auriez été contente de trouver ce site-là à ce moment-là ?

$>$ Oui, je pense. Mais vu que j'avais été bien informée par l'équipe médicale, au final... Bon voilà. Mais je pense que c'est bien parce que ça explique bien, voilà. Et après peut-être, bon pas pour moi parce que voilà, mais à l'entourage, la famille, les amis, je pense que ça rassurerait les parents... enfin voilà.

D'accord. On va passer au $3^{\text {ème }}$ chapitre «Mes insulines». Je vous en rappelle les 4 sous-chapitres : «Comprendre mon traitement par insuline », « Mes injections d'insuline », « Comment définir mes doses d'insuline » et « Le manque d'insuline : hyperglycémie et acétone ». Donc vous l'avez regardé en entier et dans l'ordre défini ?

$>$ Oui.

Est-ce que vous pouvez me dire en une phrase de quoi traite ce chapitre ?

$>$ Alors il explique en fait à quoi sert l'insuline et comment elle agit et après comment... pas comment l'utiliser mais... savoir les doses qu'il faut s'injecter.

Qu'est-ce qui vous a plu dans ce chapitre au niveau du contenu et de la forme ?

$>$ Les schémas j'ai trouvé que c'était très bien.

Vous pourriez me dire plus précisément ?

$>$ Quand ils expliquent que par exemple l'insuline lente elle dure $24 \mathrm{~h}$ parce que l'on produit quand même du sucre même quand on ne mange pas et l'insuline rapide pareil, les pics à chaque repas, parce que je suis très visuelle en fait donc du coup je trouve que les schémas c'était très bien.

D'accord, vous les avez trouvés informatifs et bien réalisés ?

$>$ Oui, voilà. 
Autre chose ?

$>$ Euh, non, non. Je... c'est tout. Enfin... ouais.

D'accord. Est-ce qu'il y a des choses qui vont ont déplu dans ce chapitre ?

$>$ Euh non. Là tout de suite maintenant, non.

Est-ce que les informations contenues dans le chapitre ont répondu aux questions que vous vous posiez sur vos insulines?

$>$ Bah en fait pareil vu que je le savais déjà ; enfin que... bah pareil le Pr BENHAMOU m'avait déjà expliqué pareil à l'aide de schémas ; je le savais déjà plus ou moins. Après euh... quand ils ont parlé de la pompe, peut-être pendant une fraction de seconde je me suis dit : "Ça serait peut-être bien que j'ai une pompe ». Mais c'est tout.

D'accord. Donc à un moment donné ça vous a fait vous poser des questions sur votre traitement. Vous aviez déjà eu des informations sur la pompe?

$>$ On avait parlé de comment ça marchait, comment ça fonctionnait mais là, actuellement je ne veux pas de pompe. Pendant une fraction de seconde en fait je me suis dit «effectivement ça serait pas mal parce qu'on n'a pas à se piquer tout le temps, on a juste à faire des bolus », mais au final je ne me vois pas me balader avec une pompe en fait, donc c'est pour ça que ça n'est pas quelque chose que je ferai.

Est-ce que vous avez trouvé que les informations sur la pompe étaient complètes et répondaient aux questions que vous vous posiez sur la pompe ou est-ce que vous auriez aimé que l'on développe plus ?

$>$ Bah voilà j'allais vous dire en fait j'ai pas trouvé que c'était très complet mais euh après c'est peutêtre normal, je sais pas, mais effectivement on aurait peut-être pu, pour quelqu'un qui veut mettre une pompe, peut-être s'étendre un peu plus sur le sujet.

D'accord. Donc ça pourrait être une suggestion d'amélioration, plus d'informations concernant la pompe ?

$>$ Voilà.

Alors on va reprendre les trois chapitres et vous allez me dire quel est le chapitre ou le sous chapitre qui vous a semblé le moins intéressant ou répondant le moins à des événements de votre vie quotidienne.

$>$ Comme je vous disais, peut-être le moins intéressant c'était voilà les hypos et les hypers parce que c'est quelque chose que vraiment, enfin que je sais déjà et pas que je gère mais... enfin quand je vais avoir une hypo je le sais, enfin je le sens, je le sais. Mais après c'est quand même dans la vie quotidienne, donc je ne sais pas si... ouais, non, enfin c'est vrai que c'était peut-être le moins intéressant j'ai trouvé. Enfin pour moi personnellement.

D'accord. Au contraire quel est celui qui vous a le plus intéressée ou qui vous semble le plus utile pour gérer votre diabète au quotidien ?

$>$ Euh... (Rires) Je ne saurais pas trop dire, je suis désolée, je ne suis pas d'une grande aide. Euh... Peut-être oui le plus intéressant, ce que je vous disais, l'insuline.

Donc les schémas, les délais d'action ?

$>$ Oui voilà, quand ils expliquent bien qu'elle agit au bout de tant de temps...

Est-ce qu'il y a un thème qui n'est pas du tout abordé dans le site INDIGO et que vous auriez aimé trouvé ou que vous aimeriez trouver dans le futur?

$>$ Après je sais pas si c'est faisable ou si c'est judicieux mais j'aurais peut-être dit la recherche. Comment ça avance, le diabète, le traitement. Je vais dire une connerie mais par exemple la greffe de pancréas, c'est vraiment des bêtises que je dis.

Non pas du tout.

Voilà peut-être la recherche. Bon ça se guérira sûrement pas, je sais pas, mais voilà.

Quel est l'état actuel de la recherche ?

$>$ Voilà, c'est sur ce côté-là.

D'accord. Vous pensez à autre chose ?

$>$ Non, sinon c'est très bien. Je n'ai pas vu les autres chapitres après, mais sinon je trouve que ça retrace bien l'ensemble du diabète et que ça explique vraiment bien ce qu'il en est.

Ente 0 et 10, 0 : vous n'êtes pas du tout satisfaite et 10 : vous êtes très satisfaite, quelle est votre satisfaction globale vis-à-vis d'INDIGO ?

$>\quad$ Moi j'aurais dit 9 .

D'accord. Alors qu'est-ce qu'il vous manquerait pour aller jusqu'à 10 ?

$>$ Après c'est juste voilà, c'est un côté critique, c'est juste les vidéos qui sont très... enfin voilà je trouve que ça ne fait pas très sérieux, mais bon ça explique bien, donc d'un côté c'est ce qu'il faut mais...

D'accord. Donc ça ce sont les scènes qui sont mises en scène justement. Pour ce qui est des témoignages, est-ce que vous les avez appréciés ? 
$>$ Oui j'ai trouvé que c'était bien parce que du coup ça montre un peu d'autres personnes, enfin j'ai jamais rencontré d'autres personnes diabétiques en fait. Du coup j'ai jamais vraiment pu parler avec d'autres personnes de ce qu'elles vivaient, de comment elles le ressentaient. Bon c'est un peu gnangnan hein, mais j'ai trouvé que justement les témoignages, c'était bien de ce côté-là.

Est-ce que vous avez pu vous identifier facilement à ces personnes ?

$>$ Bah forcément quand il y a avait les jeunes, parce que c'est à peu près les même soucis, les mêmes préoccupations... voilà.

Très bien. Est-ce que vous estimez qu'INDIGO vous ait apporté des éléments qui allaient vous aider dans la gestion de votre diabète au quotidien ?

$>$ Honnêtement, comme je vous ai dit, vu que c'est des choses que je savais déjà, honnêtement, non.

Pour terminer, est-ce que vous conseilleriez le site INDIGO à une autre personne diabétique de type 1 ?

$>$ Totalement.

Donc à un membre de votre entourage ? Vous personnellement vous m'avez dit non, mais vous pensez que le site peut s'adresser à l'entourage des patients.

$>$ Voilà. Moi après c'est parce que peut-être je l'accepte pas comme je vous disais, enfin voilà, mais sinon, je trouve qu'une personne qui est diabétique devrait montrer le site.

Alors c'est peut-être pas une question à laquelle il est facile de répondre, mais est-ce que vous pensez que votre entourage serait en attente de quelque chose comme ça ou serait en tout cas intéressé, le regarderait volontiers?

$>$ Je pense que mes parents, oui. C'est vrai que je ne leur en parle pas, enfin dès que... Là par exemple du coup j'étais dans le bureau et il y a mon papa qui est passé et qui a vu que je regardais ce site-là, il a voulu regarder avec moi, j'ai dit non, non, non.

Il a voulu regarder, il était intéressé ?

$>$ Oui.

S'il vous demande de le regarder tout seul, vous lui direz non ?

$>$ Je sais pas. Enfin non s'il veut y aller il peut regarder, mais c'est vrai que je ne vais pas lui dire : «Bah tiens ça serait bien que tu regardes », mais ça c'est moi, ça c'est personnel.

D'accord, sur sa demande.

$>$ Mais ça serait très bien, je pense, pour les entourages d'une personne diabétique, je pense que c'est très bien.

D'accord. Et puis vous avez vu qu'il a deux chapitres qui sont encore en cours de construction : «Mon alimentation » et «Mes activités physiques ». Est-ce que ce sont des chapitres qui vous intéressent ? Est-ce que vous aimeriez être informée quand ils seront disponibles ?

$>$ Oui j'aimerais bien regarder, par curiosité. Oui, bah oui.

Est-ce que vous avez autre chose à me dire sur INDIGO ?

Merci.

Non c'est tout bon. Je vous ai dit tout ce que... Non, c'est tout bon. 


\section{Entretien n ${ }^{\circ} 12$}

Quelle serait la première chose que vous auriez envie de me dire après avoir exploré le site INDIGO ?

$>$ Euh... bah... En fait euh... moi j'ai eu l'impression que c'était plus un DVD ou un diaporama que vraiment un site Internet, de la façon dont c'est fait quoi, il y a des diapositives qui se succèdent avec des vidéos, des graphiques, des textes. Voilà, c'était ma première impression.

Est-ce que ça vous a gênée ?

$>$ Je ne m'attendais pas à ça disons.

Vous aviez des attentes particulières ? Si oui, lesquelles ?

$>$ Bah c'est pas forcément des attentes mais disons que... j'ai déjà eu fait des recherches pour me documenter, m'informer ; que ce soit sur le diabète ou autre chose ; sur Internet et c'est vrai que la présentation est pas forcément comme ça quoi. Moi c'est vrai que je préfère lire en fait des choses pour aller assez vite et là on a à la fois une voix qui parle, un message écrit, des vidéos. Enfin j'ai trouvé que c'était un petit peu lourd en fait.

Vous auriez préféré trouver le même genre de présentation que vous trouvez d'habitude ? Vous n'avez pas apprécié le fait justement que ce soit différent ?

$>$ Pas forcément. Disons que j'ai trouvé que c'était un petit peu long. Pour trouver une information, il fallait écouter, enfin passer toutes les diapositives quoi. Bon après moi c'est vrai que j'ai quand même une bonne connaissance du diabète. Moi ça fait très longtemps que je suis diabétique.

En quelle année avez-vous été diagnostiquée ?

$>$ En 1986.

Vous avez déjà participé à des stages d'éducation thérapeutique j'imagine ?

$>$ Oui. Au tout début et puis ensuite quand je suis passée sous pompe à insuline aussi, j'ai dû forcément être hospitalisée avec des cours entre guillemets sur le fonctionnement de la pompe.

C'était il y a combien de temps?

$>$ En 2007.

Y a-t-il d'autres choses que vous souhaiteriez me dire spontanément sur le site INDIGO ?

$>$ Après ce que j'ai trouvé intéressant c'est de voir des vidéos. Après je ne sais pas si il s'agissait de gens vraiment diabétiques ou d'acteurs mais disons que ça... enfin que c'est sympa de voir d'autres gens qui peuvent être confrontés aux mêmes choses que ce qu'on vit tous les jours.

Ça vous avez apprécié ?

$>$ Oui. C'est inhabituel pour moi parce que je ne connais pas... très peu d'autres personnes qui ont la même chose que moi.

C'est un point positif. Et qu'est-ce qui vous a fait douter sur le fait que ça puisse être de vrais patients diabétiques?

$>$ (Silence) Je ne sais pas, je me suis posé la question.

Il n'y a pas un élément en particulier qui vous a fait penser ça ? C'est juste que vous vous êtes posé la question de savoir s'il s'agissait vraiment de patient ou d'acteurs qui rapportaient des propos de patients ? Voire même des propos de médecins vous avez pensé ?

$>$ De médecins je ne sais pas. C'était plus le vocabulaire utilisé, la façon de s'exprimer.

Est-ce que vous avez trouvé qu'ils disaient des choses que vous pouvez ressentir ? Vous êtes vous sentie proche d'eux ? Vous avez pu vous identifier ou pas?

$>$ Oui, si, si, complètement.

Tout à l'heure vous me disiez que vous aviez un diabète de longue date entre guillemets. Avez-vous appris des choses grâce au site malgré tout ?

$>$ Euh... Apprises et puis révisées. L'histoire des pics d'action d'insuline, tout ça je n'en avais jamais entendu parler par exemple.

Les délais de...?

$>$... Les délais si mais il y a un moment donné où ils parlent des pics d'action. L'insuline agit dans les 5 à 10 minutes et que c'est là qu'elle est le plus active.

Ça ce n'est pas une notion que vous aviez ?

$>$ Non voilà.

Pouvez-vous me raconter comment vous avez visionné le site ? Vous l'avez consulté seule ?

$>$ Oui.

L'avez-vous consulté en entier ?

$>$ Non pas en entier et pas d'une traite non plus. J'ai l'ai fait en plusieurs fois.

Dites-moi vers quoi vous êtes allée en premier et pourquoi ?

$>$ Euh bah d'abord le premier chapitre, pour voir, par curiosité. Donc là j'ai suivi bien toutes les étapes, toutes les vidéos, les petits questionnaires tout ça. Et après je suis allée me promener dans les chapitres suivants d'autres jours mais de manière plus ponctuelle. 
D'accord donc vous n'avez pas fait par exemple le chapitre $\mathbf{2}$ en entier puis le chapitre $\mathbf{3}$ en entier ? Vous êtes allée voir certaines choses dans certains chapitres?

$>$ Oui voilà, je ne pense pas que j'ai tout vu mais je suis allée voir quelques séquences dans ces chapitres-là oui.

Etes-vous revenue plusieurs fois sur certaines choses ?

$>$ Euh... Non. Réécouter plusieurs fois le même...

... Oui. Aller revoir quelque chose que vous aviez déjà vu ?

$>$ Non, non je n'ai pas eu besoin.

Les choses que vous avez laissées de côté entre guillemets, c'était par manque de temps ? Par manque d'envie ? Parce que le titre vous faisait penser que c'était quelque chose que vous maitrisiez déjà ?

$>$ Oui il y a ça et puis aussi par manque de temps.

On va parler du $1^{\text {er }}$ chapitre «Mes objectifs glycémiques ». Pouvez-vous me dire ce que vous avez apprécié dans ce chapitre aussi bien au niveau du fond que de la forme ?

$>$ Le fond j'ai trouvé que c'était bien, que ça rappelait bien. Avec les films on peut s'identifier aux personnes qui parlent. Après la forme j'ai trouvé que c'était un petit peu long quoi personnellement. C'est vrai que moi comme je vous l'ai dit tout à l'heure je préfère lire une information. Que là je trouvais que c'était un peu redondant d'avoir à la fois la personne qui parle plus le texte plus les documents euh... Voilà je trouvais que c'était un petit peu long.

Quelle serait votre suggestion d'amélioration ?

$>$ Bah peut-être avoir le choix parce que je pense que pour certaines personnes ça peut être bien, des personnes peut-être un peu plus âgées, que ça soit sous cette forme là. Mais après pour des gens comme moi un peu plus jeunes euh... peut-être avoir le choix justement entre avoir toutes les informations ou après avoir seulement une information texte, par exemple.

Vous c'est ce que vous auriez choisi, plutôt le texte ?

$>$ Oui parce que je trouve ça plus rapide de lire dans sa tête. Et puis après pouvoir cliquer si on veut approfondir par une vidéo ou quelque chose comme ça.

Y a-t-il d'autres choses qui vous ont plu dans ce chapitre ?

$>$ (Silence) Alors que je me souvienne... Non, je ne sais plus trop quoi dire.

D'autres choses qui vous ont moins plu ?

$>$ (Silence) Non. Non plus.

D'autres suggestions d'amélioration ?

$>$ Après oui c'était au niveau aussi du débit. Moi j'ai un débit qui est assez bon chez moi et c'était quand même euh... c'était quand même saccadé les vidéos. Il y avait des temps d'attente, des temps de chargement.

Vous l'avez regardé sur quel navigateur ?

$>$ Sur Safari.

Et habituellement vous n'avez pas de problèmes de chargement de vidéos ?

$>$ Bah non.

D'accord. Est-ce que les informations contenues dans ce chapitre ont répondu aux questions que vous vous posiez sur vos objectifs glycémiques?

$>$ Oui. Enfin disons moi je m'en posais pas vraiment parce que je les connais bien. Mais ça... ça conforte ce que je pensais.

D'accord. Alors on va parler du $2^{\text {ème }}$ chapitre « Mes hypos, mes hypers ». Je vais vous en rappeler les 4 sous-chapitres : «Comment savoir où j'en suis au niveau de ma glycémie? », « Comment je traite mes hypers et mes hypos? », «J'ai des problèmes d'hypos sévères» et «Je veux aller plus loin dans l'analyse de l'estimation de ma glycémie ». D'accord ?

$>$ Oui.

Ce chapitre vous m'avez dit l'avoir vu mais pas en entier ?

$>$ Oui, assez rapidement, je ne suis pas allée trop dans le détail ouais. Je ne suis pas allée dans tous les sous-chapitres. Mais c'était plus par faute de temps. Je pense que plus tard j'y retournerai.

D'accord. Pour rechercher des choses en particulier? Pour répondre à des questions précises que vous vous posez ? Ou pour refaire un peu le tour, voir...

.... Non c'est plus pour faire le tour, faire le point sur ce qui est montré, sur ce qui est dit euh...

Qu'avez-vous apprécié dans ce chapitre au niveau du contenu ou de la forme ?

$>$ Oui bah c'est un peu comme le chapitre précédent, d'avoir des vidéos, des explications.

Vous ne vous souvenez de rien en particulier ?

$>$ Non là ça me revient plus.

Y a-t-il des choses que vous avez moins aimées ?

$>$ Non, bah pas plus que ce que je vous ai dit tout à l'heure.

Concernant la forme? 
Voilà.

Les informations contenues dans ce chapitre ont-elles répondu aux questions que vous vous posiez sur les hypos et les hyperglycémies ?

$>$ Oui, oui, tout à fait. J'ai trouvé que c'était plutôt bien fait.

Et complet ?

Oui complet, voilà.

Avez-vous des suggestions d'amélioration concernant ce chapitre ?

$>$ Pas particulièrement, non.

On va passer au $3^{\text {ème }}$ chapitre : «Mes insulines ». Il est composé de sous-chapitres qui sont : «Comprendre mon traitement par insuline », « Mes injections d'insuline », « Comment définir mes doses d'insuline ? » et « Le manque d'insuline : hyperglycémie et acétone ». D'accord ?

$>$ Oui.

Vous l'avez visionné de la même façon que le chapitre 2 ? C'est-à-dire pas dans son intégralité mais seulement certains éléments ?

$>$ Voilà.

Qu'avez-vous apprécié dans ce chapitre ?

$>$ J'ai trouvé aussi que c'était complet euh... Moi c'est vrai que maintenant je suis sous pompe donc je n'ai qu'une seule insuline mais avant j'ai eu aussi des traitements avec une insuline lente et puis aussi de la NPH, j'ai eu ça dans le passé. Donc j'ai trouvé que c'était intéressant de voir les différentes insulines qu'on pouvait utiliser.

Y a t-il des choses qui vous ont moins plu dans ce chapitre ?

$>$ Non, non au contraire. J'ai trouvé que c'était intéressant de parler des pics d'action, tout ça là. Ça je ne connaissais pas. Et j'ai trouvé que c'était bien de montrer des graphiques, c'était assez parlant.

Les graphiques vous ont plu ? Vous ont-ils paru faciles à comprendre ? Aussi bien ceux-là que d'autres que l'on peut trouver sur le site ?

$>$ Oui, oui. Et puis je me souviens aussi je ne savais pas que quand on se pique sur une partie du corps qui est plus chaude ou si on prend un bain après avoir fait l'injection ça augmentait la vitesse d'action de l'insuline. Ça je ne savais pas du tout.

C'est quelque chose que vous avez appris aussi et qui va pourra éventuellement vous servir au quotidien ?

$>$ Oui me servir au quotidien et puis bah mieux comprendre si j'ai une hypoglycémie que je n'arrive pas à expliquer ou des choses comme ça oui.

Y a-t-il des choses qui vous ont moins plu dans ce chapitre 3 ?

$>$ Non, rien de plus.

Avez-vous des suggestions d'amélioration concernant ce chapitre ?

$>$ Non, ma remarque était globale pour l'ensemble de ce que j'ai vu en fait. J'aurais apprécié de pouvoir trouver les informations écrites de manière séparée pour pouvoir aller sur le site de manière plus... plus rapide quoi. Voilà.

Le plan du site ne vous a pas aidée à trouver facilement une information précise ?

$>$ Bah ce qui m'a un petit peu gênée aussi c'est que quand on a fini un sous-chapitre on doit retourner au menu pour activer le chapitre suivant. Ça ne se fait pas de manière automatique si on veut voir tout le chapitre d'un coup.

Le chapitre suivant ou le sous-chapitre suivant ?

$>$ Euh le sous-chapitre suivant. On est obligés de repasser par le menu.

D’accord. Vous auriez préféré que les choses s'enchaînent automatiquement ?

$>$ Voilà oui. Que ça puisse s'enchaîner aussi. Que l'on puisse accéder au menu quand on veut mais que l'on puisse aussi enchaîner sans revenir par le menu.

D’accord. Et concernant le plan général du site qu'en pensez-vous ?

$>$ Je le trouve plutôt logique et bien hiérarchisé.

Si l'on reprend les trois chapitres, quel est le chapitre ou le sous-chapitre qui vous a semblé le moins intéressant ou répondant le moins à des événements de votre vie quotidienne ?

$>$ (Silence) Euh... Le moins intéressant... Bah ce qu'il y a dans le chapitre 2 peut-être.

Les informations sur les hypos et les hypers ?

$>$ Oui parce que c'est quelque chose que je connais tellement bien que ça ne m'a pas plus intéressée que ça parce que je fais souvent quoi.

Au contraire, quel est le chapitre ou le sous-chapitre qui vous a le plus intéressée ou qui vous semble le plus utile pour gérer votre diabète au quotidien ?

$>$ Alors moi ce qui m'a le plus intéressée c'est quand ça parle des insulines, les pics d'action tout ça. Ça peut m'aider aussi à gérer mon diabète et c'est aussi par curiosité d'avoir plus de connaissances sur l'insuline. 
D'accord. Y a-t-il un thème qui n'est pas abordé dans le site INDIGO et que vous aimeriez trouver dans le futur?

$>$ Les chapitres suivants ne sont pas encore terminés hein c'est ça ?

Non effectivement. Les chapitres «Mon alimentation» et «Mes activités physiques» ne sont pas encore disponibles. Souhaitez-vous être informée lorsqu'ils le seront ? Est-ce que ce sont des thèmes qui vous intéressent ?

> Oui, oui ça m'intéresse bien et c'est vrai que dans le chapitre «Mon alimentation » moi ce que j'aimerais trouver c'est des informations sur l'insulinothérapie fonctionnelle.

En dehors de ces deux chapitres qui sont en préparation pensez-vous à d'autres choses qu'il serait intéressant de pouvoir trouver? Des questions que vous vous posez sur le diabète ?

$>$ Moi je m'en suis beaucoup posé ; mais là je ne m'en pose plus ; c'est quand j'étais enceinte de mes enfants. Là je me suis vraiment posé beaucoup de questions sur qu'est-ce qu'il se passait quand on est enceinte, parce que ça perturbe beaucoup le diabète et là je pense qu'on est très en recherche de comprendre, de mieux connaître le diabète dans ce cas-là. Moi j'ai fait beaucoup de recherches sur des forums, des choses comme ça à l'époque.

Avez-vous pu trouver des choses qui vous ont satisfaite ?

$>$ Oui bah sur le site des mamans diabétiques il y a pas mal de choses qui sont bien. C'est intéressant.

Vous pensez que ce serait très intéressant qu'il y ait un chapitre qui soit consacré à la grossesse sur le site INDIGO ?

$>$ Pour des futures mamans diabétiques je pense que ça pourrait être bien. Moi je vais m'arrêter là, j'en ai deux. Moi c'est trop tard. (Rires) Mais je pense que pour d'autres ça pourrait être bien oui.

Sur une échelle de 0 à 10, quelle est votre satisfaction globale sur le site INDIGO : 0 vous n'êtes pas du tout satisfaite, 10 vous êtes très satisfaite ?

$>$ (Silence) 7 .

Alors pouvez-vous me dire pourquoi 7 et pas 8 ou 9 ? Qu'est-ce qu'il vous manque pour mettre une note supérieure ?

$>$ Bah moi j'ai bien aimé le contenu mais la forme m'a un petit peu dérangée quoi.

D'accord done si je reprends ce que vous m'avez dit, dites-moi si c'est juste, vous aimeriez qu'il y ait plus de choses à lire ?

$>$ Oui.

Mais les vidéos de témoignages vous trouvez que c'est quelque chose d'important?

$>$ Oui.

D'accord. Avez-vous d'autres suggestions d'amélioration ?

$>$ Disons que j'ai dû passer beaucoup de temps à passer d'une diapo à l'autre et j'aurais aimé pouvoir avoir autant d'informations mais en y consacrant un peu moins de temps.

Est-ce le fait de devoir cliquer à chaque fois pour passer d'une diapositive à la suivante qui vous a dérangée ?

$>$ Ce n'est pas que ça m'a dérangée mais je ne suis pas habituée quand je vais sur Internet à fonctionner comme ça. J'ai eu plus l'impression d'avoir comme un diaporama ou un DVD.

A qui pensez-vous qu'il soit le plus pertinent de proposer ce site ?

$>$ Bah je pense que c'est plus adapté pour des gens qui sont nouvellement diabétiques, depuis peu, et qui veulent comprendre leur maladie quoi.

Malgré tout est-ce que vous estimez que le site INDIGO vous ait apporté des éléments qui allaient vous aider dans la gestion de votre diabète au quotidien ?

$>$ Oui, oui, je pense. Parce qu'il y a des moments où le diabète on en a un peu jusque là on n'a pas envie de s'en occuper et puis d'autres moments où on a envie de bien faire, on pense à son avenir, à sa santé et puis on veut essayer d'améliorer du mieux possible.

Donc vous pensez que les personnes qui seront les plus enrichies par ce site sont les gens chez qui on vient de découvrir un diabète mais que malgré tout ce n'est pas déraisonnable de le proposer à des gens qui ont un diabète plus ancien ? Qu'ils peuvent y trouver des informations ?

$>$ Oui, tout à fait. Je pense que ça s'adresse à toute personne diabétique en quête d'informations supplémentaires.

Conseilleriez-vous le site INDIGO à une autre personne diabétique de type 1 ?

$>$ Oui, oui, bien sûr.

Conseilleriez-vous le site INDIGO à un membre de votre entourage?

$>$ Pourquoi pas. Si quelqu'un me questionne, oui pourquoi pas.

Pour l'instant ce n'est pas quelque chose que vous avez envisagé ?

$>$ Pas forcément parce que les personnes proches connaissent bien mon problème. Ils n'ont pas forcément de questions par rapport à ça.

D'accord. Merci beaucoup. 


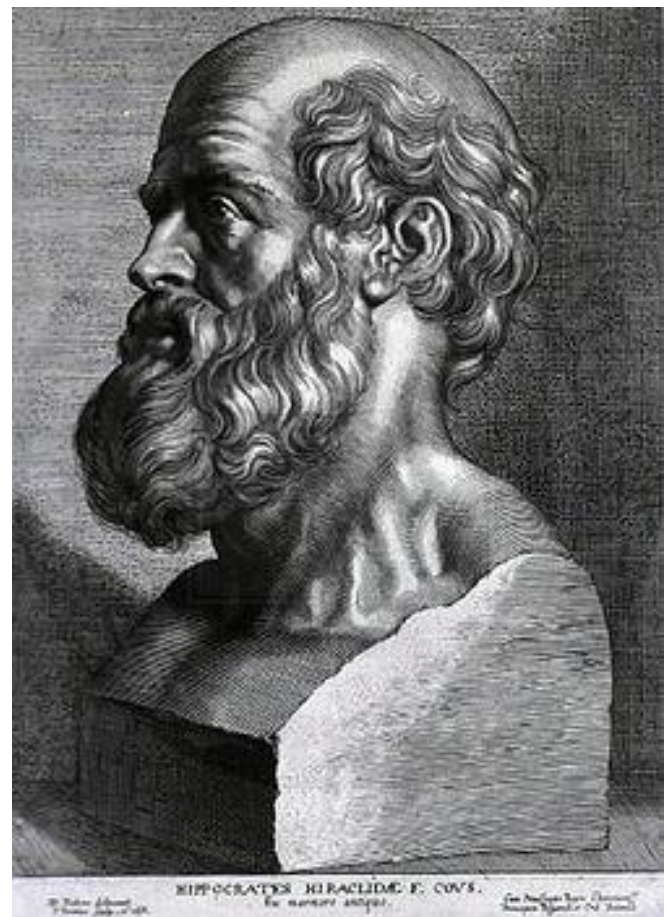

\section{SERMENT D'HIPPOCRATE}

En présence des Maîtres de cette Faculté, de mes chers condisciples et devant l'effigie d'HIPPOCRATE, Je promets et je jure d'être fidèle aux lois de l'honneur et de la probité dans l'exercice de la Médecine. Je donnerais mes soins gratuitement à l'indigent et n'exigerai jamais un salaire au dessus de mon travail. Je ne participerai à aucun partage clandestin d'honoraires.

Admis dans l'intimité des maisons, mes yeux n'y verront pas ce qui s'y passe ; ma langue taira les secrets qui me seront confiés et mon état ne servira pas à corrompre les mœurs, ni à favoriser le crime.

Je ne permettrai pas que des considérations de religion, de nation, de race, de parti ou de classe sociale viennent s'interposer entre mon devoir et mon patient.

Je garderai le respect absolu de la vie humaine.

Même sous la menace, je n'admettrai pas de faire usage de mes connaissances médicales contre les lois de l'humanité.

Respectueux et reconnaissant envers mes Maîtres, je rendrai à leurs enfants l'instruction que j'ai reçue de leurs pères.

Que les hommes m'accordent leur estime si je suis fidèle à mes promesses.

Que je sois couvert d'opprobre et méprisé de mes confrères si j'y manque. 